\title{
Non-invasive imaging of spinal cord blood supply
}

\author{
Citation for published version (APA):
}

Nijenhuis, R. J. (2007). Non-invasive imaging of spinal cord blood supply. [Doctoral Thesis, Maastricht University]. Maastricht University. https://doi.org/10.26481/dis.20070622rn

Document status and date:

Published: 01/01/2007

DOI:

10.26481/dis.20070622rn

Document Version:

Publisher's PDF, also known as Version of record

\section{Please check the document version of this publication:}

- A submitted manuscript is the version of the article upon submission and before peer-review. There can be important differences between the submitted version and the official published version of record.

People interested in the research are advised to contact the author for the final version of the publication, or visit the DOI to the publisher's website.

- The final author version and the galley proof are versions of the publication after peer review.

- The final published version features the final layout of the paper including the volume, issue and page numbers.

Link to publication

\footnotetext{
General rights rights.

- You may freely distribute the URL identifying the publication in the public portal. please follow below link for the End User Agreement:

www.umlib.nl/taverne-license

Take down policy

If you believe that this document breaches copyright please contact us at:

repository@maastrichtuniversity.nl

providing details and we will investigate your claim.
}

Copyright and moral rights for the publications made accessible in the public portal are retained by the authors and/or other copyright owners and it is a condition of accessing publications that users recognise and abide by the legal requirements associated with these

- Users may download and print one copy of any publication from the public portal for the purpose of private study or research.

- You may not further distribute the material or use it for any profit-making activity or commercial gain

If the publication is distributed under the terms of Article $25 \mathrm{fa}$ of the Dutch Copyright Act, indicated by the "Taverne" license above, 
Non-invasive imaging of spinal cord blood supply 
(C) Robbert J. Nijenhuis, Maastricht 2007

All right reserved. No part of this book may be translated or reproduced in any form by photo, photo print, microfilm or any other means without written permission from the author.

ISBN: 978-90-9021965-3

Design and layout: Drukkerij Anraad, Nieuwegein, The Netherlands

Printed by: Drukkerij Anraad, Nieuwegein, The Netherlands 


\title{
Non-invasive imaging of spinal cord blood supply
}

\author{
Proefschrift
}

ter verkrijging van de graad van doctor

aan de Universiteit Maastricht,

op gezag van de Rector Magnificus

Prof. Mr. G.P.M.F. Mols,

volgens het besluit van het College van Decanen,

in het openbaar te verdedigen

op vrijdag 22 juni 2007 om 12:00 uur

door

Robbert J. Nijenhuis

Geboren op 28 februari 1979 te Utrecht 


\section{Promotores:}

Prof. dr. M.J.H.M. Jacobs

Prof. dr. J.M.A. van Engelshoven

\section{Copromotor:}

Dr. ir. W.H. Backes

\section{Beoordelingscommissie:}

Prof. dr. J.T. Wilmink (voorzitter)

Prof. dr. P.E.J.H.M. Kitslaar

Prof. dr. W.H. Mess

Prof. dr. J.A. Reekers (AMC, Amsterdam)

Prof. dr. A.K. Thron (Uniklinikum Aken, Duitsland)

Financial support by the Netherlands Heart Foundation for the publication of this thesis is gratefully acknowledged.

Additional financial support was generously provided by: Cook Medical, Kodak Health Group, Schering Nederland, Toshiba Medical Systems Nederland, Vascutek a Terumo company. 


\section{CONTENTS}

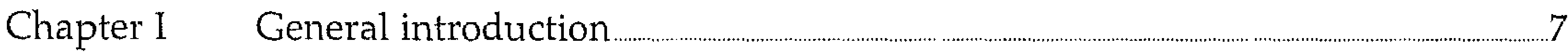

Chapter II Spinal cord feeding arteries at MR angiography for thoracoscopic spinal surgery: feasibility study and implications for surgical approach.

Chapter III Comparison of 0.5-M Gd-DTPA with 1.0-M Gadobutrol for MR angiography of the supplying arteries of the spinal cord in thoracoabdominal aortic aneurysm patients

Chapter IV Differentiation of spinal cord arteries and veins by time-resolved MR angiography

Chapter V MR angiography of the great anterior radiculomedullary artery (Adamkiewicz artery) validated by digital subtraction angiography

Chapter VI MR angiography of the Adamkiewicz artery and anterior radiculomedullary vein: postmortem validation.

Chapter VII Comparison of MR with CT angiography for preoperative localization of the Adamkiewicz artery in thoracoabdominal aortic aneurysm patients.. . .97

Chapter VIII MR angiography and neuromonitoring to assess spinal cord blood supply in thoracic and thoracoabdominal aortic aneurysm surgery.

Chapter IX Value and limitations of MR angiography in spinal arteriovenous malformations and dural arteriovenous fistulas. 133

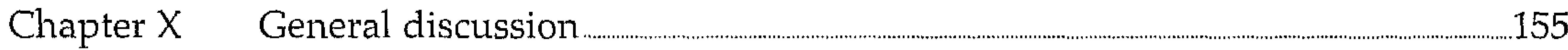

Chapter XI Summary and Conclusions .................................................................................................................... 167

Nederlandse samenvatting …........................................................................................................... 173

Acknowledgements / Dankwoord ……............................................................................................. 181

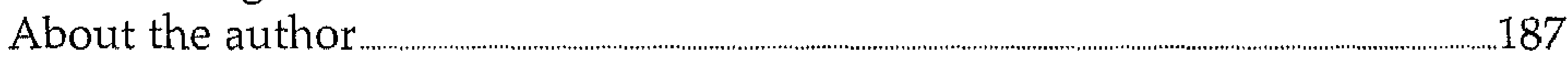

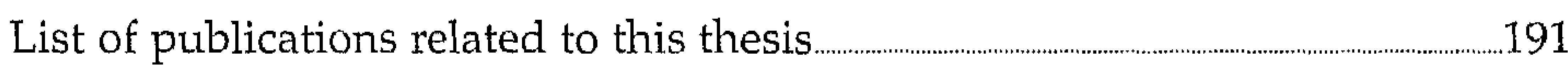




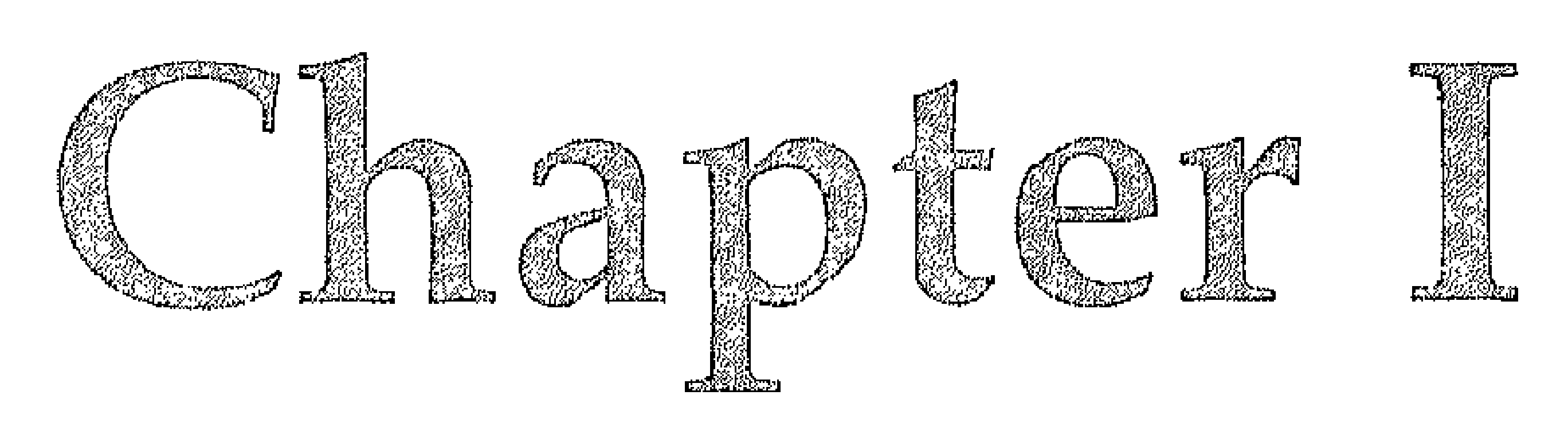

General introduction 
A thoracoabdominal aortic aneurysm (TAAA) is defined as a dilatation of both the thoracic and abdominal aorta. The extent of a TAAA can differ substantially and often presents without symptoms. Therefore, the diagnosis of a TAAA is most frequently based on incidental findings. In patients above 65 years the prevalence of an aortic aneurysm is approximately $5 \% 1$, of which TAAA only comprise $2-5 \% \%^{2}$. Although the prevalence is low, an aortic aneurysms is a potentially fatal disorder as the aorta may rupture. The chance of rupture increases with increasing aortic diameters. When the cross-sectional diameter of the thoracoabdominal aorta exceeds $6 \mathrm{~cm}$, surgery is indicated in which the dilated segment of the aorta is replaced. Unfortunately, TAAA surgery encounters significant complications, the major of which is spinal cord ischemia, that may lead to irreversible spinal cord damage, including paraparesis and paraplegia. The cause of this complication is the interruption of the spinal cord blood supply induced by the aortic cross-clamping during surgery. Intraoperative maintenance of spinal cord function is difficult because the anatomy of the blood supplying trajectories is highly variable and preoperatively unknown in TAAA patients. In this thesis we set out to preoperatively localize the crucial blood supplying trajectories to the spinal cord in order to prevent ischemia during TAAA repair.

Aortic cross-clamping is inevitable when performing open TAAA repair and is considered to be the dominant risk factor for inducing spinal cord ischemia and postoperative neurologic complications. To reduce the potentially harmful effects of aortic crossclamping several perioperative measures are undertaken, which include: cooling the patients' body ${ }^{3}$, cerebrospinal fluid drainage ${ }^{4}$, distal aortic perfusion 5,6 , reattachment of segmental arteries ${ }^{7}$, and neuromonitoring of the spinal cord function using motor evoked potentials $^{8}$. All these measures have been proven to contribute to the protection of spinal cord ischemia. However, they do not provide exact localization of the spinal cord blood supplying arteries to avoid interruption of this crucial supply.

Ideally one would like to visualize the spinal cord blood supplying trajectories prior to surgery in order to plan strategies to preserve spinal cord perfusion or at least to estimate the risk of spinal cord ischemia. To achieve this, spinal cord angiography should depict the blood supplying arteries to the anterior side of the thoracolumbar spinal cord. The anterior horns of this part of the spinal cord namely accommodate the motor neurons, responsible for motor functions of the lower extremities. The largest inlet artery to the thoracolumbar spinal cord, which is therefore considered to be the most important, is the great anterior radiculomedullary artery, also known as the Adamkiewicz artery. The existence of the Adamkiewicz artery and thereby the importance for preoperatively localizing this artery is, however, doubted. A reason for this doubt is that the Adamkiewicz artery could only be identified in a limited number of TAAA patients (detection ratio 43$86 \%)^{9,10}$ using intra-arterial catheter (X-ray) angiography, also known as digital subtraction angiography. The low detection ratio of catheter angiography, which is regarded as the standard of reference for depicting spinal cord arteries, has led to the believe by some that the Adamkiewicz artery does not exist (anymore) in TAAA patients ${ }^{11}$. Catheter angiography may be associated with severe 
complications $(1.2 \%)^{9}$ including stroke, death, and iatrogenic paraparesis or paraplegia. Although the percentage of iatrogenic spinal cord complications is low, it is significant compared to the occurrence of paraplegia due to surgery ( $4 \%$ in our institute) $)^{8}$. Therefore, preoperative localization of the Adamkiewicz artery - at least using catheter angiography - is generally not recommended in the preoperative work-up. Fortunately, Yamada and co-workers in $2000^{12}$ reported for the first time visualization of the Adamkiewicz artery using non-invasive magnetic resonance (MR) angiography. Although the MR angiography image quality was less than for catheter angiography, the results of Yamada et $\mathrm{al}^{12}$ offered new possibilities for preoperative imaging of the blood supply to the thoracolumbar spinal cord.

The Adamkiewicz artery (calibre $<1.0$ $\mathrm{mm})^{13}$ is often the only supplier to the thoracolumbar spinal cord and has a variable location between the fifth thoracic and second lumbar vertebra. For these reasons spinal cord arteriography is very difficult, in particular using non-selective contrast administration in imaging techniques such as computed tomography (CT) and MR angiography. The normal anatomic blood supplying trajectory to the spinal cord runs directly from the aorta through the segmental artery and Adamkiewicz artery to the anterior spinal artery (Figure 1.1). For a detailed description of the spinal cord circulation the reader is referred to textbooks 13,14 .

In TAAA patients many of the aortic orifices of the segmental arteries, possibly also the segmental artery directly connected to the Adamkiewicz artery, may be occluded by atherosclerotic plaques. Therefore, the spinal cord may become dependent on inter- segmental arterial connections or collateral circulation (Figure 1.2). Regarding the drain age of the spinal cord it is important to mention that the great anterior radiculomedullary vein (calibre $0.5-2.0 \mathrm{~mm})^{13}$ can have a very similar spatial (hairpin) configuration compared to the Adamkiewicz artery. Therefore, it is extremely important to separate the inlet artery (i.e., Adamkiewicz artery) from the outlet vein (i.e., great anterior radiculomedullary vein) when performing non-selective contrast administered angiography of the spinal cord. Without clear differentiation between these two spinal cord vessels, most likely the outlet vein instead of the inlet artery will be depicted due to its larger calibre.

In theory, successful non-invasive spinal cord angiography, either by MR or CT, would allow improved preoperative planning in TAAA surgery considering the potential interruption of the spinal cord blood supply by aortic cross-clamping. Preoperative knowledge on the location of the Adamkiewicz artery and its supplying trajectories could guide the positioning of aortic cross-clamps such that interruption of the spinal cord blood supply is avoided. Moreover, in case temporary ischemia is signalled by intraoperative neuromonitoring, selective revascularization of one or more supplying segmental arteries to the Adamkiewicz artery would be possible, to restore the interrupted spinal cord blood supply.

Non-invasive spinal cord angiography can have a much broader field of application than TAAA surgery only. A non-invasive imaging technique that reliably depicts normal spinal cord arteries and veins could also be used to diagnose the presence of vascular spinal cord pathology. Vascular 


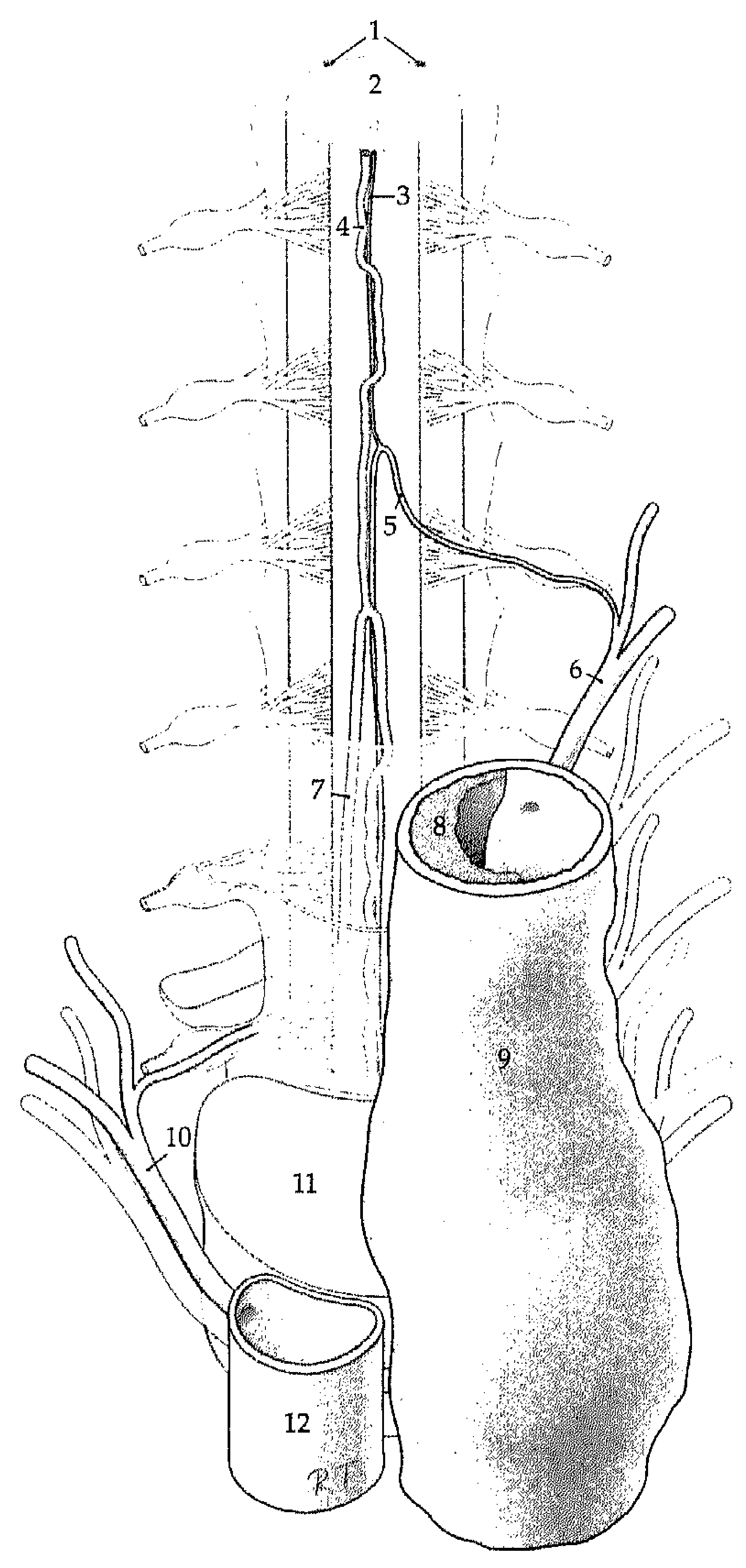

1. Posterior spinal arteries

2. Spinal cord

3. Anterior spinal artery

4. Anterior median vein

5. Great anterior radiculomedullary artery (Adamkiewicz artery)

6. Segmental artery

7. Great anterior radiculomedullary vein

8. Thrombus

9. Aorta

10. Segmental vein

11. Vertebral body

12. Vena cava

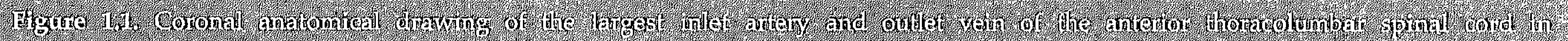

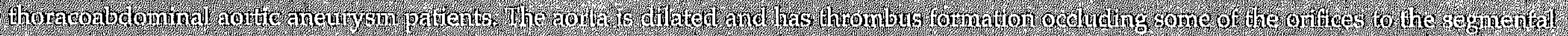

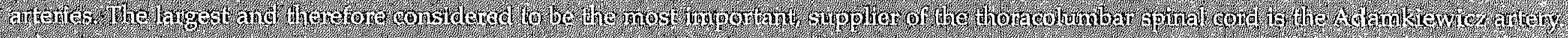

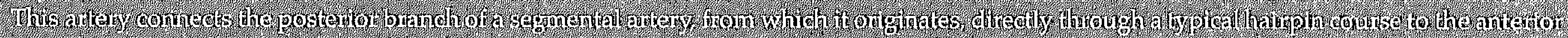

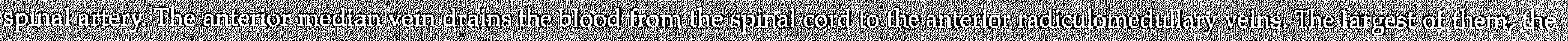

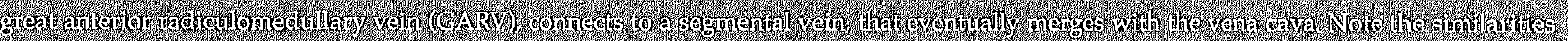

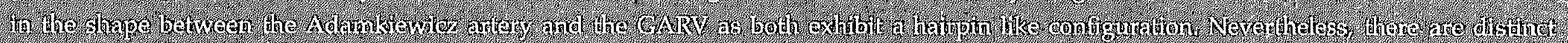

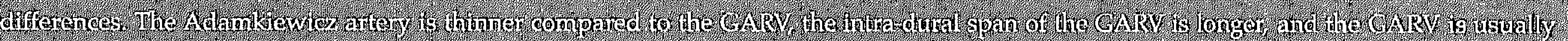

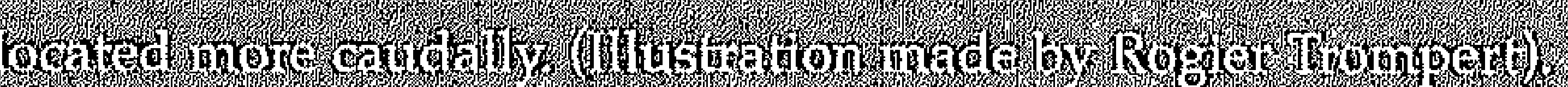




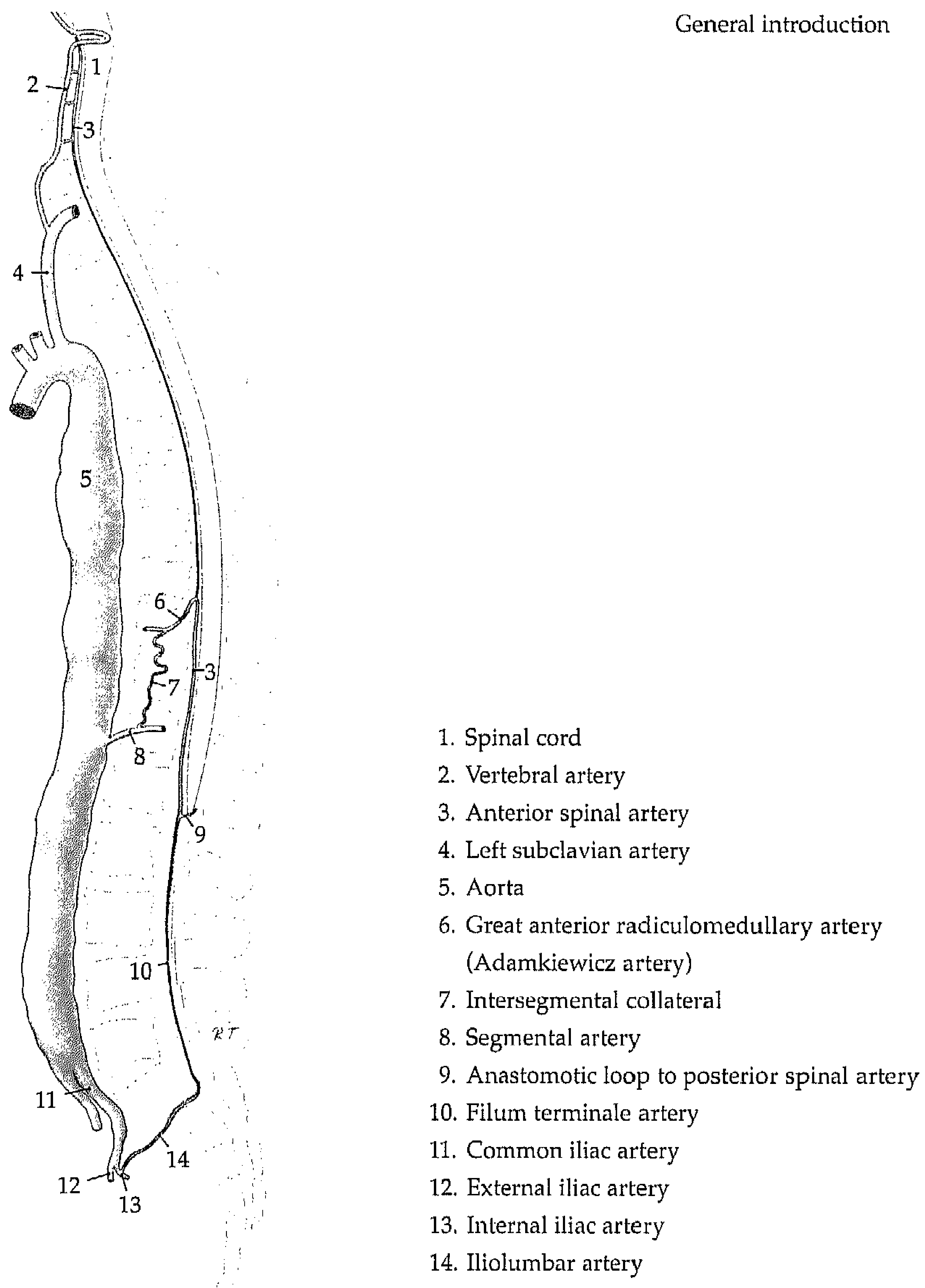

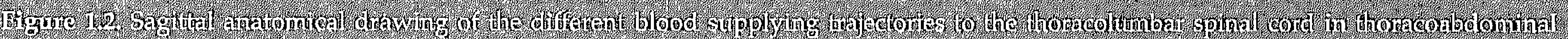

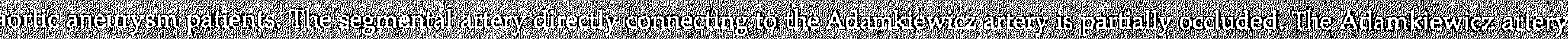

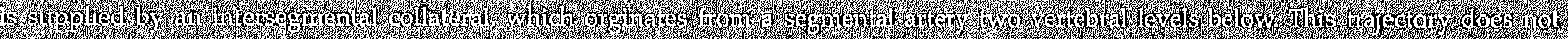

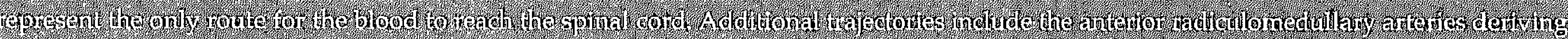

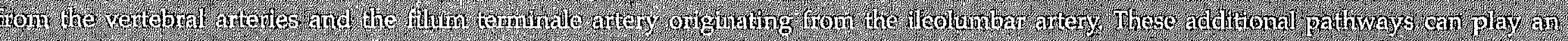

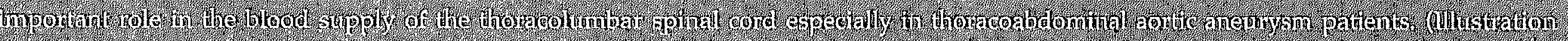

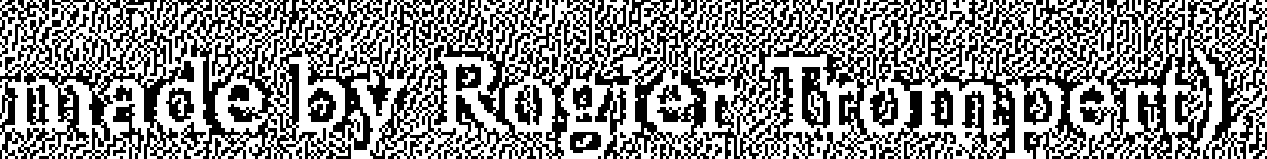


spinal cord abnormalities are usually diagnosed by catheter angiography as this provides the best temporal and spatial resolution to depict the small vessels. However, even in experienced hands localization of the vascular spinal cord abnormality may involve multiple procedures on separate occasions, as the abnormality may be located along the entire vertebral column. Using a non-invasive technique first would allow to focus (and therefore shorten) the subsequent invasive angiographic procedure or even prevent unnecessary (multiple) invasive procedures in case of absence of abnormal vessels. 


\section{Thesis objectives}

1. To develop a MR angiography technique that is capable to reliably detect the blood supply to the spinal cord, in particular the Adamkiewicz artery.

2. To validate the detection of the Adamkiewicz artery as obtained by MR angiography.

3. To determine whether a new contrast agent improves the MR visualization of spinal cord vessels and test for reproducibility of spinal cord MR angiography.

4. To investigate whether CT or MR angiography is the most sensitive imaging technique to localize the Adamkiewicz artery in TAAA patients.

5. To assess whether preoperative detection of the Adamkiewicz artery in combination with intraoperative motor evoked potentials is capable to localize the blood supplying trajectory to the spinal cord that is crucial in TAAA patients.

6. To apply the developed MR angiography technique for the detection of vascular spinal cord abnormalities in patients suspected thereof.

\section{Thesis outline}

- In chapter II, the feasibility of depicting the spinal cord supplying arteries in non-vascular diseased patients with MR angiography, is studied.

- In chapter III, two different contrast agents are applied to optimize and assess the reproducibility of MR angiography in TAAA patients.

- In chapter IV, a novel MR angiography approach based on improved temporal resolution is presented to differentiate spinal cord arteries and veins based on time differences in contrast enhancement.

- In chapter $\mathrm{V}$, the first of two validation studies for the localization of the Adamkiewicz artery is described. This study compared MR angiography with the standard of reference: catheter angiography.

- In chapter VI, the second validation study is presented. A postmortem specimen of a TAAA patient served to validate the location of the Adamkiewicz artery and the great anterior radiculomedullary vein as determined by MR angiography. 
- In chapter VII, a comparative study between CT and MR angiography is described to evaluate which technique is most sensitive to detect the Adamkiewicz artery in TAAA patients.

- In chapter VIII, it is investigated whether it is possible with preoperative MR angiography, in combination with intraoperative neuromonitoring, to determine the blood supplying trajectory, among possibly multiple trajectories, to the spinal cord that is most crucial to preserve spinal cord function.

- In chapter IX, spinal cord MR angiography is applied to detect vascular spinal cord abnormalities prior to catheter angiography.

- In chapter $X$, the findings of this thesis concerning the detection of the Adamkiewicz artery with $C T$ and MR angiography are compared with the existing literature. In addition, the clinical impact of spinal cord MR angiography is discussed and an outlook for future developments is provided.

- In chapter $\mathrm{XI}$, a summary is provided as well as the conclusions answering the aims that were set at the beginning of this thesis. 


\section{REFERENCES}

1. Lederle FA. Ultrasonographic screening for abdominal aortic aneurysms. Ann Intern Med 2003;139:516-22.

2. Cambria R. Management of thoracoabdominal aortic aneurysms. In: Gewertz B, ed. Surgery of the aorta and its branches. first ed. Philadelphia: W.B. Saunders Company; 2000:193-228.

3. von Segesser LK, Marty B, Mueller X, et al. Active cooling during open repair of thoraco-abdominal aortic aneurysms improves outcome. Eur J Cardiothorac Surg 2001;19:411-5; discussion 5-6.

4. Cina CS, Abouzahr L, Arena GO, Lagana A, Devereaux PJ, Farrokhyar F. Cerebrospinal fluid drainage to prevent paraplegia during thoracic and thoracoabdominal aortic aneurysm surgery: a systematic review and meta-analysis. I Vasc Surg 2004;40:36-44.

5. Coselli JS, LeMaire SA. Left heart bypass reduces paraplegia rates after thoracoabdominal aortic aneurysm repair. Ann Thorac Surg 1999;67:1931-4; discussion 53-8.

6. Schepens MA, Vermeulen FE, Morshuis WJ, et al. Impact of left heart bypass on the results of thoracoabdominal aortic aneurysm repair. Ann Thorac Surg 1999;67:1963-7; discussion 79-80.

7. Safi HJ, Miller CC, 3rd, Carr C, Iliopoulos DC, Dorsay DA, Baldwin JC. Importance of intercostal artery reattachment during thoracoabdominal aortic aneurysm repair. J Vasc Surg 1998;27:58-66; discussion -8.

8. Jacobs MJ, Mess W, Mochtar B, Nijenhuis RJ, Statius van Eps RG, Schurink GW. The value of motor evoked potentials in reducing paraplegia during thoracoabdominal aneurysm repair. I Vasc Surg 2006;43:239-46.

9. Kieffer E, Fukui S, Chiras J, Koskas F, Bahnini A, Cormier E. Spinal cord arteriography: a safe adjunct before descending thoracic or thoracoabdominal aortic aneurysmectomy. J Vasc Surg 2002;35:262-8.
10. Williams GM, Roseborough GS, Webb TH, Perler BA, Krosnick T. Preoperative selective intercostal angiography in patients undergoing thoracoabdominal aneurysm repair. J Vasc Surg 2004,39:314-21.

11. Griepp RB, Ergin MA, Galla JD, et al. Looking for the artery of Adamkiewicz: a quest to minimize paraplegia after operations for aneurysms of the descending thoracic and thoracoabdominal aorta. I Thorac Cardiovasc Surg 1996;112:1202-13; discussion 13-5.

12. Yamada N, Takamiya $M$, Kuribayashi S, Okita $Y$, Minatoya K, Tanaka R. MRA of the Adamkiewicz artery: a preoperative study for thoracic aortic aneurysm. J Comput Assist Tomogr 2000;24:362-8.

13. Thron A. Vascular anatomy of the spine. In: Byrne ], ed. Interventional Neuroradiology. Oxford: Oxford University Press; 2002:19-23.

14. Lasjaunias P. Spinal and spinal cord arteries and veins. In: Lasjaunias P, Berenstein, A, Ter Brugge K.G., ed. Surgical Neuroangiography. Berlin: Springer-Verlag; 2001:73-160. 


\section{Chapter II}

Spinal cord feeding arteries at MR angiography for thoracoscopic spinal surgery: feasibility study and implications for surgical approach

RJ Nijenhuis

T Leiner E Cornips JT Wilmink MJ Jacobs

JMA van Engelshoven

WH Backes

Radiology 2004;233:541-7 


\section{ABSTRACT}

Purpose: To prospectively investigate the feasibility of magnetic resonance (MR) angiography for visualization of the spinal vasculature in patients referred for videoassisted thoracoscopic surgical treatment of a thoracic herniated disk and to prospectively assess the influence of preoperative imaging of the spinal vasculature on the choice of surgical approach.

Materials and Methods: Eight patients (three men and five women; mean age, 58 years; range, 42-83 years) with a thoracic herniated disk underwent MR angiography of the thoracoabdominal aorta and posterior branches. Imaging was performed with threedimensional first-pass MR angiography and a triple dose of gadolinium-based contrast agent. Images were analyzed by two observers in consensus to localize the Adamkiewicz artery (AKA) and its connections to the aorta and the anterior spinal artery (ASA). This information was used to determine any change in surgical approach.

Results: In all eight patients, the AKA, the $\mathrm{ASA}$, and the connections with the aorta were identified. The AKA originated between T9 and $L 2$ in all patients and derived from the left side of the aorta in $75 \%$ (six of eight) of the patients. In three patients in whom the AKA was observed on the left side, the surgical approach was changed to the right side to preserve spinal cord integrity.

Conclusions: Preoperative imaging of the AKA is feasible with MR angiography. MR angiography can be used to image the main feeding arteries of the spinal cord in patients undergoing thoracoscopic spinal surgery, and results can be used to change the side of surgical approach. 


\section{INTRODUCTION}

The blood supply of the spinal cord is potentially at risk when a surgical procedure is performed in the vicinity of the spinal cord or the feeding vascular system ${ }^{1,2}$. Operations in which spinal cord blood supply and function are at potential risk are procedures in the thoracic spine ${ }^{1,3}$ such as scoliosis release and correction, fracture treatment, and thoracic discectomy. Although complications of thoracic discectomy are few ${ }^{1-6}$, paraplegia remains possible and is devastating. Therefore, preoperative imaging ${ }^{1-4}$ or spinal cord monitoring with somatosensory evoked potentials 6 has been described and performed for thoracic discectomy.

Change of the side (right vs. left) of surgical approach has been suggested when preoperative imaging reveals that the main feeding artery of the lower spinal cord (T8 through L2) is in the surgical field ${ }^{3,4}$. Nowadays, these surgical procedures are more frequently performed endoscopically with a minimally invasive video-assisted thoracoscopic surgical technique ${ }^{5,7}$. During video-assisted thoracoscopic surgery, segmental arteries are frequently damaged or sacrificed to prevent bleeding8, which could possibly lead to paraparesis or paraplegia. Preoperative imaging of the blood supply system could help in the estimation of the risk for postoperative complications.

The artery that is considered to be primarily responsible for the blood supply to the lower spinal cord is the great anterior radiculomedullary artery, also known as the Adamkiewicz artery (AKA). This artery connects the segmental artery, from which it originates, directly through a typical hairpin configuration to the anterior spinal artery
(ASA). Besides the AKA, which is by definition the largest of possibly many smaller radiculomedullary arteries, more anterior radiculomedullary arteries may arise from segmental arteries at other vertebral levels and also may supply the spinal cord. The vertebral level of the segmental artery, from which the AKA derives, is characterized by substantial interindividual variation. Koshino et $\mathrm{al}^{9}$ showed that the AKA may originate from the vertebral levels of T5 down to L2.

Because of this variability in anatomy of the lower spinal cord blood supply and the potentially devastating consequences of injury to the blood supply during surgery, it is of direct clinical relevance for the patient to undergo preoperative imaging for visualization of the feeding arterial system of the lower spinal cord. The current standard of reference for visualization of the spinal cord vasculature is intra-arterial catheter ( $X$-ray) angiography, also known as digital subtraction angiography, with selective contrast agent injection into segmental arteries ${ }^{10}$. However, catheter angiography involves radiation exposure, it requires injection of potentially nephrotoxic contrast media, and most important, it is not without risk with regard to induction of spinal cord ischemia ${ }^{10}$. Therefore, catheter angiography is not often performed, even though it may yield clinically important information. As an alternative to catheter angiography, magnetic resonance (MR) angiography recently has been described for noninvasive imaging of the spinal vasculature in patients with a thoracoabdominal aneurysm ${ }^{11-}$ 13. The lower degree of detection of the AKA with MR angiography, however, remains an unsolved problem. This could be due to the absence of this vessel in some patients and the use of a suboptimal MR imaging technique. 
Thus, the purpose of our study was to prospectively investigate the feasibility of MR angiography for visualization of the spinal vasculature in patients referred for videoassisted thoracoscopic surgical treatment of thoracic herniated disk and to prospectively assess the influence of preoperative imaging of the spinal vasculature on the choice of surgical approach.

\section{MATERIALS and METHODS}

\section{Phantom study}

To investigate to what limit detection of small-diameter blood vessels is possible with MR angiography, a blood vessel phantom was constructed. The range of blood vessel diameters in the phantom was chosen to correspond to the diameters of the ASA (0.2$0.8 \mathrm{~mm})$ and AKA (0.5-1.2 mm) as known from literature 9,14 . The phantom consisted of ten tubes with varying diameters $(6.00,2.03,1.57$, $1.40,1.01,0.76,0.51,0.25,0.18$, and $0.13 \mathrm{~mm}$ ), and these were filled with a gadolinium-based contrast agent (Magnevist; Schering, Berlin, Germany) with a concentration of $15 \mathrm{mmol} / \mathrm{L}$, which is representative of the arterial concentration ${ }^{15}$. The tubes were immersed in a muscle tissue-mimicking fluid $(0.073 \mathrm{~mol} / \mathrm{L}$ aqueous solution; T1, $800 \mathrm{msec}$ ) and imaged by using the same imaging protocol as was used in the patient study. Acquisition parameters for the T1-weighted gradient-echo sequence were TR $5.9 \mathrm{msec}$; TE $1.9 \mathrm{msec}$; flip angle $30^{\circ}$; and voxel size $0.8 \times 0.8 \times 1.2 \mathrm{~mm}$. Because the relevant arteries have a particular course through the magnetic field, the phantom was imaged separately for two orientations, parallel and perpendicular to the magnetic field.
The ASA, for instance, runs from cranial to caudal direction, which is nearly parallel to the magnetic field. The segmental arteries, on the other hand, run along the vertebrae, perpendicular to the magnetic field. As one looks at the AKA, the first and the last part of this artery are also perpendicularly orientated to the magnetic field. The phantom was slightly (about $5^{\circ}$ ) tilted with respect to the three orthogonal axes of the MR imaging system to allow signal averaging over many subvoxel positions. Signal-to-noise ratio (SNR) was calculated for each tube diameter. For each tube, signal was measured by one observer in a user-defined region of interest that comprised the enhanced parts inside the tubes. These results were used to investigate the relationship between signal level and tube diameter. Regions of interest were drawn after the display of the images was magnified on a graphic workstation (Sun UltraSparc; Sun Microsystems, Sunnyvale, Calif) with commercially available image processing software (Easy Vision, release 4.0; Philips Medical Systems, Best, the Netherlands). All regions of interest had the same length (20 $\mathrm{mm}$ ) along the tube axes; the sizes (i.e., width) of the regions of interest perpendicular to the tube axes increased from 0.1 to $4.0 \mathrm{~mm}$. Background signal and noise were measured and averaged with a region of interest that was placed between the tubes at different positions in the phantom. The distance between the region of interest and the outer tube wall was approximately $1 \mathrm{~mm}$.

\section{Patient study}

From August 2002 until January 2003, eight patients (three men, five women) who subsequently planned to undergo elective surgical repair of a symptomatic thoracic 
herniated disk participated in this study. The mean age was 58 years (range, 42-83 years). None of these patients had any history of vascular disease. The institutional review board of the hospital approved the protocol, and written informed consent was obtained from all patients.

\section{Imaging protocol}

All patients underwent MR imaging preoperatively, and MR imaging consisted of three components. Patients were imaged in the supine position. At first, T2-weighted anatomic survey imaging was performed to localize the vertebrae and the course of the spinal cord. Subsequently, a bolus timing image was obtained to determine the imaging delay. Finally, MR angiography was performed. All acquisitions were performed with a phased-array spine coil and a 1.5-Tesla MR imaging system (Philips Intera, release 8; Philips Medical Systems).

The imaging field of view (FOV) covered the fifth thoracic vertebra (T5) down to the fifth lumbar vertebra (L5) to ensure that the AKA was in the FOV. Anatomic transverse and sagittal T2-weighted survey MR images were obtained. Acquisition parameters were TR $2686 \mathrm{msec}$; TE $120 \mathrm{msec}$; and flip angle $90^{\circ}$. The truly measured voxel size was $1.25 \times 1.67$ x $4.0 \mathrm{~mm}$. When necessary, the study MR imaging was combined with a more elaborate clinical MR imaging, and the combination provided dedicated preoperative information in regard to the herniated disk anatomy.

Synchronization of the sampling of the center of $\mathrm{k}$-space profiles with the peak contrast agent (same as that used in the phantom study) concentration was achieved with injection of a test bolus of $2 \mathrm{~mL}$. From the bolus timing image, the time between the start of contrast agent injection and maximal enhancement of the distal aorta and segmental arteries was determined by using the dynamic viewing mode of the imaging unit. The test bolus was injected at a speed of $3 \mathrm{~mL} / \mathrm{sec}$ with a power-injector (Spectris; Medrad, Indianola, $\mathrm{Pa}$ ), and after injection, a saline flush of $25 \mathrm{~mL}$ was injected at a speed of $3 \mathrm{~mL} / \mathrm{sec}$. The time between the start of injection and total enhancement of the distal aorta was used as the imaging delay in obtaining the subsequent MR angiographic image.

To obtain the MR angiographic image, a triple dose $(0.3 \mathrm{mmol}$ per kilogram body weight) of the contrast agent was injected at a speed of $3 \mathrm{~mL} / \mathrm{sec}$, and after injection, a saline flush of $25 \mathrm{~mL}$ was injected at a speed of $3 \mathrm{~mL} / \mathrm{sec}$. MR angiography was performed with two T1-weighted dynamic phases that each lasted shorter than 40 seconds. This time and the centric k-space filling ensured a difference between the arterial (first phase) and venous (second phase) enhancement. FOV size in the craniocaudal direction (frequency encoding) was $45 \mathrm{~cm}$, and that in the anteroposterior direction (phase encoding) was $16 \mathrm{~cm}$. The number of sections was individually adjusted (range, 70-80) to include the entire vertebral cross section over the total FOV (T5 through L5). The acquisition parameters were as follows: TR $5.9 \mathrm{msec}$; TE $1.9 \mathrm{msec}$; and flip angle $30^{\circ}$. Truly measured voxel size was $0.8 \times 0.8 \times 1.2$ $\mathrm{mm}$. The k-space was filled by using an elliptic centric order (CENTRA; Philips Medical Systems). Acquired sections were $1.2 \mathrm{~mm}$ thick and were reconstructed to sections of $0.6 \mathrm{~mm}$ thickness. 


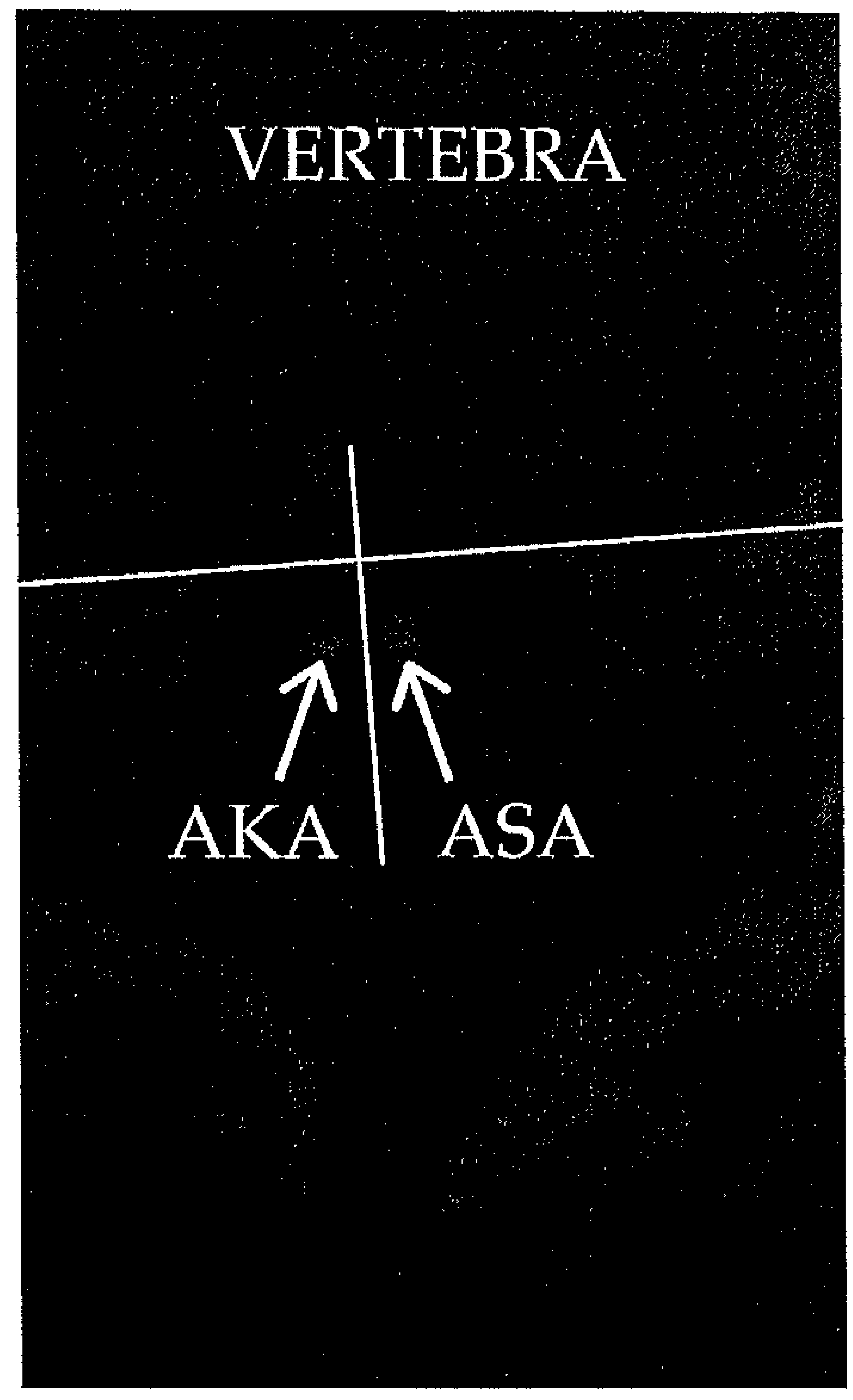

a

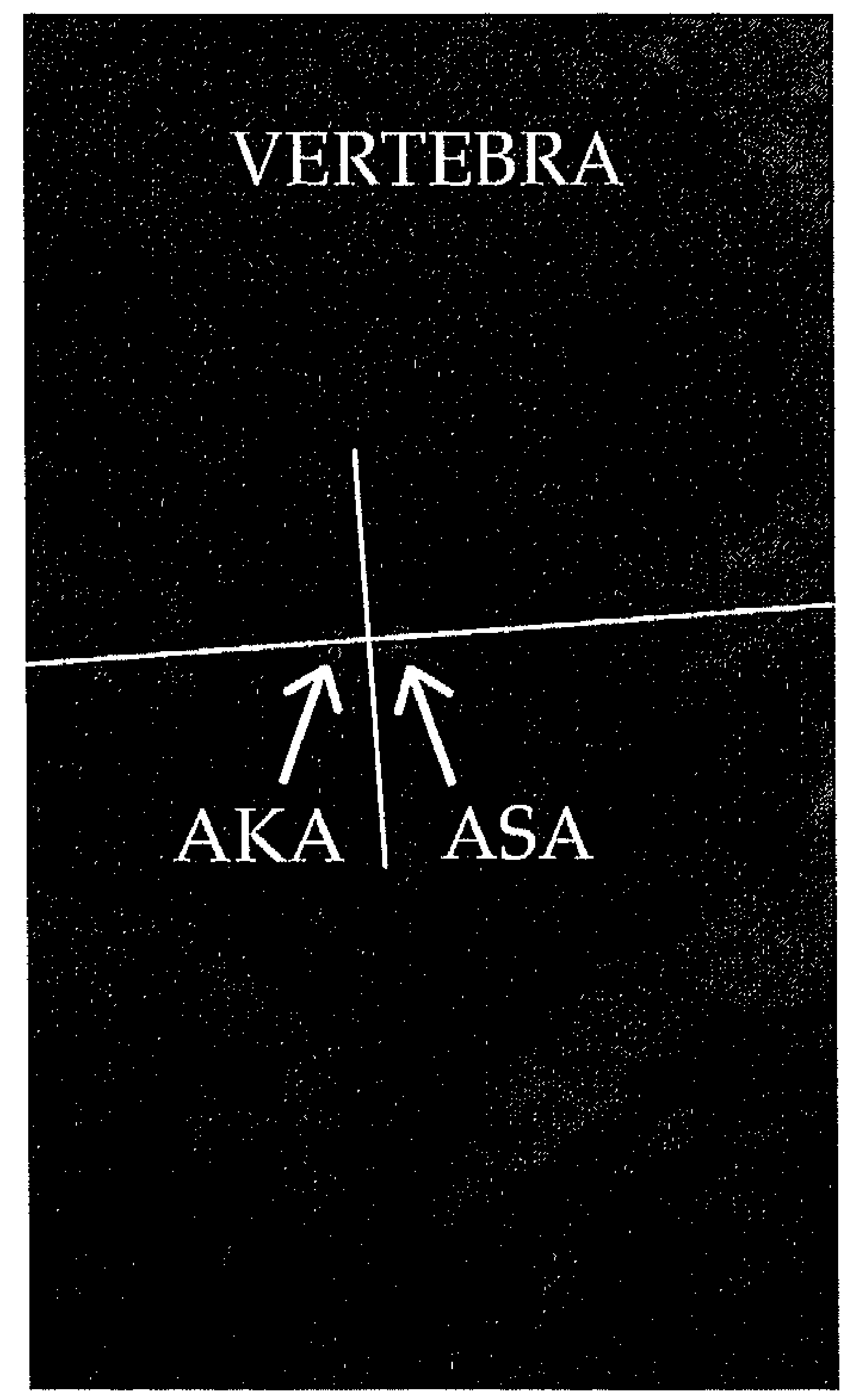

b

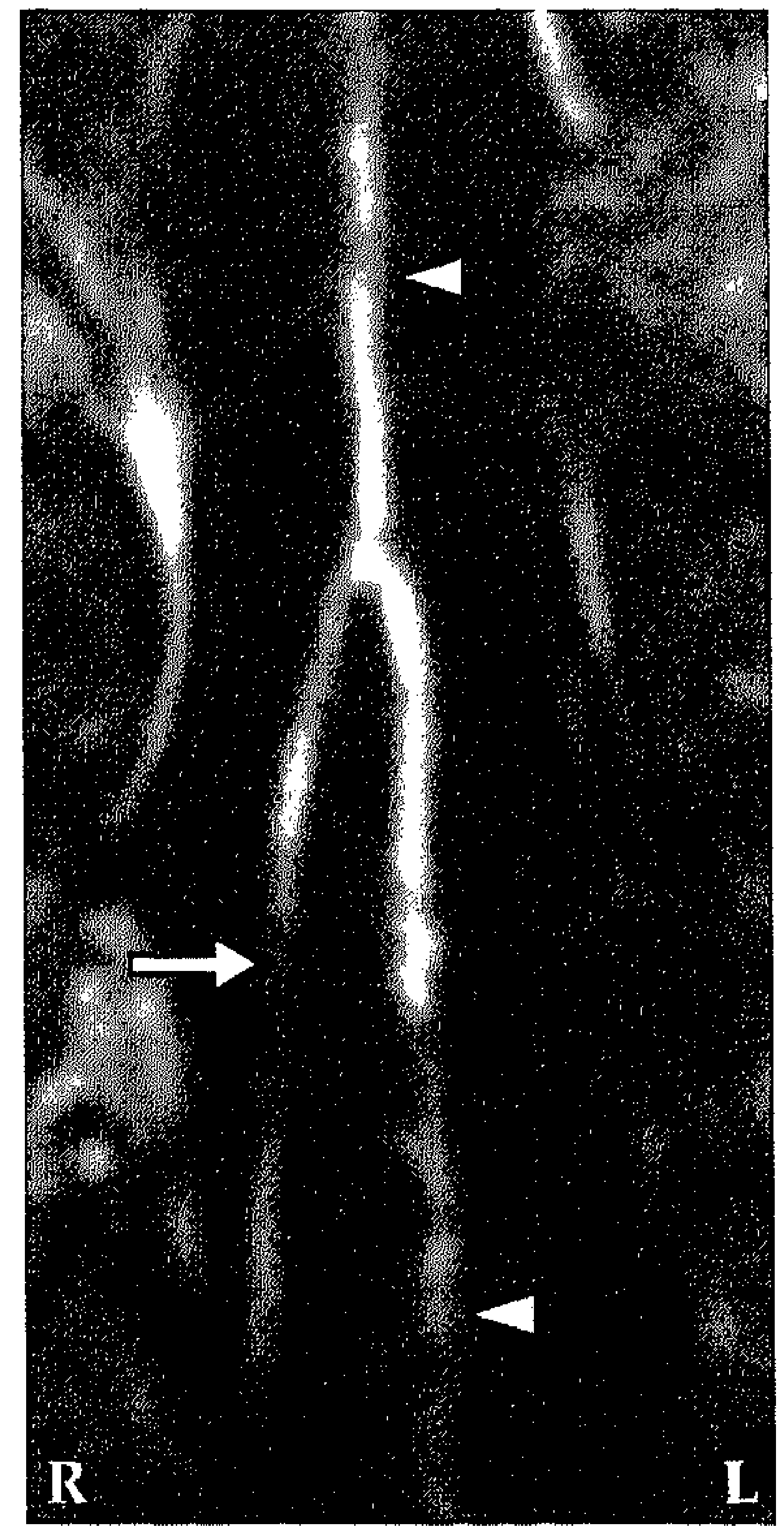

C

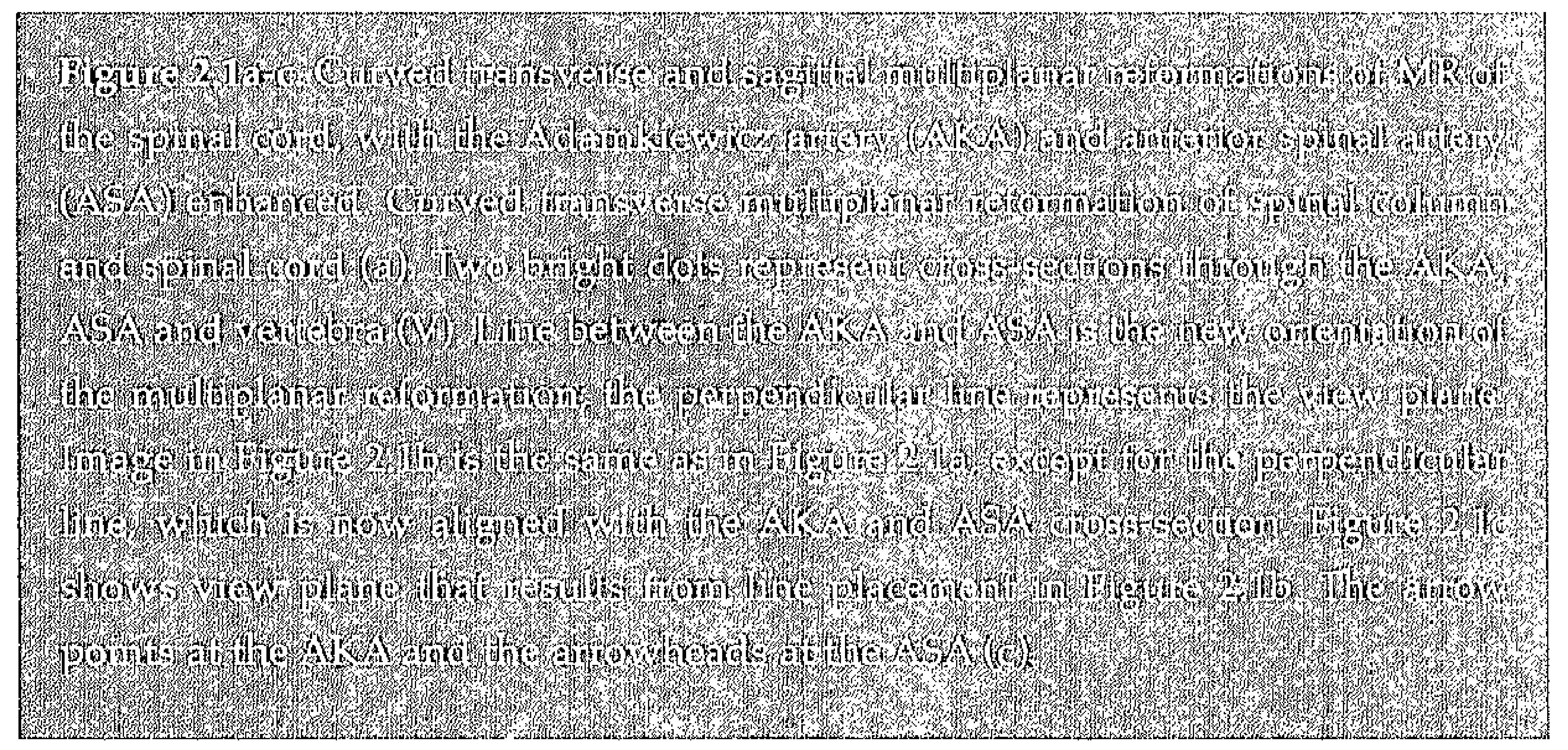

Image analysis

Images in all patients were evaluated in consensus, with consideration of the level and the side of the AKA by two observers (with 2 and 5 years of experience in MR angiography). The MR angiographic images were analyzed by using curved multiplanar reformation and targeted maximum intensity projection of targeted regions at the same graphic workstation and with the same commercially available image processing software as were used for the phantom studies. The transverse and sagittal T2 sections were used for the verification of the vertebral level of the segmental artery that was connected to the AKA. 
The targeted maximum intensity projection was generally used for verification of the segmental artery. With multiplanar reformation, it was possible to search the whole spinal cord for vascular structures starting from L5 and moving upward to T5. With transverse sections, the spinal column was systematically (from vertebra to vertebra) screened upward for the bright appearance of the cross-section through the AKA and ASA. When this cross-section was encountered, a new cross-sectional plane was drawn perpendicular to these two vessels (Figure 2.1), and a new stack of sections was created with multiplanar reformation. When both structures (cross-sections of the ASA and the AKA) were in the same newly created plane, the full length of the AKA became visible.

The following criteria were applied to identify the ASA, the AKA, and the connecting segmental artery: (i) The structures of the vasculature should appear brightest in the first (i.e., arterial) phase (Figure 2.2). (ii) The ASA was identified as an enhanced structure in the middle of the anterior surface of the spinal cord. (iii) An enhanced vessel that ran through the intervertebral foramen toward the spinal cord, with the specific ascending feature, and was connected to the spinal artery at the top of the hairpin curve was considered to be the AKA. (iv) Determination of the vertebral level of origin of the AKA was performed by using the anatomic image as a reference for the vertebral level. The intervertebral level at which the segmental artery entered the spinal cord was defined as the level of origin. For instance, in a case in which the segmental artery entered between T12 and L1, this was considered to be the segmental artery at T12. (v) Only when the enhanced vessel originated between $\mathrm{T} 9$ and
L2 was it considered to be the AKA. If more than one enhanced vessel originated between $\mathrm{T} 9$ and L2, the one with the largest diameter was considered to be the AKA, and those with a smaller diameter were considered to be anterior radiculomedullary arteries.

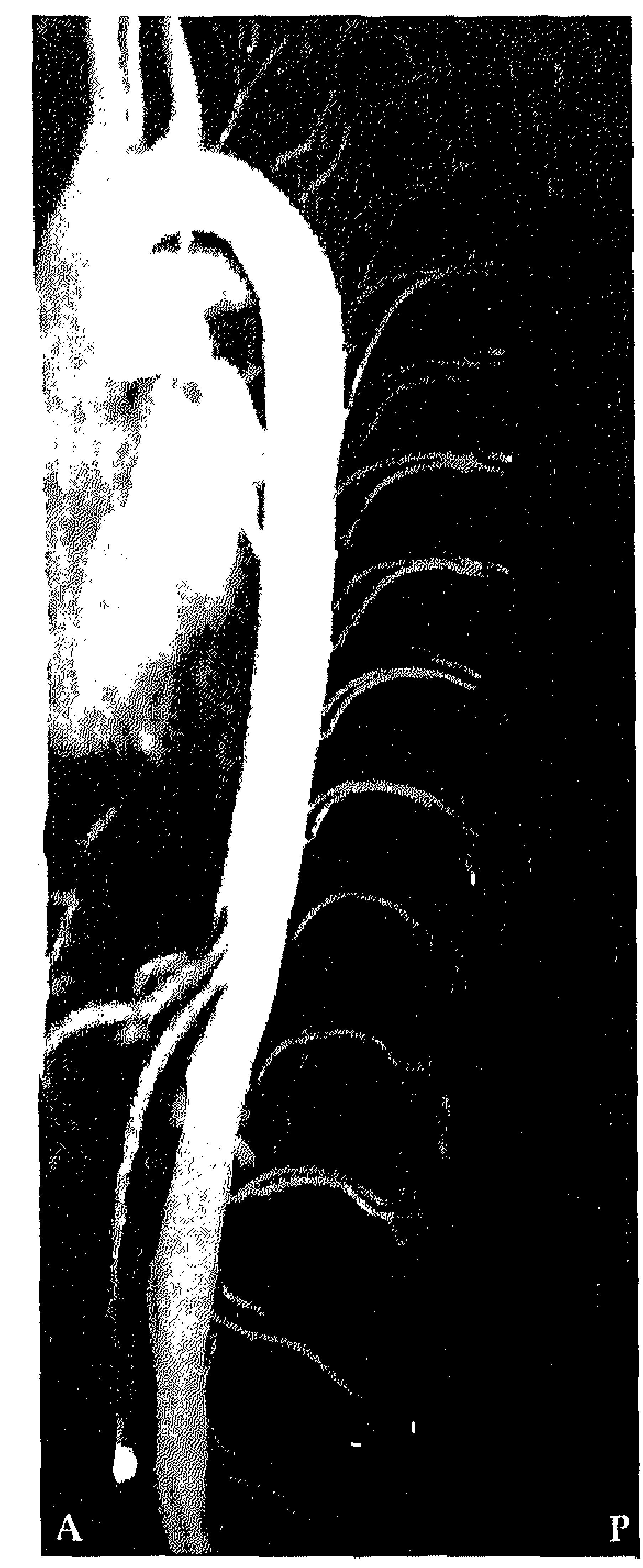

a

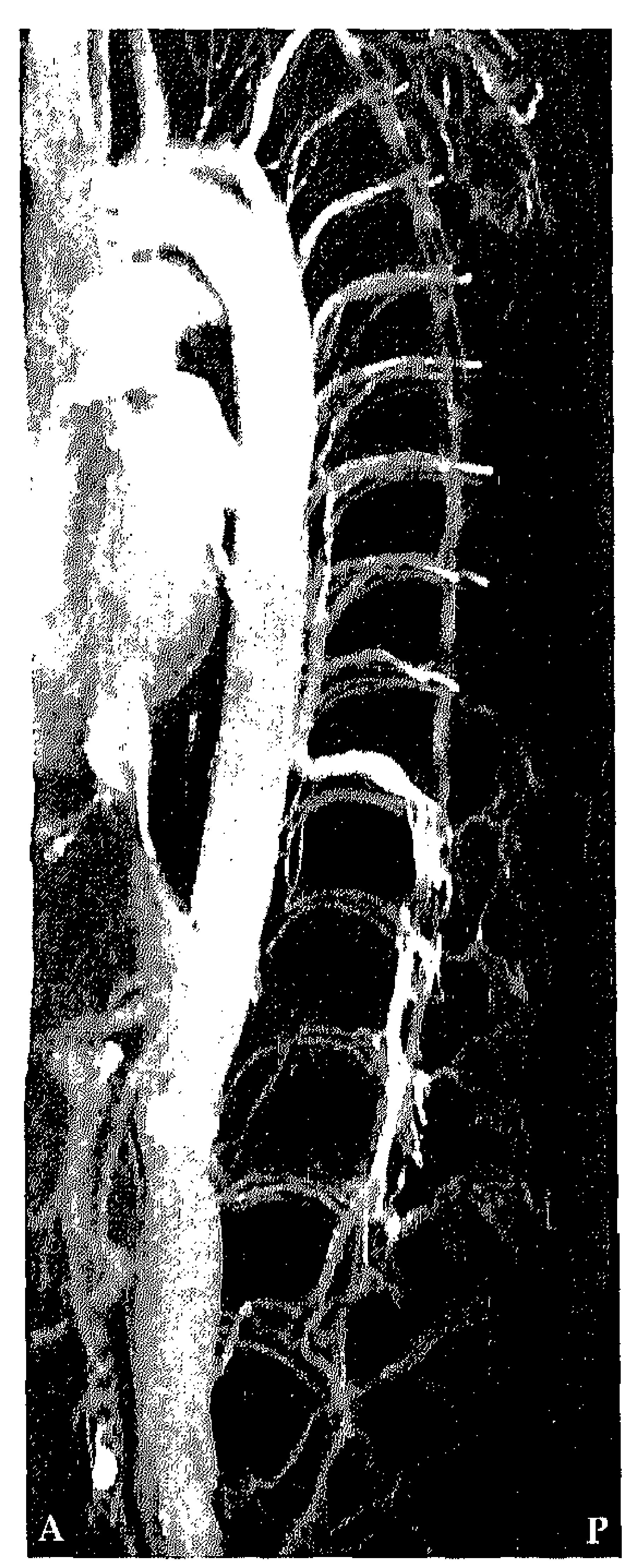

b

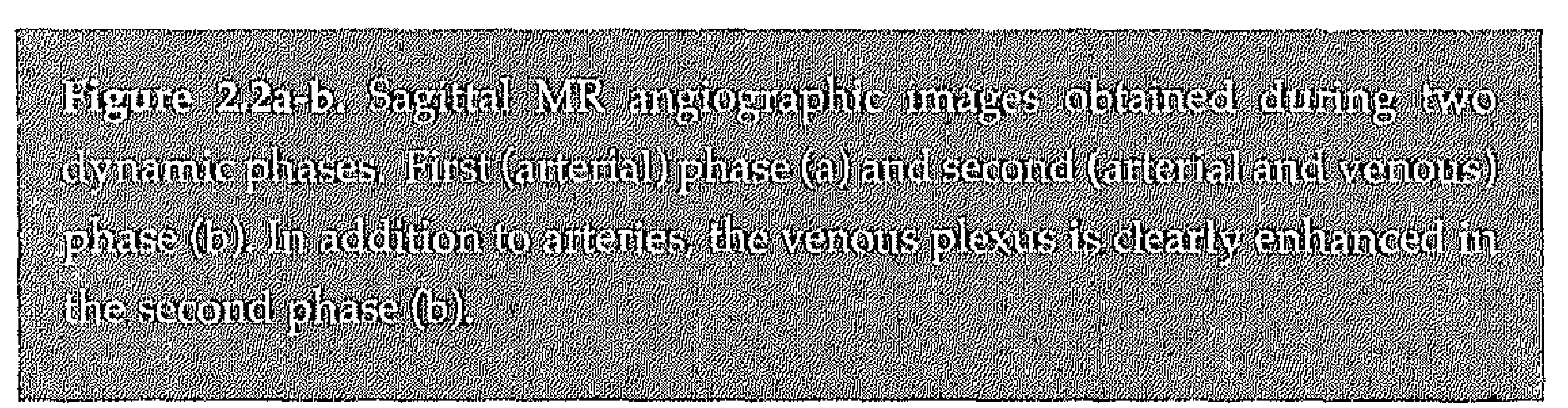




\section{Surgical approach}

Prior to imaging, surgical approach was determined by the neurosurgeon. To investigate whether results of MR angiography influenced the choice of side of surgical approach, the imaging findings were shown to the neurosurgeon, who had 5 years of experience. When the segmental artery that was connected to the AKA was at the same level and on the same side as was the disk herniation, the neurosurgeon was asked if a change of the side of approach from the side originally selected would be needed.

In the postoperative course, patients were monitored by the neurosurgeon for paraplegia and bleeding from segmental arteries, which were considered to be major adverse outcomes.

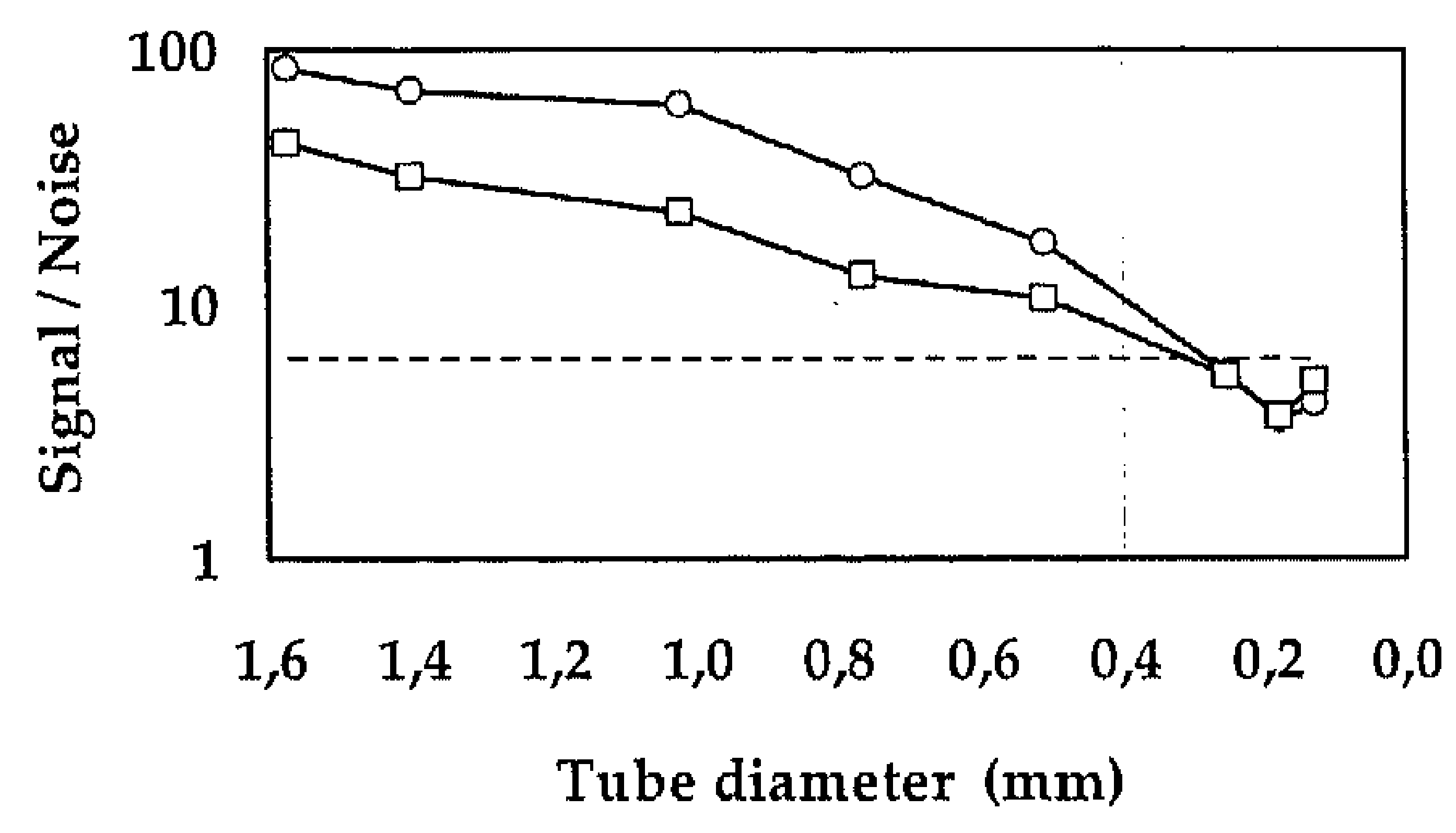

\section{RESULTS}

\section{Phantom study}

For both phantom orientations, the SNR decreased as the tube diameters became smaller (Figure 2.3). The SNR for the perpendicular orientation was decreased overall. A similar course of the SNR as a function of the tube diameter was found for both orientations. At a tube diameter of 0.9 $\mathrm{mm}$, the SNR was decreased by $50 \%$. The

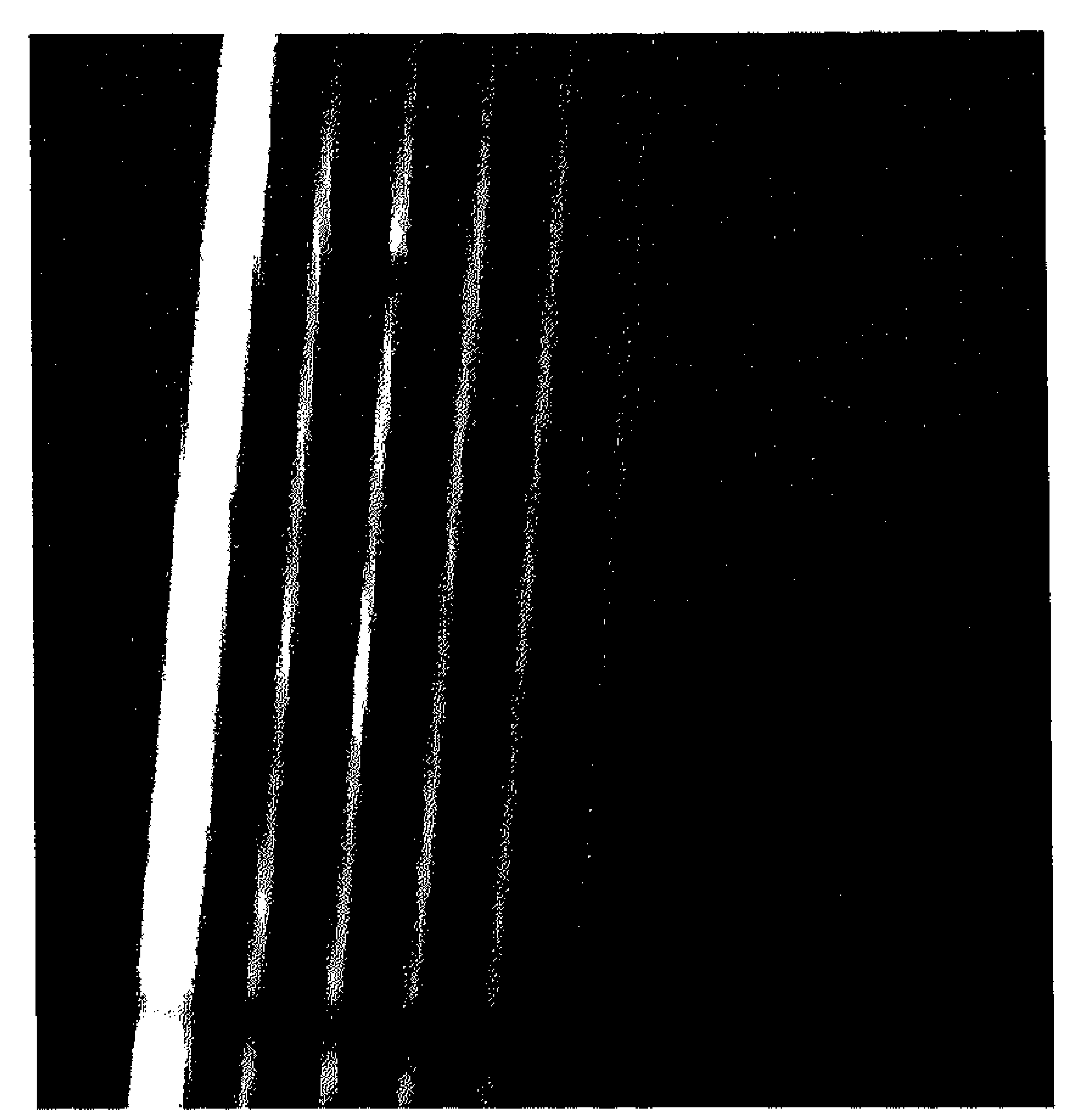

TH

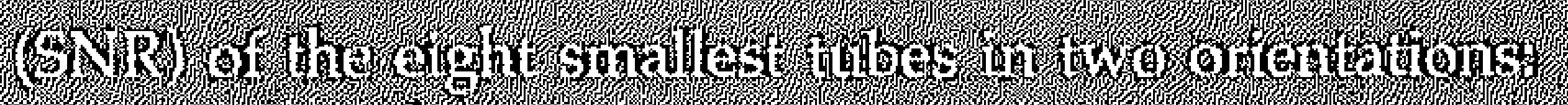

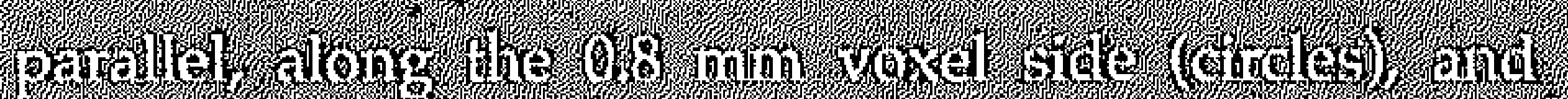

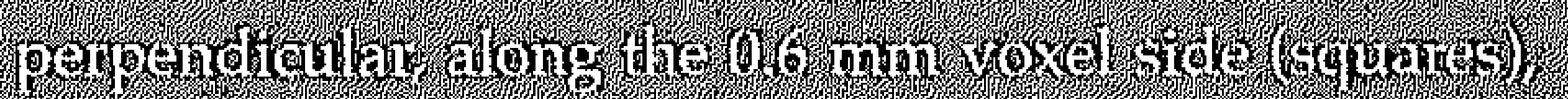

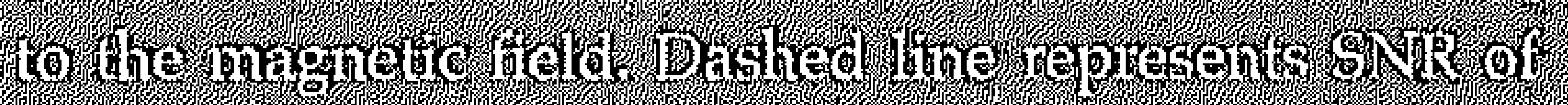

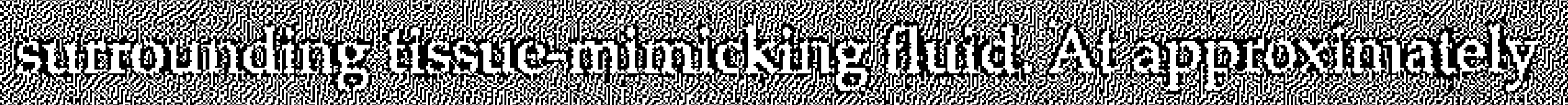

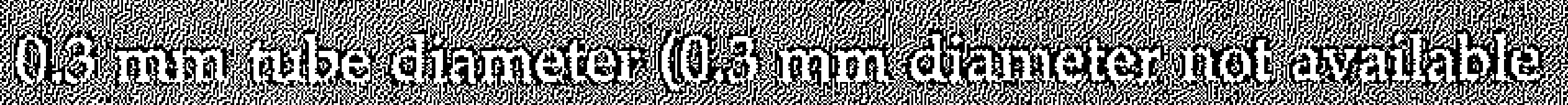

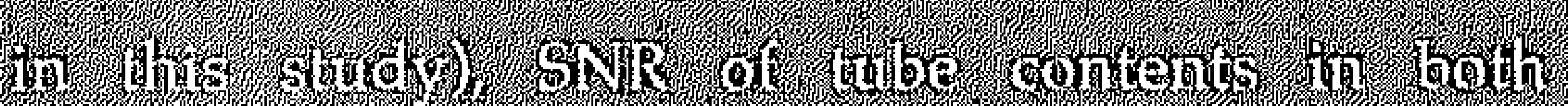

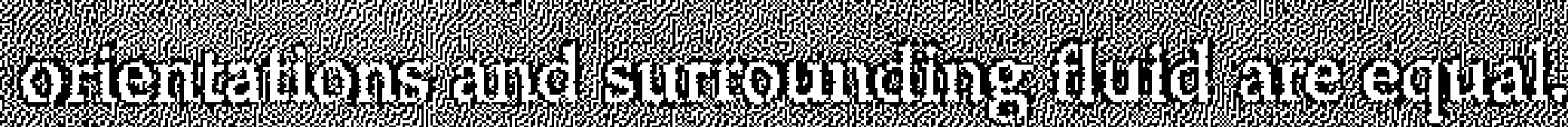

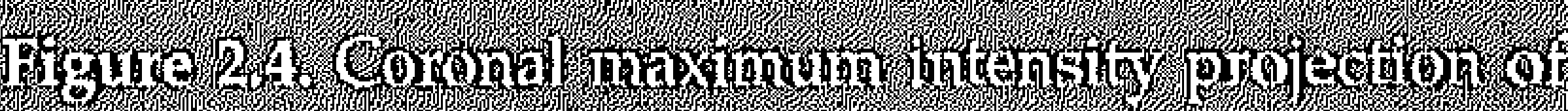

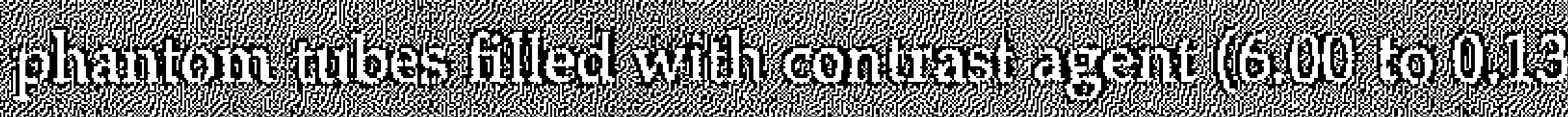

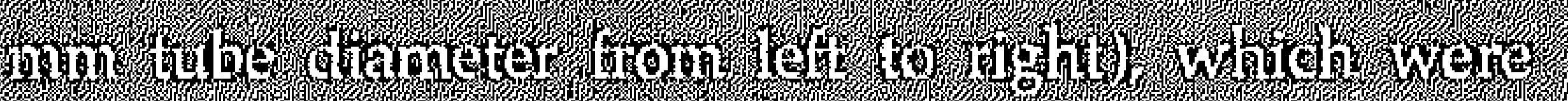

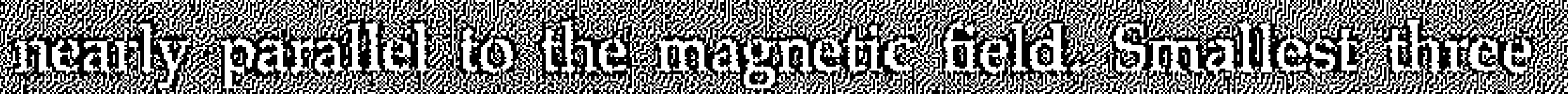

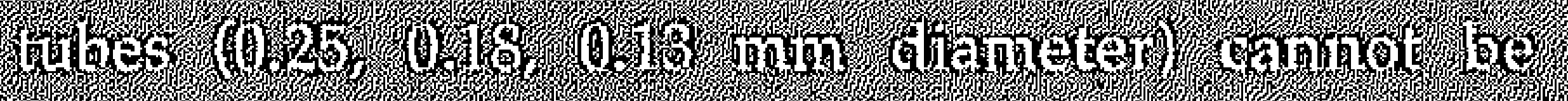

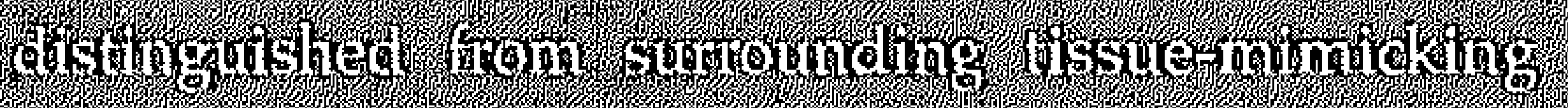

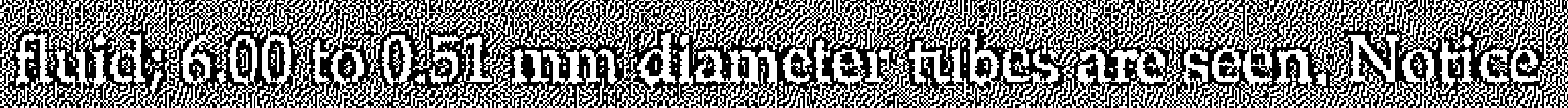

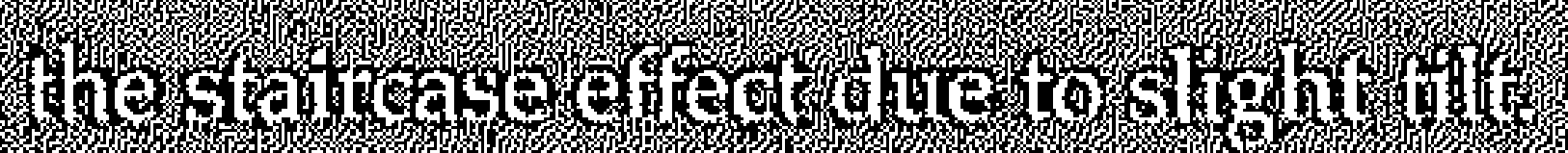


smallest tube diameter, with a more increased SNR than that of the surrounding fluid, was $0.51 \mathrm{~mm}$ (Figure 2.4). At a diameter of approximately $0.3 \mathrm{~mm}$, the SNR of tube contents and that of surrounding fluid were equal. With the smallest diameter $(0.13 \mathrm{~mm})$, the SNR values were slightly increased (Figure 2.3).

\section{Patient study}

In all eight patients, the ASA, the AKA, and the connecting segmental artery were detected (Figure 2.5). Bolus arrival times varied from 5 to 10 seconds among patients. In Table 2.1, the results in our patient group with regard to lateralization and craniocaudal localization of the AKA and ASA are summarized. In six $(75 \%)$ of eight patients, the AKA originated from the left side, and in two patients $(25 \%)$, it originated from the right side. In three cases, more than one anterior radiculomedullary artery was found. The ASA could be followed from T9 down to L2 in six patients. The higher thoracic segment of the ASA (T5 through T9) was not visualized in all cases. In two patients, enhancement of posterior spinal arteries was observed.

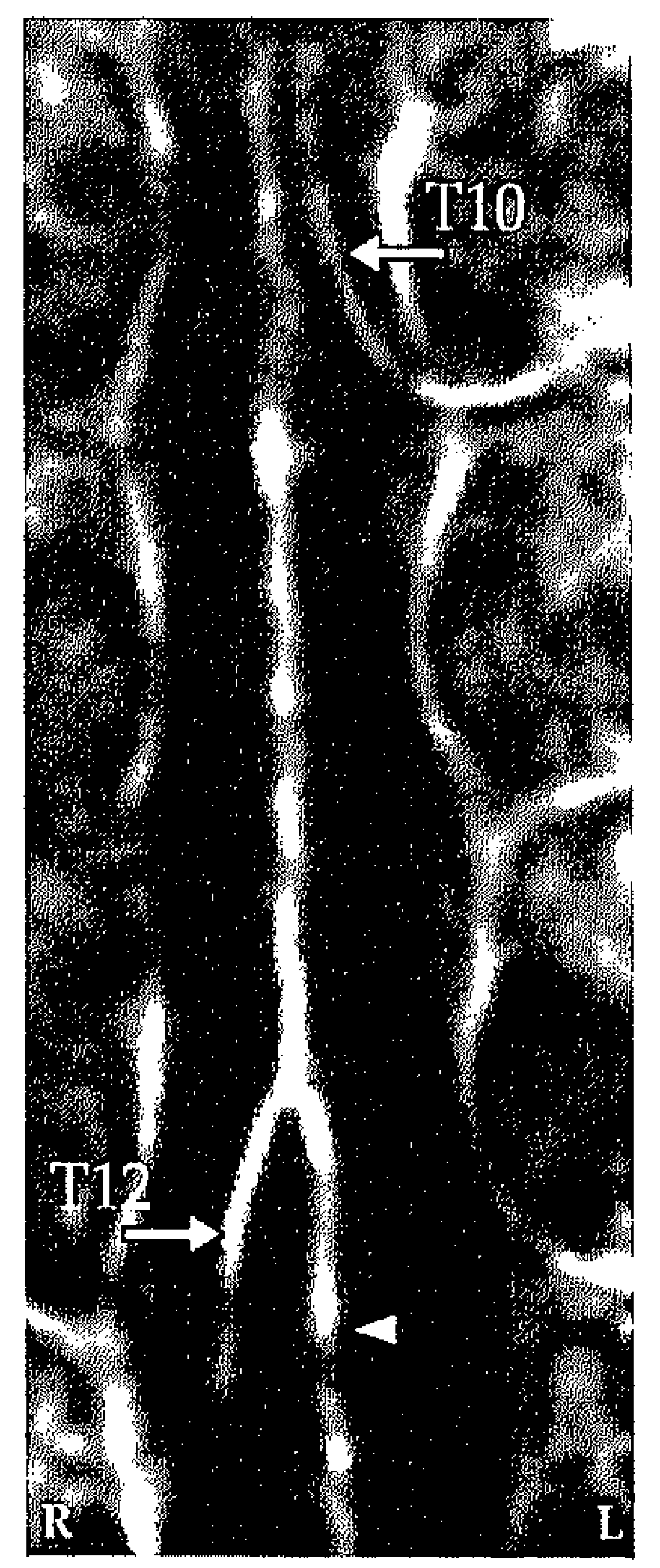

a

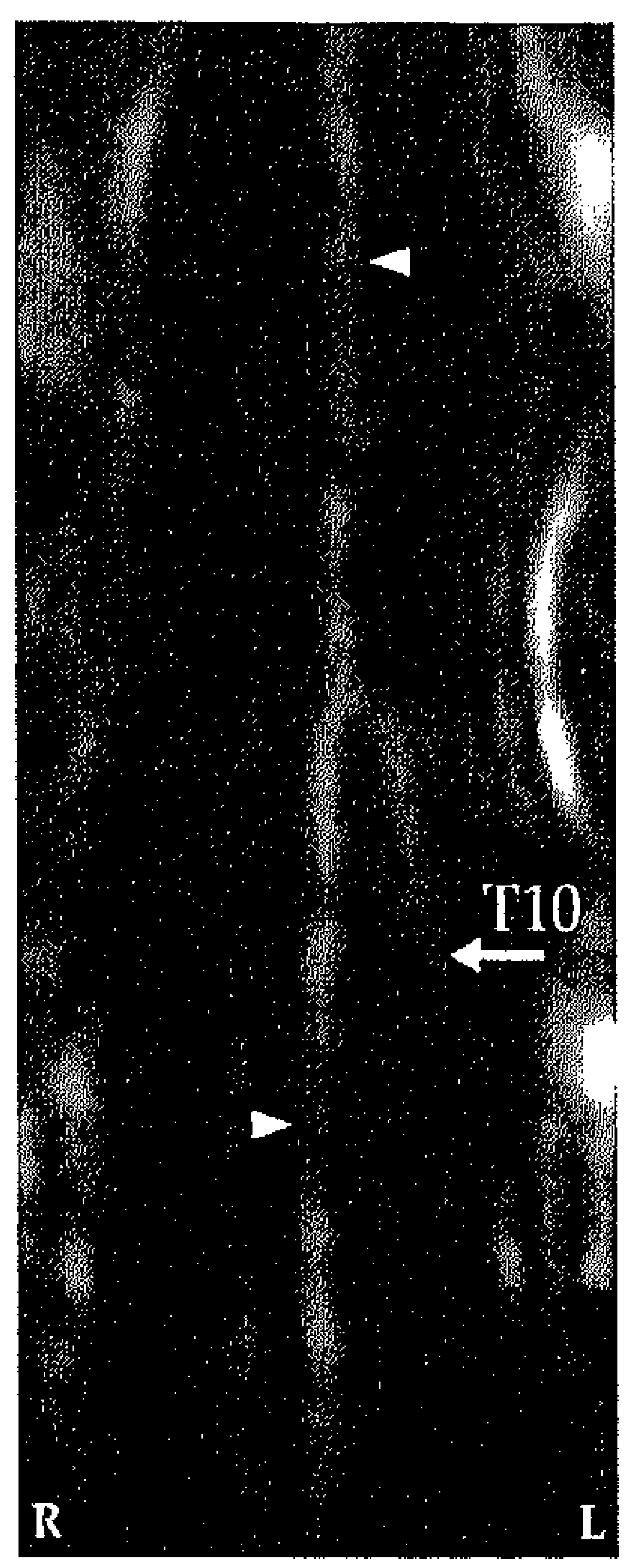

b

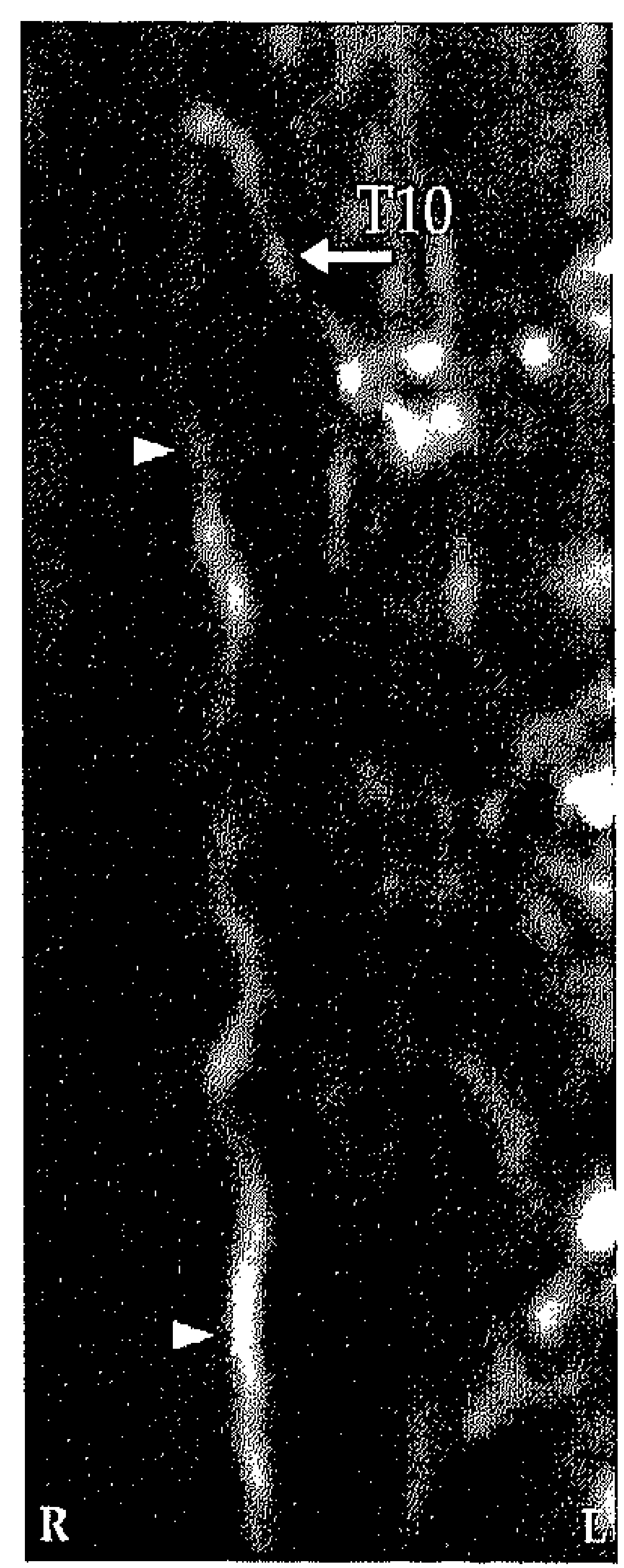

$\mathrm{C}$

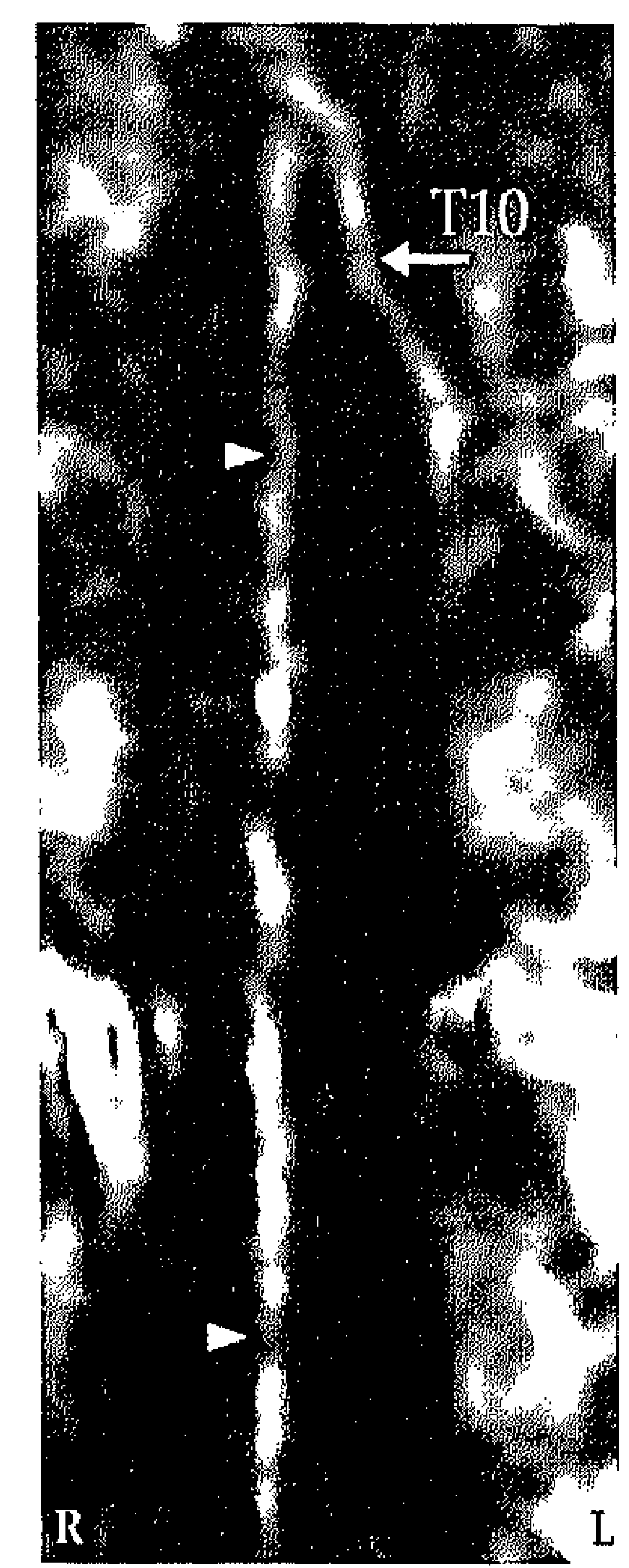

d

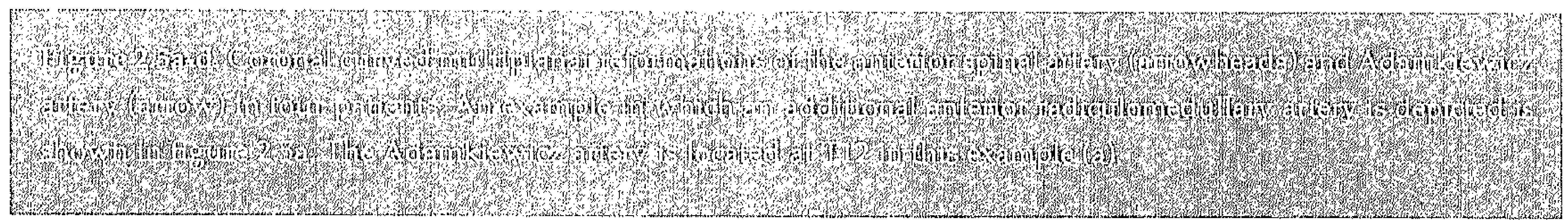


ASA may be visualized. This suggests that all the AKAs observed in our patient group are at least $0.3 \mathrm{~mm}$ in diameter. The ASA could not be followed all the way from T5 down to L5 in all patients.

Particularly in the high and middle part of the thoracic segments, the ASA was not observed. The ASA has the smallest diameter in this part of the spine and is therefore also most vulnerable to ischemia. According to our phantom study, the diameter of the ASA is expected to be less than $0.3 \mathrm{~mm}$ in these segments. Another reason why depiction of the ASA in the high and middle thoracic segments was not achieved is that this area is associated with the most image artefacts caused by heart motion.

Results of our study with consideration of the lateralization ( $75 \%$ left) and level (all between T9 and L2) of the AKA origin are in excellent agreement with findings in literature on vascular anatomy. In a postmortem study in 102 persons, Koshino et $\mathrm{al}^{9}$ reported a leftsided origin of the AKA in $70 \%$. In $91 \%$ of all patients, the AKA arose from a vertebral level between T8 and L1. Bowen et al ${ }^{14}$ found that the AKA arose between T9 and T12 in 62-75\% of patients.

In our study, a higher percentage (100\%) for detection of the AKA and ASA was found than was previously reported in publications $^{11-13}$ about MR angiography. Although the number of included patients was limited, several explanations can be given. These are that a larger FOV covering L5 up through T5 was used, that a higher arterial contrast agent concentration was employed, and that there was an absence of gross vascular pathology in the patient population of the study.

In this study, a larger craniocaudal FOV $(450 \mathrm{~mm})$ than that used in the studies of
Yamada et al ${ }^{11,12}$ (FOV, 280-300 mm) and of Yoshioka et al13 (FOV, $240 \mathrm{~mm}$ ) was possible with the use of a phased-array (whole) spine coil. This means that a larger body region was investigated to locate the AKA. In our study, spatial resolution was comparable with that in the study of Yamada et al11,12.

Yoshioka et $\mathrm{a}^{13}$ achieved a voxel size that was two times smaller with use of a phasedarray spine coil, but the FOV they used was almost half the size of the FOV we used. That improved imaging was achieved in our study can also be noted from the additional small vessels that were depicted. In two patients, more than one anterior radiculomedullary artery was found, and this artery is by definition thinner than is the AKA. Moreover, in two patients, posterior spinal arteries were observed, which are thinner than the ASA.

Synchronization of peak arterial contrast agent concentration with acquisition of central $k$-space profiles is one of the most important aspects of MR angiography. When one starts imaging too early or too late, a great deal of signal will be lost. In the current study, an elliptic-centric manner of $k$-space filling was applied. First, the center of $\mathrm{k}$-space was filled, and this process provided the main signal contrast to the desired vessels. Later, the outer $\mathrm{k}$-space regions were filled, and this process determined the spatial details of the imaged structures. The time of bolus arrival varied within at least 5 seconds among patients, and this variation was sufficiently large to allow degradation of image contrast.

We used the same amount of contrast agent but a faster injection speed $(3 \mathrm{~mL} / \mathrm{sec})$ than that $(0.8-2 \mathrm{~mL} / \mathrm{sec})$ used in the study of Yamada et al11,12. When one compares the injection protocol of Yoshioka et $\mathrm{al}^{13}$ with that used in our study, one may observe that they 
used a smaller dose of contrast agent $(0.2$ $\mathrm{mmol} / \mathrm{kg})$ and a far slower injection rate $(0.2$ $\mathrm{mL} / \mathrm{sec}$ ). Our faster injection speed was chosen to provide a higher first-pass arterial concentration of contrast agent before $\mathrm{T} 2{ }^{*}$ effects dominated, and thus, these features yielded improved arterial T1 signal. A high bolus concentration fits to the centric k-space filling order and the short acquisition time (less than 40 seconds) to allow separation of arterial from venous enhancement. Slow injection speeds allow longer acquisition times (in the study of Yoshioka et $\mathrm{al}^{13}$, 5-6 minutes), but they provide angiographic images in which both arteries and veins are equally enhanced. The triple dose of contrast agent in combination with the fast injection rate, as used in the current study, is reported to be safe ${ }^{16,17}$.

In our study, another reason for the larger percentage of observed AKAs is that our patients did not have any symptomatic vascular disease or insufficiency. The presence of atherosclerotic plaques may contribute to degradation of visualization of the AKA and ASA, because reduction of the arterial lumen diameter and flow hampers contrast agent arrival.

An alternative modality to MR angiography for visualization of spinal vasculature is computed tomographic (CT) angiography. Kudo et al ${ }^{18}$ used CT angiography in patients with known liver disease or in those who were suspected of having it but who did not have vascular pathologic findings. They found that the AKA was visualized in a limited craniocaudal FOV in 13 (68\%) of 19 patients. Takase et $\mathrm{al}^{19}$ used $\mathrm{CT}$ angiography and a larger FOV in patients with thoracoabdominal vascular pathologic findings and found this artery in $63(90 \%)$ of 70 patients. Yoshioka et al ${ }^{13}$ compared results obtained with $C T$ angiography (24 of 30 patients) and MR angiography (20 of 30 patients) in patients with thoracoabdominal aneurysms and concluded that both techniques are suitable.

Potential benefit of $M R$ angiography for thoracoscopic microdiscectomy

In thoracoscopic surgery for herniated disk, several factors determine the surgeon's choice of a left-sided or a right-sided approach. These are as follows: the mediolateral localization of the herniated disk, the vertebral level, the presence of an elongated or dilated aorta covering the left lateral surface, and previous transthoracic surgery that caused pleural adhesions. The choice of the side of approach is also hampered by the presence of anatomic structures such as the liver. Therefore, it is difficult to perform surgery from the right side at T9 and downward. Most thoracic herniated disks occur at these lower thoracic levels and are thus preferentially approached from the left side, where the AKA is most often located.

Segmental vessels are frequently traumatized or sacrificed, and this result leads to a broadening of the surgical field around the pathologically affected disk. This might damage the blood supply to the AKA, which in turn may lead to spinal cord ischemia, when possible collateral circulation fails. Exact knowledge of the segmental supplying level would permit the surgeon to alter his or her surgical strategy whenever necessary. In cases in which the segmental artery that gives rise to the AKA runs over one of the vertebral bodies in the surgical field, it is at risk to be accidentally or even deliberately coagulated during surgery. In three of eight patients (herniated disk at levels T10 through T12), the 
initially planned approach through the left hemithorax was abandoned, and a right-sided approach was chosen instead. The surgeon thus avoided surgical contact with the AKA supplier. In all three patients in whom the approach was changed, surgery and the postoperative course were uneventful. In the other five patients, the AKA originated above or below the level of the vertebrae that had to be partially dissected, so no alteration of side of approach had to be made.

\section{Limitations}

Our results, with consideration of the detectability of the spinal arteries, must be interpreted with caution. First, the number of included patients was small, and results in studies with larger groups of patients are needed to verify our results. Furthermore, our phantom model study does not represent a detailed physiologic situation. Flow effects of the cerebrospinal fluid were not taken into account and may contribute to deterioration of the spatial detection limits. The surrounding tissue of the anterior spinal artery, the anterior radiculomedullary arteries, and the segmental arteries is different and not homogeneous. We used a fluid with T1 of $800 \mathrm{msec}$ (approximately equivalent to muscle tissue) to represent a worst-case situation. Because cerebrospinal fluid surrounds the spinal vessels, the in vivo detection would yield depiction of vessel diameters that could not be seen in the phantom. Use of a T1 representative of cerebrospinal fluid would more closely reflect the surrounding environment of the anterior spinal artery and would have yielded better detection results for our phantom study but would not have been representative of the anterior radiculomedullary arteries and the segmental arteries.
In addition, the concentration of the contrast agent is static in the phantom and does not reflect the bolus dynamics in the arteries. The tube walls (plastic material) yield zero signal contribution in the partial-volume effects, and this result reduces the detected signal in the smaller tubes. The influence of these shortcomings might have diminished our results concerning spatial resolution. The use of a tube with a $0.3 \mathrm{~mm}$ diameter, which was not available, represents the crossing between signal of the tube and that of surrounding fluid and is just an indication that vessel diameters smaller than the voxel sizes can be detected. In conclusion, we demonstrated that MR angiography aids in the visualization of the feeding arteries of the lower spinal cord and that visualization is important in the preoperative planning of thoracoscopic surgery for herniated disk.

\section{Acknowledgements}

We are grateful to Martin van der Wolf of Instrument Development Engineering and Evaluation, Maastricht, the Netherlands, for designing and constructing the vessel phantom. We thank Etienne Lemaire for technical support and advice during the MR angiographic procedure. 


\section{REFERENCES}

1. Anderson TM, Mansour KA, Miller JI, Jr. Thoracic approaches to anterior spinal operations: anterior thoracic approaches. Ann Thorac Surg 1993;55:1447-51; discussion 51-2.

2. Stillerman CB, Chen TC, Couldwell WT, Zhang W, Weiss $\mathrm{MH}$. Experience in the surgical management of 82 symptomatic herniated thoracic discs and review of the literature. I Neurosurg 1998;88:623-33.

3. Champlin AM, Rael J, Benzel EC, et al. Preoperative spinal angiography for lateral extracavitary approach to thoracic and lumbar spine. AJNR Anl / Neuroradiol 1994;15:73-7.

4. Lu J, Ebraheim NA, Biyani A, Brown JA, Yeasting RA. Vulnerability of great medullary artery. Spine 1996;21:1852-5.

5. Anand N, Regan JJ. Video-assisted thoracoscopic surgery for thoracic disc disease: Classification and outcome study of 100 consecutive cases with a 2 -year minimum follow-up period. Spine 2002;27:871-9.

6. Naunheim KS, Barnett MG, Crandall DG, Vaca KJ, Burkus JK. Anterior exposure of the thoracic spine. Ann Thorac Surg 1994;57:1436-9.

7. Rosenthal D. Endoscopic approaches to the thoracic spine. Eur Spine J 2000;9 Suppl 1:S8-16.

8. Rosenthal D, Dickman CA. Thoracoscopic microsurgical excision of herniated thoracic discs. I Neurosurg 1998;89:224-35.

9. Koshino T, Murakami $G$, Morishita $K$, Mawatari $T$, Abe $\mathrm{T}$. Does the Adamkiewicz artery originate from the larger segmental arteries? J Thorac Cardiovasc Surg 1999;117:898-905.

10. Kieffer E, Fukui S, Chiras J, Koskas F, Bahnini A, Cormier E. Spinal cord arteriography: a safe adjunct before descending thoracic or thoracoabdominal aortic aneurysmectomy. J Vasc Surg 2002;35:262-8.

11. Yamada N, Okita $Y$, Minatoya $K$, et al. Preoperative demonstration of the Adamkiewicz artery by magnetic resonance angiography in patients with descending or thoracoabdominal aortic aneurysms. Eur / Cardiothorac Surg 2000;18:104-11.

12. Yamada N, Takamiya $M$, Kuribayashi S, Okita $Y$, Minatoya K, Tanaka R. MRA of the Adamkiewicz artery: a preoperative study for thoracic aortic aneurysm. / Comput Assist Tomogr 2000;24:362-8.

13. Yoshioka $K$, Ninuma $H$, Ohira $A$, et al. MR angiography and CT angiography of the artery of Adamkiewicz: noninvasive preoperative assessment of thoracoabdominal aortic aneurysm. Radiographics 2003;23:1215-25.

14. Bowen BC, DePrima S, Pattany PM, Marcillo A, Madsen $P$, Quencer RM. MR angiography of normal intradural vessels of the thoracolumbar spine. AJNR An J Neuroradiol 1996;17:483-94.

15. Maki JH, Prince MR, Chenevert TC. Optimizing threedimensional gadolinium-enhanced magnetic resonance angiography. Original investigation. Invest Radiol 1998;33:528-37.

16. Cochran ST, Bomyea K, Sayre JW. Trends in adverse events after IV administration of contrast media. $A J R$ Am I Roentgenol 2001;176:1385-8.

17. Boos M, Scheffler K, Haselhorst R, Reese E, Frohlich J, Bongartz GM. Arterial first pass gadolinium-CM dynamics as a function of several intravenous saline flush and Gd volumes. I Magn Reson lmaging 2001;13:568-76.

18. Kudo K, Terae S, Asano T, et al. Anterior spinal artery and artery of Adamkiewicz detected by using multidetector row CT. AJNR Am J Neuroradiol 2003;24:13-7.

19. Takase K, Sawamura $Y$, Igarashi $K$, et al. Demonstration of the artery of Adamkiewicz at multidetector row helical CT. Radiology 2002;223:39-45. 


\title{
Chapter III
}

Comparison of 0.5-M Gd-DTPA with 1.0-M

Gadobutrol for MR angiography of the supplying arteries of the spinal cord in thoracoabdominal aortic aneurysm patients

\author{
RJ Nijenhuis \\ $S$ Gerretsen \\ T Leiner \\ MJ Jacobs \\ JMA van Engelshoven \\ WH Backes
}

Journal of Magnetic Resonance Imaging 2005;22:136-44 


\section{ABSTRACT}

Purpose: To prospectively compare $0.5-\mathrm{M}$ gadopentetate dimeglumine (Gd-DTPA) with 1.0-M Gadobutrol for magnetic resonance (MR) angiography of the blood supplying arteries of the spinal cord in patients referred for open surgical repair of a thoracoabdominal aortic aneurysm (TAAA).

Materials and Methods: A total of 11 patients with a TAAA underwent two threedimensional $M R$ angiography exams of the aorta, segmental arteries (SAs), artery of Adamkiewicz (AKA), and anterior spinal artery (ASA). Imaging was performed on two separate occasions using Gd-DTPA and Gadobutrol as contrast agents at $0.3 \mathrm{mmol} / \mathrm{kg}$. Images were evaluated by measuring signalto-noise (SNR) and contrast-to-noise (CNR) ratios and were judged for different image quality criteria by two blinded observers.

Results: In all patients both MR angiography exams were of sufficient image quality to detect the AKA and ASA. No significant differences in SNR and CNR were observed between the two contrast agents. According to the observers, no significant differences in subjective image quality were found.

Conclusions: Using both contrast agents it was possible to visualize the ultrasmall spinal cord arteries in all cases. The use of the 1.0-M contrast agent did not improve image quality of MR angiography images of the blood supplying arteries of the spinal cord compared to the $0.5-\mathrm{M}$ contrast agent. 


\section{INTRODUCTION}

In thoracoabdominal aortic aneurysm (TAAA) surgery, preoperative knowledge of the spinal cord vascular supply may be of importance to prevent paraplegia ${ }^{1}$. Best image quality to depict the blood supplying arteries of the spinal cord may be obtained using intraarterial catheter ( $X$-ray) angiography, also known as digital subtraction angiography. Catheter angiography is, however, not generally used in TAAA patients, as selective catheterization of feeding segmental arteries (SAs) may cause thromboembolisms, possibly resulting in paraplegia ${ }^{2}$.

Recently, magnetic resonance (MR) angiography has emerged as a possible alternative for visualizing the arterial pathway to the spinal cord. Although the reported percentages in which the artery of Adamkiewicz (AKA) could be detected in TAAA patients did not exceed $83 \%^{3-6}$, MR angiography has several advantages over catheter angiography. These advantages are the minimally invasive approach of MR angiography, the nontoxicity of the gadolinium-based contrast agent, and the absence of radiation exposure. Therefore optimization of MR angiography for spinal cord vasculature is of interest.

The arterial pathway to the spinal cord includes the aorta, several SAs, one or more anterior radiculomedullary arteries (ARAs), and finally the anterior spinal artery (ASA). The largest ARA that contributes to the ASA is the AKA, which is considered to be the most important artery for the blood supply of the lower spinal cord.

There are many aspects that hamper the visualization of the arteries of the spinal cord. A high spatial resolution is required to depict the small caliber spinal cord arteries, with a diameter that is subject to substantial intraand interindividual variation (range 0.2-0.8 $\mathrm{mm})^{7,8}$. Moreover, a large field of view (FOV) is required, as the AKA may arise from a variable segmental location comprising the entire thorax and abdomen ${ }^{7,8}$. Because there is high similarity in the configuration, pathway, and localization of the SAs, spinal cord arteries, and veins, a clear distinction between them is difficult when solely judged on anatomic features. Therefore, when separation between arteries and veins is desired using MR angiography, a high temporal resolution is necessary. However, simultaneous high spatial resolution, high temporal resolution, and a large FOV are conflicting requirements in MRI. To overcome these difficulties, firstpass T1-weighted MR angiography with a high dose of contrast agent seems the best choice to depict the arterial pathway to the spinal cord. Ideally, one could improve image quality by using a contrast agent with stronger $\mathrm{T} 1$ shortening properties. Using a high dose of gadopentetate dimeglumine (Gd-DTPA), a $0.5-\mathrm{M}$ contrast agent, it has already been shown that the spinal cord vasculature can be visualized ${ }^{3-6,9}$. Interestingly, Goyen et al ${ }^{10,11}$ showed that Gadobutrol, a 1.0-M agent, gave much better contrast-to-noise (CNR) and better delineation of small peripheral arteries $^{11}$ than Gd-DTPA, due to a higher T1 relaxivity.

The purpose of this study was to investigate whether Gadobutrol would yield improved image quality for the detection of the arterial pathway to the spinal cord in comparison to Gd-DTPA using MR angiography in TAAA patients. To this end, we compared the image quality of both contrast agents in the same TAAA patients, and furthermore determined the intrinsic in vitro 
Table 3.1. Intrinsic properties and administration of contrast agents.

[M]
$\begin{aligned} & \text { Gadopentetate } \\ & \text { dimeglumine }\end{aligned}$
Gadobutrol
$\left[\mathrm{mM}^{-1} \mathrm{sec}^{-1}\right]$

\section{MATERIALS and METHODS}

\section{Patients}

Between November 2002 and September 2003, 11 TAAA-patients (eight male) were included for imaging prior to elective aortic replacement. All patients (age $67 \pm 5$ years; mean $\pm \mathrm{SD}$ ) underwent two MR angiography exams. The time between the two exams was two days for all patients. The time between the last MRI exam and the operation was one day for all patients. The medical ethics commission of the hospital approved the protocol, and written informed consent was obtained from all patients, prior to their inclusion.

\section{Contrast agents}

The contrast agents used in this study were Gd-DPTA (Magnevist; Schering, Berlin, Germany) and Gadobutrol (Gadovist; Schering, Berlin, Germany). The injection rates of both agents were matched to $1.5 \mathrm{mmol} / \mathrm{sec}$, to obtain a similar first-pass peak arterial concentration in the aorta. In both MR angiography exams the left antecubital vein was

$\begin{array}{ccr}\begin{array}{c}\mathrm{r}^{*} \\ {\left[\mathrm{mM}^{-1} \mathrm{sec}^{-1}\right]}\end{array} & \begin{array}{c}\text { Injection dose } \\ {[\mathrm{mL}]}\end{array} & \begin{array}{r}\text { Injection } \\ {[\mathrm{mLsec}}\end{array} \\ 4.7 & 45 & 3.0 \\ 5.4 & 22.5 & 1.5\end{array}$

punctured to inject the contrast medium. Contrast agent properties as well as details of the injection protocol are listed in Table 3.1. Contrast agent administration was followed by $25 \mathrm{~mL}$ of saline flush, injected at the same injection rate as the contrast agent.

\section{MR imaging}

All acquisitions were performed using a clinical 1.5-Tesla MRI system, (Philips Intera, software release 8; Philips Medical Systems, Best, The Netherlands) in combination with a phased-array surface spine coil. Patients were imaged in the supine position. Each study consisted of three acquisitions. First, T2weighted anatomic survey scans were performed to image the course of the spinal cord and served for anatomic reference.

The field of view (FOV) for imaging covered the fifth thoracic vertebra (T5) down to the first sacral vertebra (S1) to ensure that the AKA was in the FOV. The survey scans were acquired in axial and sagittal orientations. Acquisition parameters were TR 2686 msec; TE $120 \mathrm{msec}$; flip angle $90^{\circ}$; matrix size $320 \times 480$ (phase encoding $\times$ readout); 12 slices; and voxel sizes $1.25 \times 1.67 \times 4.0 \mathrm{~mm}$.

MR fluoroscopy was used to determine the optimal scan delay for the start of the MR angiography acquisition. The obtained scan 
delay was used to synchronize the sampling of the center of k-space with the peak contrast agent concentration in the subsequent MR angiography acquisition. For this purpose a test bolus of $2.0 \mathrm{~mL}$ was administered for GdDTPA and $1.0 \mathrm{~mL}$ for Gadobutrol. The test bolus was injected at the same rate (Table 3.1) as the final bolus using a power-injector (Spectris; Medrad, Indianola, PA, USA), followed by a saline flush of $25 \mathrm{~mL}$. The used pulse sequence consisted of a single $80-\mathrm{mm}$ thick sagittal slice, which was placed through the aorta. The single slice was acquired once each second over a period of two minutes. The pulse sequence parameters were TR $5.2 \mathrm{msec}$; TE $1.4 \mathrm{msec}$; flip angle $35^{\circ}$; FOV $450 \mathrm{~mm}$; and matrix size $205 \times 256$. Viewing the series of bolus timing images, the scan delay was defined as the time between start of test bolus injection and maximal enhancement of the entire aorta down to the bifurcation.

Each MR angiography consisted of one dynamic phase lasting between 36 and 40 seconds, depending on the number of slices. Imaging was performed without breath-hold. FOV size in craniocaudal direction (frequency encoding) was $45 \mathrm{~cm}$, and in the anteriorposterior direction (phase encoding) $16 \mathrm{~cm}$, and matrix size $464 \times 512$.

Acquired sagittal slices were $1.2 \mathrm{~mm}$ thick and interpolated (zero-filling in k-space) during reconstruction to $0.6 \mathrm{~mm}$ thick slices. The number of slices was individually adjusted (range 70-80) to include the entire vertebral cross-section over the total craniocaudal FOV (T5-S1). Acquisition parameters were TR $5.9 \mathrm{msec}$; TE $1.9 \mathrm{msec}$ (partial echo $62.5 \%$ ); and flip angle $30^{\circ}$. Acquired pixel size was $0.8 \times 0.8 \mathrm{~mm}$. To obtain images with minimal venous enhancement, k-space was filled using elliptical centric ordering in which the first 4 seconds were randomly filled (CENTRA; Philips Medical Systems, Best, The Netherlands).

\section{Image Analysis}

The following criteria were applied to identify the ASA, the AKA, and the connecting SA: (i) the ASA was identified as an enhanced structure lying in the middle of the anterior surface of the spinal cord; (ii) an enhanced vessel coursing through the intervertebral foramen towards the spinal cord, with a steep ascending feature and connecting to the spinal artery at the top of the hairpin curve was considered to be the AKA; (iii) the vertebral level of origin of the AKA was confirmed using the T2-weighted anatomic scan. The intervertebral level at which the segmental artery entered the spinal cord was defined as the level of origin. For instance, in case the SA entered between T12 and the first lumbar (L1) vertebra, this was considered to be the T12 SA (similar to the nerves); (iv) only when the enhanced vessel originated between $\mathrm{T} 9$ and L2 was it considered to be the AKA. If more enhanced vessels originated between $\mathrm{T} 9$ and L2, the one with largest (i.e., thickest) diameter was considered to be the AKA and arteries of smaller diameter (i.e., thinner) were considered to be other ARAs.

To objectively quantify image quality, signal intensity (SI) and image sharpness measurements were performed. Interobserver agreement with regard to image quality of the two MR exams was used as subjective image quality measure.

For objective image quality, SI measurements were performed using user-defined regions of interest (ROIs) in the different vessels at different vertebral levels in all 
subjects. The examined vessel segments were: (i) the aorta at the levels T3-T7, T8-T12, and L1-L5; (ii) the thickest SA was examined at the levels T3-T7, T8-T12, and L1-L5; (iii) the SAs that contributed to the AKAs; (iv) all the visualized AKAs; and (v) the ASA at the levels T3-T7, T8-T12, and L1-L5. The aorta, thickest SA, and ASA were examined at multiple levels because these vessels run through the entire FOV. The size of the ROI was chosen such that as much of the vascular lumen was covered as possible. ROI sizes varied from $1.5 \mathrm{~mm}^{2}$ for the ASA to $25 \mathrm{~mm}^{2}$ for the aorta. SI was also measured, using a ROI of equal size, in the tissue immediately adjacent to the artery. Because a phased-array surface coil was used, no representative air regions were available for reliable noise measurements. Therefore, the SD of the SI in the erector spinae muscle (SDO) was taken to represent the noise level. SNR was calculated as the ratio between the SI of the artery and SD0. CNR was calculated as the difference between SI of the artery and the surrounding tissue and divided by SDO.

To quantify sharpness of the ASA, the fullwidth-half-maximum (FWHM) was determined from an intensity profile perpendicular through the vessel. The ASA was chosen for this purpose, because this is the smallest artery of interest. FWHM measurements were performed at the exact same level in the two exams of the individual patients. The MR angiography scan that yielded the smallest FWHM value was considered to be the technique with the best (i.e., sharpest) delineation.

Before subjective image quality measurements were carried out, the 22 MR angiography image sets were first blinded for patient details, acquisition date, and contrast agent type. Maximum intensity projections
(MIPs) and multiplanar reformations (MPRs) were used to study the three-dimensional MR angiography image sets. The observers were allowed to use these imaging modalities in every preferred manner. The two observers independently scored the two MR angiography image sets of each patient. The observers had to choose which of the MR angiography scans visualized the ASA and AKA better or equally well. Moreover; they had to point out whether their choice was based on: delineation of the vessel, contrast between vessel and surrounding tissue, homogeneity of the surrounding tissue, or a combination of these criteria. Both image sets were displayed next to each other on a diagnostic computer screen.

\section{Statistical analysis}

For statistical analysis SPSS software was used (version 10.0; SPSS Inc., Chicago, IL, USA) The paired sample Student's t-test was used to test whether the image quality of the Gadobutrol images were better compared to the Gd-DTPA images, according to our hypothesis. Comparisons between the two contrast agents were made for each vessel segment in terms of SNR and CNR. Obtained P-values were corrected for multiple comparisons according to Bonferroni. The FWHM values of the ASA were compared between the two contrast agents. Overall interobserver agreement was calculated using the Cohen's K-value. Statistical significance was inferred when the obtained $P$-value was less than 0.05 . 


\section{Phantom Study}

A phantom study was performed to determine and to compare the intrinsic (i.e., without physiological effects) MRI signal properties of the two contrast agents using the same MR pulse sequence as for the patient study. The phantom consisted of 24 cylindrical reservoirs each with a volume of $50 \mathrm{~mL}$. The reservoirs were filled with human serum at different concentrations: 25, 35, and $50 \mathrm{mM}$. Human serum was used as this is a blood product (in contrast to water) that contains proteins (e.g., albumin), which lower the T1 relaxivity due to protein water interactions. The contrast media used do not have a significant interaction with the proteins or blood cells as they are known to stay in the extracellular space. When injected into the circulation this will yield some additional T1 relaxation (not relaxivity) lowering, which is equal for both agents. The phantom was placed in a water bed, the temperature of which was kept at $37^{\circ} \mathrm{C}$. The electronic water heating device was placed outside the magnet room and was connected to the plastic heater in the waterbed by tubes through which warm water was pumped. Imaging parameters were equal to the patient MR angiography protocol.

For image analysis, SI measurements were performed using a circular ROI (diameter 3.0 $\mathrm{cm}$ ) at three different levels in all the reservoirs. Signal amplifications were calculated as the ratio between the averaged SI of each concentration of the human plasma and the SI at zero concentration. The relation between signal amplification and concentration was numerically fitted to the SI relation of a spoiled gradient echo pulse sequence (Ernst equation).

\section{RESULTS}

\section{Image Analysis}

In all TAAA patients, angiographic data sets of sufficient diagnostic image quality were obtained for both contrast agents. No side effects occurred during or after injection of the contrast agents. In all patients, the aorta, SA, AKA, and ASA were identified. An example of the imaged arterial pathway is given in Figure 3.1. An overview of observations from the MR angiography images is listed in Table 3.2. For both contrast agents, the entire arterial pathway to the spinal cord could be evaluated. The bolus arrivals for Gd-DTPA ( $28 \pm 5$ seconds; mean $上$ SD) was on average four seconds $(P<0.001)$ earlier than for Gadobutrol ( $32 \pm 7$ seconds), due to the faster flushing speed of the saline.

Both observers found that the AKA derived from the left side in $73 \%(8 / 11)$ and from the right side in $27 \%(3 / 11)$ of the patients. The AKA originated in $82 \%(9 / 11)$ of the cases between the vertebrae $\mathrm{T} 9$ and $\mathrm{T} 12$ and in 91\% (10/11) between T8 and L1. Table 3.2 summarizes the results regarding lateralization and craniocaudal localization of the AKA. In nine patients more than one ARA was found. The ASA could be followed in all patients from $\mathrm{T} 9$ down to $\mathrm{L} 2$. In five patients the high thoracic segment T5-T9 of the ASA was not visualized. The identification and craniocatidal localization of the ASA and AKA was identical for the two contrast agents.

In Table 3.3 an overview is given of the SNR and CNR for the different vascular trajectories. Figure 3.2 shows that the measured CNR values differ by a factor of 10 between the different vessel segments and are closely spread around the identity line. When correcting for the multiple comparisons no 


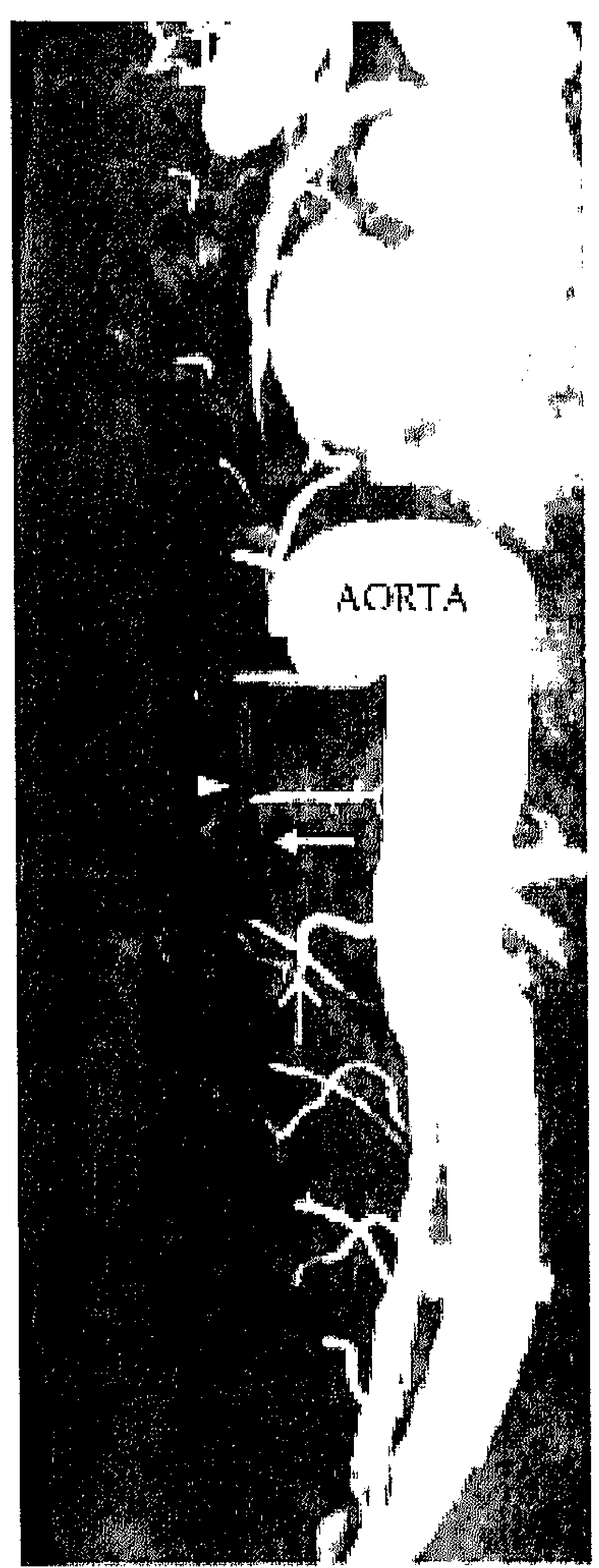

a

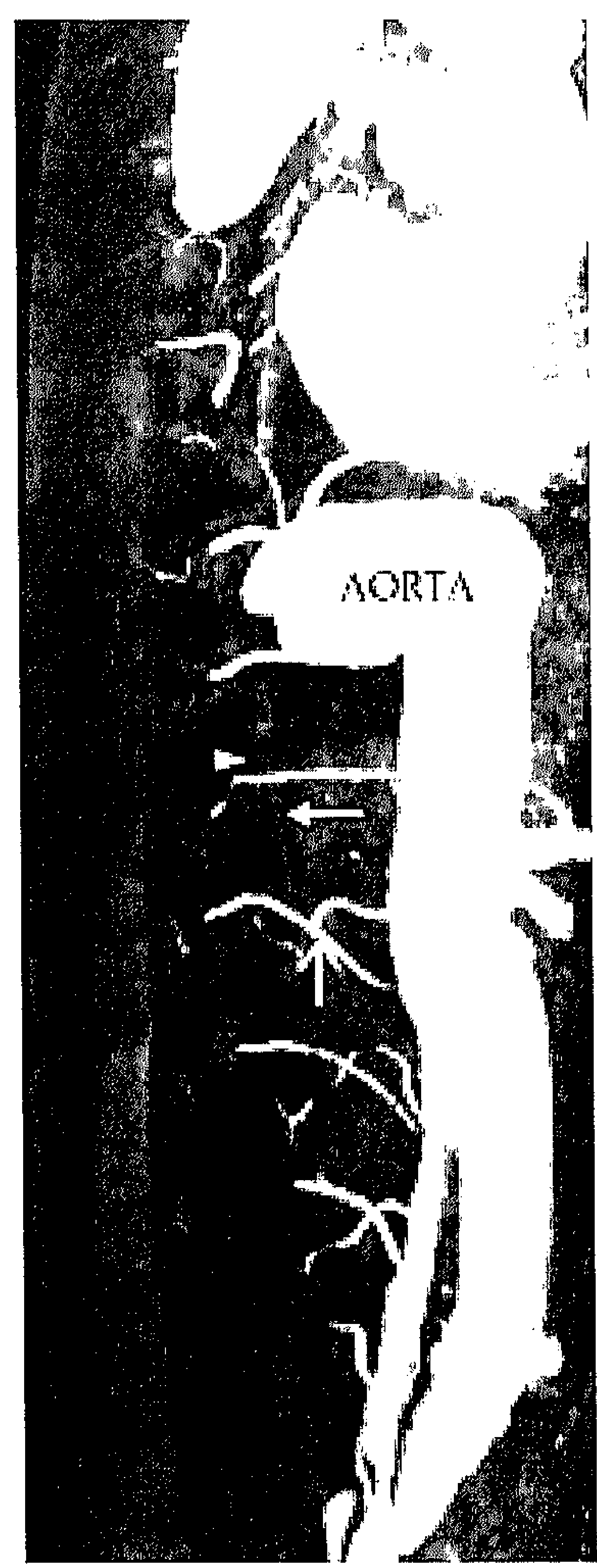

b

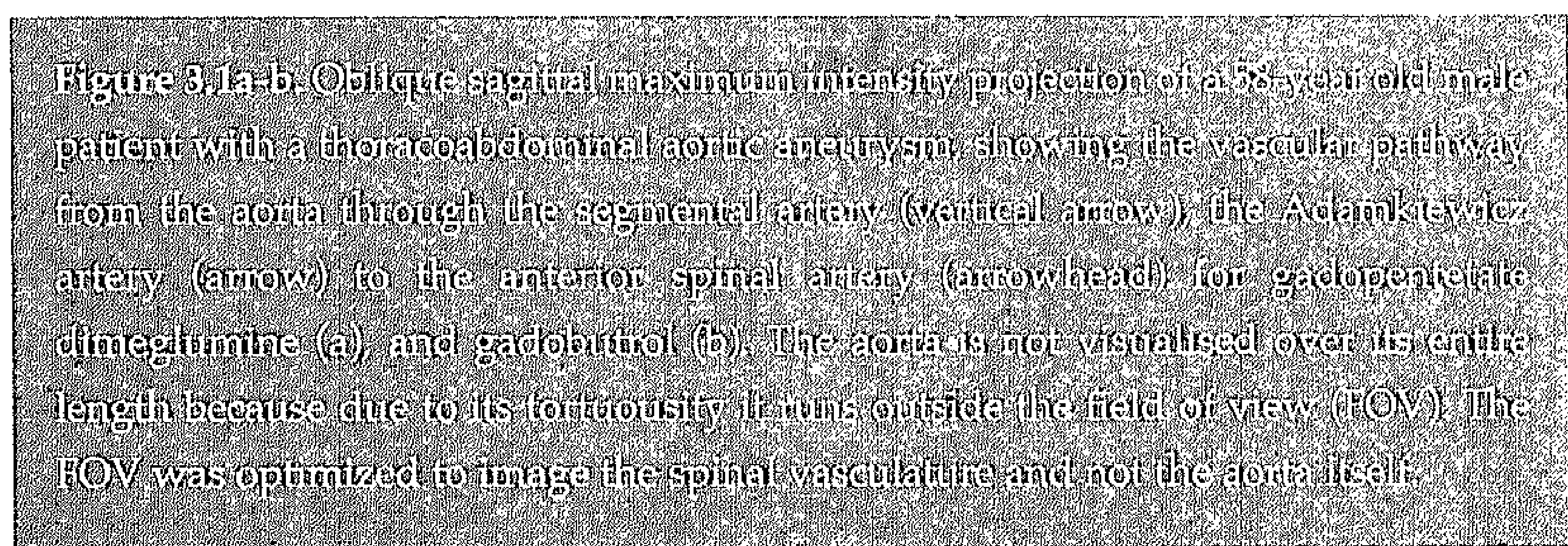

significant $(P<0.3)$ differences between the two contrast agents were found for both the SNR and CNR over all eight analyzed vessel segments.

FWHM calculations revealed a smaller FWHM value of the ASA in six of 11 (55\%) patients for gadopentetate dimeglumine. The overall average value of FWHM was not different $(P<0.3)$ for Gadobutrol $(2.7 \pm 0.3 \mathrm{~mm}$; mean $\pm \mathrm{SD})$ and Gd-DTPA $(2.8 \pm 0.6 \mathrm{~mm})$.

Observer A preferred Gd-DTPA images over the Gadobutrol images in seven of 11 $(64 \%)$ cases. Observer B preferred Gd-DTPA in six of $11(55 \%)$ cases. One case revealed no difference in image quality. In two cases, both observers disagreed. In the first case, observer A found no difference in image quality and observer B preferred the Gadobutrol images. In the second case, observer $B$ preferred the Gd-DTPA images and observer A found no difference in image quality. The overall interobserver agreement as to which of the two contrast agents had the best image quality was $\mathrm{K}=0.62$.

To illustrate the similarity or subtle differences in image quality between both contrast agents examples are given in Figures 3.3, 3.4, and 3.5. 

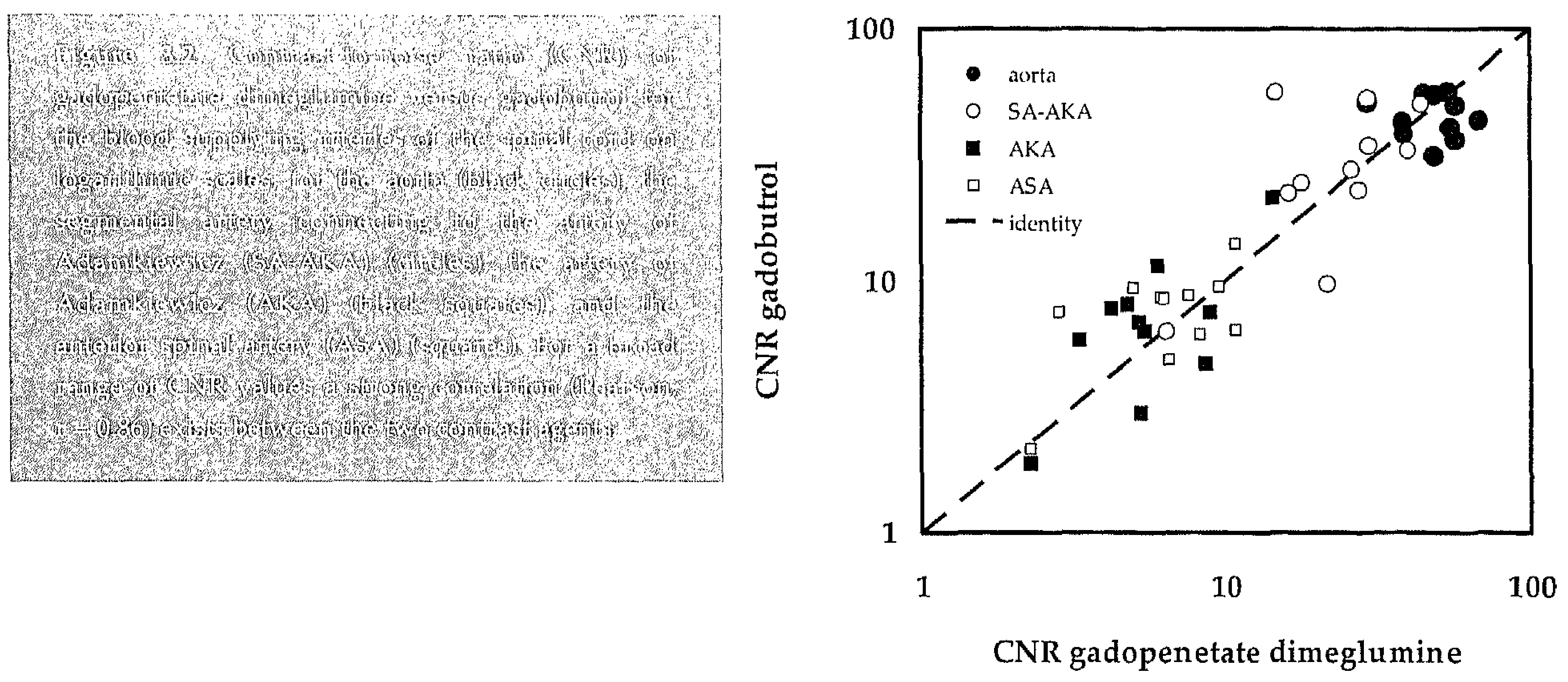

Table 3.2. Overview of observations at MR angiography.

$\begin{array}{cccccc}\text { Patient no. } & \text { Level AKA } & \text { Origin (side) } & \text { Level ARA } & \text { Origin (side) } & \text { ASA visibl } \\ 1 & & & & & \\ 2 & \text { L1 } & \text { Right } & \text { L1 } & \text { Right } & \text { T9 - L2 } \\ 3 & \text { T9 } & \text { Left } & \text { L2 } & \text { Right } & \text { T5 - L2 } \\ 4 & \text { T11 } & \text { Left } & \text { None } & & \text { T5 - L2 } \\ 5 & \text { T12 } & \text { Left } & \text { T8 } & \text { Left } & \text { T9 - L2 } \\ 6 & \text { T12 } & \text { Left } & \text { T10 } & \text { Right } & \text { T5 - L2 } \\ 7 & \text { T10 } & \text { Left } & \text { None } & & \text { T9 - L2 } \\ 8 & \text { T9 } & \text { Right } & \text { T8 } & \text { Left } & \text { T5 - L2 } \\ 9 & \text { T9 } & \text { Left } & \text { L2 } & \text { Left } & \text { T9 - L2 } \\ 10 & \text { T11 } & \text { Left } & \text { L2 } & \text { Left } & \text { T5 - L2 } \\ 11 & \text { T10 } & \text { Left } & \text { L1 } & \text { Left } & \text { T9 - L2 } \\ & \text { L2 } & \text { Right } & \text { T10 } & \text { Left } & \text { T5 - L2 }\end{array}$

$\mathrm{AKA}=\mathrm{Adamkiewicz}$ artery; $\mathrm{ARA}=$ anterior radiculomedullary artery; $\mathrm{ASA}=$ anterior spinal artery; $\mathrm{T}=$ thoracic vertebra; $\mathrm{L}=$ lumbar vertebra 
Table 3.3. Signal-to-noise and contrast-to-noise ratios of the two contrast agents for the different arteries at varyir vertebral levels.

\begin{tabular}{|c|c|c|c|c|c|}
\hline & Artery & $\begin{array}{l}\text { Vertebral } \\
\text { level }\end{array}$ & Gadobutrol & $\begin{array}{l}\text { Gadopentetate } \\
\text { dimeglumine }\end{array}$ & Difference \\
\hline \multirow[t]{8}{*}{ SNR } & Aorta & T3-T7 & $66.4(13.1)$ & $55.7(10.0)$ & $10.7(16.4)$ \\
\hline & & $\mathrm{T} 8-\mathrm{T} 12$ & $54.8(9.2)$ & $59.5(12.7)$ & $-4.7(17.4)$ \\
\hline & & L1-L5 & $60.4(14.3)$ & $63.9(13.5)$ & $-3.5(16.1)$ \\
\hline & ASA & T3-T7 & $7.3(1.9)$ & $5.5(2.3)$ & $1.8(2.3)$ \\
\hline & & T8-T12 & $10.7(3.2)$ & $11.4(3.9)$ & $-0.7(4.1)$ \\
\hline & & L1-L5 & $10.3(4.8)$ & $11.3(5.0)$ & $-1.1(3.2)$ \\
\hline & AKA & & $9.3(3.3)$ & $11.4(6.0)$ & $-2.1(4.9)$ \\
\hline & AKA-SA & & $34.5(11.0)$ & $44.2(19.9)$ & $-9.7(18.8)$ \\
\hline \multirow[t]{8}{*}{$\mathrm{CNR}$} & Aorta & T3-T7 & $56.5(12.1)$ & $46.7(9.5)$ & $9.8(15.1)$ \\
\hline & & T8-T12 & $45.1(8.3)$ & $49.9(11.0)$ & $-4.7(14.4)$ \\
\hline & & L1-L5 & $49.7(13.6)$ & $53.2(12.1)$ & $-3.4(15.4)$ \\
\hline & ASA & T3-T7 & $2.7(2.0)$ & $1.6(1.0)$ & $1.1(2.4)$ \\
\hline & & T8-T12 & $7.0(2.9)$ & $7.7(3.0)$ & $-0.8(2.9)$ \\
\hline & & L1-L5 & $6.4(4.4)$ & $7.3(4.2)$ & $-0.9(2.3)$ \\
\hline & AKA & & $6.0(3.5)$ & $8.0(5.3)$ & $-2.0(3.8)$ \\
\hline & AKA-SA & & $25.3(9.6)$ & $33.6(17.5)$ & $-8.3(16.3)$ \\
\hline
\end{tabular}

$\mathrm{ASA}=$ anterior spinal artery; $\mathrm{AKA}=\mathrm{Adamkiewicz}$ artery; $\mathrm{AKA}-\mathrm{SA}=$ the segmental artery directly supplying the $\mathrm{AK}$ and the ASA; Notation: Mean (SD); $\mathrm{T}=$ thoracic vertebra; $\mathrm{L}=$ lumbar vertebra; $\mathrm{SNR}=$ signal-to-noise ratio; $\mathrm{CNR}$ contrast-to-noise ratio 


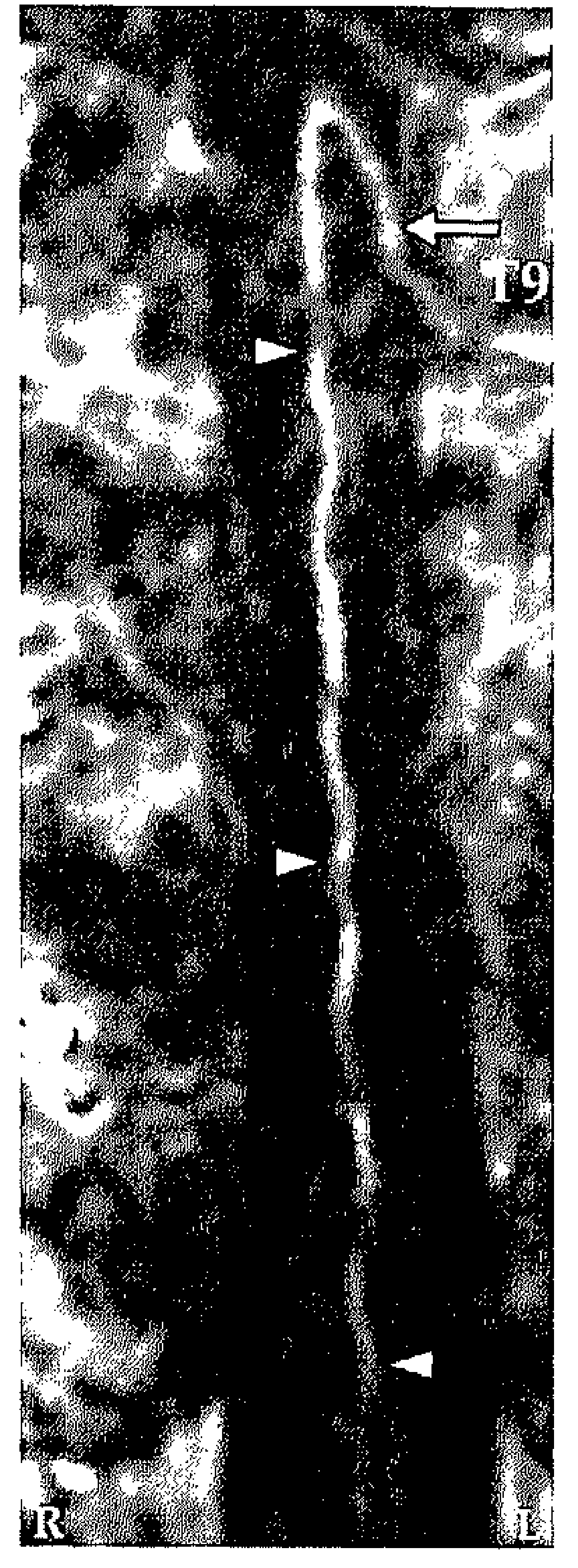

a

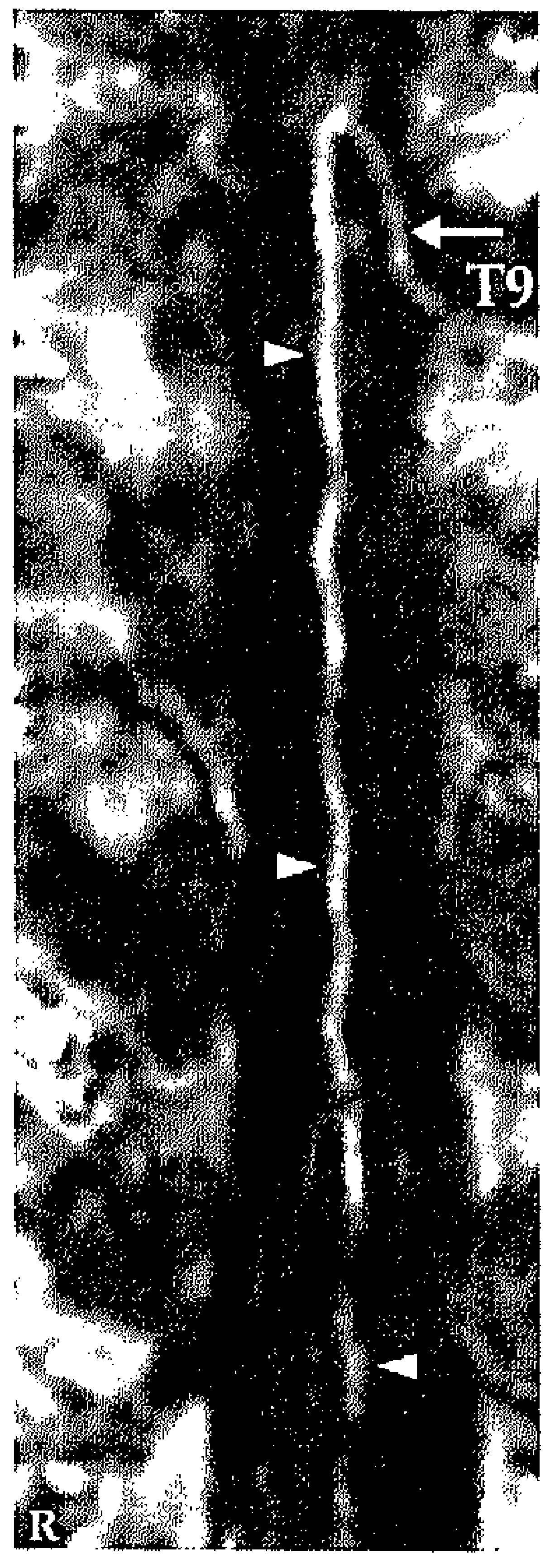

b

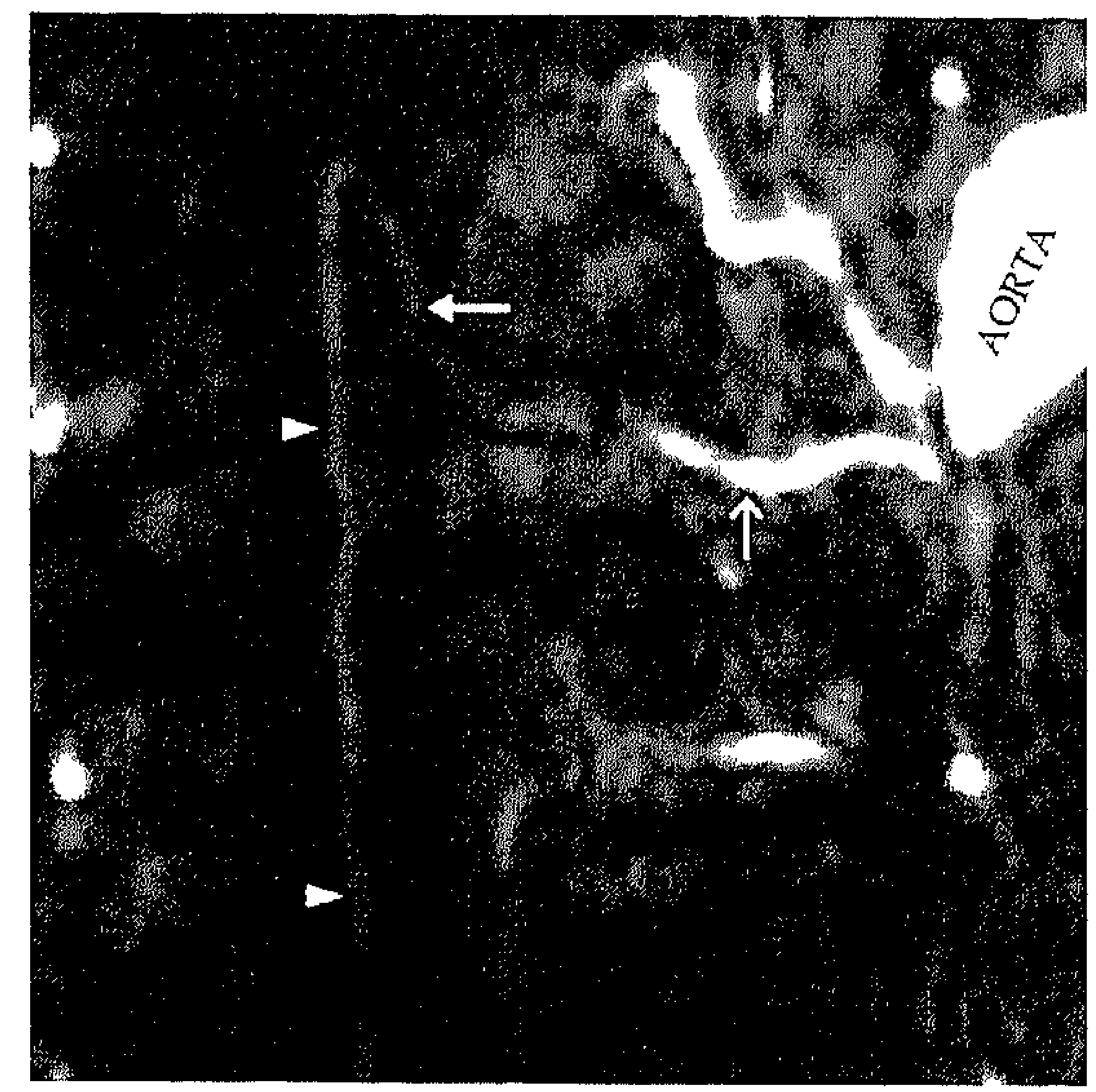

C

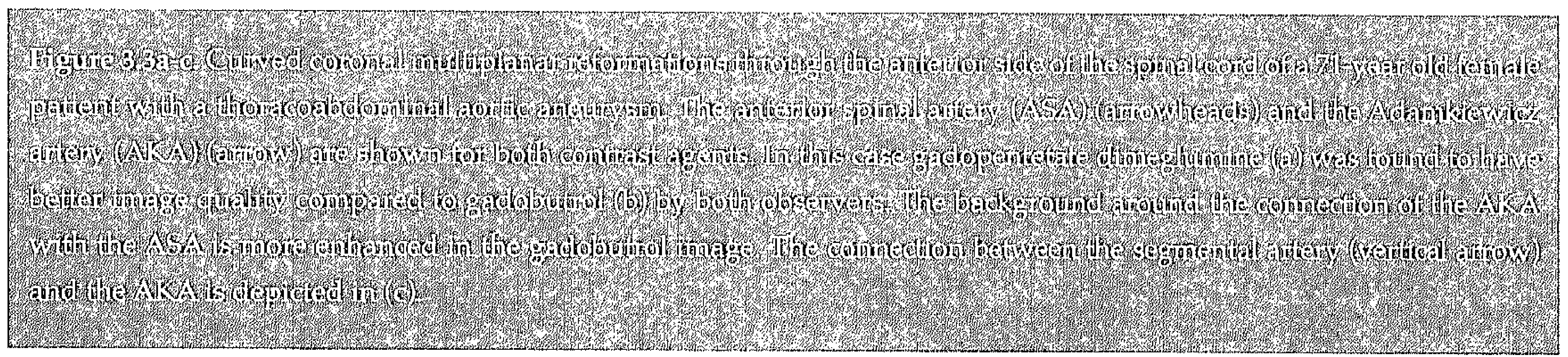

\section{Phantom Study}

Figure 3.6 shows the results of the obtained signal amplifications as a function of the concentration for the two contrast agents. The maximum amplification for Gadobutrol was reached at a lower concentration $(15 \mathrm{mM})$ than for Gd-DTPA (22 mM). Moreover, the maximum was broader for Gd-DTPA than for Gadobutrol. Interestingly, maximum signal amplifications for both agents was equal to 28 . The signal amplification for Gadobutrol appeared to be higher at lower concentrations $(<15 \mathrm{mM})$ than for Gd-DTPA due to the higher T1 relaxivity. Gadobutrol showed a stronger reduction in signal amplification for higher concentrations $(>20 \mathrm{mM})$ due to the higher T2 (or $\mathrm{T}^{*}$ ) relaxivity. 


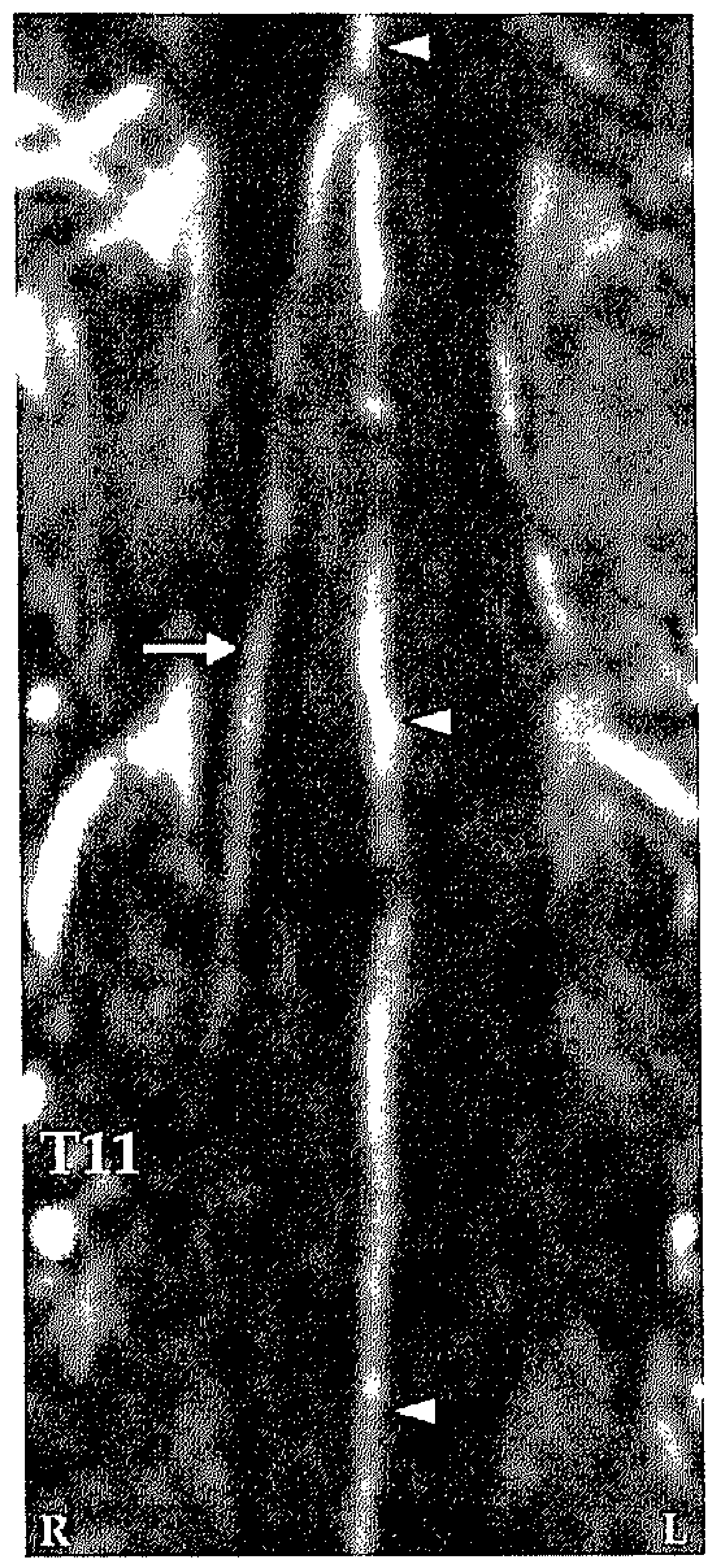

a

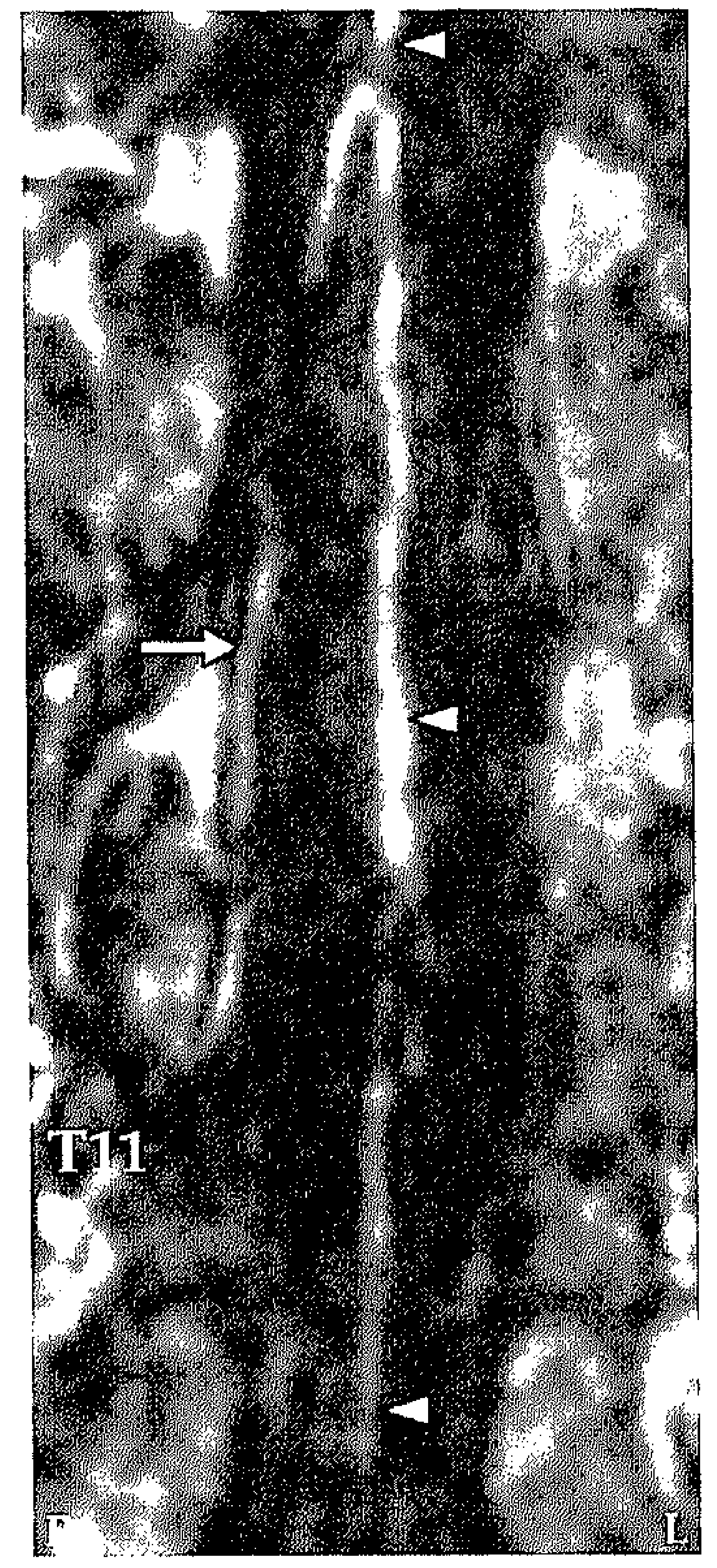

$\mathrm{b}$

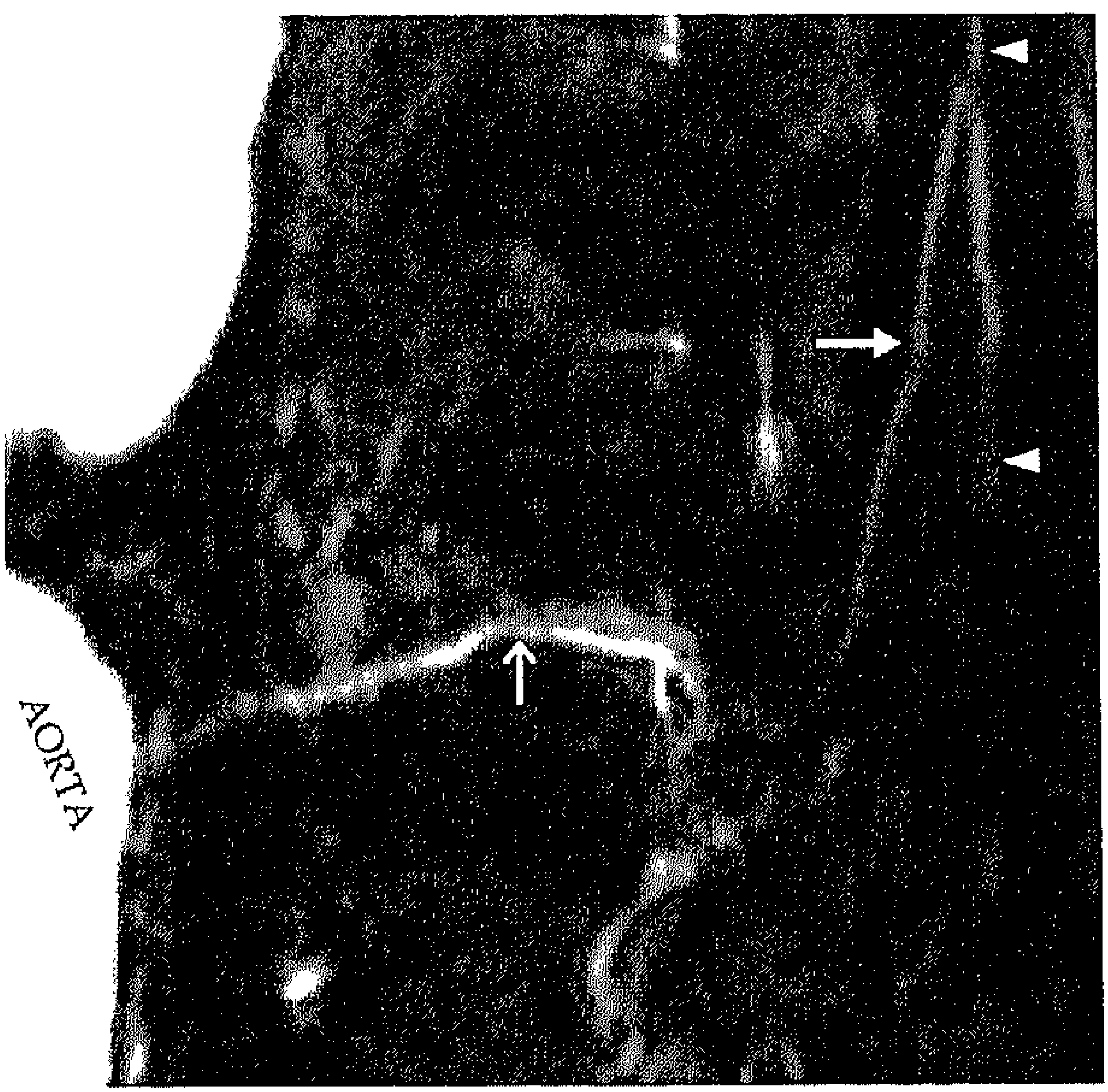

C

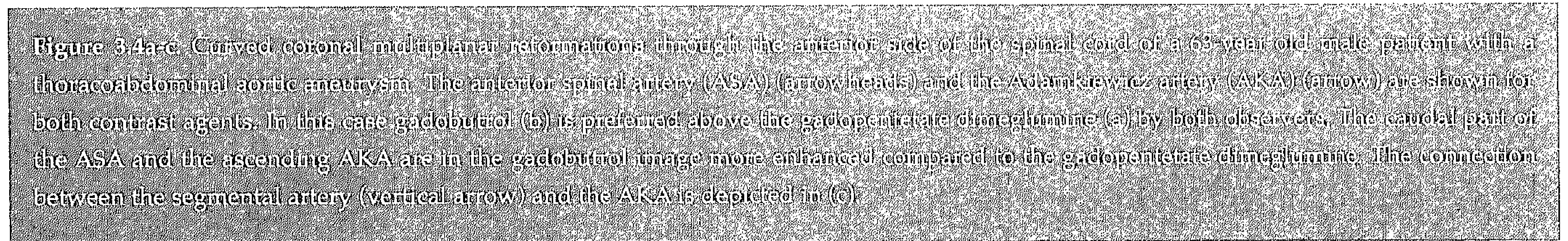

\section{DISCUSSION}

This study showed that there was no difference between a $0.5-\mathrm{M}$ and $1.0-\mathrm{M}$ gadolinium-based contrast agent for depiction of the arteries supplying the spinal cord. In all 11 TAAA patients, the ultrasmall AKA and the thoracolumbar ASA were depicted with high image quality with both contrast agents.
Our results of the AKA considering the lateralization (75\% left and $25 \%$ right) and vertebral level (91\% between T8-L1) compare very well with the literature on vascular anatomy ${ }^{7}$. This is the first study to image the spinal cord arteries two times and therefore shows that spinal cord arteries can be reproducibly imaged using $M R$ angiography. The ASA, however, could not be followed all 


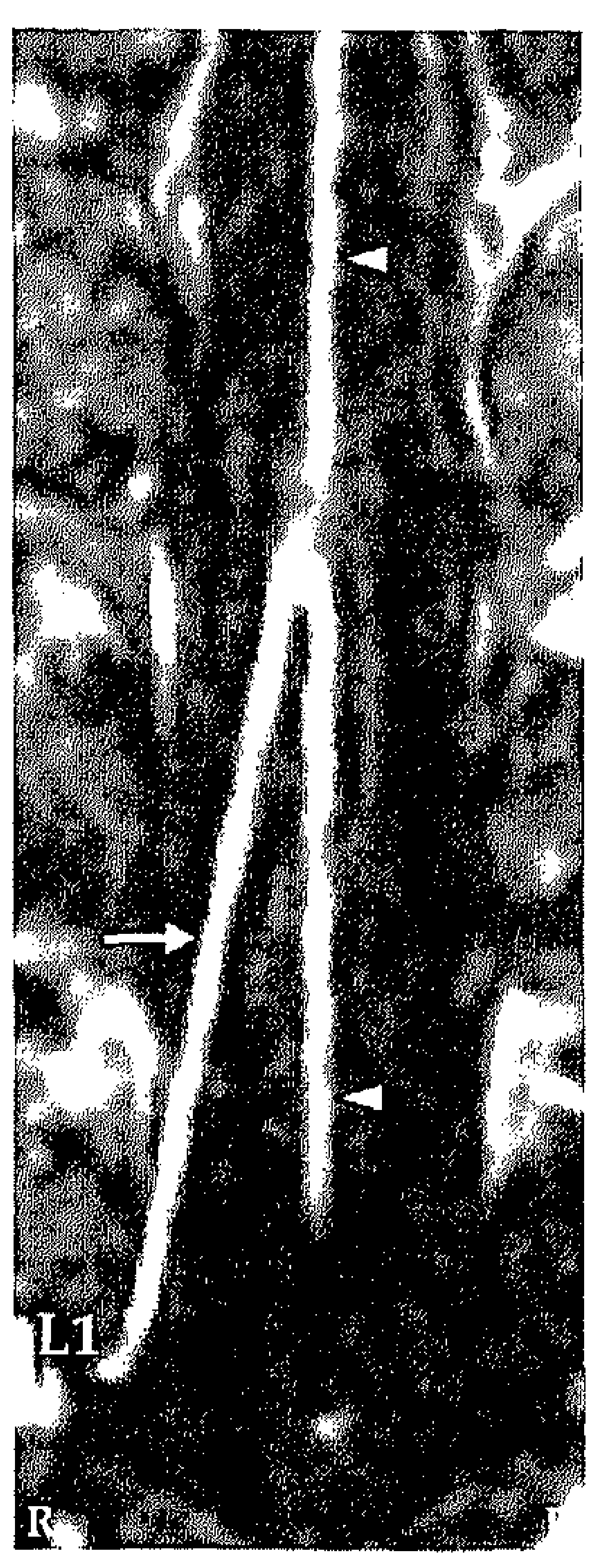

a
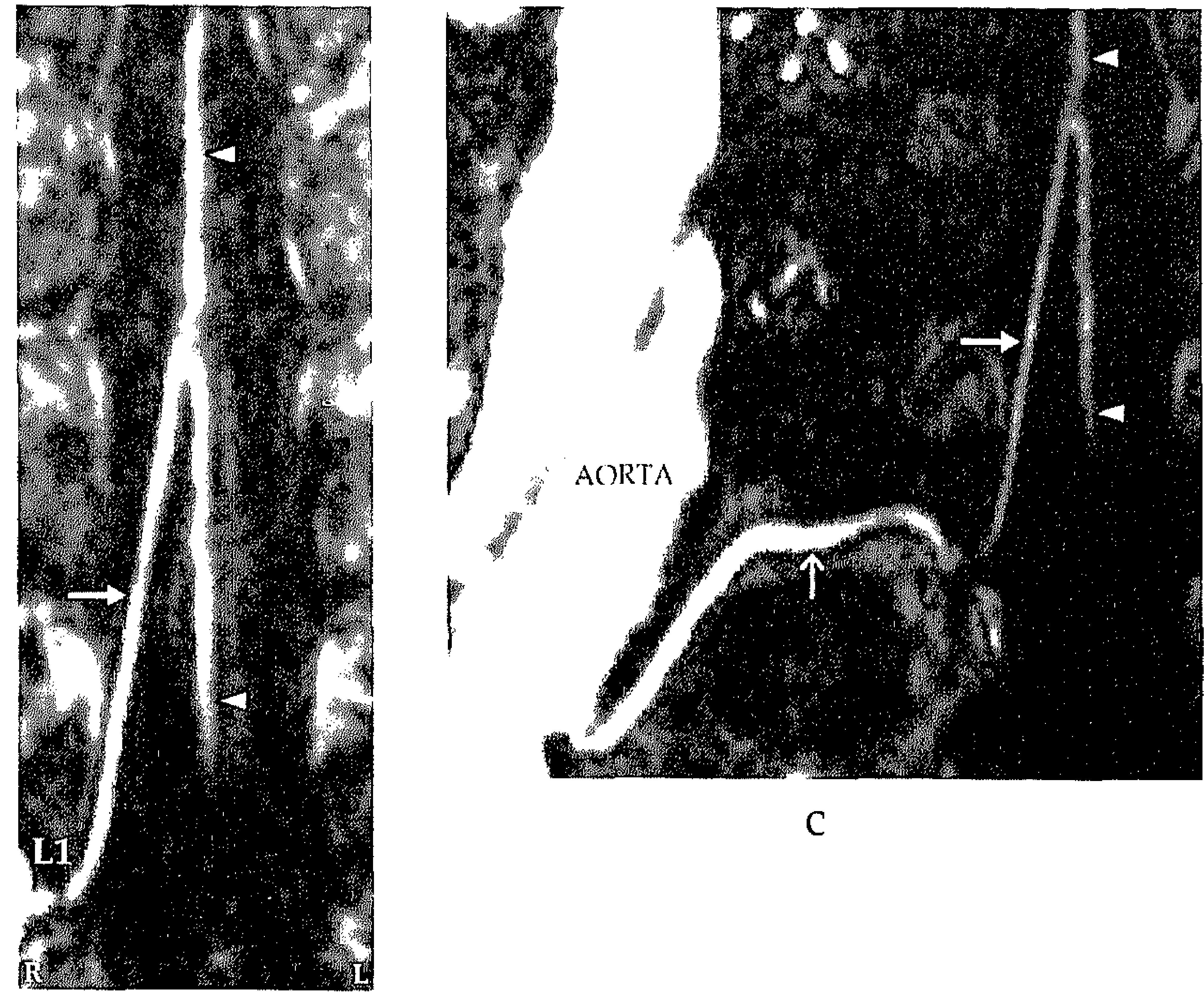

C

$\mathrm{b}$

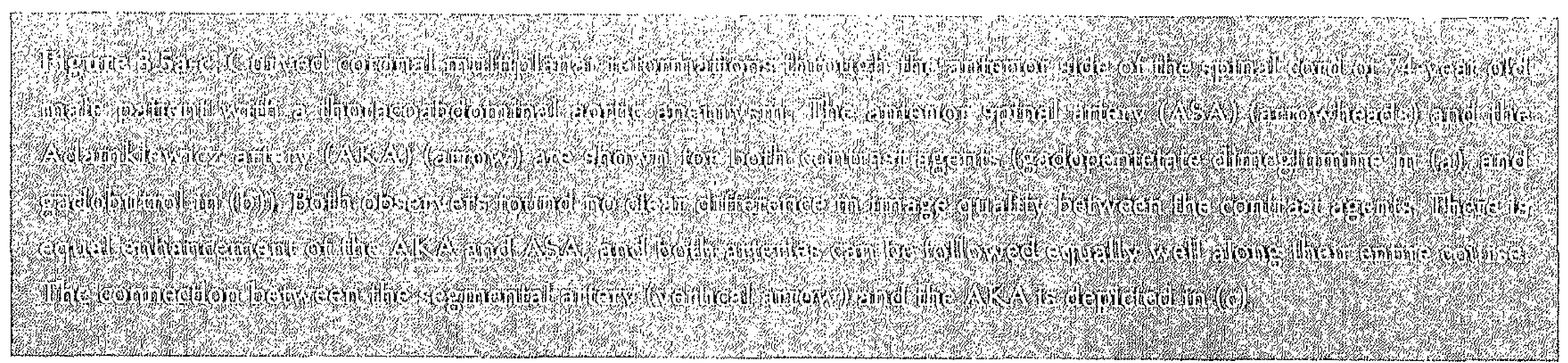

the way from L5 up to T5 in all cases, particularly for the high and middle part of the thoracic segments. It is in this part of the spine where the ASA has its smallest diameter. Previously, we have demonstrated in patients with a thoracic hernia without any vascular abnormalities, that when using the same imaging protocol as described in the current study the depiction of arteries is limited to a diameter above $0.3 \mathrm{~mm}^{9}$. According to the results reported in the vascular anatomic literature 7.8 , the ASA appears to be smaller than $0.3 \mathrm{~mm}$ in the thoracic segments. Another possible reason why depiction of the ASA in the high and middle thoracic segments was not achieved, is that this region suffers the most from image artifacts (streaks) related to cardiac motion. To overcome the cardiac motion artifacts, one might suggest altering the slice orientation from sagittal to coronal. 

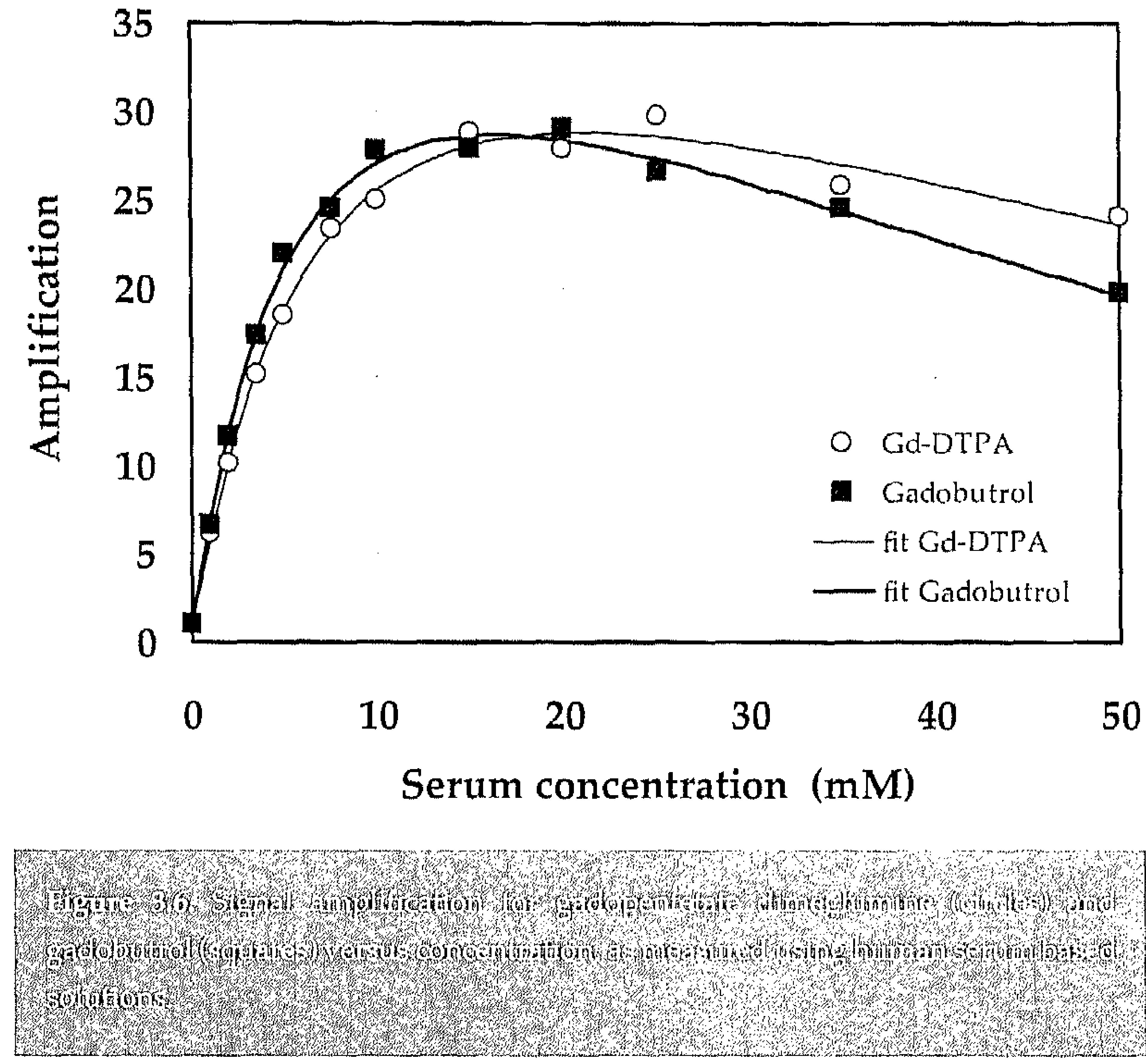

However, when a coronal plane is chosen several problems arise. When the total volume is not within the FOV, foldover artifacts occur in the phase encoding direction. However, we feel that the use of sagittal slices is optimal, for which the phase encoding direction was perpendicular to the coil surface. As such, the FOV in the anterior-posterior (AP) direction could be limited to $35 \%$ to $50 \%$ of the FOV in the feet-head direction and thus reduce imaging time. Due to the use of a phasedarray surface coil, which has a decreasing signal sensitivity in the AP direction, foldover artifacts in the AP direction were absent. Using coronal slice direction, on the contrary, would yield serious foldover artifacts when trying to reduce imaging time by limiting the left-right FOV.
The injection rates of the two contrast agents were chosen to obtain an equal first-pass arterial peak concentration in the aorta. This peak concentration approximately equals the injection rate (in $\mathrm{mmol} / \mathrm{sec}$ ) divided by the cardiac output (assumed to be 5 liters $/ \mathrm{min}$ ). This amounts to an arterial peak concentration of approximately $18 \mathrm{mM}$, which very nicely fits into the concentration range where both contrast agents show their maximum signal amplification.

It is noted that the phantom experiment only provides a model to compare (in vitro) signal enhancement between the two contrast agents, but is not accurate for absolute quantification of in vivo signal enhancement as this is influenced by many other local factors (e.g., magnetic susceptibility and partialvolume effects).

Maximum enhancement of the spinal cord arteries is essential in order to optimally visualize these vessels. Matching the arterial peak contrast concentrations by adjusting the injection rates of both contrast agents, no significant differences in SNR, CNR, and FWHM values were obtained between GdDTPA and Gadobutrol in this study.

One could argue that the number of patients investigated in this study was limited, and that significant differences might appear using a larger sample size. From a clinical point of view, however, we feel that one only decides to change to a new contrast agent when differences are obvious even in a small number of patients.

Comparing the relation between signal enhancement and (arterial) concentration one may observe that Gadobutrol has a higher upslope (i.e., T1 relaxivity) for low concentrations and a stronger $\mathrm{T}^{*}$ decay for high concentrations (Figure 3.6). This means that Gadobutrol 
may be beneficial when used at low arterial concentrations (e.g., slower injection rate), where $\mathrm{T}^{*}$ effects are less important, compared to Gd-DTPA. However, when ultrasmall arteries related to spinal cord have to be imaged, higher arterial concentrations are required to achieve the highest signal amplification and $\mathrm{T} 2 *$ signal reducing effects become relevant.

Previous studies indicated that a high dose $(>0.2 \mathrm{mmol} / \mathrm{kg}$ ) of Gd-DTPA is advised to depict the ultrasmall arterial trajectories to the spinal cord $3-6,9$. The use of a triple dose $(0.3$ $\mathrm{mmol} / \mathrm{kg}$ ) of contrast agent has been reported to be safe for both contrast agents 12,13 . In this study, maximum signal amplification, however, turned out to be equal for the two contrast agents. An argument that could favor Gadobutrol above Gd-DTPA is the notion that although during the trailing flank of the dynamic contrast bolus the signal amplification is lower than its maximum value; it is higher for Gadobutrol than for Gd-DTPA. In image acquisition terms the trailing flank of the bolus corresponds to peripheral k-space profiles, which are responsible for image sharpness. In addition, as the average bolus arrival time is later for Gadobutrol, due to the slower injection, one might expect a longer contrast bolus which again should emphasize the peripheral k-space profiles compared to GdDTPA. However, no significant differences in image sharpness were observed between the two agents. It is furthermore noted that there is quite some uncertainty about which concentration is actually achieved in TAAA patients due to inter- and intraindividual variations in cardiac output, and because it is unknown if and how contrast kinetics are altered in the presence of an aortic aneurysm. A broader maximum in the signal amplification as was found for Gd-DTPA is less sensitive to such concentration uncertainties than a sharper maximum as for Gadobutrol.

Our results are in contrast with the results found in other comparative studies by Goyen et al ${ }^{10,11}$, who used a different pulse sequence. They found that the use of Gadobutrol increased the SNR and CNR by $70 \%$ compared to Gd-DTPA. Vessel delineation was furthermore reported to improve, particularly for small vessels. Although the improvement in vessel delineation was found to be not statistically significant, it was pointed out that the use of Gadobutrol may be beneficial for small vessel delineation in three-dimensional MR angiography. Goyen et al ${ }^{10,11}$ studied the relatively large arteries branching directly from the aorta (iliac arteries, carotid arteries, and renal arteries), which had a relatively high flow. Using Gadobutrol, they observed more arteries deriving from the common iliac arteries compared to Gd-DTPA. Other important differences between the study by Goyen et al 10,11 and the current study are differences in injection rate and the use of a shorter echo time. The study of Goyen et al ${ }^{10,11}$ used an injection rate of $0.5-0.8 \mathrm{mmol} / \mathrm{sec}$, which is two to three times slower than the injection rate (1.5 $\mathrm{mmol} / \mathrm{sec}$ ) of this study. Assuming an equal cardiac output, this means that a two to three times lower arterial concentration is achieved, which corresponds to the concentration region where Gadobutrol shows stronger $\mathrm{T} 1$ weighted signal amplification than Gd-DTPA. Goyen et al10,11 have used in both their comparison studies a lower echo time $\left(0.7 \mathrm{msec}^{11}\right.$ and 0.88 msec $^{10}$ vs. $1.9 \mathrm{msec}$ in this study). Using a shorter TE is beneficial as it decreases the unavoidable T2* signal decay of Gadobutrol compared to Gd-DTPA. Looking at the intrinsic properties of both agents, Gadobutrol would 
benefit the most from TE shortening; T1 and $\mathrm{T}^{*}$ relaxivities are both higher compared to Gd-DTPA. Shortest echo time is determined by several scan parameters. The two most important ones encountered in spinal cord MR angiography are the FOV (in the frequency encoding direction) and the matrix dimension. To image the arterial pathway to the spinal cord, a large volume with high spatial resolution (i.e., large image matrix) has to be employed, which inherently increases the minimum echo time. The current shortest possible TE of $1.9 \mathrm{msec}$ can only be lowered by using stronger magnet gradients.

Another recent study by Fink et a ${ }^{14}$ found that the use of Gadobutrol over Gd-DTPA is not advantageous for pulmonary perfusion imaging. In their study, Fink et al ${ }^{14}$ used a high injection rate $(5 \mathrm{~mL} / \mathrm{sec})$ and a lower dose of contrast agent for both agents. The dose for Gadobutrol differed between 0.1 and 0.2 $\mathrm{mmol} / \mathrm{kg}$. For Gd-DTPA, a maximum dose of $0.2 \mathrm{mmol} / \mathrm{kg}$ was given. They hypothesized that the $\mathrm{T} 2^{*}$ signal decay was the reason why no advantage was found. As was found in the current study, the high injection rate leads to a high arterial concentration, where there is little difference between the two contrast agents in signal amplification. For high concentrations the signal enhancement decreases, an effect which is stronger for Gadobutrol than for GdDTPA because of the stronger $\mathrm{T}_{2}$ effects. In contrast to T1-weighted imaging, the increasing T2* effects of Gadobutrol at higher concentrations turn out to be beneficial when used for $\mathrm{T} 2{ }^{*}$-weighted imaging. Tombach et $\mathrm{al}^{15}$ for instance, demonstrated for brain perfusion studies that a triple dose of $1.0-\mathrm{M}$ Gadobutrol yields stronger changes in the time course of the $\mathrm{T}^{*}$ relaxation rates compared to 0.5-M Gadobutrol.
Although we reported that we have visualized the supplying arteries, one could argue that by using this MR technique venous enhancement is likely to appear, as relatively long acquisition times are used. The MR angiography acquisition is performed using elliptical centric k-space ordering to suppress venous enhancement. In the first 4 to 8 seconds of this acquisition, central k-space profiles, which determine contrast, are acquired. In this initial phase, the arteries will contain most of the contrast agent and thereby will consequently enhance more strongly, as the arteriovenous window in the spinal cord circulation is approximately 10 seconds $^{16}$. Regarding the configuration of the intradural arteries and veins, there is much resemblance. Therefore, at present the only reliable discrimination between intradural arteries and veins can be made by using catheter angiography. In this study no catheter angiography was performed; therefore, we cannot rule out some contribution of venous enhancement for the intradural arteries.

In conclusion, we have demonstrated that for imaging the arteries supplying the spinal cord in TAAA patients, both Gadobutrol and Gd-DTPA are equally well suited. Using both contrast agents it was possible to consistently visualize the ultrasmall arteries supplying the spinal cord in $100 \%$ of cases. It was moreover demonstrated by using two contrast agents, that the applied MR angiography technique is capable of reproducibly and consistently depicting the ultrasmall blood supplying arteries (ASA and AKA) of the spinal cord.

At this moment, Gadobutrol is more expensive than Gd-DTPA. Considering this and our results, showing no significant image quality differences, we find no benefit in using Gadobutrol in high arterial concentration MR angiography for arteries supplying the spinal cord. 


\section{REFERENCES}

1. Williams GM, Roseborough GS, Webb TH, Perler BA, Krosnick $T$. Preoperative selective intercostal angiography in patients undergoing thoracoabdominal aneurysm repair. I Vasc Surg 2004;39:314-21.

2. Kieffer E, Fukui S, Chiras J, Koskas F, Bahnini A, Cormier E. Spinal cord arteriography: a safe adjunct before descending thoracic or thoracoabdominal aortic aneurysmectomy. I Vasc Surg 2002;35:262-8.

3. Yoshioka K, Niinuma $H$, Ohira A, et al. MR angiography and $C T$ angiography of the artery of Adamkicwicz: noninvasive preoperative assessment of thoracoabdominal aortic aneurysm. Radiographics 2003;23:1215-25.

4. Yamada N, Okita Y, Minatoya K, et al. Preoperative demonstration of the Adamkiewicz artery by magnetic resonance angiography in patients with descending or thoracoabdominal aortic aneurysms. Eur J Cartiothornc Surg 2000;18:104-11.

5. Yamada N, Takamiya M, Kuribayashi S, Okita $Y$, Minatoya K, Tanaka R. MRA of the Adankiewicz artery: a preoperative study for thoracic aortic aneurysm. / Comput Assist Tomogr 2000;24:362-8.

6. Kawaharada N, Morishita $\mathrm{K}$, Hyodoh $\mathrm{H}$, et al. Magnetic resonance angiographic localization of the artery of Adamkiewicz for spinal cord blood supply. Ann Thorac Surg 2004;78:846-51; discussion 51-2.

7. Koshino T, Murakami G, Morishita K, Mawatari T, Abe $T$. Does the Adamkiewicz artery originate from the larger segmental arteries? I Thorac Cardiovnsc Surg 1999;117:898-905.

8. Thron A. Anatomy of the spinal cord's blood supply. In: Thron A, ed. Vascular anatomy of the spinal cord. Wien: Springer-Verlag; 1988:8-12.

9. Nijenhuis RJ, Leiner T, Cornips EM, et al. Spinal cord feeding arteries at MR angiography for thoracoscopic spinal surgery: feasibility study and implications for surgical approach. Radiology 2004;233:541-7.
10. Goyen M, Lauenstein TC, Herborn CU, Debatin JF, Bosk S, Ruehm SG. 0.5 M Gd chelate (Magnevist) versus 1.0 M Gd chelate (Gadovist): doseindependent effect on image quality of pelvic threedimensional MR-angiography. I Magn Reson lmaging 2001;14:602-7.

11. Goyen $\mathrm{M}$, Herborn CU, Vogt FM, et al. Using a $1 \mathrm{M}$ Gd-chelate (gadobutrol) for total-body threedimensional MR angiography: preliminary experience. J Magn Reson Imaging 2003;17:565-71.

12. Cochran ST, Bomyea K, Sayre JW. Trends in adverse events after IV administration of contrast media. $A J R$ Am J Roentgenol 2001;176:1385-8.

13. Balzer JO, Loewe C, Davis K, et al. Safety of contrastenhanced MR angiography employing gadobutrol $1.0 \mathrm{M}$ as contrast material. Eur Radiol 2003;13:2067-74.

14. Fink C, Puderbach M, Ley S, ct al. Contrast-enhanced three-dimensional pulmonary perfusion magnetic resonance imaging: intraindividual comparison of 1.0 $\mathrm{M}$ gadobutrol and $0.5 \mathrm{M}$ Gd-DTPA at three dose levels. Invest Radiol 2004;39:143-8.

15. Tombach $B$, Benner $T$, Reimer $P$, et al. Do highly concentrated gadolinium chelates improve MR brain perfusion imaging? Intraindividually controlled randomized crossover concentration comparison study of 0.5 versus $1.0 \mathrm{~mol} / \mathrm{L}$ gadobutrol. Radiology 2003;226:880-8.

16. Pattany PM, Saraf-Lavi E, Bowen BC. MR angiography of the spine and spinal cord. Top Mngn Reson Imaging 2003;14:444-60. 


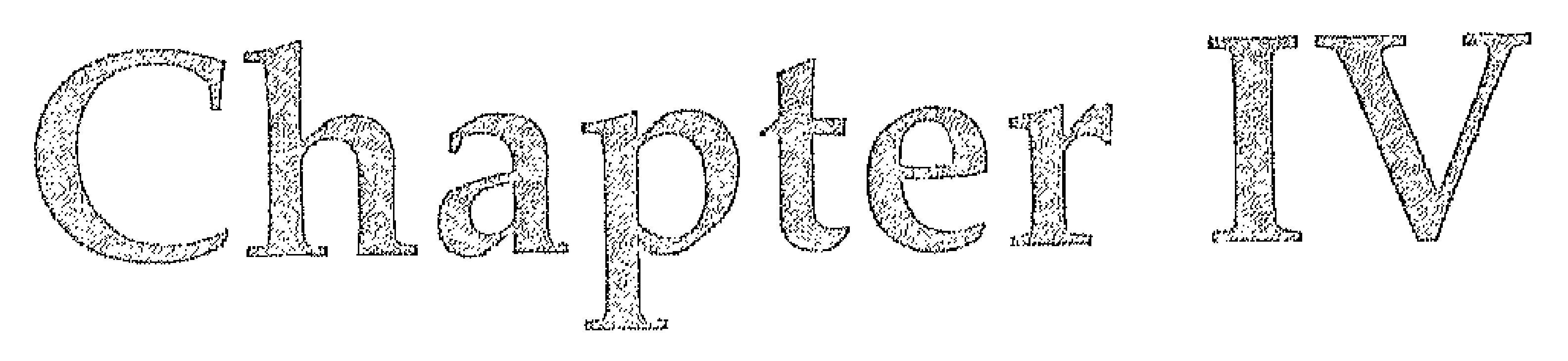

\section{Differentiation of spinal cord arteries and veins by time-resolved MR angiography}




\begin{abstract}
Purpose: To dynamically resolve the inlet arteries and outlet veins of the spinal cord, particularly the Adamkiewicz artery (AKA) and great anterior radiculomedullary vein (GARV), using magnetic resonance (MR) angiography.

Materials and Methods: First, conventional two-phase angiography (acquisition time 38-55 seconds) utilizing elliptic centric $k$-space ordering was applied to aortic aneurysm patients. Changes of vessel intensity were compared between two subsequent dynamic phases. Computer modelling of bolus enhancement and k-space sampling was performed to demonstrate the relation between vessel enhancement, acquisition time, and vessel diameter. Second, timeresolved (keyhole) angiography using reduced number of phase encoding steps was explored in healthy volunteers and aortic aneurysm patients using acquisition times (range 6-8.5 seconds) shorter than the spinal cord circulation time.
\end{abstract}

Results: Using two-phase angiography the AKA and GARV were co-visualized in the early phase, and contrast decreased for the AKA and increased for the GARV in most (70\%) but not all cases. Computer modeling showed that the arteriovenous contrast strongly depended on vessel diameter and complete separation was only obtained with short acquisition times. Using time-resolved angiography complete temporal separation of the AKA and GARV was realized in all cases $(100 \%)$.

Conclusion: The AKA and GARV can be completely separated by time-resolved MR angiography. 


\section{INTRODUCTION}

Separation of arteries and veins is a general problem and challenge in non-invasive vascular radiology. This differentiation is especially difficult for the anterior spinal cord vasculature, since the vessels have a small caliber $(0.2-1.5 \mathrm{~mm})$, a close anatomic relation, and a more or less similar spatial configuration ${ }^{1}$. Therefore, distinction solely based on vascular anatomic features is unreliable. Selective identification and localization of the inlet arteries of the thoracolumbar spinal cord, in particular the great anterior radiculomedullary artery, i.e. the Adamkiewicz artery (AKA), is of relevance in several clinical situations. Possible indications for AKA localization include thoracoabdominal aortic aneurysm (TAAA) surgery $2-5$, thoracoscopic spinal discectomy ${ }^{6}$, and embolization of vertebral metastases ${ }^{7}$ and spinal dural arteriovenous malformations ${ }^{8}$.

The standard of reference for spinal cord vascular imaging is intra-arterial catheter $(X$ ray) angiography, also known as digital subtraction angiography, which enables selective visualization of arteries and veins. Despite the superior image quality of spinal cord catheter angiography, it carries risk for neurologic complications and can only be performed at highly specialized centers. Moreover, it has a limited and variable sensitivity $(43-86 \%)^{9,10}$ for identifying the AKA in TAAA patients. Recently promising results have been achieved in non-invasive visualization of the spinal cord vasculature in patients with TAAA and spinal cord vascular abnormalities, by employing magnetic resonance (MR) angiography $3,4,11-14$.

Because MR acquisition times tend to be long relative to catheter angiography, advanced acquisition techniques like elliptic centric k-space filling are used to suppress venous signal15-17. Even though this technique is successful for suppressing for instance segmental veins relative to segmental arteries ${ }^{6}$, separate visualization of the AKA from the great anterior radiculomedullary vein (GARV) is generally not achieved. This is probably related to the fact that the acquisition times (18-45 seconds) are quite longer than the spinal circulation time of approximately 10 seconds ${ }^{18}$, resulting in simultaneous enhancement of both the arteries and veins.

Ideally, arteriovenous differentiation should be based on differences in contrast agent arrival time, analogous to catheter cine angiography. To achieve sufficiently high temporal resolution MR angiography has acquisition times can be shortened by reducing the number of phase encoding steps, for example by limiting the spatial coverage or by applying keyhole imaging 19-21. To our knowledge this potential of MR angiography has not been explored for spinal cord angiography.

The aim of this study was two-fold: (i) to quantify signal enhancement of the AKA and GARV using a two-phase conventional elliptic centric k-space ordered MR angiography technique in TAAA patients and to elucidate the principles why the relatively thick segmental arteries and veins are completely separated, but thin spinal cord vessels are not, using a computer model of elliptic centric kspace sampling, and (ii) to investigate the possibilities to dynamically and completely resolve enhancement of the AKA from the GARV in both healthy volunteers and TAAA patients. 


\section{MATERIALS and METHODS}

All imaging was performed on a clinical 1.5-Tesla MRI system, using a phased-array surface spine coil (Philips Medical Systems, Best, The Netherlands). Subjects were imaged in supine position. All subjects gave informed consent prior to the study, which was approved by the medical ethics committee of the hospital.

\section{Troo-phase $M R$ angiography}

Subjects. Twenty patients (8 men and 12 women) underwent MR angiography prior to elective TAAA repair. The mean age was 66 years (range, 29-80 years).

MR imaging. A T2-weighted fast spin echo acquisition was performed to visualize the anatomy of the spine and localize the vertebrae relative to the embedded coil markers, which served as a reference to determine the level of the segmental artery connecting to the AKA. Acquisition parameters were TR $2686 \mathrm{msec}$; TE $120 \mathrm{msec}$; and flip angle $90^{\circ}$. Craniocaudal field of view (FOV) was $480 \mathrm{~mm}$ and the number of sagittal slices was 11 . Measured voxel size was $1.25 \mathrm{x}$ $1.67 \times 4.0 \mathrm{~mm}$.

The scan delay time between contrast medium administration and image acquisition was determined by MR fluoroscopy. Scan parameters were TR $5.2 \mathrm{msec}$; TE $1.4 \mathrm{msec}$; flip angle $35^{\circ}$; FOV $450 \mathrm{~mm}$; and matrix size $205 \mathrm{x}$ 256. A test bolus of $2 \mathrm{~mL}$ gadopentetate dimeglumine (Magnevist, Schering $A G$, Berlin, Germany) was injected at a rate of 3 $\mathrm{mL} / \mathrm{sec}$, followed by a $25 \mathrm{~mL}$ saline flush injected at the same rate. One sagittal slice (thickness, $80 \mathrm{~mm}$ ), placed through the aorta, was acquired each second during two minutes. The scan delay time was defined as the time between the start of contrast agent administration and earliest enhancement of the entire abdominal aorta.

Subsequently, first-pass three-dimensional MR angiography was performed. To this end, $45 \mathrm{~mL}$ (0.3 mmol per kilogram body weight) of gadopentetate dimeglumine was injected in the antecubital vein of the left arm at a rate of $3 \mathrm{~mL} / \mathrm{sec}$ and flushed with $25 \mathrm{~mL}$ saline at the same rate. The acquisition consisted of two dynamic phases. The first phase was started after the determined scan delay time. Acquisition of the second dynamic phase started immediately after the first was finished. The pulse sequence used was a threedimensional fast-field echo (FFE), with the frequency encoding direction planned craniocaudally. The craniocaudal FOV was $520 \mathrm{~mm}$ and anterior-posterior FOV was 180 $\mathrm{mm}$ (i.e., rectangular field of view (RFOV) $35 \%)$. To cover the entire axial cross-sections of the vertebrae the number of sagittal slices was individually adjusted (range, 84-110). Acquired slice thickness was $1.2 \mathrm{~mm}$ and after reconstruction using $\mathrm{k}$-space zero filling the slice overlap was $0.6 \mathrm{~mm}$. Measured voxel size was $1.1 \times 1.1 \times 1.2 \mathrm{~mm}$. Acquisition parameters were TR 5.4-5.8 msec; TE $1.7 \mathrm{msec}$ (partial echo $62.5 \%$ ); and flip angle $30^{\circ}$. This resulted in acquisition time per phase of 38-55 seconds. $\mathrm{K}$-space was filled using elliptic centric ordering in which the central part of k-space was acquired randomly22 (CENTRA, Philips Medical Systems, Best, The Netherlands).

Image analysis. Curved multiplanar reformation (MPR) (Easy Vision, release 4.0; Philips Medical Systems, Best, the Netherlands) was used to optimally visualize the spinal cord vessels. In the sagittal view, curved paths on the anterior and posterior surface of the spinal cord were drawn. 
Reformation resulted in a curved coronal plane following the midline vessel on the spinal cord surface. Branches of the midline vasculature were optimally visualized over their entire length by locally adjusting the path. The distinction between AKA and GARV was based on vascular morphologic and anatomic differences. Usually, the GARV has a larger caliber, a longer trajectory (i.e., more vertebral levels) from the anterior cord surface to the neuronal foramen, and is located more caudally than the AKA. The AKA was identified as a hairpin shaped vessel ascending from neural foramen to the anterior cord surface for which a connection with a segmental artery could be identified. The level of origin of the AKA was defined as the vertebral level along which the segmental artery was running. The locations of the AKA and the GARV and/or great posterior radiculomedullary vein (GPRV) were jointly determined by two observers.

The signal intensity (SI) was measured in first and second phase images in the following arteries: AKA, segmental artery supplying the AKA, and aorta. The following veins were analyzed: GARV and GPRV and posterior median vein (PMV). The SI of the anterior midline vasculature (AMLV), which was generally the spatially unresolved combination of the anterior spinal artery (ASA) and the anterior median vein (AMV), was measured both at the level of the AKA and at the level of the GARV. Measurement of SI of the segmental vein in the first phase was not possible in most patients and changes thereof were not further quantified. SI was determined on first and second phase images by placing equally sized user-defined regions of interest (ROI) in the vessel lumen. The standard deviation (SD) of the SI in the erector spinae muscle at the same level as the AKA was measured in both phases. The smallest SD was considered to be the best estimate of the noise level. Contrast-to-noise ratio (CNR) of the spinal cord vessels was calculated by dividing the difference in SI between vessel and immediate surrounding tissue by the noise level.

Statistical analysis. For every patient the signal intensity change $(\Delta S \mathrm{SI})$ was calculated. With a (two-tailed) paired Student's t-test the null hypothesis, $\Delta S I=0$, was tested. For $P<0.05 \quad \Delta S I$ was considered statistically significant. The significance of a (Pearson) correlation between the signal change in the AKA and GARV on the one hand and acquisition time and the bolus arrival time on the other hand was tested. The correlation was considered significant for $P<0.0 \dot{5}$. Temporal arteriovenous differentiation was considered successful when $\Delta S I<0$ for the AKA and $\Delta S I$ $>0$ for the GARV. In case no GARV could be detected the SI of the GPRV was used.

\section{Computer model}

Vessel configurations. The 1D k-space model for contrast-enhanced MRI acquisition previously described by Maki et al ${ }^{15}$ was extended to a 2D model. For in vivo MR angiography the read-out direction $\left(k_{z}\right)$ was chosen along the craniocaudal axis, thus matching the longitudinal direction of the spinal cord vessels of interest. The echo sampling in the read-out direction was considered too fast to be influenced by temporal characteristics of the contrast bolus, and therefore assumed to be instantaneous in the computer model. The two phase encoding directions $\left(k_{x^{\prime}}, k_{y}\right)$ were subject to the temporal effects of the relatively slow $k$-space sampling of the contrast bolus and corres- 


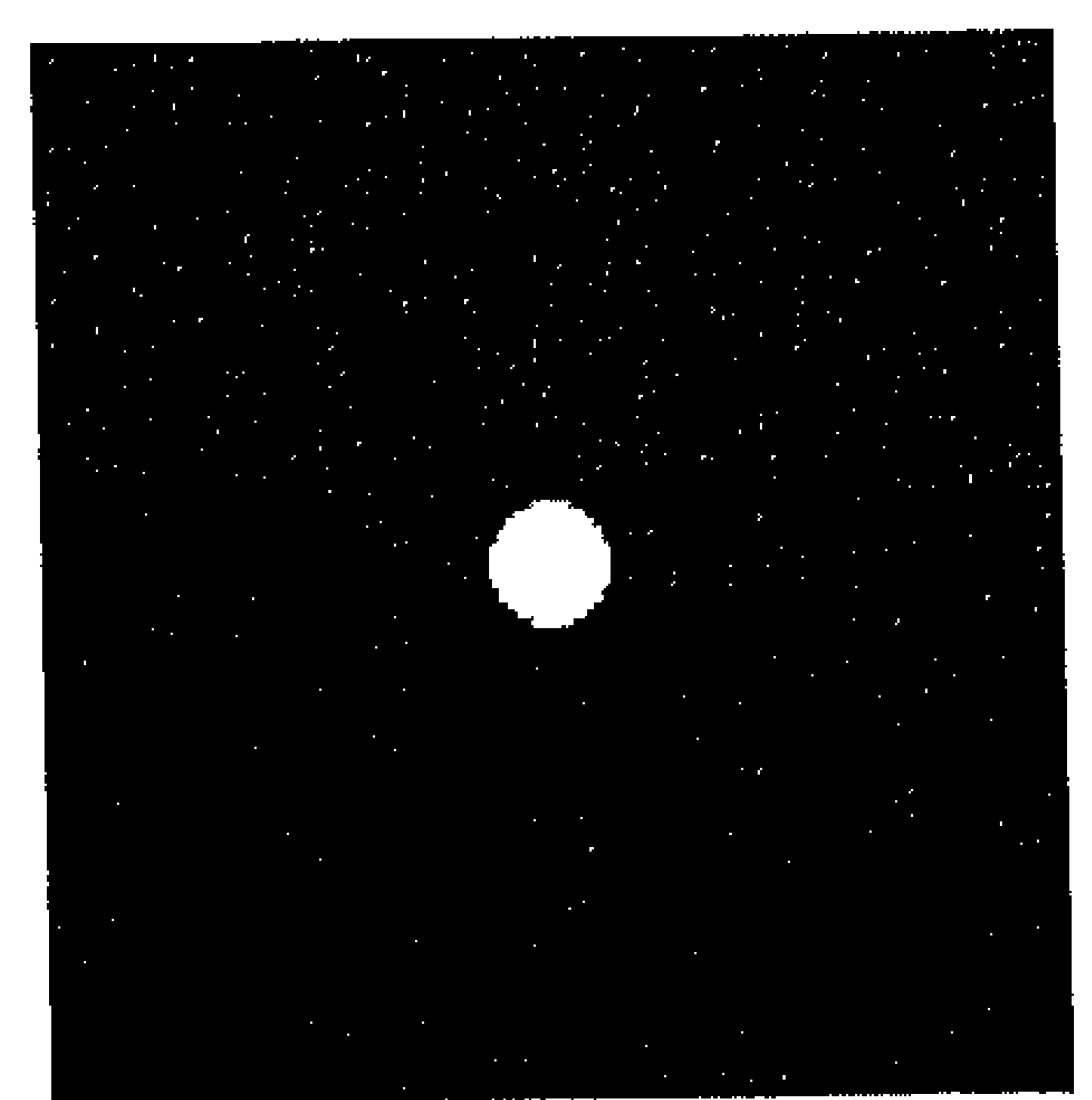

a

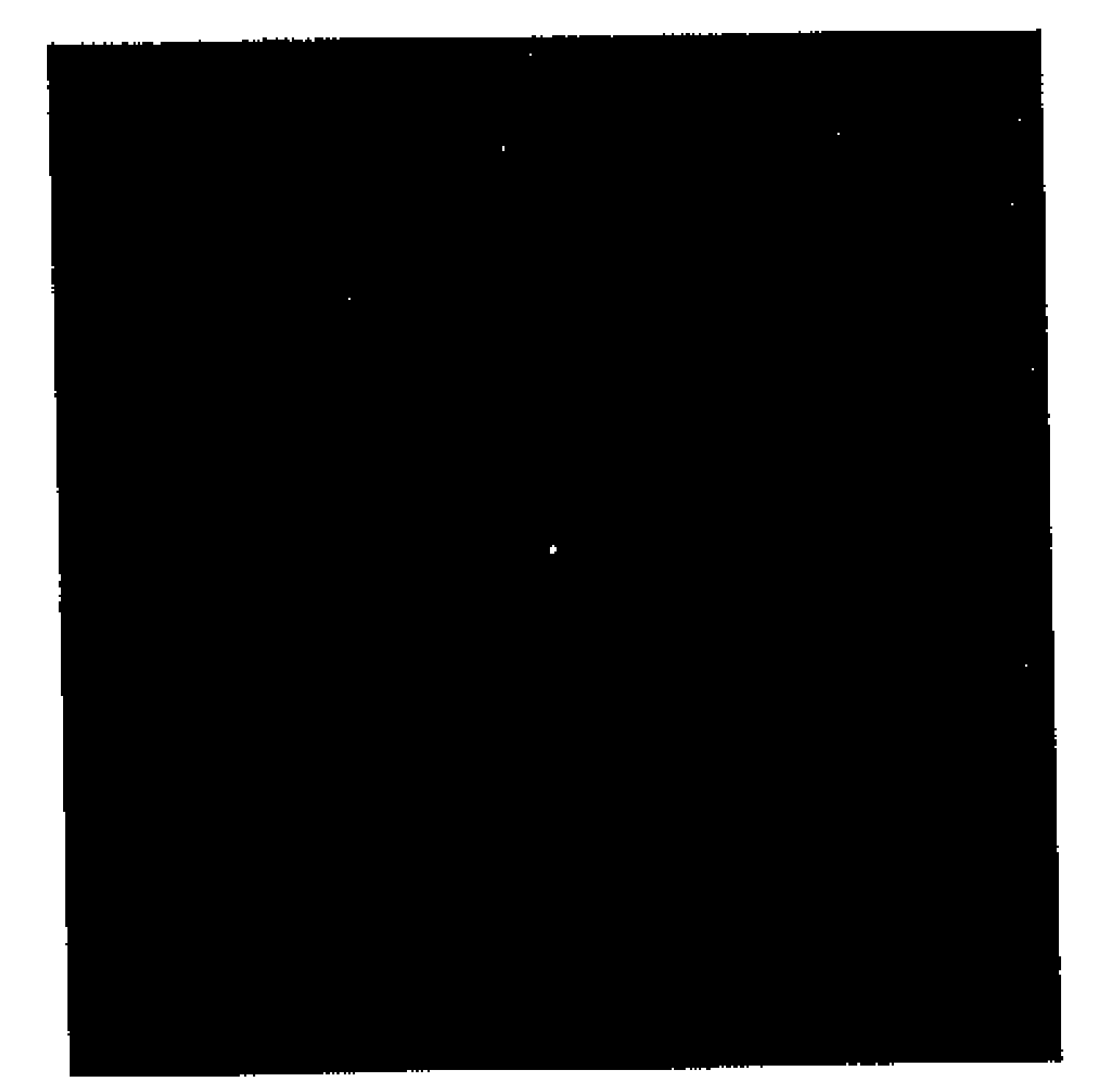

C

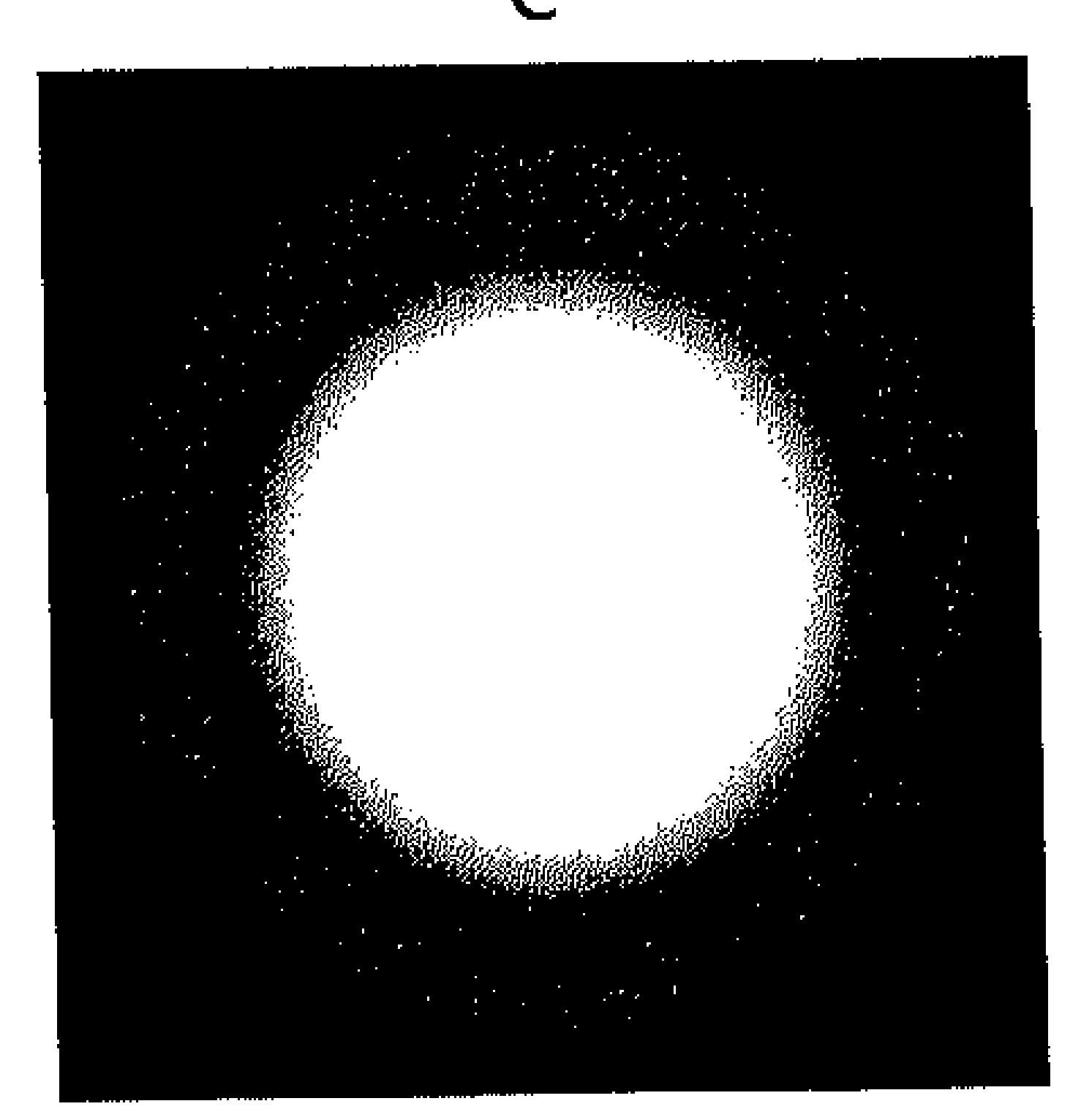

e

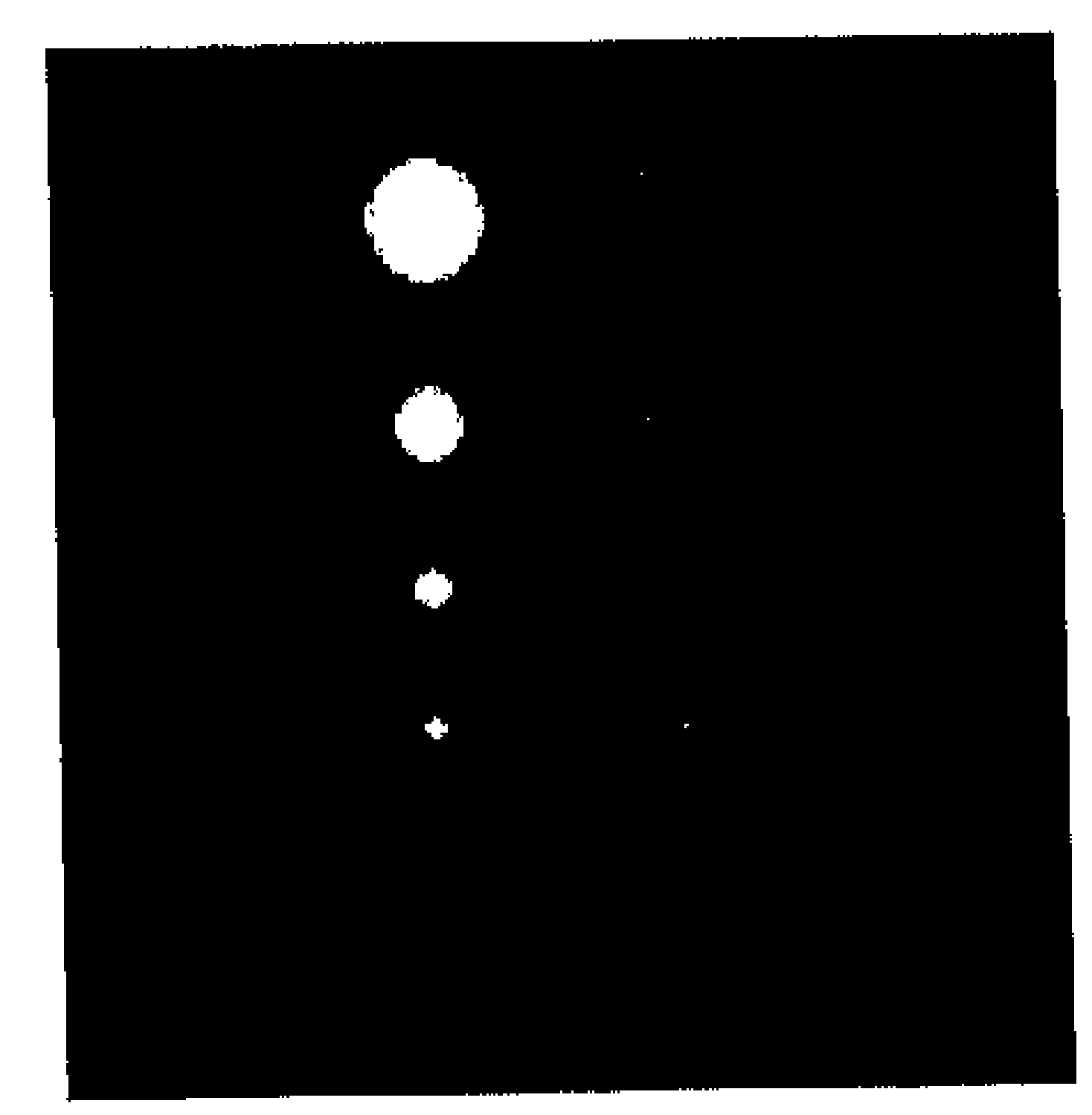

g

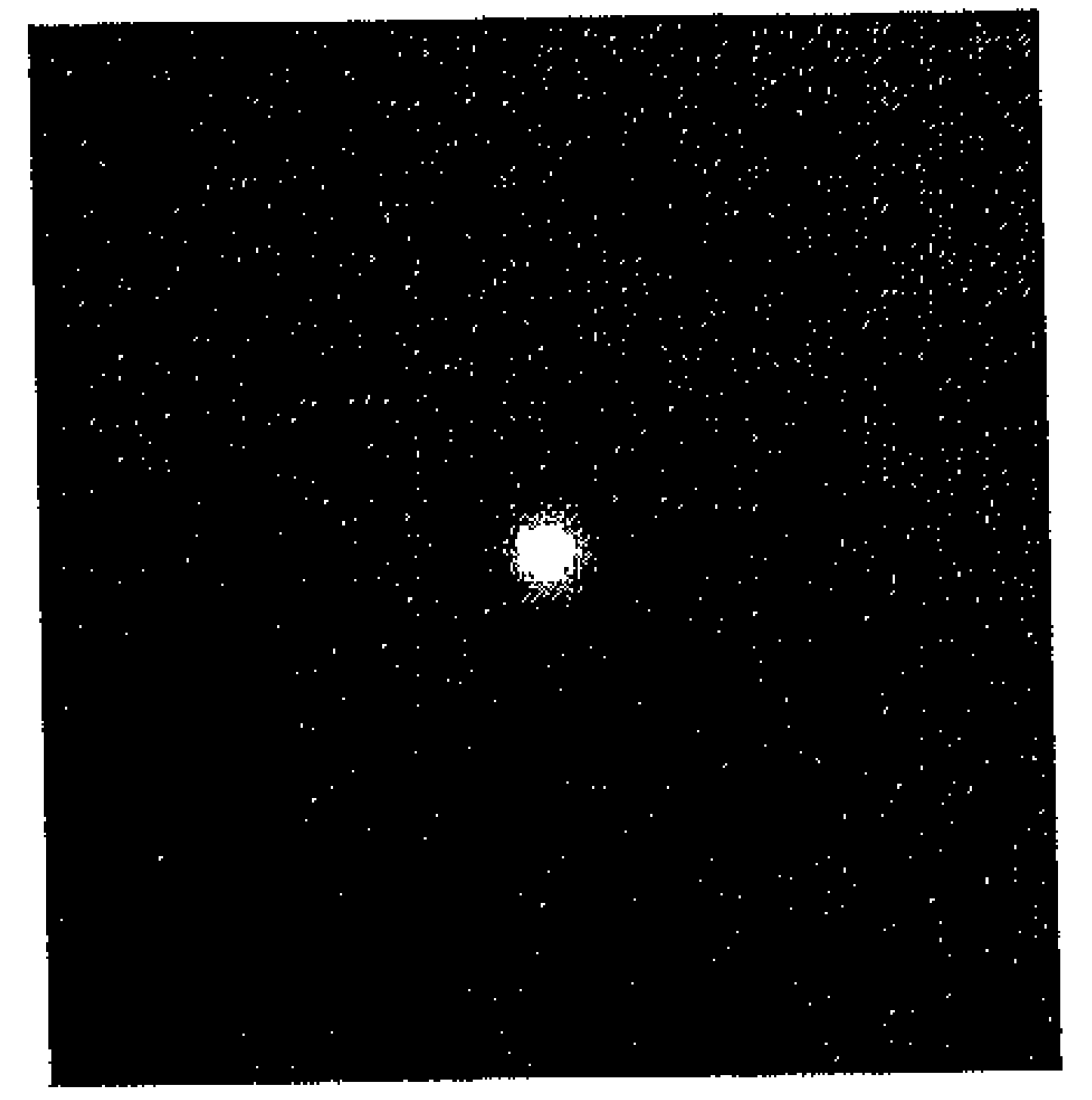

b

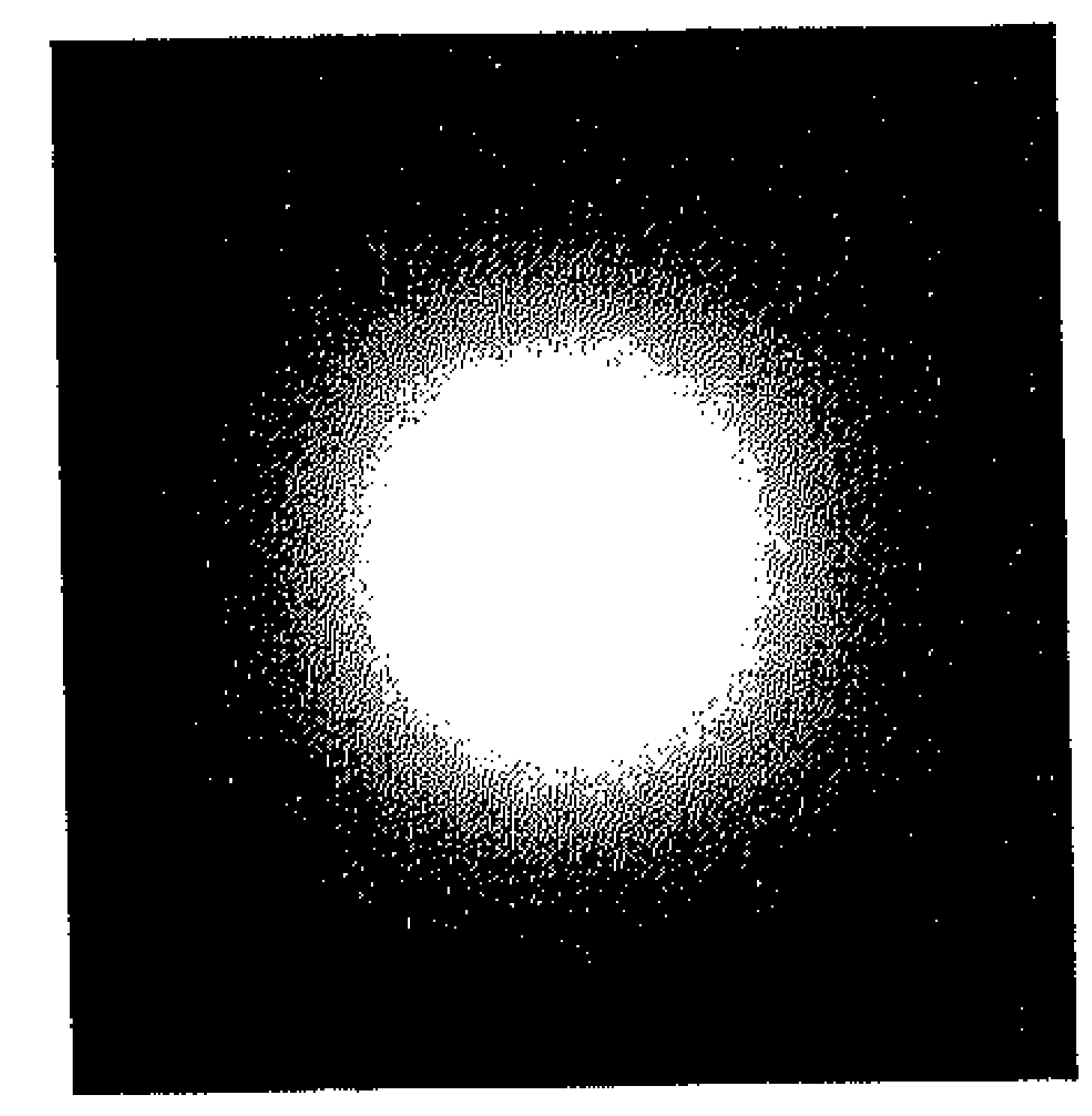

d

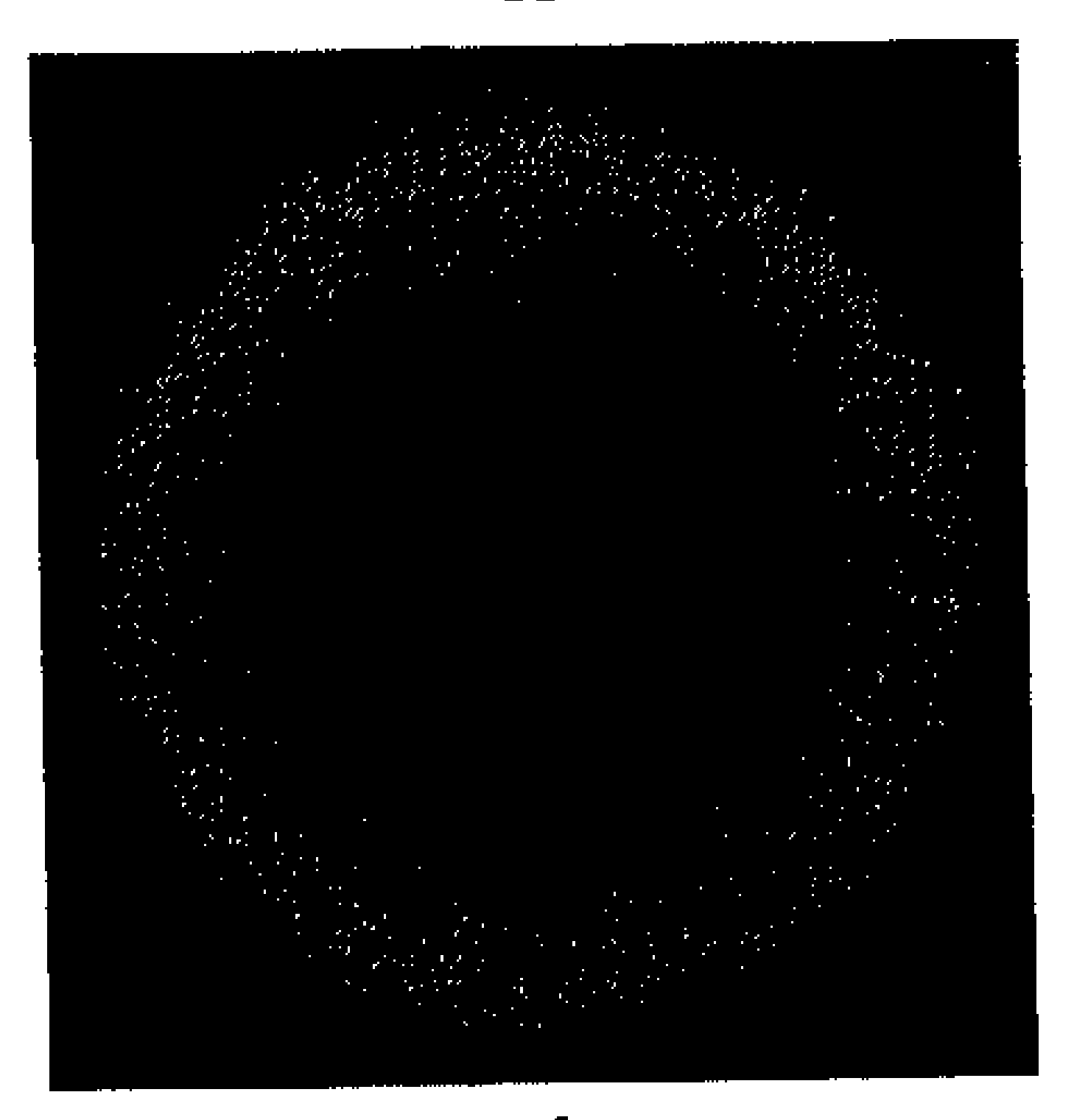

f

ponded to the plane $(x, y)$ of the axial crosssection of the vessels in the computer model. The model incorporated vessels of varying diameters and non-enhancing background tissue. The cross-section of the vessel lumen was modelled by a circular region of pixels (Figure 4.1a and c). Intensities of pixels completely located inside or outside the circular region were set to 0 and 1 , respectively. To pixels partially lying in the circular region, a value corresponding to their fraction inside the circle was attributed. For simplicity reasons, vessel centers were always positioned in the middle of a pixel. The matrix dimension used for the simulations was $128 \mathrm{x}$ 128 pixels. Vessel diameters were expressed in number of pixels rather than in millimeters, since the model turned out to be highly independent of pixel size.

Acquisition simulation. The temporal effect of the contrast bolus passage on k-space sampling was simulated by multiplying the Fourier transforms of the slices depicting the axial vessel cross-sections (Figures $4.1 \mathrm{~b}$ and $\mathrm{d}$ ) with a k-space filter of which each discrete kvalue corresponded with the signal intensity amplification at the time the k-value was acquired (Figure 4.1e and $\mathrm{f}$ for arteries and veins, respectively). K-space sampling along

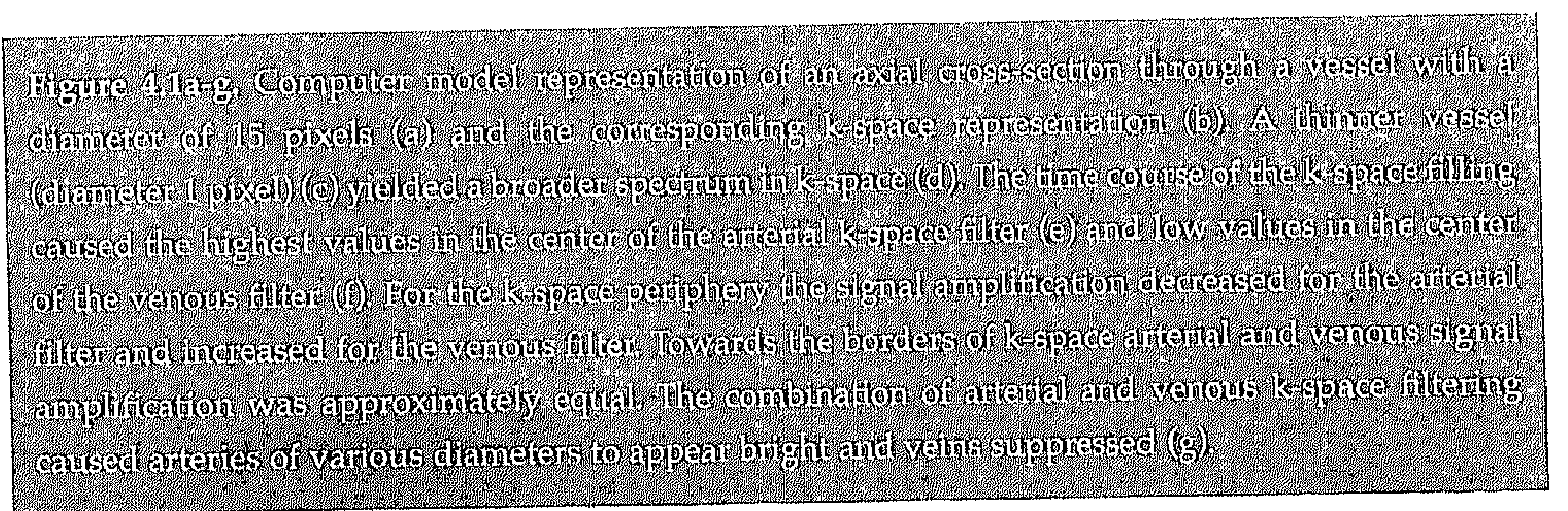


Differentiation of spinal cord arteries and veins

the two phase encoding directions strongly depended on the temporal characteristics of bolus profile and was different between the arterial and venous bolus. The signal amplification time course was measured in the abdominal aorta and inferior vena cava in a TAAA patient after injection of $45 \mathrm{~mL}$ gadopentetate dimeglumine (Magnevist, Schering $A G$, Berlin, Germany) at $3 \mathrm{~mL} / \mathrm{sec}$ to model both arteries and veins (Figure 4.2). Signal amplification was defined as MR signal intensity normalized to the pre-contrast signal intensity.

Simulations and measurements. Vessel diameter varied from 1 to 15 pixels and acquisition times ranging from 10 to 70 seconds were modeled. The image used in further analyses was obtained by calculating the modulus image of the inverse Fourier transform (Figure 4.1g). Relative signal intensity was defined as the mean signal within the borders of the originally defined circular region, normalized to the mean arterial signal obtained for an acquisition time of 10 seconds.

\section{Time-resolved $M R$ angiography}

Subjects. Five healthy volunteers ( 3 men, 2 women; mean age, 26 years; range, 21-39 years) and six TAAA patients (5 men, 1 women; mean age, 65 years; range, 45-75 years) underwent the time-resolved MR angiography exam. Three patients underwent both the conventional and time-resolved $M R$ angiography exam on separate days.

Anatomy and bolus timing. Anatomic images and bolus timing scans were acquired in a similar manner as for the conventional two-phase MR angiography.

Angiography. For time-resolved MR angiography, a three-dimensional FFE techni-

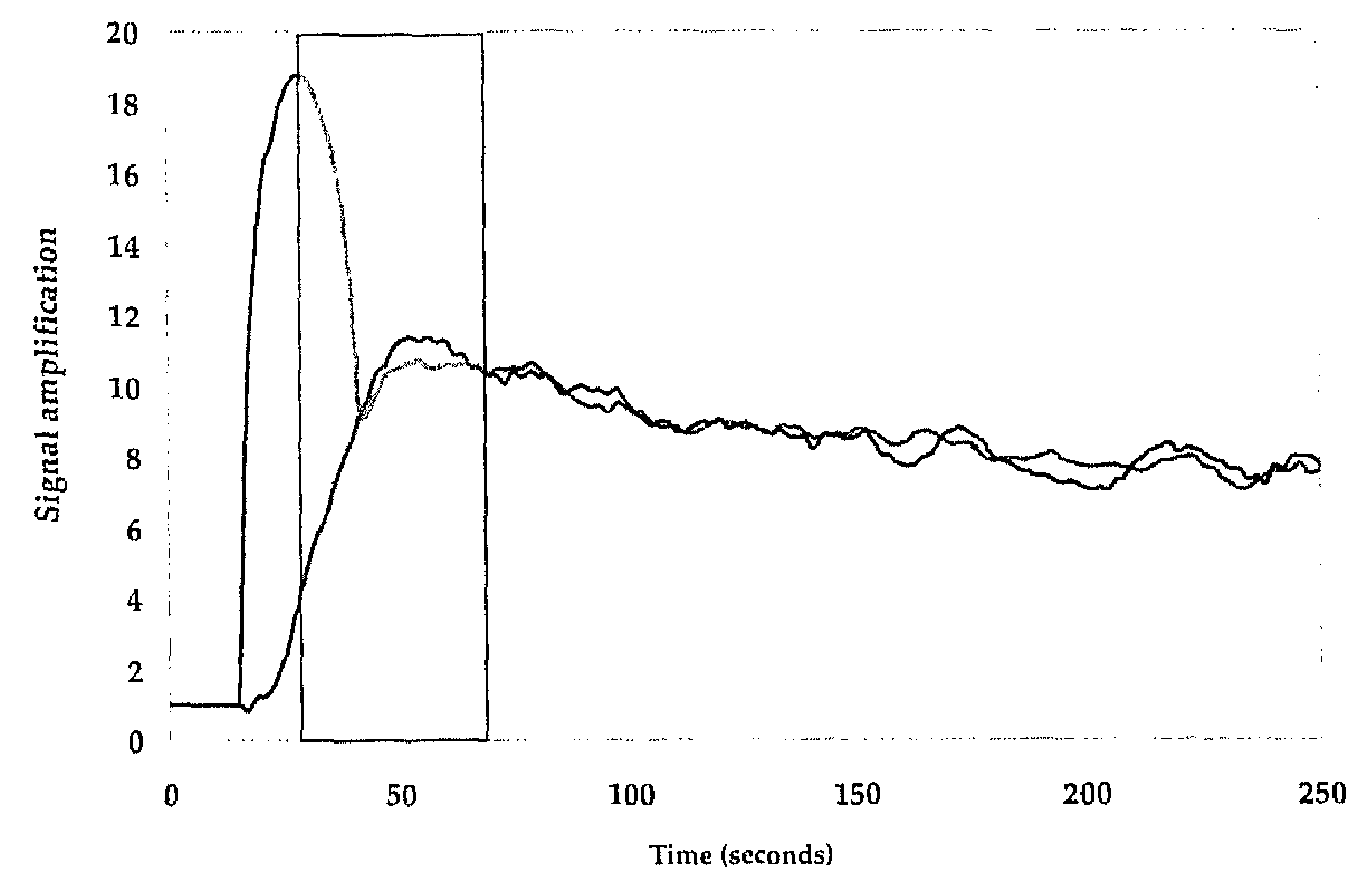

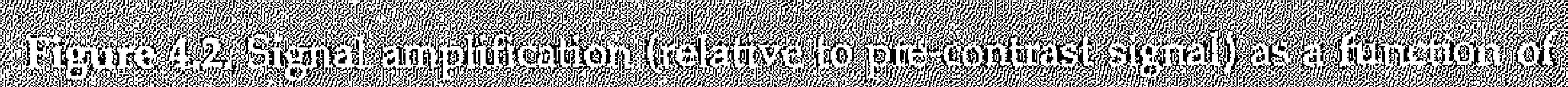

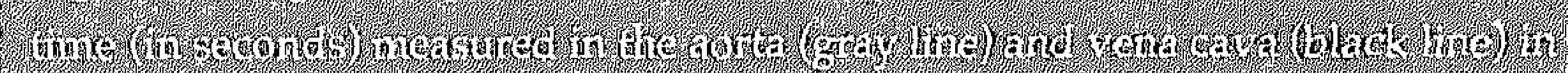

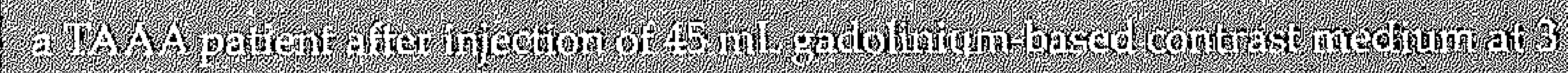

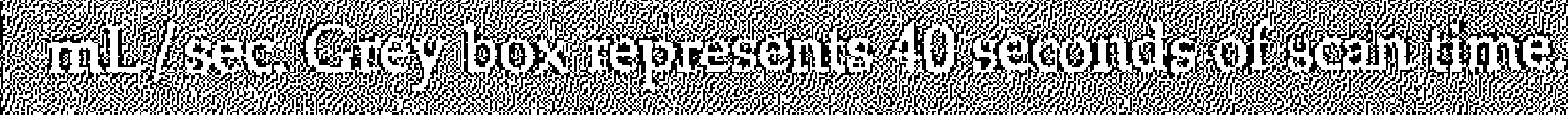

que was used with the following parameters: TR $5.2 \mathrm{msec}$; TE $1.7 \mathrm{msec}$ (partial echo 62.5\%); and flip angle $30^{\circ}$. The scan percentage was set to $100 \%$ and FOV in the craniocaudal frequency encoding direction was $490 \mathrm{~mm}$. The RFOV in the anterior-posterior direction was individually adjusted to prevent foldover of the strongly contrast-enhancing heart and aorta and varied between 30 and $45 \%$ of the craniocaudal FOV. The number of slices (range 30-34) was chosen to cover the axial crosssection of spinal cord and the surrounding cerebrospinal fluid (CSF). The acquired slice thickness was $1.2 \mathrm{~mm}$ and after reconstruction using k-space zero filling the slice overlap was $0.6 \mathrm{~mm}$. To minimize the number of slices (i.e., left-right field of view), patient position was adjusted to straighten the spine. Measured voxel size was $1.2 \times 1.2 \times 1.4 \mathrm{~mm}$. The keyhole 
fraction was set as high as possible for a dynamic acquisition time of approximately 8 seconds in order to be less than the presumed arterial (AKA) - venous (GARV) circulation time window. This resulted in a keyhole fraction of $45 \%$, which is the fractional surface area of the central part of the acquired k-space in the two phase encoding directions. The resulting acquisition time per dynamic phase varied from 6 to 8.5 seconds. Immediately after acquisition of the reference scan (acquisition time, 11-15 seconds), $45 \mathrm{~mL}$ of contrast medium was injected intravenously at $3 \mathrm{~mL} / \mathrm{sec}$ and flushed with $25 \mathrm{~mL}$ saline, also injected at $3 \mathrm{~mL} / \mathrm{sec}$. Simultaneously the acquisition of 14 dynamic phases was started.

The fundamental difference between elliptic centric and keyhole acquisition in terms of $k$-space coverage is that for the keyhole sampling the central part of $\mathrm{k}$-space
$(45 \%)$ is sampled during passage of the contrast material, while the peripheral part of $k$-space $(55 \%)$ is filled with data that werc acquired before contrast administration. For the elliptic centric acquisition both the central and peripheral part of $\mathrm{k}$-space are sampled during the passage of the contrast material. This means that for the keyhole technique the peripheral k-space data are attenuated in comparison to the corresponding elliptic centric k-space data.

Image analysis. Curved MPR was used to optimally display the spatial course of the AKA, GARV, and/or GPRV. Again, the locations of the AKA, and the GARV and/or GPRV were jointly determined by two observers. For every patient the phases were determined with the earliest and strongest AKA and GARV enhancement.

Table 4.1. Number of patients in whom the signal intensity could be determined and the signal change between first and second phase was positive or negative and mean signal intensities $( \pm 1 \mathrm{SD})$ in the first and second phase images and $\Delta S I$ for various vessels.

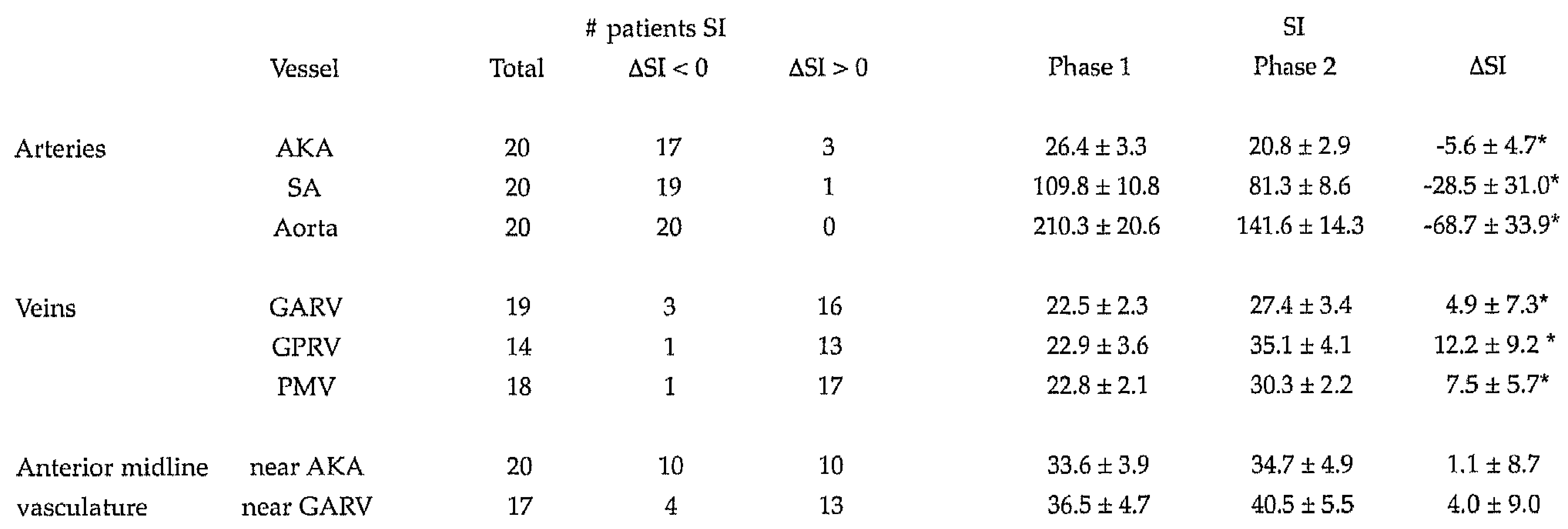

$\mathrm{SI}=$ signal intensity; $\Delta \mathrm{SI}=$ signal changes between first and second phase; $\mathrm{AKA}=$ Adamkiewicz artery; $\mathrm{SA}=$ segmental artery; $\mathrm{GARV}=$ great anterior radiculomedullary vein; GPRV = great posterior radiculomedullary vein; PMV = posterior median vein; ${ }^{*}$ significant signal changes $(P<0.05)$ 


\section{RESULTS}

\section{Two-phase MR angiography}

In all 20 patients the AKA was localized (example in Figure 4.3). The AKA derived from the left sided segmental artery in 11 patients $(55 \%)$. The segmental origin level of the AKA was localized between T8 and L1 in all patients. In 13 patients both the GARV and GPRV were identified, while in 6 patients only the GARV, in 1 patient only the GPRV was detected, and in 1 patient no GARV or GPRV was detected. The GARV was always localized below vertebral level T12 and the GPRV below T10. Table 4.1 lists the signal intensities in the first and second phase images for the various vessels. When averaged over all patients, all arteries showed a significant decrease of SI and the intensities of all veins increased in the second phase. SI changes of the anterior midline vasculature, which was the spatially unresolved combination of arterial and venous enhancement, were not significant. Signal decrease in the AKA occurred in 17 out of 20 patients $(85 \%)$. Temporal differentiation, i.e. signal decrease in the AKA together with an increase in the GARV or GPRV, was achieved in 14 out of 20 patients $(70 \%)$. Thus, differentiation between inlet artery (i.e., the AKA) and outlet vein (either the GARV or GPRV) had to rely in 6 out of 20 patients (30\%) on knowledge of the spinal cord vascular anatomy. The CNR of the AKA was $5.2 \pm 2.5$ (mean $\pm 1 S D$ ) in the first phase and decreased to $3.1 \pm 2.1$ in the second phase. For the GARV the CNR increased from $4.8 \pm 3.5$ to $6.2 \pm 4.1$. The mean bolus arrival time was 25 seconds (range, 18-35 seconds). No significant correlation between signal intensity changes in the AKA or GARV and either acquisition time or bolus arrival time was found $(P>0.2)$.

\section{Computer Model}

Modelling of elliptic centric k-space filling for a bolus contrast distribution showed that the signal intensity decreased for decreasing vessel diameter for both arteries and veins (Figure 4.4). For smaller diameters the decrease in signal intensity was much stronger for arteries than veins, due to the strong bolus shape in the arteries (and not the veins). This resulted in a lower arteriovenous contrast for smaller vessels. Consequently, venous suppression was less effective for vessels with small diameters than for large diameters. Decreasing the acquisition time increased the signal intensity of a small (one pixel thick) artery, decreased the venous signal intensity (Figure 4.5), and consequently increased the arteriovenous contrast. The signal of arteries or veins with relatively large diameters was hardly affected by changing the acquisition time.

\section{Time-resolved $M R$ angiography}

In all subjects the AKA could be identified with time-resolved MR angiography. The AKA originated from the left side in 8 out of 11 subjects $(73 \%)$. For all subjects the level of origin was localized between T8 and L1. In 5 patients both the GARV and GPRV were identified, while in 6 patients only the GPRV was detected. Dynamic separation of the AKA and GARV and/or GPRV was achieved in all subjects. Enhancement of the GARV and/or GPRV occurred at least one phase (6-8.5 seconds) later than the enhancement of the AKA (Figure 4.6 and 4.7 and Table 4.2). In two patients enhancement of the GARV occurred one phase before GPRV enhancement.

The quality of the time-resolved MR angiography images was less compared to the conventional two-phase MR angiography 


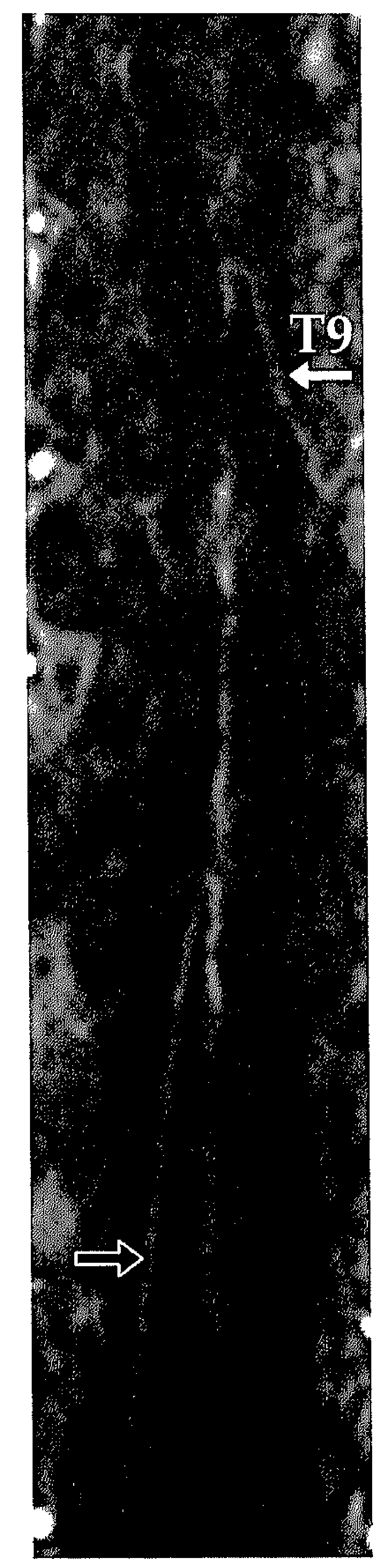

a

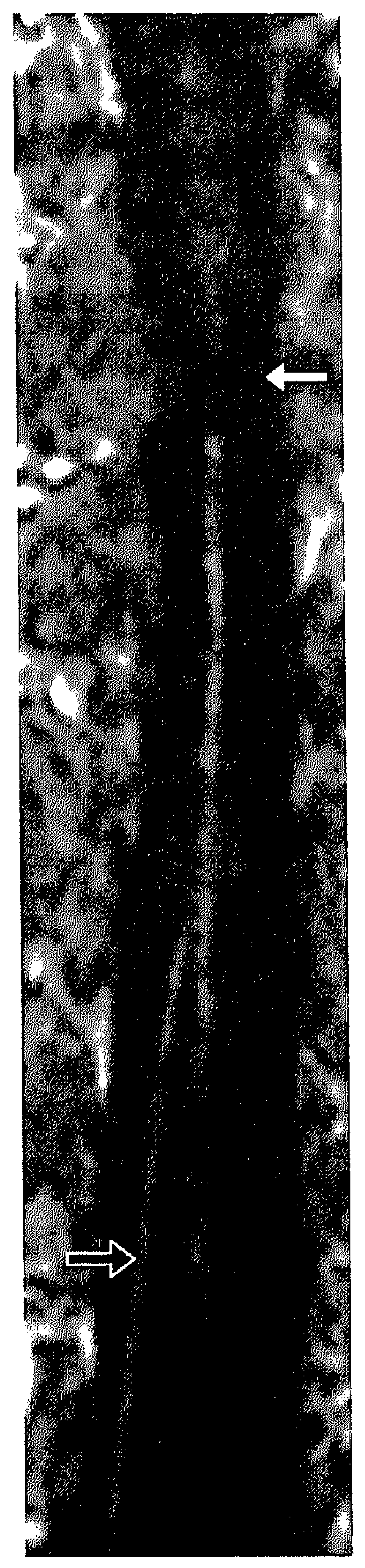

b

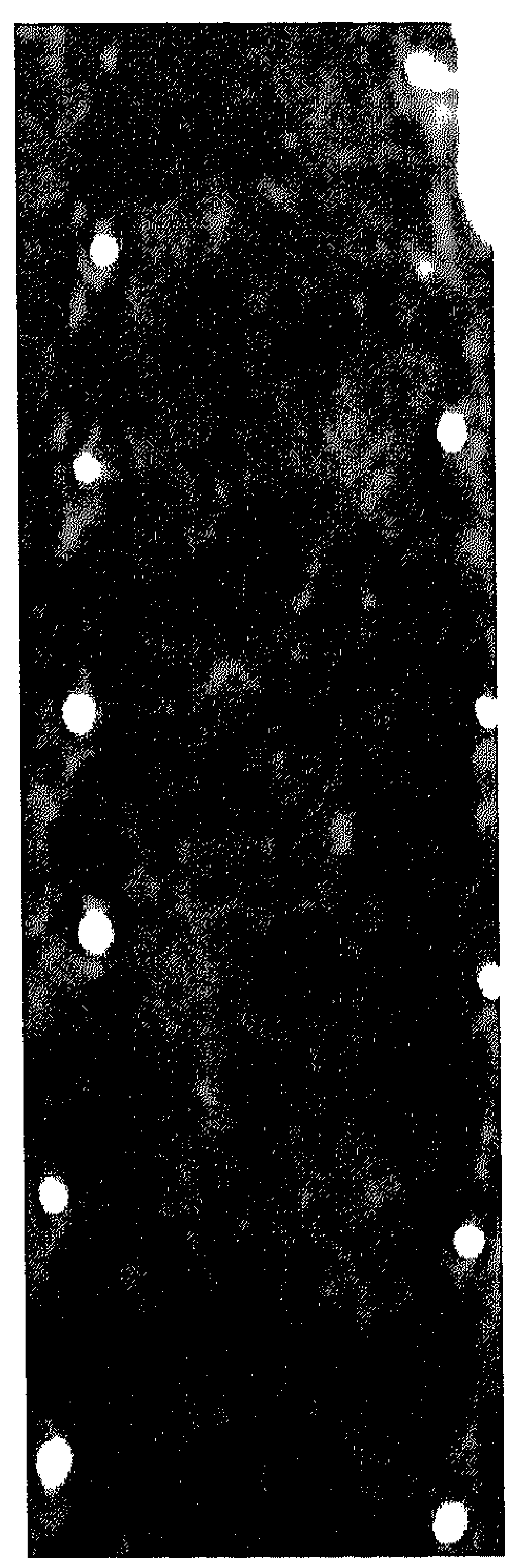

C

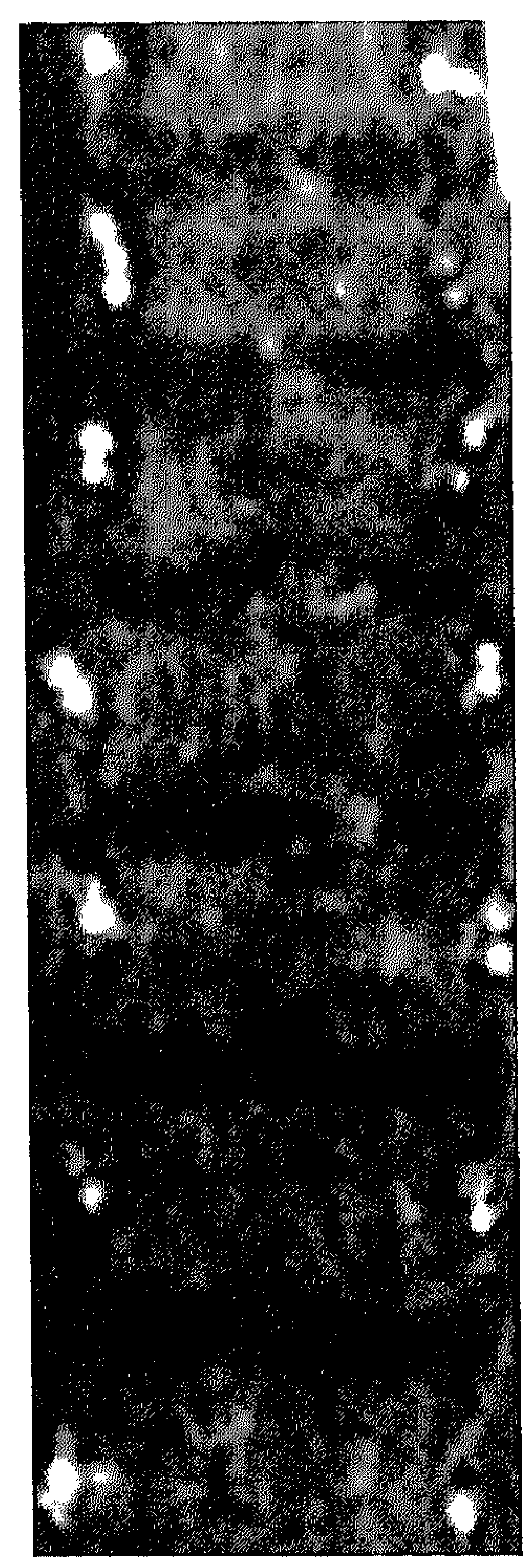

d

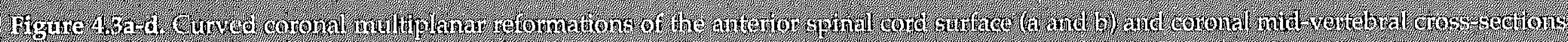

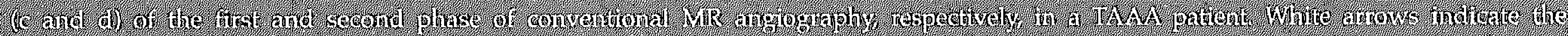

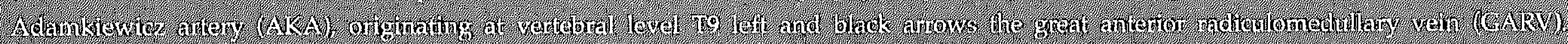

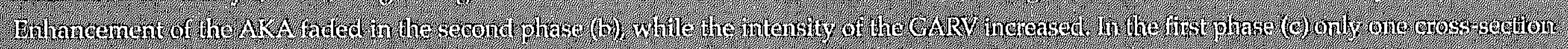

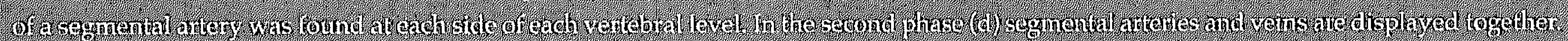


(Figure 4.6). The vessels visualized with timeresolved $M R$ angiography appeared more broadened and less delineated, and signal-tonoise characteristics were less favorable compared to conventional MR angiography due to the shorter acquisition time.

\section{DISCUSSION}

This study was set out to image spinal cord vessels and focused on the differentiation between the spinal cord inlet artery (i.e the AKA) and outlet veins (GARV and/or GPRV). To this end, two MR angiography approaches were investigated that allowed arteriovenous differentiation based on time differences in contrast bolus enhancement. Using a conventional two-phase approach, with 38-55 seconds lasting acquisition times and a large spatial coverage, both spinal cord arteries and veins were visualized on the early phase images. Differentiation between arteries and veins was possible for most patients by comparing their relative signal intensity changes from the early to the late phase images. Complete separation of the AKA and the outlet vein was achieved using the timeresolved approach featuring an acquisition time shorter than the arteriovenous time window of approximately 10 seconds.

For relatively large vessels, such as the aorta and vena cava or the segmental arteries and veins, angiographic arteriovenous separation was proven to be successful with elliptic centric k-space sampling6,16,23. With elliptic centric k-space sampling the center of k-space, where relatively thick vessels were strongly represented, was strongly emphasized compared to the periphery. Using a sharp contrast bolus the periphery of the k-space was filled

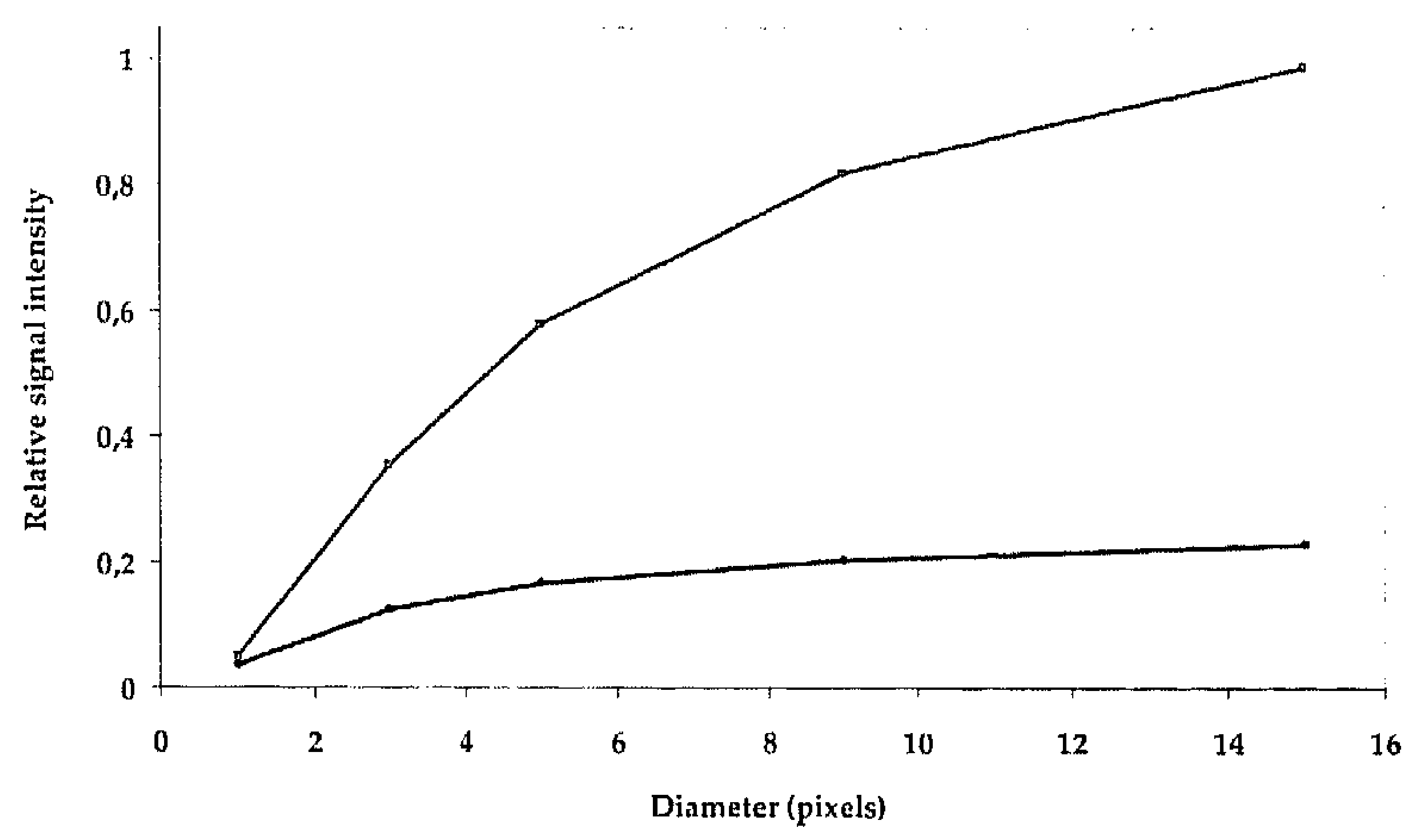

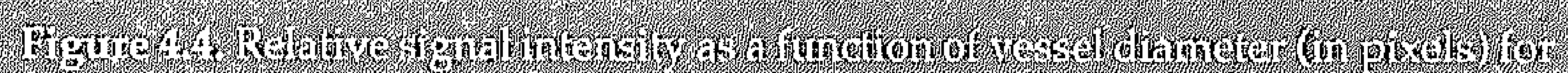

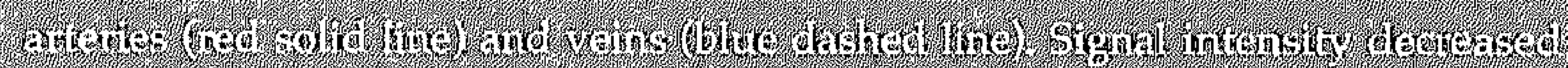

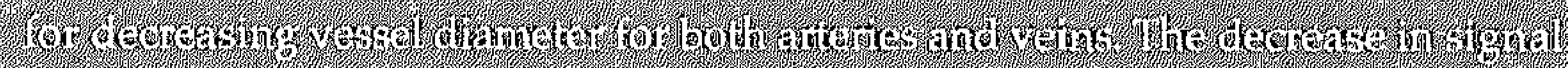

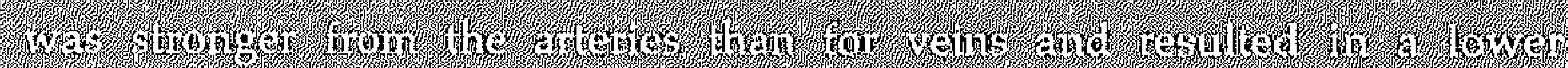

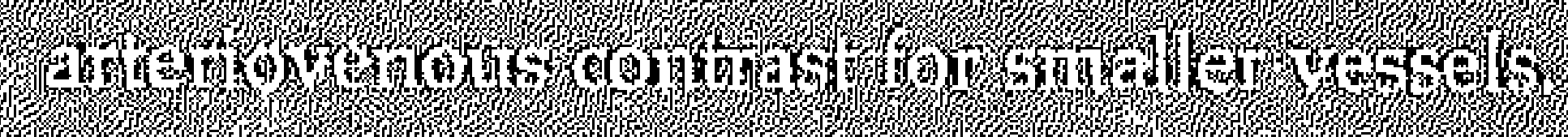

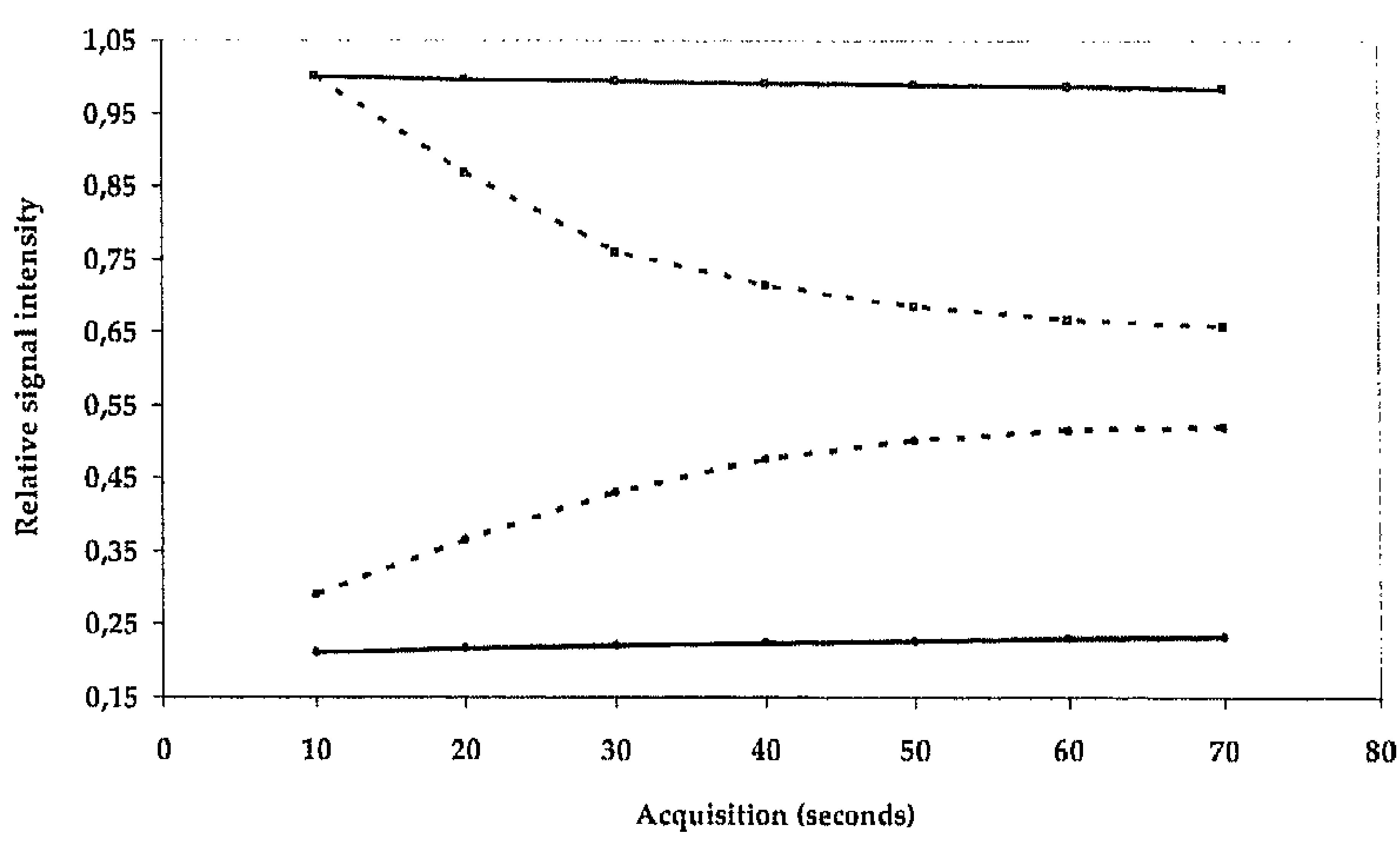

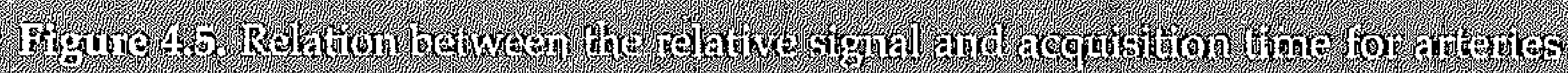

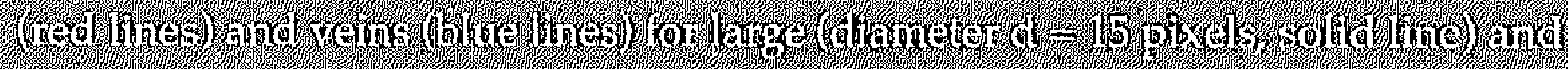

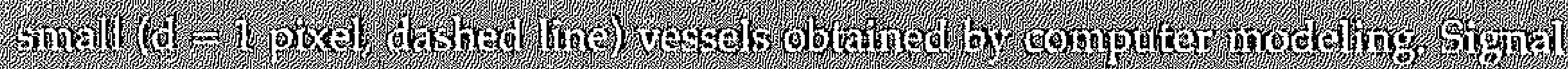

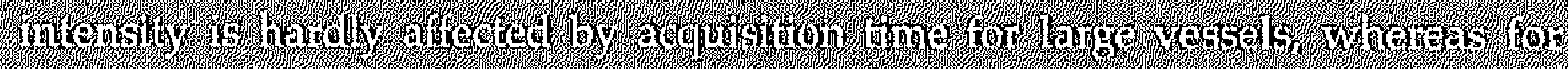

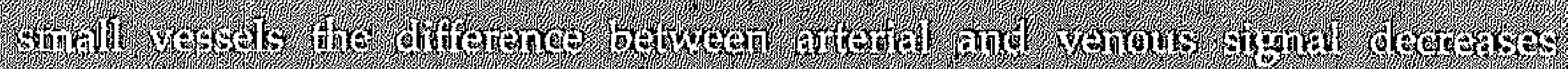

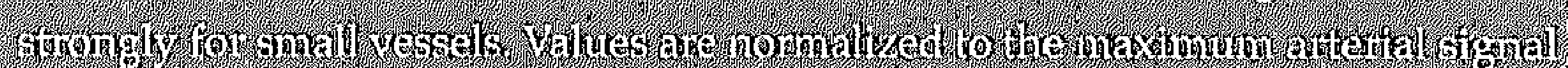

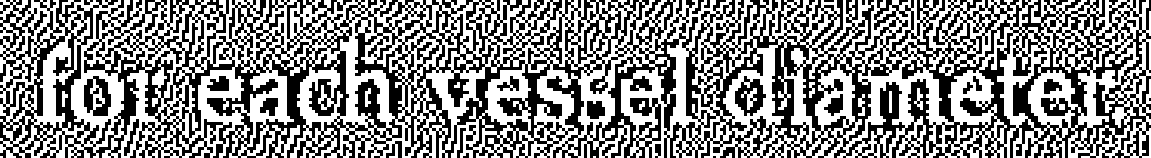


with strongly attenuated contrast data, which had little or no effect on the representation of large vessels For the relatively small vessels of the spinal cord, which are represented in both the center and the periphery of the k-space, the attenuated data in the periphery of $k$-space had therefore a considerable influence on vessel intensity.

A simple computer model was used to demonstrate the essentials of the effect of elliptric centric $\mathrm{k}$-space sampling of a contrast bolus. A shorter acquisition time led to a better arteriovenous contrast. The model also showed that the contrast effect was dependent on the diameter of the considered arteries and veins. As larger arteries and veins were more strongly represented in the $k$-space center, where the bolus enhancement is strongest, the arteriovenous contrast is best for large arteries and veins. For relatively small arteries and veins the $k$-space representation is more or less equally strong in the center and periphery of the $k$-space and the correspondingly obtained contrast was less, as only the center was emphasized by the contrast bolus. It should be remarked that although the model explained the effects of the centric k-space sampling in relation to the dynamics of contrast bolus it was not meant to accurately predict the in vivo contrast values between arteries and veins or actually simulate the complex vascular physiology and anatomy of the spinal cord.

Table 4.2. Overview of the time-resolved MR angiography characteristics and observations.

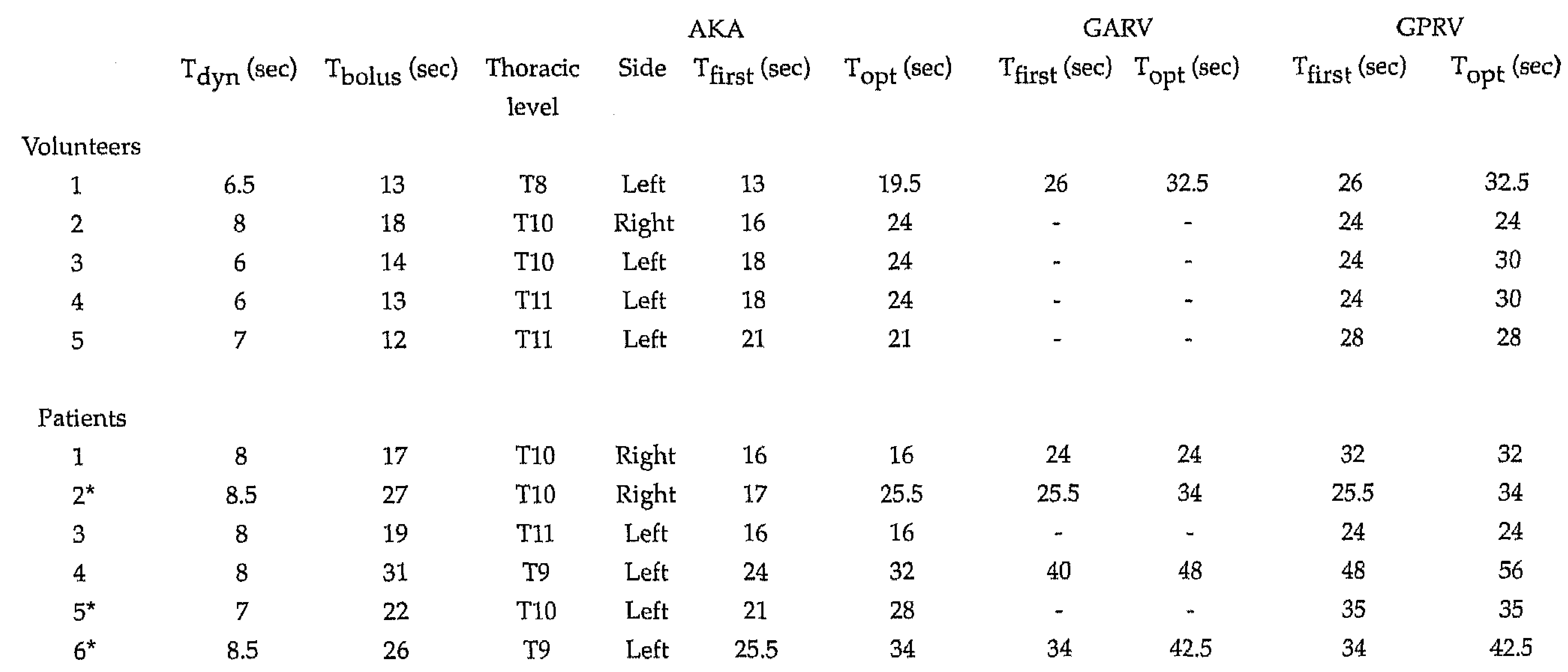

$\mathrm{T}_{\mathrm{dyn}}=$ acuisition time of one dynamic phase; $\mathrm{T}_{\text {bolus }}=$ arrival time of the $2 \mathrm{~mL}$ contrast bolus in the abdominal aorta; $\mathrm{AKA}=\mathrm{Adamkiewicz}$ artery;

$\mathrm{T}_{\text {first }}=$ time of first vessel enhancement; $\mathrm{T}_{\mathrm{opt}}=$ time of optimal vessel enhancement; $\mathrm{GARV}=$ great anterior radiculomedullary vein; $\mathrm{GPRV}=$ great posterior radiculomedullary vein; * patients who also underwent a conventional MR angiography exam 


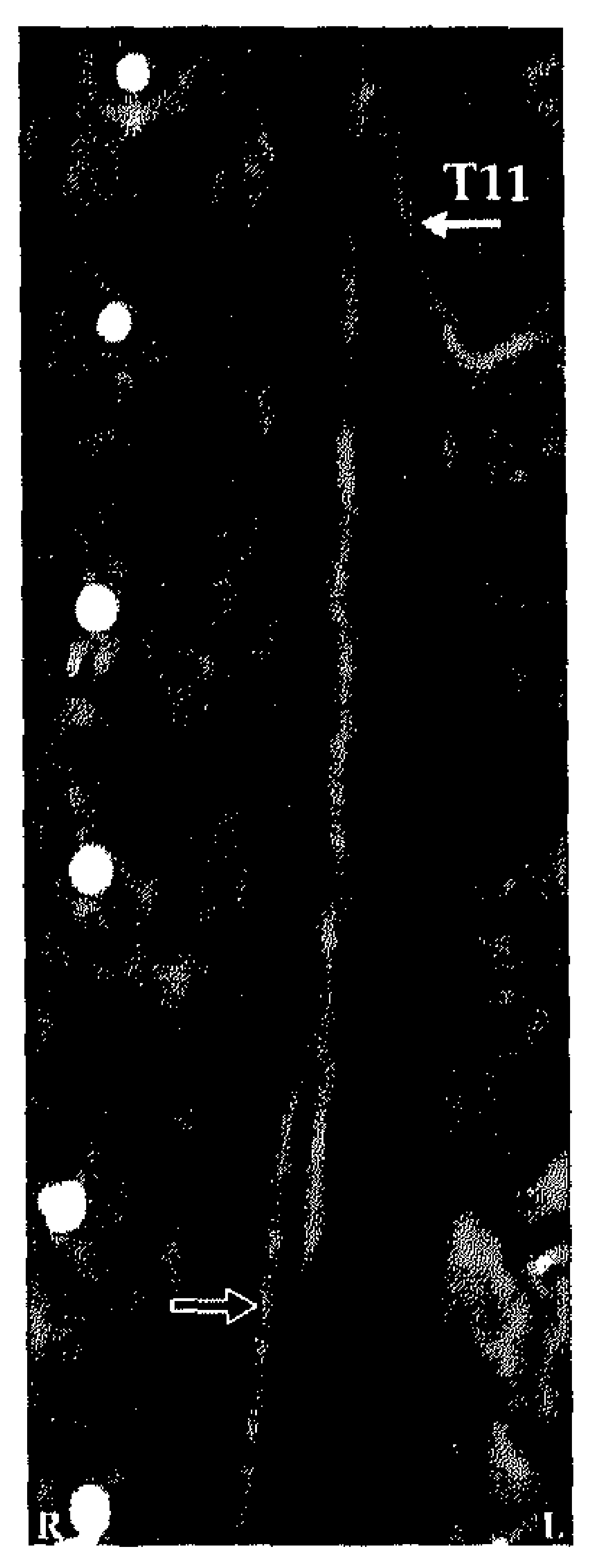

a

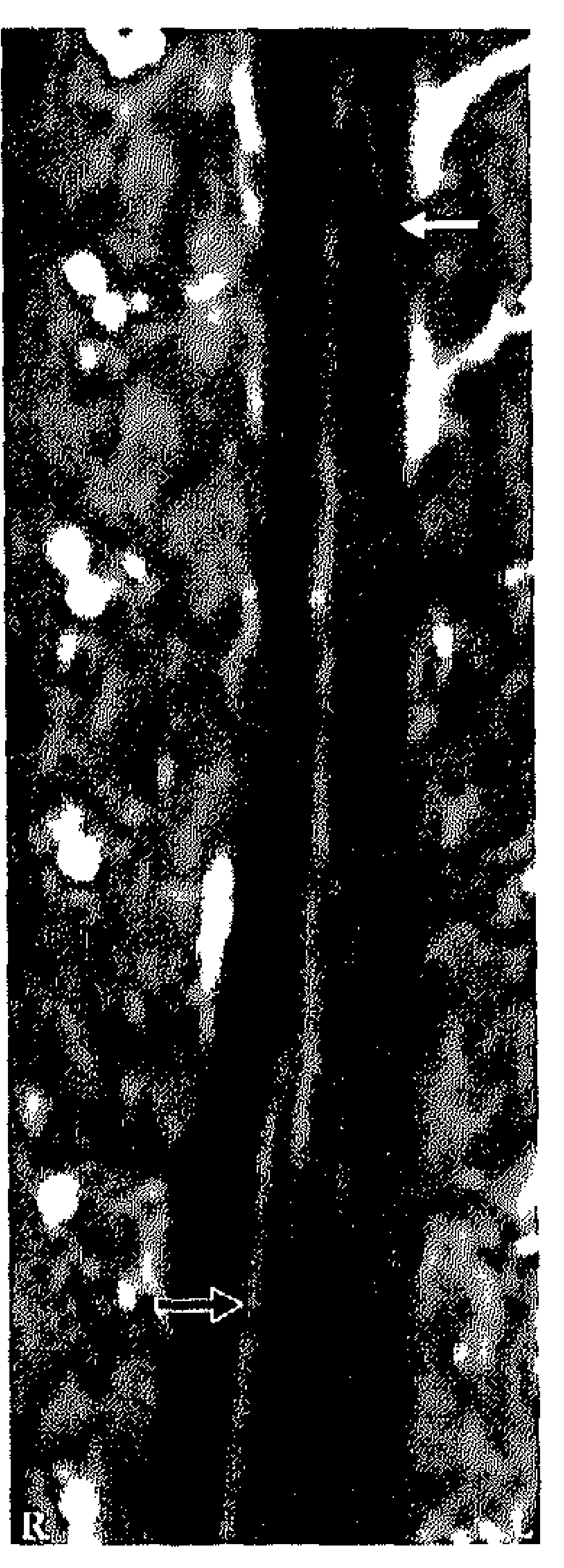

b

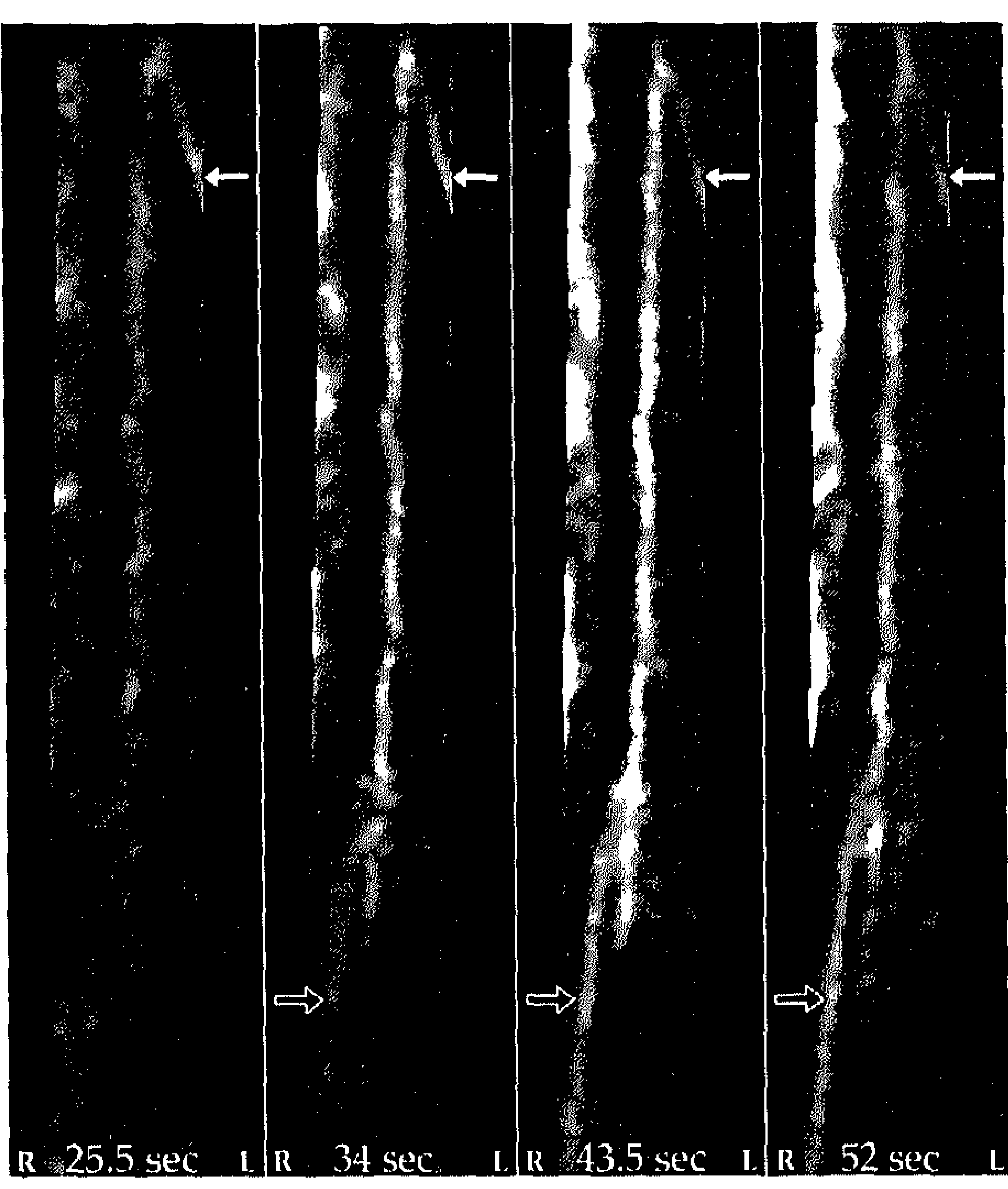

C

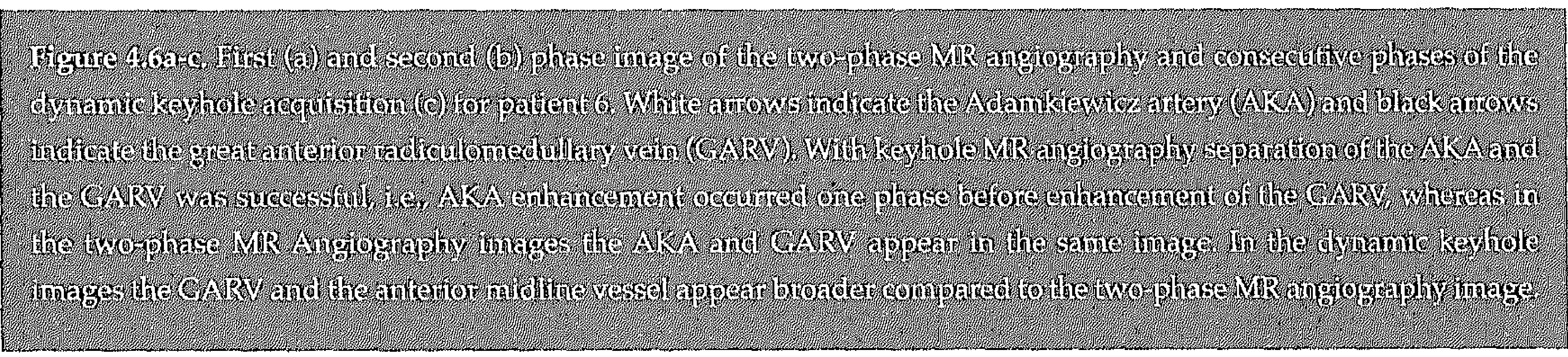

Using our conventional two-phase MR angiography protocol with elliptic centric $\mathrm{k}$ space ordering, spinal cord veins appeared already enhanced in the early phase image. Veins were depicted even though the center of $\mathrm{k}$-space was acquired when the venous contrast agent concentration was still low relative to the arterial concentration. Comparing the first and second phase images provided information on the differences in early and late contrast enhancement. When a vessel appeared bright in the first dynamic phase compared to the second, it was classified as an artery. However, the measured signal changes appeared rather small compared to the noise level and quantitative signal change analysis was not conclusive in all patients. This emphasized the need for even shorter acquisition times to enable separation of the AKA from the GARV. 


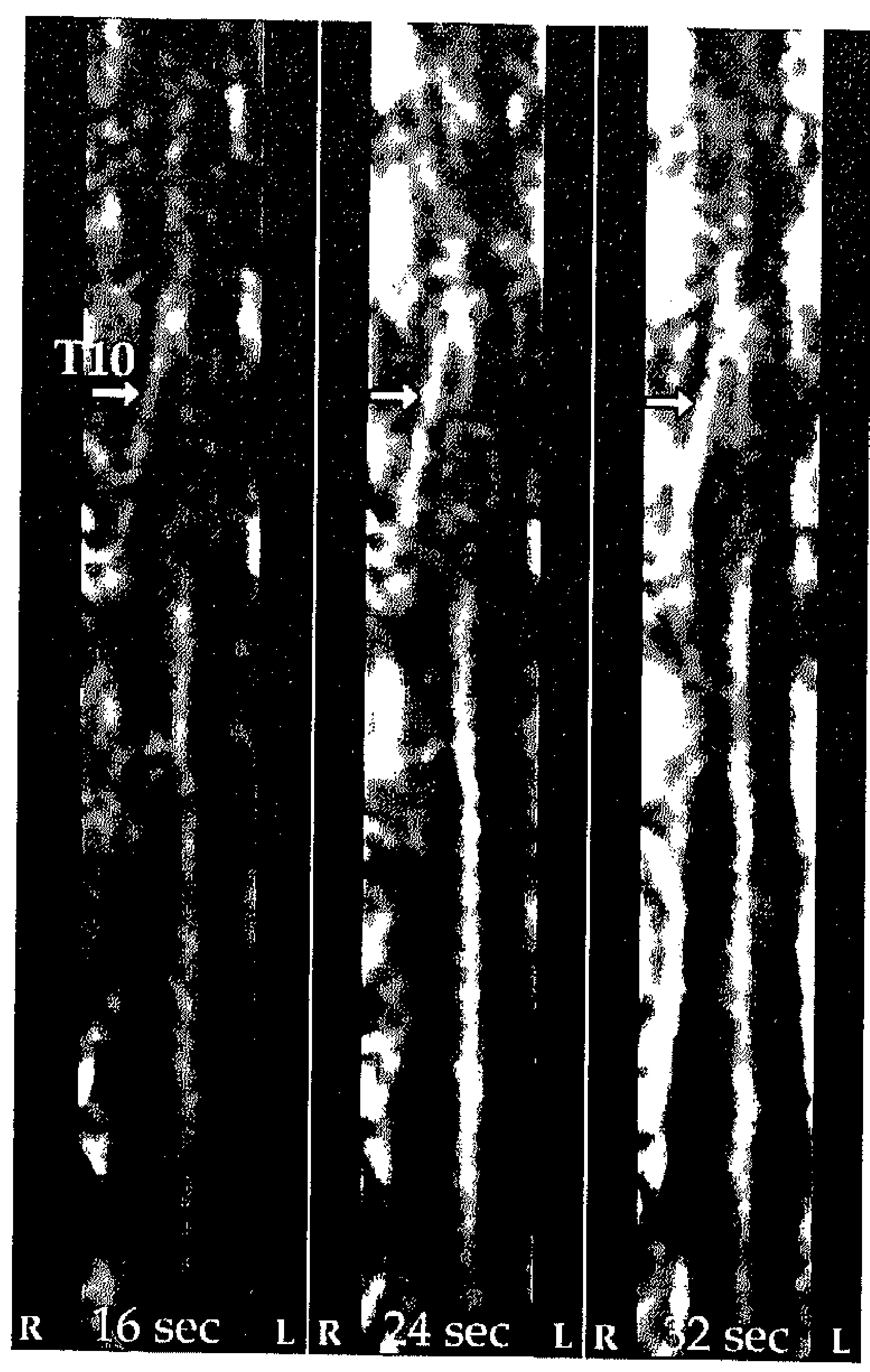

a
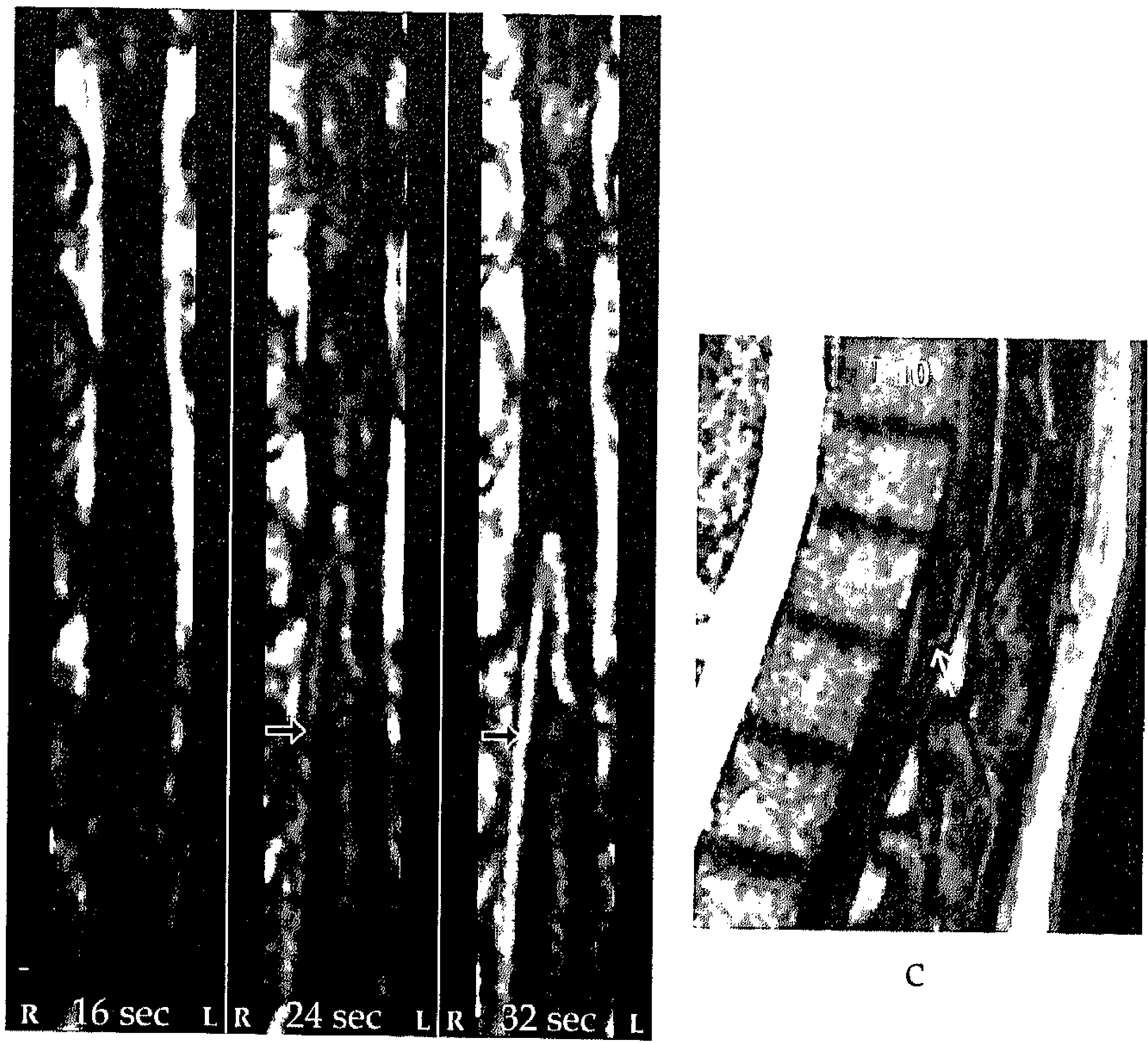

C

$\mathrm{b}$

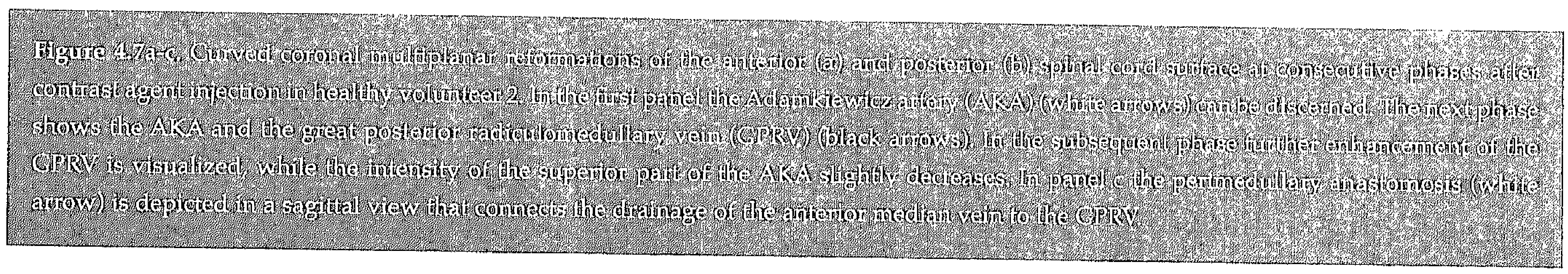

Practically, there were two different ways used to shorten the acquisition time. The first way was to reduce the spatial coverage associated with the phase encoding steps. In acquisition protocol used the largest spatial dimension was required along the craniocaudal axis to accommodate for the interindividual variability of the segmental blood supply to the AKA. Positioning the read-out direction along the largest dimension was most time efficient, but coverage reduction in this direction did not result in shorter acquisition times. To shorten the acquisition time the number of phase encoding steps, positioned in either the anterior-posterior direction (i.e., RFOV) or the left-right direction, was reduced. The fold-over effects of the contrast-enhanced heart and patho- 
logically dilated aorta limited a decrease in RFOV. Therefore, the most effective way to reduce the acquisition time was to limit the number of phase encoding steps in the leftright direction, thus limiting the number of slices.

The second way to reduce the acquisition time was to acquire only a certain fraction of $\mathrm{k}$-space. Because the main interest was dynamic changes in signal intensities (and not vessel delineation) the most intuitive way was to sample the central part of k-space, which determined the main image contrast. For keyhole imaging the acquisition time was decreased by acquiring only the center of k-space in the dynamic phases and complementing the periphery of $\mathrm{k}$-space with pre-contrast data. Previously, keyhole imaging was successfully applied in bolus tracking measurements 24 , contrast medium uptake studies $^{21,25}$, and perfusion ${ }^{26}$ imaging. It was noted from the latter three studies and a simulation study by Spraggins ${ }^{27}$ that the technique works best for dynamic imaging of large objects. The error in object intensity increases for smaller objects and smaller keyhole size. In other words, depiction of small vessels is a general concern with keyhole imaging when using small keyhole fractions. To allow visualization of small (submilimeter) vessels, in the current study the keyhole fraction was chosen as large as possible, resulting in a fraction of $45 \%$.

An improvement on the keyhole technique might be the technique of Time-Resolved Imaging of Contrast Kinetics or TRICKS ${ }^{28}$. With TRICKS, k-space was divided in multiple segments. The central section was updated most frequently, but the more peripheral parts were also regularly refreshed. This dynamic information about the periphery of $\mathrm{k}$-space might improve the effective spatial resolution. However, the spatial resolution achieved with our protocol, which was also affected by a slightly larger voxel size was sufficient to detect and localize the AKA in all patients.

A further option for decreasing acquisition time is parallel imaging, e.g. sensitivity encoding (SENSE) ${ }^{29}$, which uses information about the spatial distribution of the sensitivity of multiple individual coil elements. However, for the thoracolumbar spinal cord, existing coil systems suitable for parallel imaging have large coil elements to create spatial overlap but are therefore less sensitive and provide less craniocaudal coverage than the spine phased-array coil used in this study.

The time interval observed in our measurement between early enhancement of the AKA and the GARV varied between 6 and 14 seconds. This corresponded very well to the estimated time of 10 seconds by Bowen's catheter angiography study ${ }^{18}$. According to Launay et $\mathrm{al}^{30}$ the variation in observed arteriovenous time intervals could likely be attributed to differences in general hemodynamics.

Fried et al ${ }^{1}$ showed that the arteriovenous time window from the ASA to the AMV was 1 to 1.5 seconds. Besides the limited spatial resolution also the rapid filling of the AMV hindered separation of the ASA and AMV. However, the broadening of the anterior and posterior midline vasculature was likely related to the venous filling.

The locations of the segmental artery supplying the AKA found in our study were consistent with results of previous anatomic studies. The left-sided predominance reported by Koshino et al (left-sided in $72 \%$ of the cases $^{31}$ ) was also found in our subjects (left- 
sided in 19 out of 31 subjects, 61\%). All our subjects had an AKA originating between T8 and L1. In Koshino's study, this was the range from which the AKA originated in $91 \%$ of the cases.

Although the focus of this study was on the arteries and veins of the anterior spinal cord, the posterior veins were regarded as well. In our conventional two-phase MR angiography study the GARV and GPRV was detected in 19 and 14 out of 20 patients, respectively. However, when veins were observed on the anterior and posterior surface in one subject, the signal intensities in the GPRV were higher in both the first and second phase images than the GARV. This suggests that the GPRV had a larger diameter than the GARV. However, it should be remarked that the size of the population investigated is limited and that strong variations of the vascular anatomy, particularly for the veins, among subjects exist in the spinal cord. With our time-resolved MR angiography protocol we were able to identify a GPRV in all and a GARV in 5 out of 11 subjects. Similarly, Bowen et al ${ }^{18}$ could always identify a GPRV and only occasionally the GARV in patients with myelopathy. Our results concurred with findings by Doppman et $\mathrm{al}^{32}$ and Launay et $\mathrm{al}^{30}$ who noted that the posterior venous system is dominant.

Even though time-resolved MR angiography enables temporal separation of spinal cord arteries and veins, at the moment it can only serve as an additional protocol to the conventional two-phase approach with a large left-right spatial coverage for preoperative TAAA patients. This could be helpful in patients were arteriovenous separation based on the conventional two-phase MR angiography exam was unclear. The additional time-resolved exam should be performed on another day when the (first) $45 \mathrm{~mL}$ dose contrast material has been excreted. The spatial coverage in the time-resolved $M R$ angiography approach was sufficient to image intradural vessels. However, the trajectory of the segmental arteries connecting the AKA to the aorta was not visualized. For TAAA surgery this (extradural) trajectory represents very important information. As the connection between the aorta and the segmental artery directly supplying the AKA might be occluded by atherosclerosis, one has to verify the patency of the trajectory and detect other segmental arteries possibly supplying the AKA by collaterals 33,34 . Another application of the time-resolved technique might be to introduce the keyhole fraction in our conventional two-phase protocol. Even when the resulting decrease in acquisition time will not be sufficient to achieve complete arteriovenous separation, it likely causes a more distinctive difference in signal change between first and second phase images for arteries and veins.

One could argue that a possible limitation of the current study is the lack of validation by catheter cine angiography. However, catheter angiography is generally not recommended due to its low and variable sensitivity for TAAA patients and the risk for occasional iatrogenic paraplegia9.

For future clinical applications of timeresolved MR angiography, it would be very interesting to study patients with abnormal arteriovenous circulation of the spinal cord. At the moment arteriovenous fistulae can already be reasonably well depicted and localized using the conventional $M R$ angiography technique. Time-resolved MR angiography could potentially provide information on the hemodynamics of the spinal cord circulation, 
which may be deviant in patients with (pial) arteriovenous malformations and arteriovenous fistulae. Particularly for arteriovenous malformations with multiple possible feeders, the temporal resolution of the conventional MR angiography is insufficient to allow separate depiction of all individual feeders. Insight into the drainage pattern can provide important information regarding pathophysiology and treatment. The higher temporal resolution might provide this additional important information. For cerebral vascular malformations time-resolved MR angiography is already used, applying both keyhole imaging and parallel imaging to accelerate the acquisition $35-38$. However, whether timeresolved MR angiography is suitable for this purpose in the spinal cord remains to be proven in future studies.

One might be concerned about the relatively high dose of contrast material. However, the $45 \mathrm{~mL}$ of contrast agent in combination with the fast injection rate, as used in the current study, is reported to be safe 39,40 .

In conclusion, it was shown that differentiation of spinal cord arteries and veins is possible by comparing two subsequent dynamic MR angiography phases. However, for complete arteriovenous separation of small intradural vessels, the acquisition time should be decreased by lowering the number of phase encoding steps. Using time-resolved $M R$ imaging enhancement of the AKA was visualized at least one phase earlier than the GARV. 


\section{REFERENCES}

1. Fried LC, Doppman JL, Di Chiro G. Venous phase in spinal cord angiography. Acta Radiol Dingn (Stockh) 1971;11:393-401.

2. Borst $H G$, Jurmann $M$, Buhner $B$, Laas J. Risk of replacement of descending aorta with a standardized left heart bypass technique. I Thorac Cardiovasc Surg 1994;107:126-32; discussion 132-3.

3. Hyodoh $\mathrm{H}$, Kawaharada N, Akiba $\mathrm{H}$, et al. Usefulness of preoperative detection of artery of Adamkiewicz with dynamic contrast-enhanced MR angiography. Ratiology 2005;236:1004-9.

4. Kawaharada N, Morishita K, Hyodoh H, et al. Magnetic resonance angiographic localization of the artery of Adamkiewicz for spinal cord blood supply. Ann Thorac Surg 2004;78:846-51; discussion 851-2.

5. Svensson LG, Crawford ES, Hess KR, Coselli JS, Safi HJ. Experience with 1509 patients undergoing thoracoabdominal aortic operations. I Vasc Surg 1993;17:357-68; discussion 368-70.

6. Nijenhuis RJ, Leiner T, Cornips EM, et al. Spinal cord feeding arteries at MR angiography for thoracoscopic spinal surgery: feasibility study and implications for surgical approach. Radiology 2004;233:541-7.

7. Guzman R, Dubach-Schwizer S, Heini $P$, et al. Preoperative transarterial embolization of vertebral metastases. Eur Spine J 2005;14:263-8.

8. Krings T, Mull M, Gilsbach JM, Thron A. Spinal vascular malformations. Eur Rndiol 2005;15:267-78.

9. Kieffer E, Fukui S, Chiras J, Koskas F, Bahnini A, Cormier E. Spinal cord arteriography: a safe adjunct before descending thoracic or thoracoabdominal aortic aneurysmectomy. J Vasc Surg 2002;35:262-8.

10. Williams GM, Roseborough GS, Webb TH, Perler BA, Krosnick T. Preoperative selective intercostal angiography in patients undergoing thoracoabdominal aneurysm repair. J Vasc Surg 2004;39:314-21.

11. Yamada N, Takamiya $M$, Kuribayashi S, Okita $Y$, Minatoya K, Tanaka R. MRA of the Adamkiewicz artery: a preoperative study for thoracic aortic aneurysm. J Comput Assist Tomogr 2000;24:362-8.

12. Nijenhuis RJ, Gerretsen $S$, Leiner T, Jacobs MJ, van Engelshoven JM, Backes WH. Comparison of 0.5-M Gd-DTPA with 1.0-M gadobutrol for magnetic resonance angiography of the supplying arteries of the spinal cord in thoracoabdominal aortic aneurysm patients. J Mngn Reson Imaging 2005;22:136-44.

13. Kawaharada N, Morishita K, Fukada J, et al. Thoracoabdominal or descending aortic aneurysm repair after preoperative demonstration of the Adamkiewicz artery by magnetic resonance angiography. Eur J Cardiothorac Surg 2002;21:970-4.

14. Nijenhuis R, Mull M, Thron A, Wilmink J, Backes W. $M R$ angiography of the great anterior radiculomedullary artery (Adamkiewicz artery) validated by digital subtraction angiography. AJNR Am I Neuroradiol 2006;27:1565-1572.

15. Maki JH, Prince MR, Londy FJ, Chenevert TL. The effects of time varying intravascular signal intensity and k-space acquisition order on three-dimensional MR angiography image quality. J Magn Reson Imaging 1996;6:642-51.

16. Wilman $\mathrm{AH}$, Riederer SJ. Performance of an elliptical centric view order for signal enhancement and motion artifact suppression in breath-hold 3D gradient echo imaging. Magn Reson Med 1997;38:793-802.

17. Holsinger $A E$, Riederer SJ. The importance of phaseencoding order in ultra-short TR snapshot MR imaging. Magn Reson Med 1990;16:481-8.

18. Bowen BC, DePrima S, Pattany PM, Marcillo A, Madsen $P$, Quencer RM. MR angiography of normal intradural vessels of the thoracolumbar spine. AJNR Am J Neurorndiol 1996; 17:483-94.

19. $\mathrm{Hu} X$. On the "keyhole" technique. J Magn Reson Imaging 1994;4:231.

20. Jones RA, Haraldseth $O$, Muller TB, Rinck PA, Oksendal AN. K-space substitution: a novel dynamic imaging technique. Mngn Reson Med 1993;29:830-4. 
21. van Vaals JJ, Brummer ME, Dixon WT, et al. "Keyhole" method for accelerating imaging of contrast agent uptake. I Magn Reson Imnging 1993;3:671-5.

22. Willinek WA, Gieseke J, Conrad R, et al. Randomly segmented central k-space ordering in high-spatialresolution contrast-enhanced MR angiography of the supraaortic arteries: initial experience. Radiology 2002;225:583-8.

23. Huston J, 3rd, Fain SB, Riederer SJ, Wilman AH, Bernstein MA, Busse RF. Carotid arteries: maximizing arterial to venous contrast in fluoroscopically triggered contrast-enhanced MR angiography with elliptic centric view ordering. Radiology 1999;211:265-73.

24. Melhem ER, Caruthers SD, Faddoul SG, Tello R, Jara $H$. Use of three-dimensional MR angiography for tracking a contrast bolus in the carotid artery. AJNR Am J Neuroradiol 1999;20:263-6.

25. Kucharczyk W, Bishop JE, Plewes DB, Keller MA, George S. Detection of pituitary microadenomas: comparison of dynamic keyhole fast spin-echo, unenhanced, and conventional contrast-enhanced MR imaging. AJR Am J Roentgenol 1994;163:671-9.

26. Heiland S, Margosian P, Benner T, Reith W, Forsting M, Sartor K. Does the "keyhole" technique improve spatial resolution in MRI perfusion measurements? A study in volunteers. Neurorndiology 2001;43:518-24.

27. Spraggins TA. Simulation of spatial and contrast distortions in keyhole imaging. Magn Reson Med 1994;31:320-2.

28. Korosec FR, Frayne R, Grist TM, Mistretta CA. Timeresolved contrast-enhanced $3 \mathrm{D}$ MR angiography. Magn Reson Med 1996;36:345-51.

29. Pruessmann KP, Weiger $M$, Scheidegger MB, Boesiger P. SENSE: sensitivity encoding for fast MRI. Mngn Reson Med 1999;42:952-62.

30. Launay M, Chiras J, Bories J. Angiography of the spinal cord: venous phase. Normal features. Pathological application. J Neuroradiol 1979;6:287-315.
31. Koshino T, Murakami G, Morishita K, Mawatari T, Abe T. Does the Adamkiewicz artery originate from the larger segmental arteries? I Thorac Cartiounsc Surg 1999;117:898-905.

32. Doppman JL, Di Chiro G, Ommaya AK. Selective arteriography of the spinal cort. St. Louis: W. H. Green, 1969:3-17.

33. Jacobs MJ, de Mol BA, Elenbaas T, et al. Spinal cord blood supply in patients with thoracoabdominal aortic aneurysms. I Vasc Surg 2002;35:30-7.

34. Yoshioka K, Ninuma H, Kawazoe K, Ehara S. Threedimensional demonstration of the collateral circulation to the artery of Adamkiewicz via internal thoracic artery with 16-row multi-slice CT. Eur I Cartiothorac Surg 2005;28:492.

35. Gauvrit JY, Oppenheim C, Nataf F, ct al. Threedimensional dynamic MR angiography for the evaluation of radiosurgically treated cerebral arteriovenous malformations. Eur Radiol 2006;16:583-91.

36. Krings T, Hans F. New developments in MRA: timeresolved MRA. Neurorndiology 2004;46 Suppl 2:s214-22.

37. Tsuchiya K, Aoki C, Fujikawa A, Hachiya J. Threedimensional $M R$ digital subtraction angiography using parallel imaging and keyhole data sampling in cerebrovascular diseases: initial experience. Eur Radiol 2004;14:1494-7.

38. Tsuchiya K, Honya $\mathrm{K}$, Fujikawa A, Tateishi $\mathrm{H}_{\text {, }}$ Shiokawa $Y$. Postoperative assessment of extracranialintracranial bypass by time-resolved 3D contrastenhanced $M R$ angiography using parallel imaging AINR Am J Neuroradiol 2005;26:2243-7.

39. Boos M, Scheffler K, Haselhorst R, Reese E, Frohlich J, Bongartz GM. Arterial first pass gadolinium-CM dynamics as a function of several intravenous saline flush and $\mathrm{Gd}$ volumes. I Magn Reson lmaging 2001;13:568-76.

40. Cochran ST, Bomyea K, Sayre JW. Trends in adverse events after IV administration of contrast media. AJR Am J Roentgenol 2001;176:1385-8. 


\section{Chl}

MR angiography of the great anterior radiculomedullary artery (Adamkiewicz artery) validated by digital subtraction angiography

RJ Nijenhuis M Mull

JT Wilmink AK Thron WH Backes

Amorican loumal of Neuromadology $2006,27: 1565-72$ 


\section{ABSTRACT}

Purpose: Imaging of the anterior superficial spinal cord arteries by MR angiography is hindered by their small calibers and the similarity in configuration with the anterior superficial spinal cord veins. To validate the location and spatial configuration of the Adamkiewicz artery (AKA), magnetic resonance (MR) angiography was compared with intra-arterial catheter X-ray angiography.

Materials and Methods: Fifteen patients with suspected spinal cord vascular pathology underwent both spinal MR angiography and selective spinal cord catheter angiography. Two-phase MR angiography was performed with the use of a centric k-space filling scheme synchronized to the contrast bolus arrival. The level and side of the AKA origin were scored on the catheter and MR angiography images and compared regarding image quality in terms of vessel conspicuity, contrast, continuity, sharpness, and background homogeneity on a relative 5-point scale.

Results: Localization and spatial configuration of the AKA by MR angiography was in agreement with catheter angiography findings in 14 of 15 cases. One mismatch of 1 vertebral level (not side) appeared as a result of the tangled vascular pathology. Comparison of image quality revealed that catheter angiography is superior to $M R$ angiography concerning vessel continuity, sharpness, and background homogeneity $(P<0.001)$. Overall vessel conspicuity and contrast were judged to be similar.

Conclusions: MR angiography can visualize and localize the level of the AKA correctly. Image quality of MR angiography is sufficient for detection of the AKA but is inferior to catheter angiography. 


\section{INTRODUCTION}

Because of its high spatial resolution, intra-arterial catheter (X-ray) angiography, also known as digital subtraction angiography, is considered the standard of reference for imaging the very small anterior superficial spinal cord arteries. Catheter angiography is an invasive technique, however, in that it involves selective catheterization and contrast injection into small arteries supplying the spinal cord. This prompts the development of less invasive techniques such as computed tomography $(\mathrm{CT})^{1-3}$ and magnetic resonance (MR) angiography $3-18$. Before introducing these new techniques in a clinical setting it is of absolute importance to validate them.

Imaging of the anterior superficial spinal cord arteries requires simultaneous high spatial and high temporal resolution. The artery that is mainly responsible for the blood supply to the anterior spinal artery (ASA) and thus for the function of the thoracolumbar spinal cord is the great anterior radiculomedullary artery (i.e., the Adamkiewicz artery [AKA] ${ }^{19-20}$. Although this is a minute artery (caliber 0.5-1.0 mm) ${ }^{21}$, it is the largest anterior spinal cord artery. The intervertebral foramen at which the spinal branch of the posterior segmental artery enters the spinal canal and continues as the AKA displays considerable anatomic variance ${ }^{22}$. In addition, when a spinal arteriovenous malformation (SAVM) is to be diagnosed, the entire vertebral column has to be depicted, because an SAVM can arise along this entire region ${ }^{22}$. This variability in vascular anatomy and pathology necessitates the use of a large field of view and thereby precludes achievement of high spatial resolution. The short transit time from arteries to veins of the spinal cord circulation $(<10 \text { seconds) })^{15}$, as well as the spatial similarity in configuration of the anterior spinal cord arteries and veins (Figure 5.1), places further high demands on the temporal resolution of the imaging technique to obtain separation between arteries and veins.

First-pass MR angiography has been shown to visualize the AKA ${ }^{4-8}$. However, catheter angiography verification has only been performed in a few cases ${ }^{6-7}$. Therefore, a systematic validation is of interest. The aim of the current study was to investigate whether two-phase MR angiography is able to detect and localize the AKA with catheter angiography as the standard of reference in patients with suspected spinal cord vascular pathology.

\section{MATERIALS and METHODS}

\section{Patients}

From December 2003 through October 2004, 15 consecutive patients (12 men, 3 women) with suspected spinal vascular abnormalities were included in this study. The mean age was 60 years (range, $43-76$ years). All patients underwent both MR and catheter angiography investigation as part of the normal clinical work-up. Classification of vascular pathology, as listed in Table 5.1, was determined on the basis of catheter angiography. Informed consent was obtained from all patients. MR angiography was performed 1 day before catheter angiography in all patients.

\section{Imaging modalities}

MR angiography. Imaging was performed on a clinical 1.5-Tesla MR imaging system 


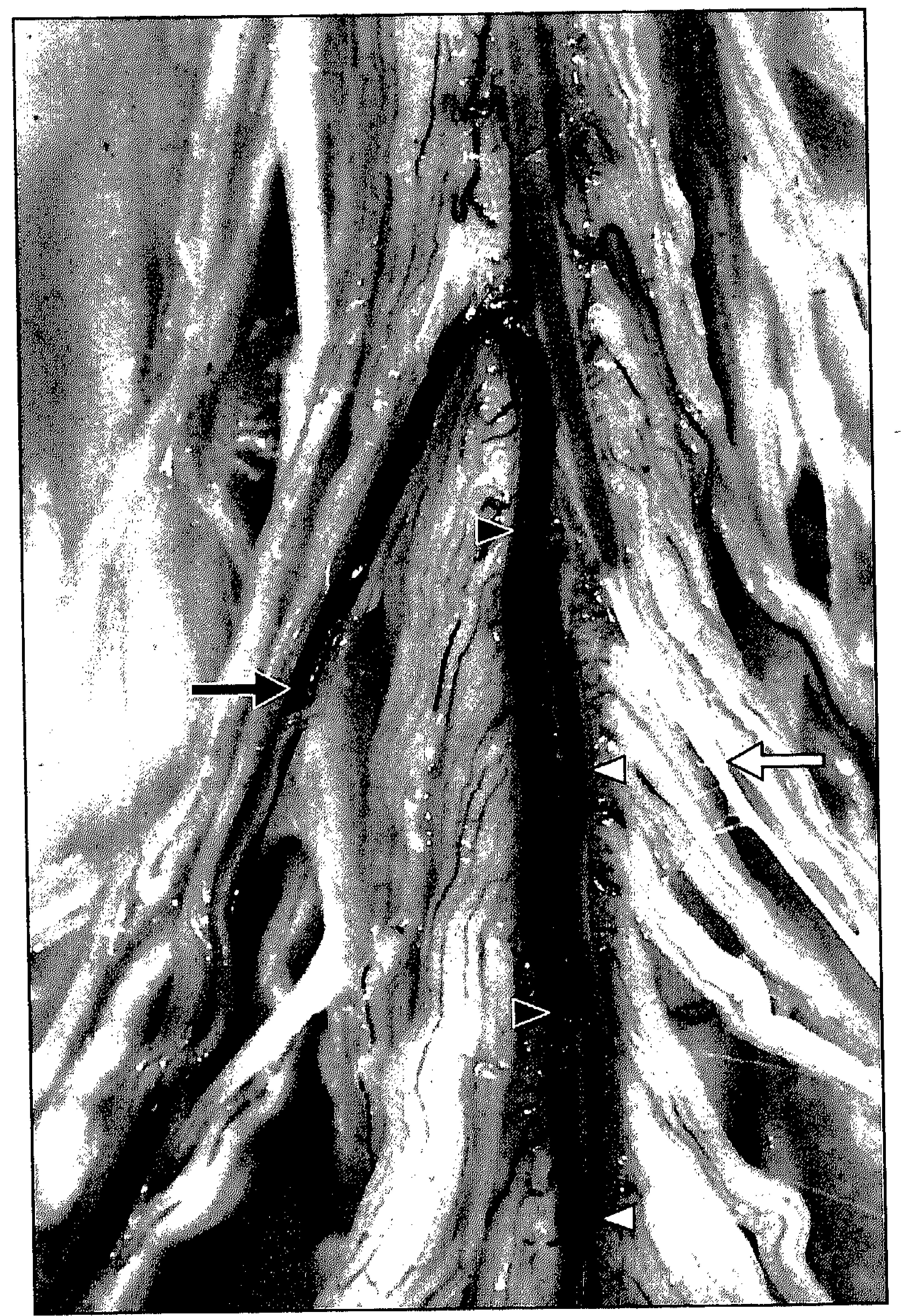

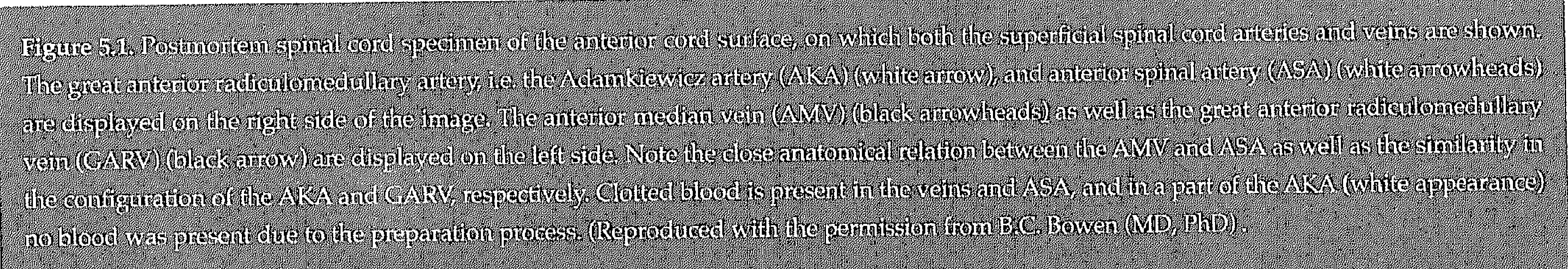


Validation of spinal cord MR angiography

Table 5.1. Patient characteristics and observations for MR and catheter angiography.

\begin{tabular}{|c|c|c|c|c|c|c|c|c|c|c|c|c|}
\hline \multirow[t]{4}{*}{ Case } & \multirow[t]{4}{*}{ Sex } & \multirow[t]{4}{*}{ Age } & \multicolumn{5}{|c|}{ MR angiography } & \multicolumn{4}{|c|}{ Catheter angiography } & \multirow[t]{3}{*}{ Pathology } \\
\hline & & & \multicolumn{2}{|c|}{ AKA } & Other & \multirow{2}{*}{\multicolumn{2}{|c|}{ GARV }} & \multirow{2}{*}{\multicolumn{2}{|c|}{ AKA }} & \multirow{3}{*}{$\begin{array}{l}\text { Other } \\
\text { ARA }\end{array}$} & \multirow[t]{3}{*}{ GARV } & \\
\hline & & & & & ARA & & & & & & & \\
\hline & & & Level & Side & & Level & Side & Level & Side & & & \\
\hline 1 & $\mathrm{M}$ & 44 & T9 & Right & No & $\mathrm{L} 2$ & Right & T9 & Right & $\mathrm{T} 4$ & No & Hemangioblastoma \\
\hline 2 & $\mathrm{M}$ & 62 & $\mathrm{~T} 8$ & Left & No & L3 & Right & $\mathrm{T} 8$ & Left & No & No & SDAVF \\
\hline 3 & $\mathrm{M}$ & 57 & L1 & Right & No & No & No & L1 & Right & No & No & SAVM, fistulous type \\
\hline 4 & $\mathrm{M}$ & 63 & T9 & Left & No & L1 & Right & T9 & Left & No & No & Spinal SAH \\
\hline 5 & $\mathrm{M}$ & 73 & $\mathrm{~T} 12$ & Left & No & L3 & Right & $\mathrm{T} 12$ & Left & T 3 & No & None \\
\hline 6 & $\mathrm{M}$ & 64 & $\mathrm{~T} 12$ & Left & No & $\mathrm{L} 2$ & Left & $\mathrm{T} 12$ & Left & No & No & SDAVF \\
\hline 7 & $\mathrm{M}$ & 48 & T11 & Right & No & L3 & Right & $\mathrm{T} 11$ & Right & No & No & SDAVF \\
\hline 8 & $\mathrm{M}$ & 55 & L1 & Right & No & L3 & Right & L1 & Right & No & No & SDAVF \\
\hline 9 & $\mathrm{M}$ & 72 & T11 & Left & No & $\mathrm{L} 2$ & Right & $\mathrm{T} 11$ & Left & No & No & SDAVF \\
\hline 10 & $\mathrm{M}$ & 66 & $\mathrm{~L} 2$ & Left & No & L2 & Right & $\mathrm{L} 2$ & Left & No & No & SDAVF \\
\hline 11 & $\mathrm{~F}$ & 43 & $\mathrm{~T} 12$ & Left & No & No & No & L1 & Left & No & No & SAVM, fistulous type \\
\hline 12 & F & 43 & $\mathrm{~T} 11$ & Right & No & L3 & Left & T11 & Right & No & No & None \\
\hline 13 & M & 73 & $\mathrm{~T} 10$ & Left & No & L2 & Right & $\mathrm{T} 10$ & Left & No & No & SDAVF \\
\hline 14 & $\mathrm{~F}$ & 76 & $\mathrm{~T} 12$ & Right & No & L3 & Right & $\mathrm{T} 12$ & Right & No & No & SDAVF \\
\hline 15 & $\mathrm{M}$ & 59 & L1 & Left & No & $\mathrm{L} 2$ & Right & L1 & Left & No & No & None \\
\hline
\end{tabular}

$\mathrm{AKA}=$ Adamkiewicz artery $\mathrm{ARA}=$ anterior radiculomedullary artery; $\mathrm{GARV}=$ great anterior radiculomedullary vein; $\mathrm{M}=\mathrm{male} ; \mathrm{F}=$ female; $\mathrm{T}=$ thoracic vertebra; $\mathrm{L}=$ lumbar vertebra; $\mathrm{SDAVF}=$ spinal dural arteriovenous fistula; $\mathrm{SAVM}=$ spinal arteriovenous malformation; $\mathrm{SAH}=$ subarachnoidal hemorrhage

equipped with a phased-array synergy surface spine coil. Patients were imaged in the supine position. The MR imaging protocol comprised 3 different pulse sequences. First, a T2weighted survey scan was acquired to image the spinal cord and vertebral column for anatomic reference. The field of view (FOV) extended from the third thoracic vertebra (T3) to the fifth sacral vertebra (S5). Acquisition parameters were TR $2686 \mathrm{msec}$; TE $120 \mathrm{msec}$; flip angle $90^{\circ}$; craniocaudal FOV $500 \mathrm{~mm}$; matrix size $320 \times 480$ (phase encoding $\times$ readout); 12 sagittal sections; and voxel sizes $1.25 \mathrm{x}$

\section{$1.67 \times 4.0 \mathrm{~mm}$.}

Second, MR fluoroscopy was used to determine the scan delay between the start of contrast bolus injection and the start of the MR angiography pulse sequence. For this purpose, a test bolus of $2.0 \mathrm{~mL}$ of gadopentetate dimeglumine ( $0.5 \mathrm{~mol} / \mathrm{L}$ Gd-DTPA) was administered via the left antecubital vein. The test bolus was administered at the same injection rate of $3 \mathrm{~mL} / \mathrm{sec}$ as the final bolus using a power-injector followed by a saline flush of $25 \mathrm{~mL}$. The MR fluoroscopy sequence consisted of an $80-\mathrm{mm}$ section that was 
sagittally positioned through the aorta. This section was acquired once each second over a period of 2 minutes. The pulse sequence parameters were TR $5.2 \mathrm{msec}$; TE $1.4 \mathrm{msec}$; flip angle $35^{\circ}$; FOV $450 \mathrm{~mm}$; and matrix size $205 x$ 256. Viewing the series of bolus timing images, the scan delay was defined as the time between start of test bolus injection and maximal enhancement of the entire aorta down to the bifurcation. The obtained scan delay was used to synchronize the sampling of the center of k-space with the peak contrast agent enhancement in the subsequent MR angiography acquisition.

Third, for the final MR angiography, a total volume of $45 \mathrm{~mL}(0.3 \mathrm{mmol} / \mathrm{kg}$ of body weight) of contrast agent was administered and flushed with $25 \mathrm{~mL}$ of saline both injected at a rate of $3 \mathrm{~mL} / \mathrm{sec}$. $M R$ angiography consisted of 2 consecutive dynamic phases to obtain an early (ie, first, preferably arterial) phase and a late (ie, second, combined arterial venous) phase 3D image set. Each phase took 36 to 41 seconds, depending on the number of sagittal sections required to cover the entire transverse cross-section of the vertebrae along the entire vertebral column. Sagittal section orientation was preferred over coronal orientation to obtain the least number of phase encoding steps and consequently the shortest acquisition time. The FOV in craniocaudal (read-out) direction was set to $500 \mathrm{~mm}$ to enable the maximum possible anatomic coverage to include (i) the (possibly aberrant) origin of the AKA and (ii) the origin of any possible vascular abnormality. The FOV was $175 \mathrm{~mm}$ in anterior-posterior (phase encoding) direction (rectangular FOV of 35\%). The use of a smaller FOV in the phase encoding direction reduced the number of phase encoding steps and therefore acquisition time. Matrix dimen- sions were $464 \times 512$ (phase encoding $\times$ readout), and pixel size was $0.8 \times 0.8 \mathrm{~mm}$. Acquired sagittal sections were $1.2 \mathrm{~mm}$ and interpolated (zero-filling in k-space) during reconstruction to $0.6 \mathrm{~mm}$ sections. The number of sections was individually adjusted (range, $75-85 ; 45-51 \mathrm{~mm}$ ) to include the entire vertebral column from T3 down to S5 in the craniocaudal direction. Acquisition parameters for the spoiled gradient-echo pulse sequence were TR $5.9 \mathrm{mmsec}$; TE $1.9 \mathrm{msec}$ (partial echo $62.5 \%$ ); and FA $30^{\circ}$. To obtain images in which venous enhancement was suppressed, k-space was filled using elliptical centric ordering in which the first 4 seconds were randomly filled. Imaging was performed without breath-hold.

Catheter angiography. Selective spinal cord catheter angiography was performed by a transfemoral approach. In the frontal view, 2 frames per second were acquired. The manually injected contrast agent (2-3 mL per injection) had an iodine concentration of 300 $\mathrm{mg}$ of iodine/ $\mathrm{mL}$. Radiographic parameters were $80 \mathrm{kV}$; $400 \mathrm{~mA}$; matrix size $1024 \times 1024$; entrance format $11 \times 13 \mathrm{~cm}$; and spatial resolution 2.2 line pairs $/ \mathrm{mm}$. Oblique views were added to elucidate the composition of the fistula zone in case of a spinal dural arteriovenous fistula (SDAVF). For image comparison with first phase MR angiography images, only early (arterially) enhanced images were used. The angiographic protocol was adapted to the underlying vascular pathology. In all cases, spinal cord catheter angiography included identification of the arteries supplying the thoracolumbar region. Classification of pathologic findings was based on catheter angiography findings. 
Validation of spinal cord MR angiography

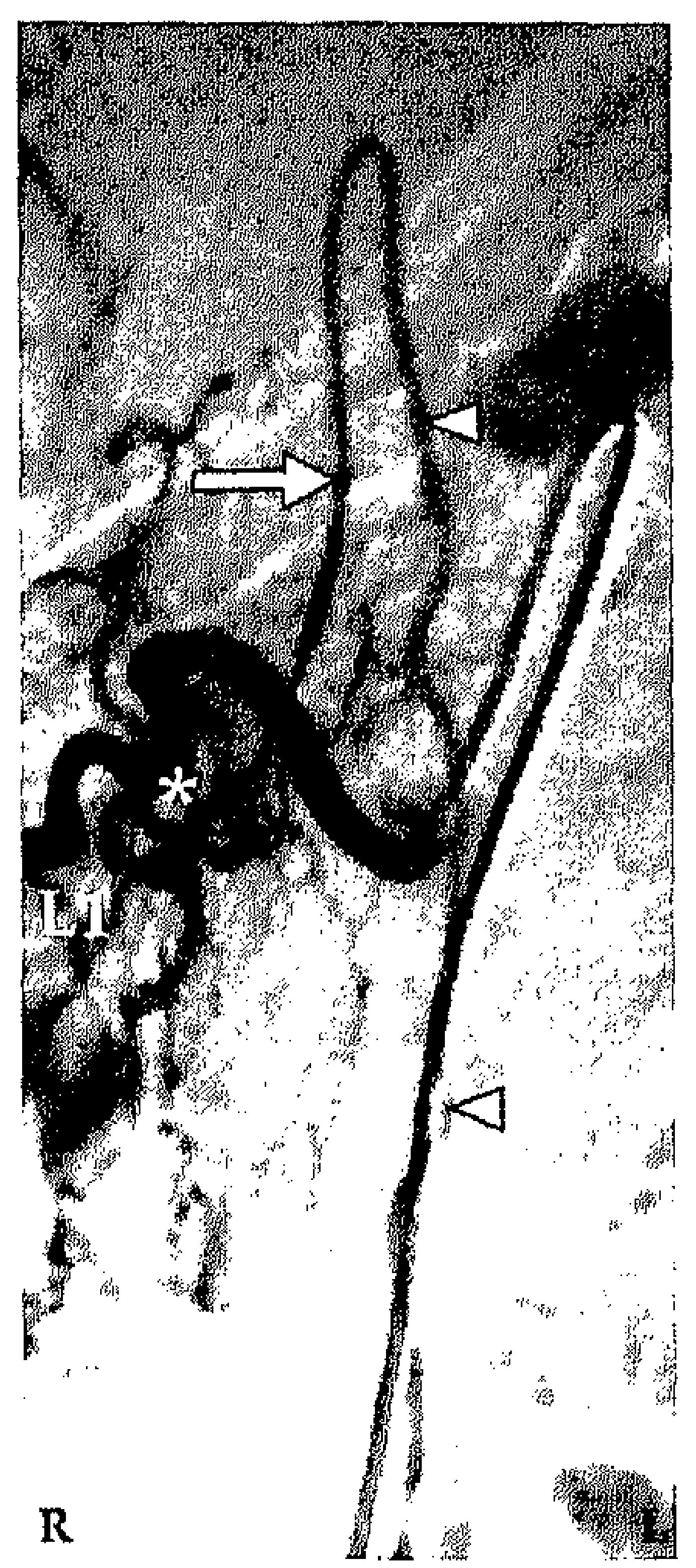

a

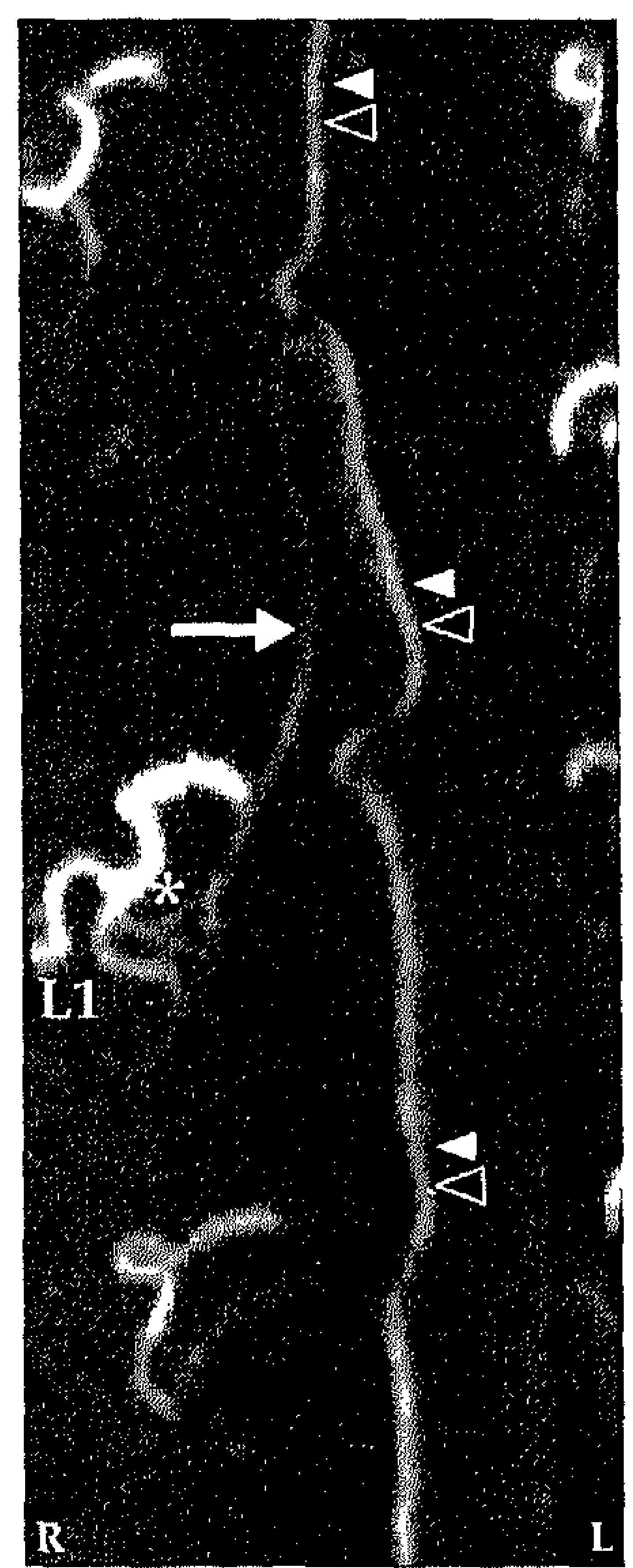

b

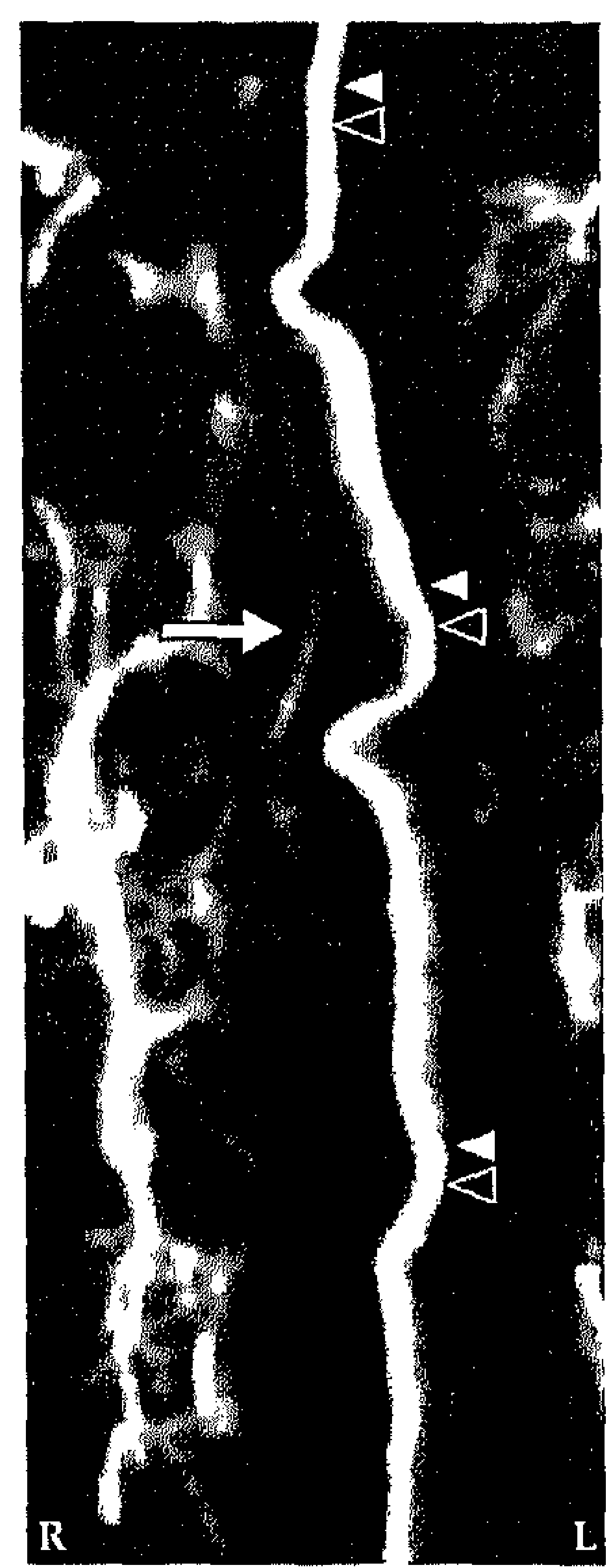

C

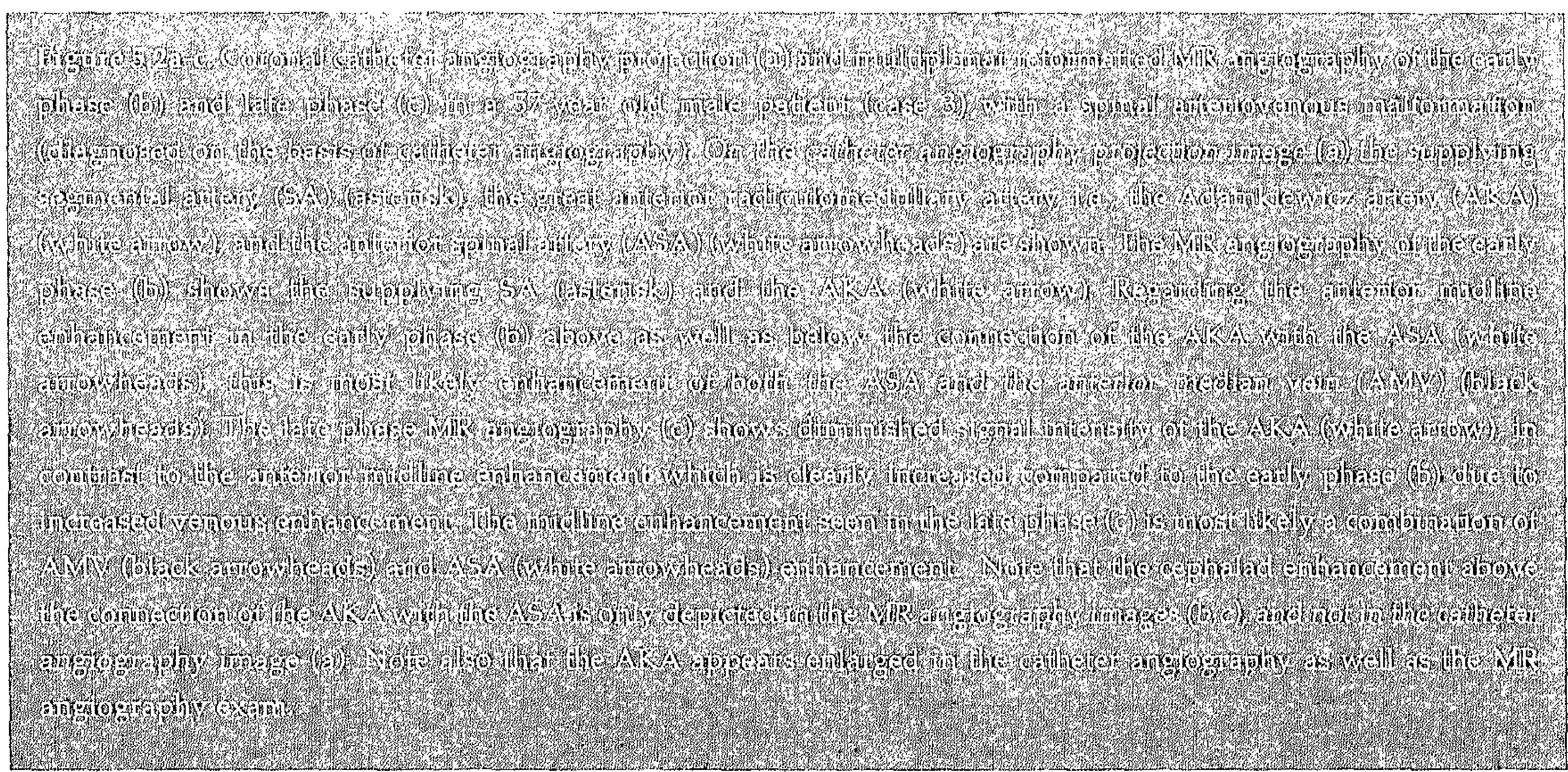




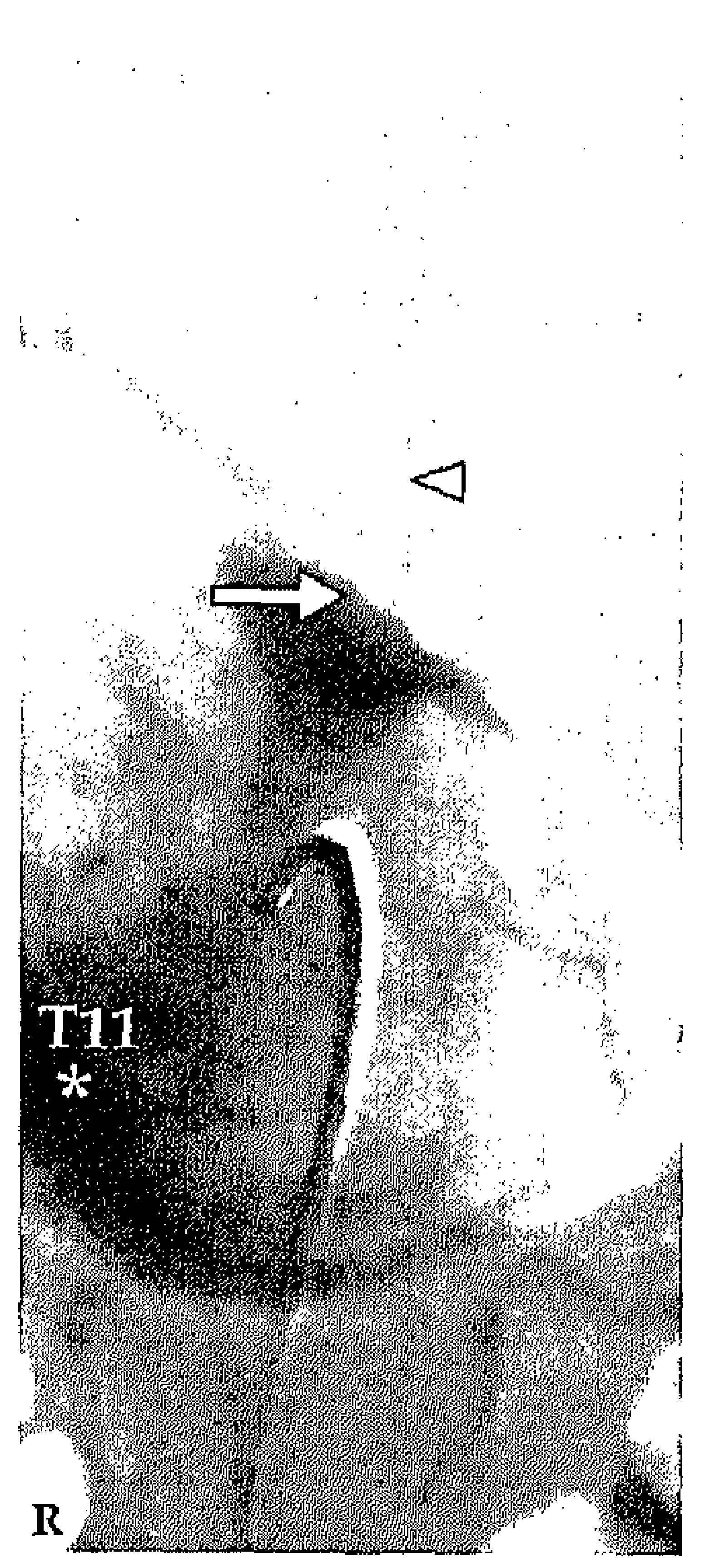

a

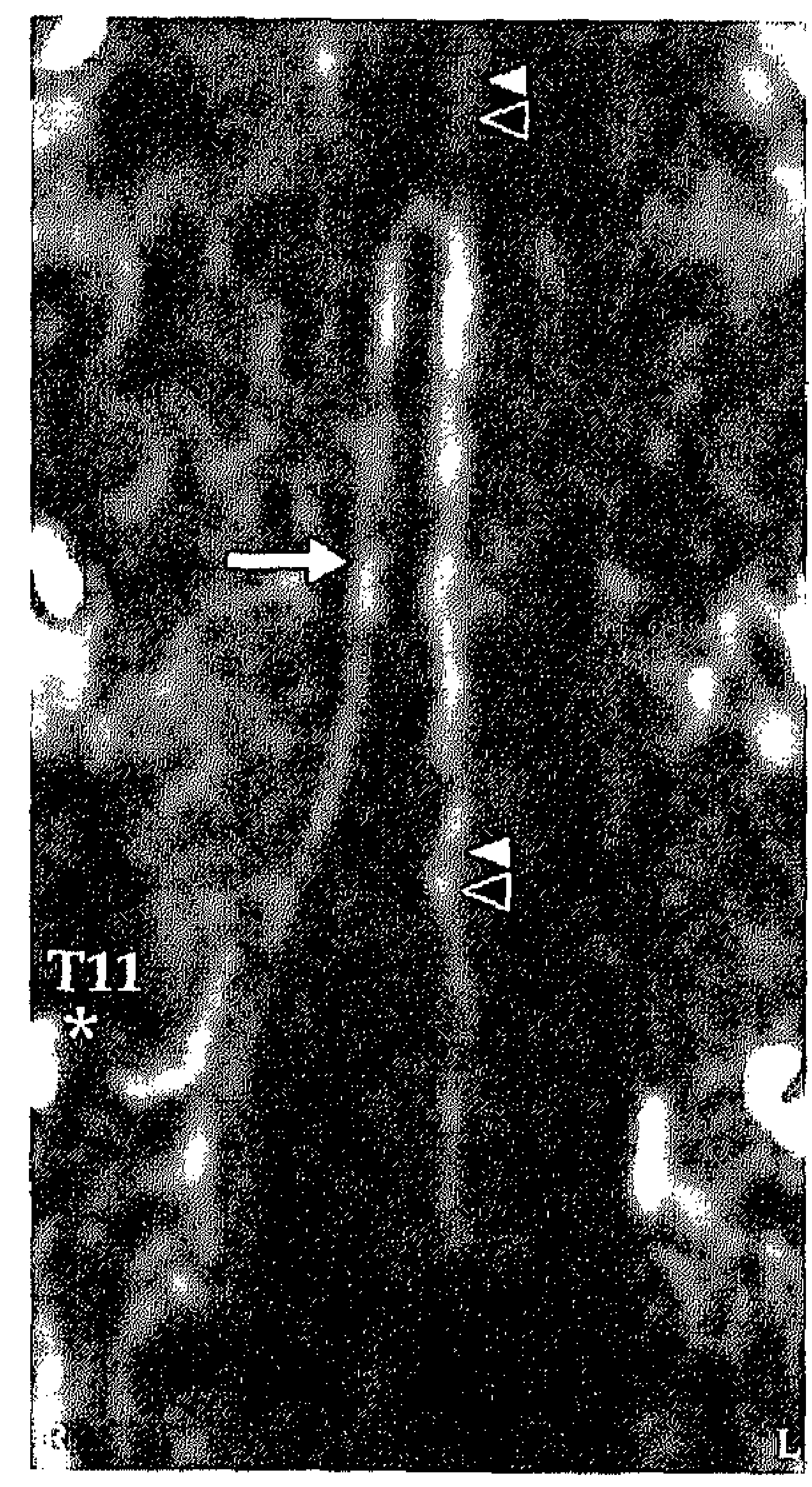

b

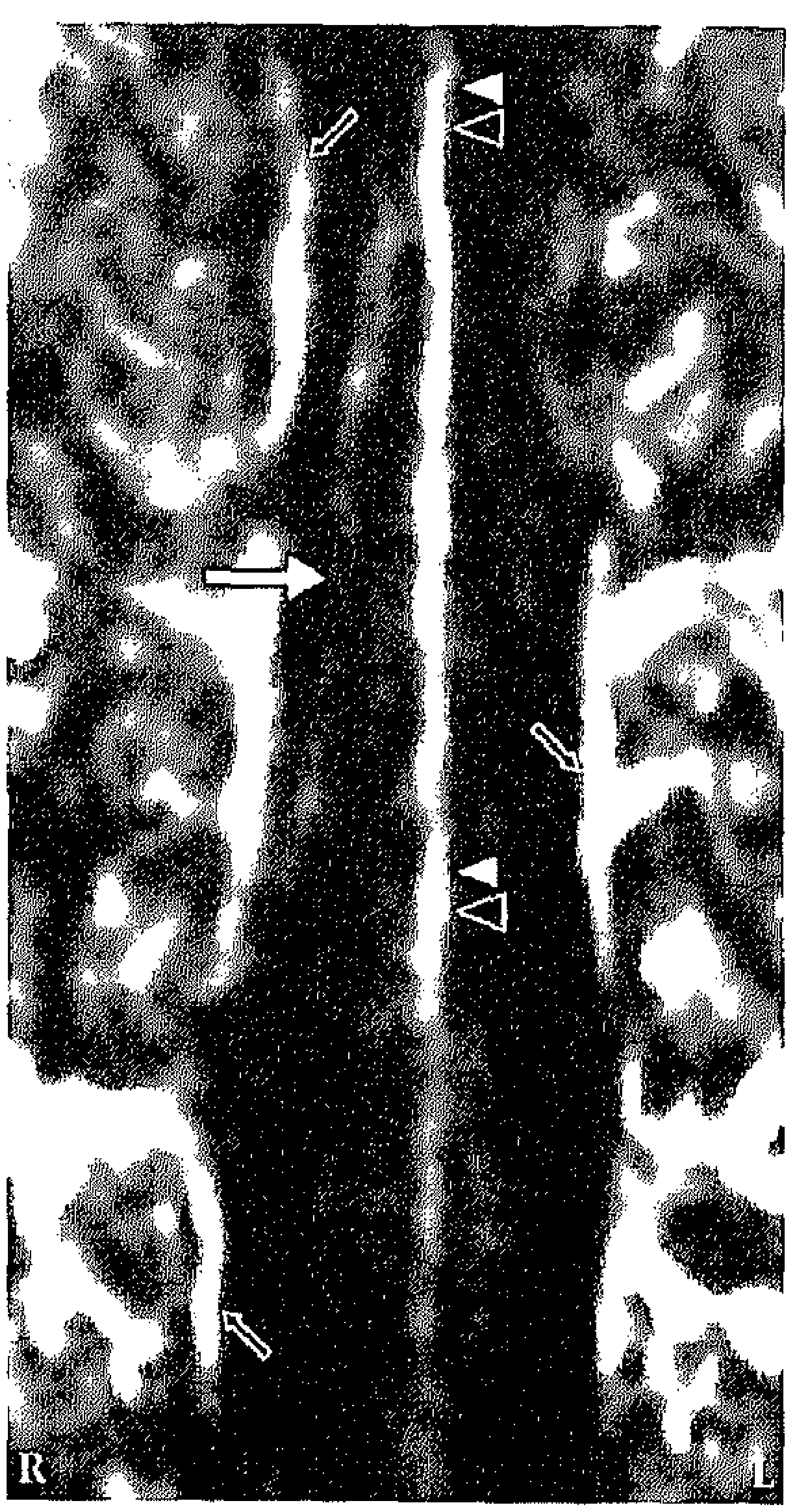

C

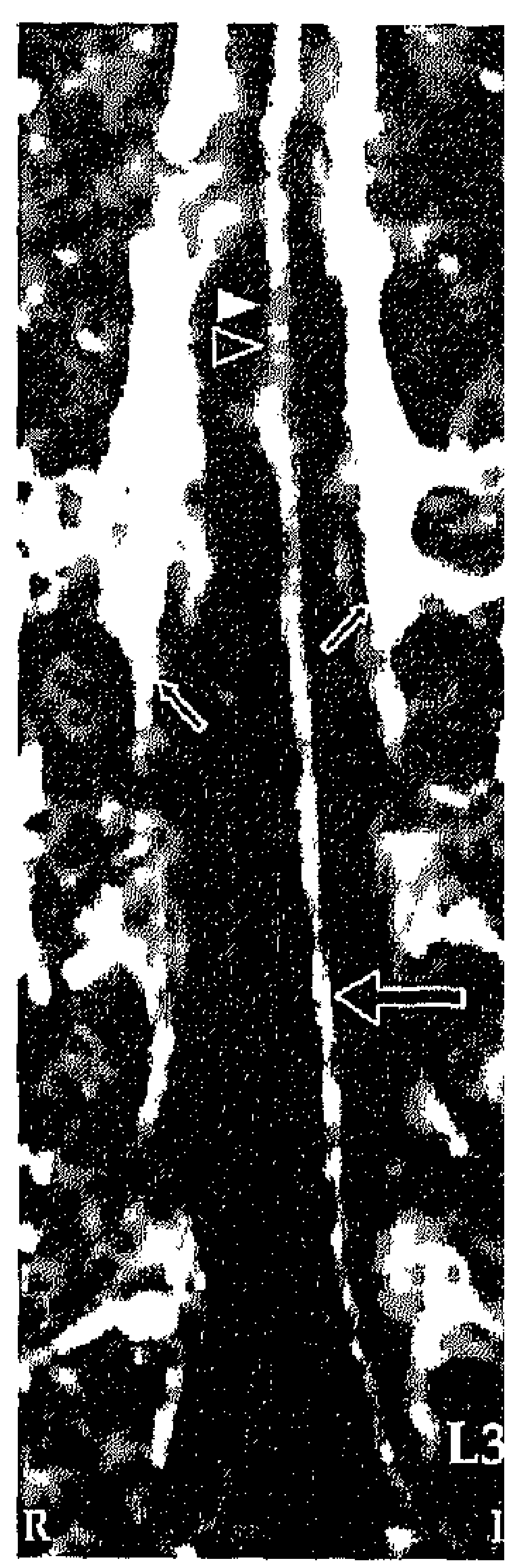

d

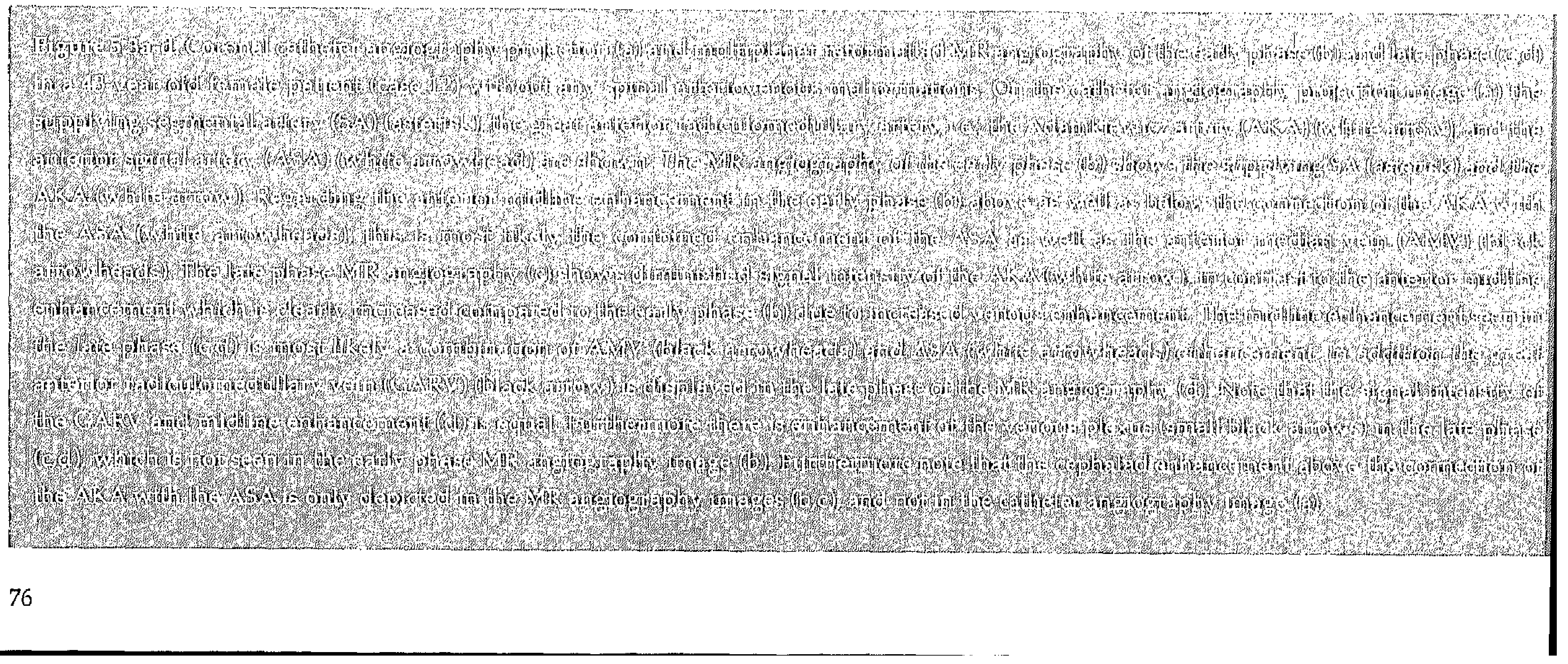




\section{Image analysis}

Postprocessing. The MR angiography images were postprocessed using curved multiplanar reformations (MPR). One creator determined the level and side of the AKA. In addition, this creator provided a single digital first phase MR angiography image in which the AKA was optimally displayed for the consensus comparison study with catheter angiography. Only one creator was used to prepare high-quality MR angiography images of the spinal vasculature for the comparison study. Localization of the AKA could be performed within 15 minutes. However, to optimally display the entire AKA from its origin of the posterior segmental artery (SA) to its connection and continuation to the ASA took 1-2 hours because the course of the AKA and its continuation are not situated in a single plane, and therefore (locally variably) curved MPR images had to be created. Catheter angiography images were printed on film.

Vessel identification. The following criteria were applied to identify the AKA, the segmental origin of the AKA, and the great anterior radiculomedullary vein (GARV) on the first and second phase MR angiography images: (i) an enhancing vessel observed in the first phase coursing through the intervertebral foramen toward the spinal cord, with a steep ascending course and connecting to the midline vessel on the anterior spinal cord surface, that did not enhance more intensely in the second phase was considered to be the AKA. (ii) If an enhanced vessel was observed in the first phase that coursed from the spinal cord toward an intervertebral foramen, with a descending course and connected to the midline vessel on the anterior spinal cord surface, that did not enhance less intensely in the second phase, or was observed only in the second phase, this vessel was considered to be the GARV. (iii) The segmental level of origin of the AKA and GARV was confirmed with the use of the T2weighted anatomic images. The intervertebral foramen at which the spinal branch of the posterior SA entered the spinal canal and continued as the AKA was determined as the level of origin of the AKA. For instance, in case the spinal branch entered between T12 and L1, it was considered to be the segmental level T12. (iv) If more enhancing vessels were observed in the first phase MR angiography images that originated from an intervertebral foramen between T9 and L2 and continued toward the midline vessel on the anterior spinal cord surface, the one with the largest (i.e., widest) diameter was considered to represent the AKA and arteries of smaller diameter (i.e., thinner) were considered to be other anterior radiculomedullary arteries (ARAs). Vascular pathology was also investigated by catheter and MR angiography but is outside the scope of the current article and will be reported elsewhere.

Image quality measurements. Three experienced neuroradiologists jointly scored the following aspects of the images of both MR and catheter angiography: (i) level, side, and similarity in configuration of the AKA, (ii) depiction of additional ARAs, and finally (iii) image quality regarding background homogeneity, vessel sharpness, continuity, conspicuity, and contrast on a relative 5-point scale. Interpretation of relative scores was as follows: -2 when catheter angiography was clearly superior to MR angiography; -1 when catheter angiography was marginally superior to MR angiography; 0 for equal appearance; +1 when MR angiography was marginally superior to catheter angiography; +2 when 


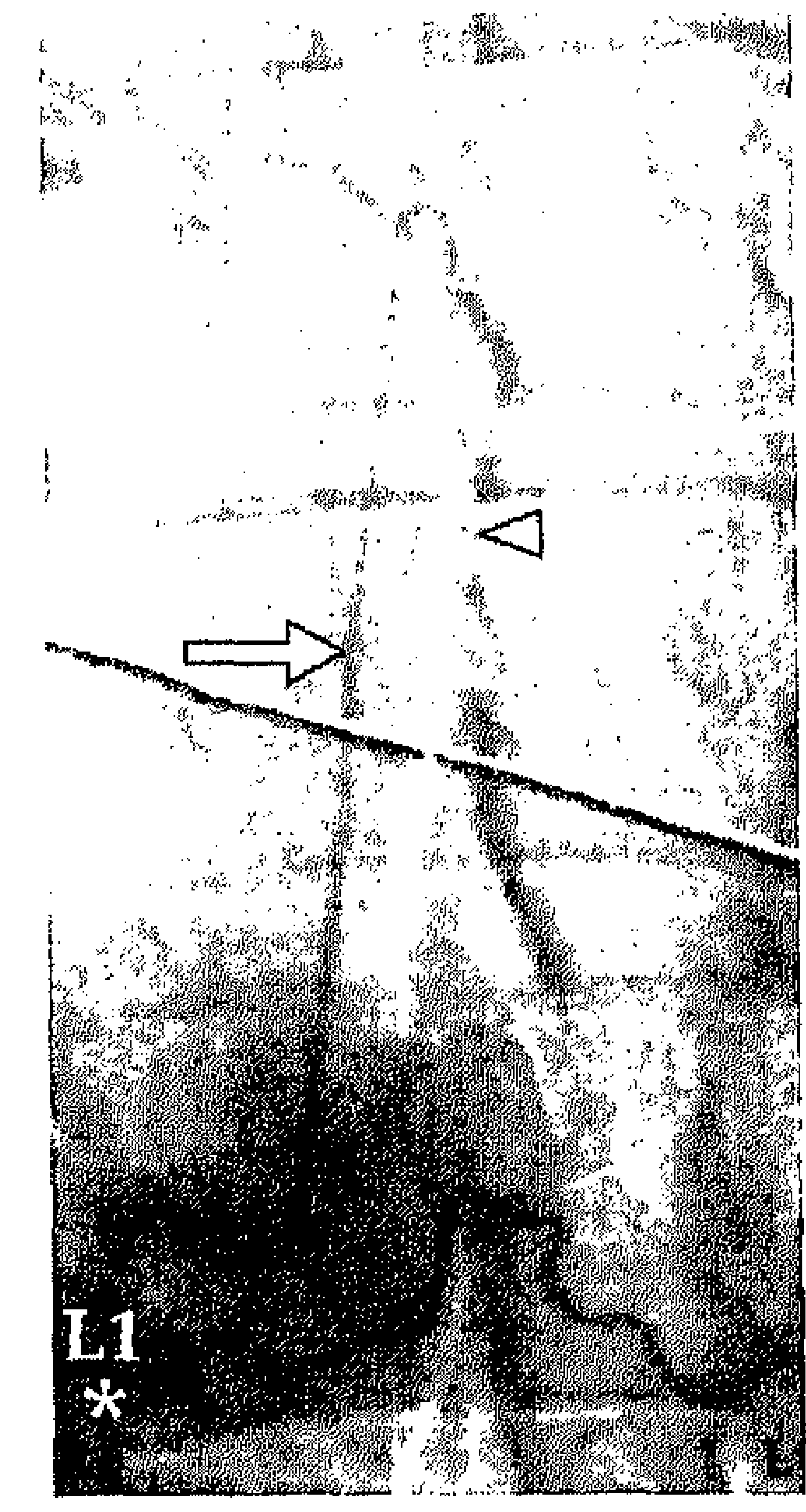

a

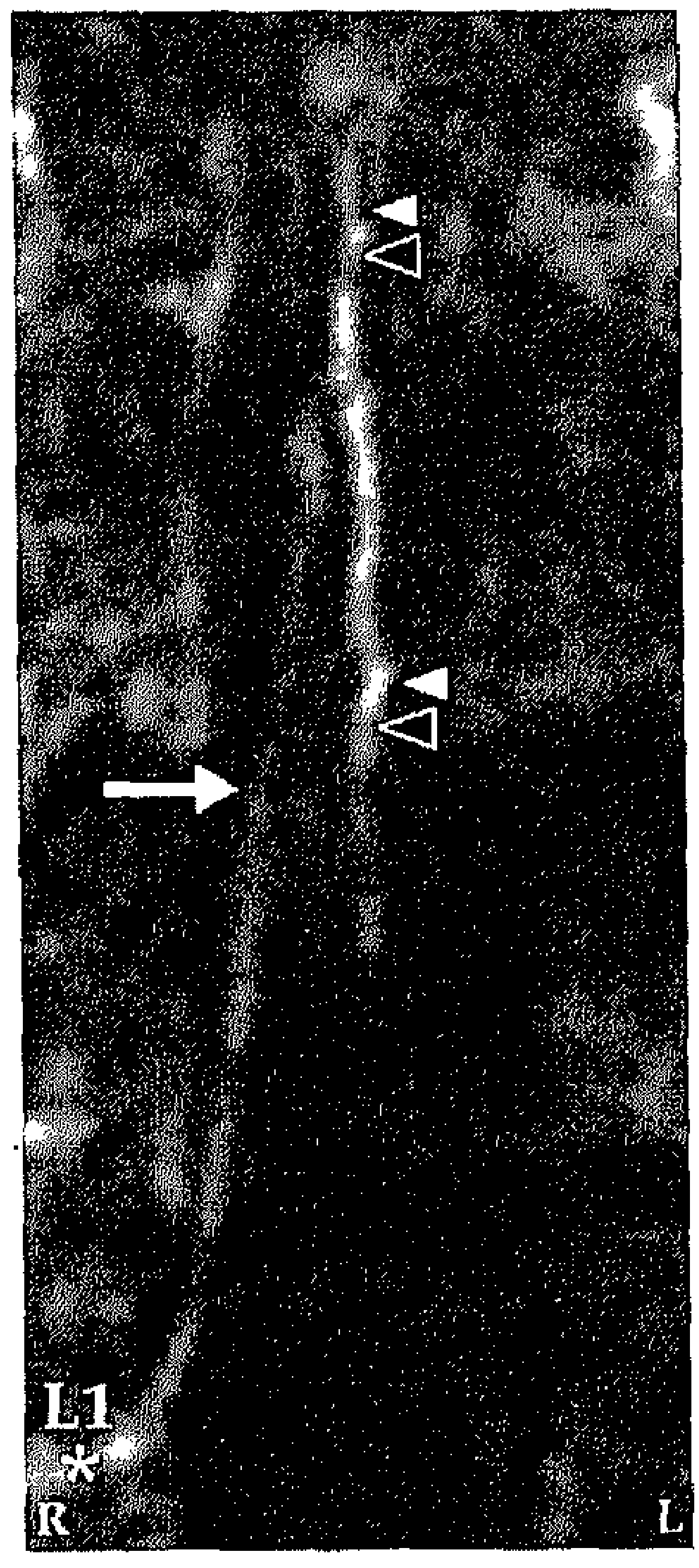

b

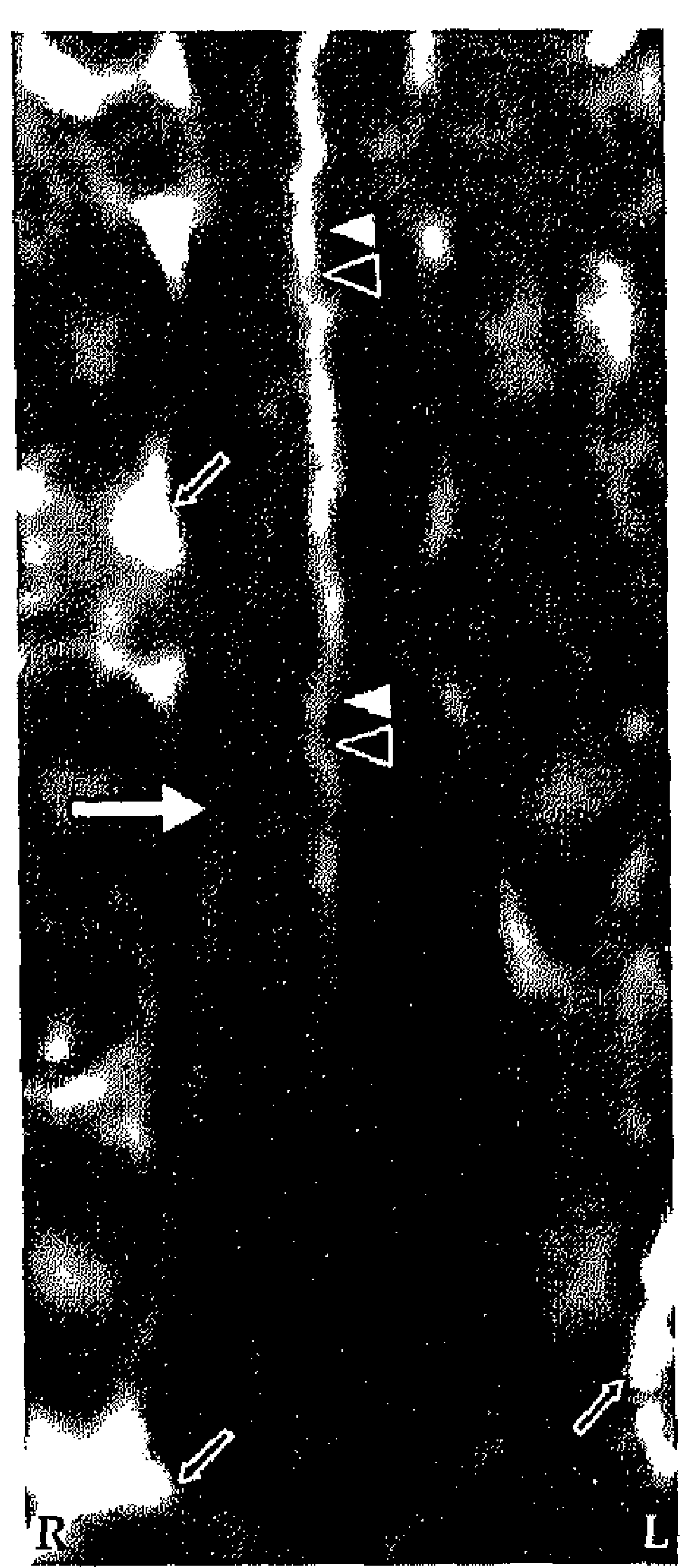

C

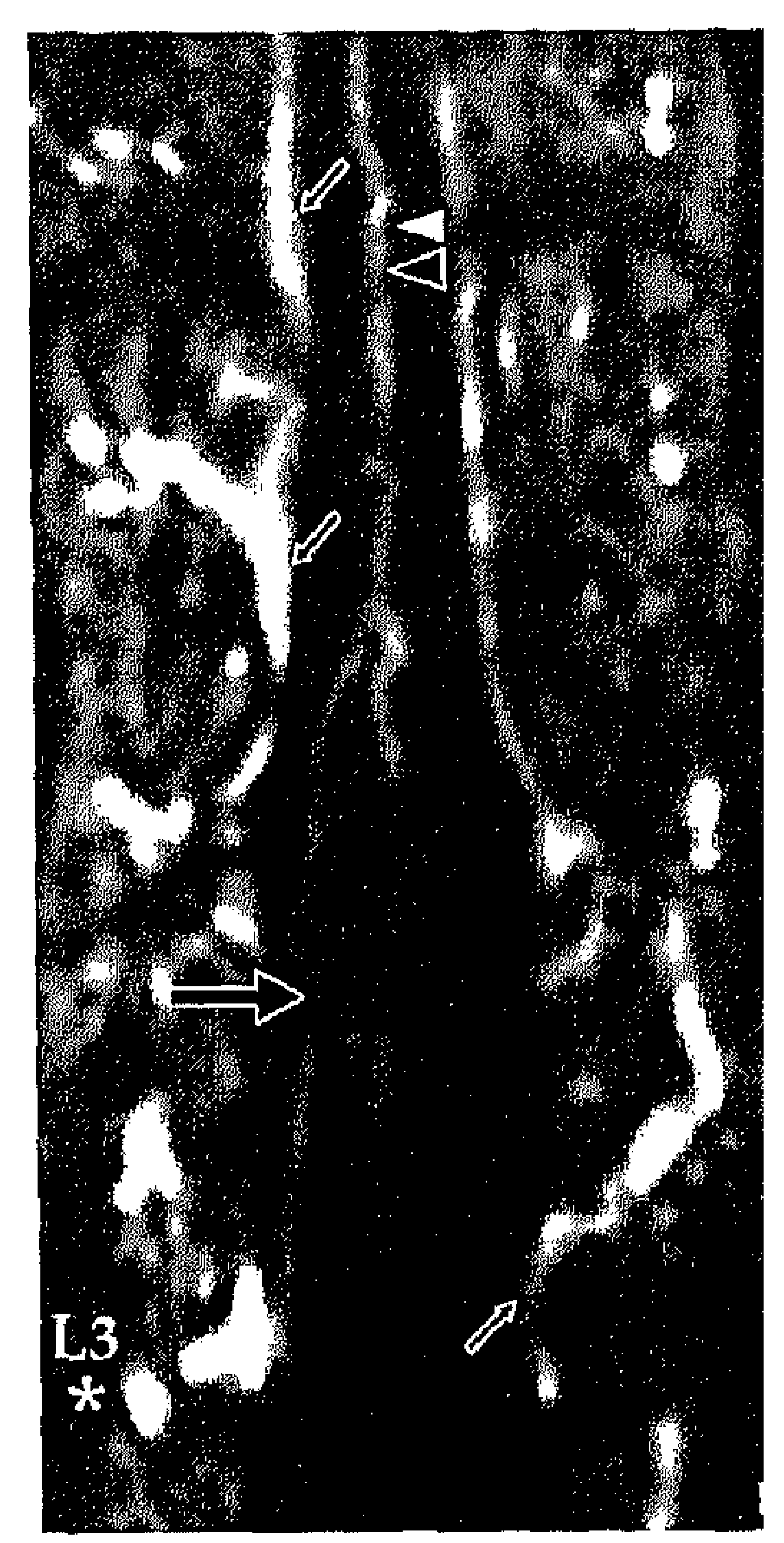

d

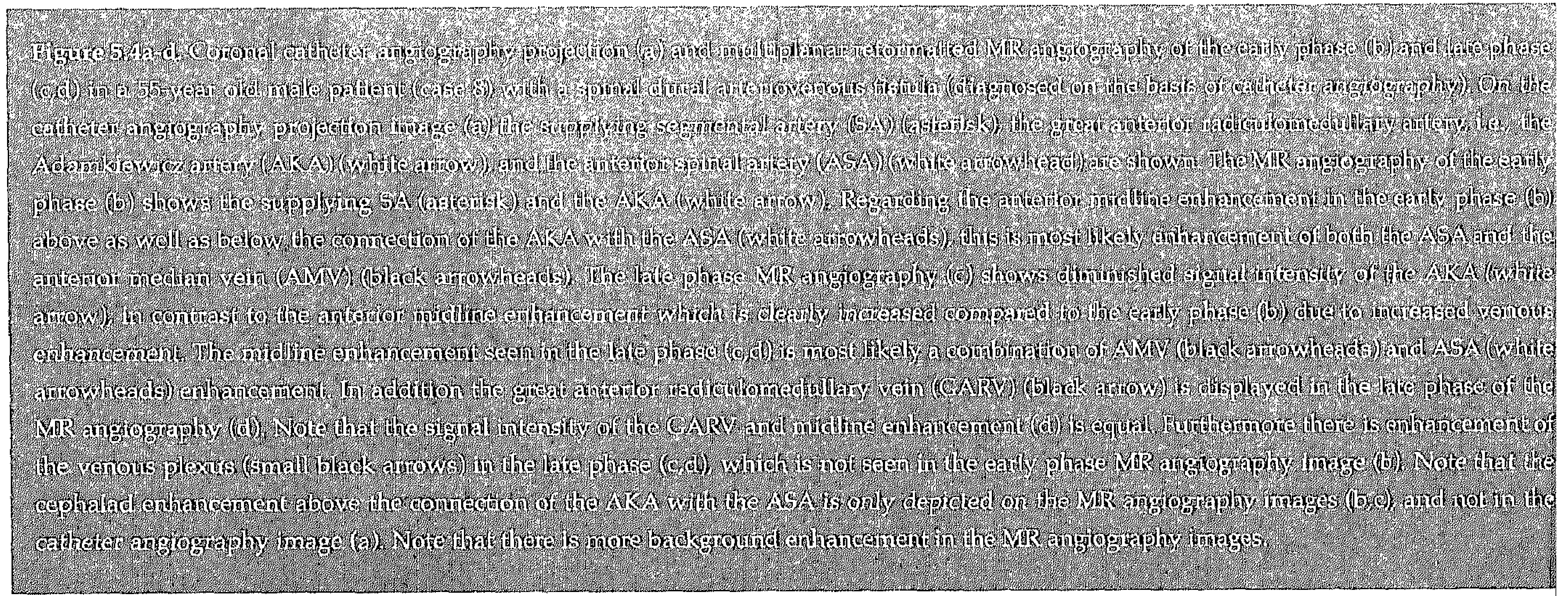


MR angiography was clearly superior to catheter angiography.

\section{Statistical analysis}

For statistical analysis of the image quality comparisons the (non-parametric) Wilcoxon paired sample test was used ${ }^{23}$. To this end, the absolute values of the relative scores were ranked for each image quality item. The ranks for the cases where MR angiography was better than catheter angiography (ie, positive relative scores) and where catheter angiography was better than MR angiography (ie, negative relative scores) were separately summed $\left(T_{+}\right.$and $T_{-}$, respectively) and nonparametrically (2-tailed) tested to be different from the critical value $\left(\mathrm{T}_{0.05,15}=25\right.$ for $n=15$ ) of the Wilcoxon $\mathrm{T}$ distribution. Statistical significance was inferred when the $P$-value obtained was less than 0.05 .

\section{RESULTS}

In all 15 patients, the AKA and its segmental origin were detected on both MR angiograhy phases and on catheter angiography (Figures 5.2-5.6). No adverse events occurred during or after any of the procedures. Table 5.1 summarizes the results of our patient group concerning lateralization and segmental origin of the AKA and GARV for MR angiography and catheter angiography.

The prospective localization and spatial configuration of the AKA as obtained by MR angiography was in agreement with the catheter angiography result in 14 of 15 cases (93\%) (Figures 5.2-5.5). In 1 case (case 11), there was disagreement between the catheter angiography (Figure 5.6a) and MR angiography regarding the vertebral level (not side) of the SA connecting to the AKA because of the tangled vascular pathology of multiple veins near the AKA (Figure 5.6b). The correct segmental origin could retrospectively be identified for this case (Figure 5.6c). The AKA derived from the left side in 10 patients (67\%) and from the right side in 5 patients (33\%). The AKA appeared enlarged in 2 patients (Figures 5.2 and 5.6) because of the underlying pathology (spinal arteriovenous malformation). An additional ARA was found in 2 cases by catheter angiography but not by MR angiography (Table 5.1).

In the second phase images, the GARV could be detected and localized in 13 of 15 patients (Figures $5.3 \mathrm{~d}, 5.4 \mathrm{~d}$, and $5.5 \mathrm{~d}$ ). This was in contrast to the catheter angiography, where the GARV was not detected in any of the cases. Based on the localization of the GARV from the second phase MR angiography images, retrospectively in 4 of 15 cases, the GARV was faintly visible in the first phase images.

\section{Image quality measurements}

Comparison of image quality between MR and catheter angiography (Table 5.2) revealed that catheter angiography was superior to $M R$ angiography $(P<0.001)$ concerning vessel continuity, sharpness, and background homogeneity. Overall vessel conspicuity and contrast did not differ $(P>0.05)$. 


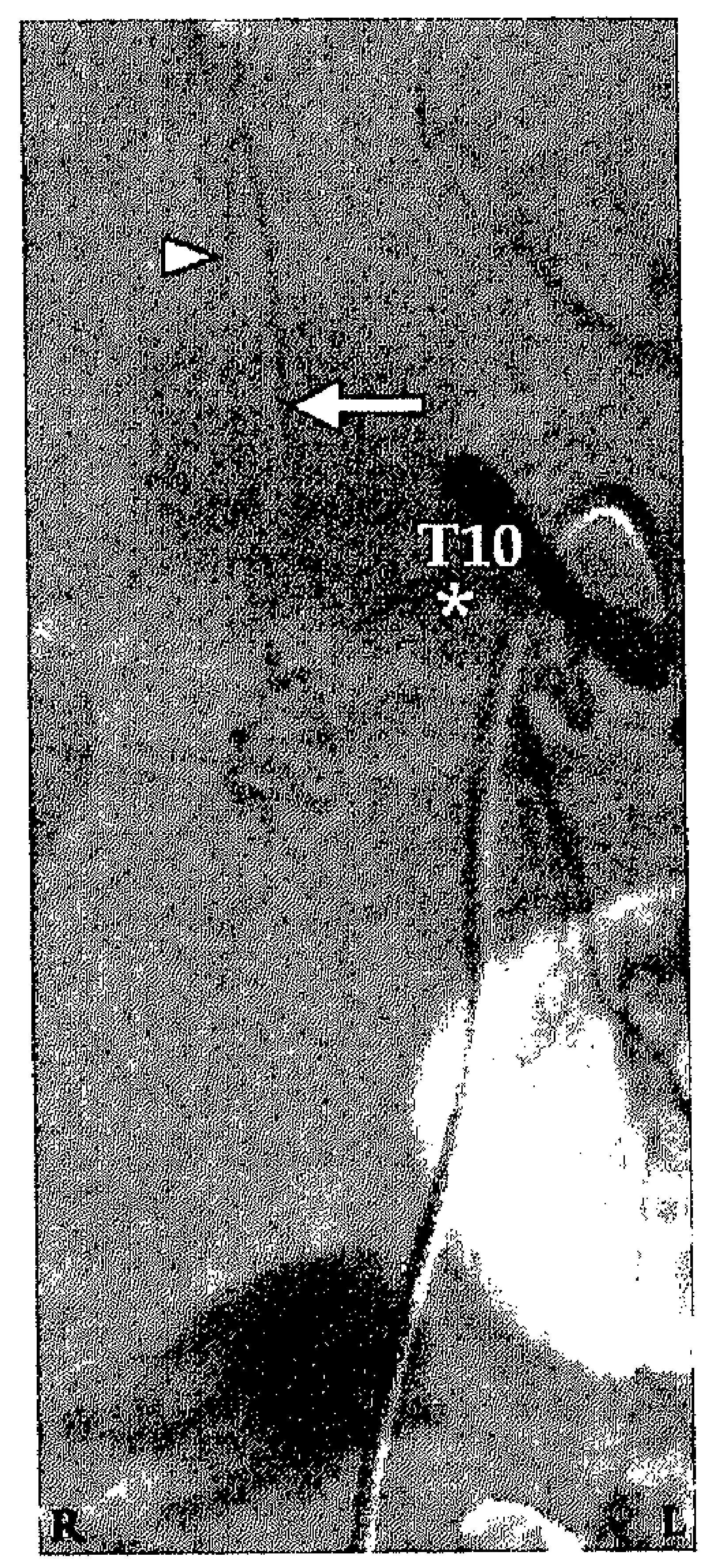

a

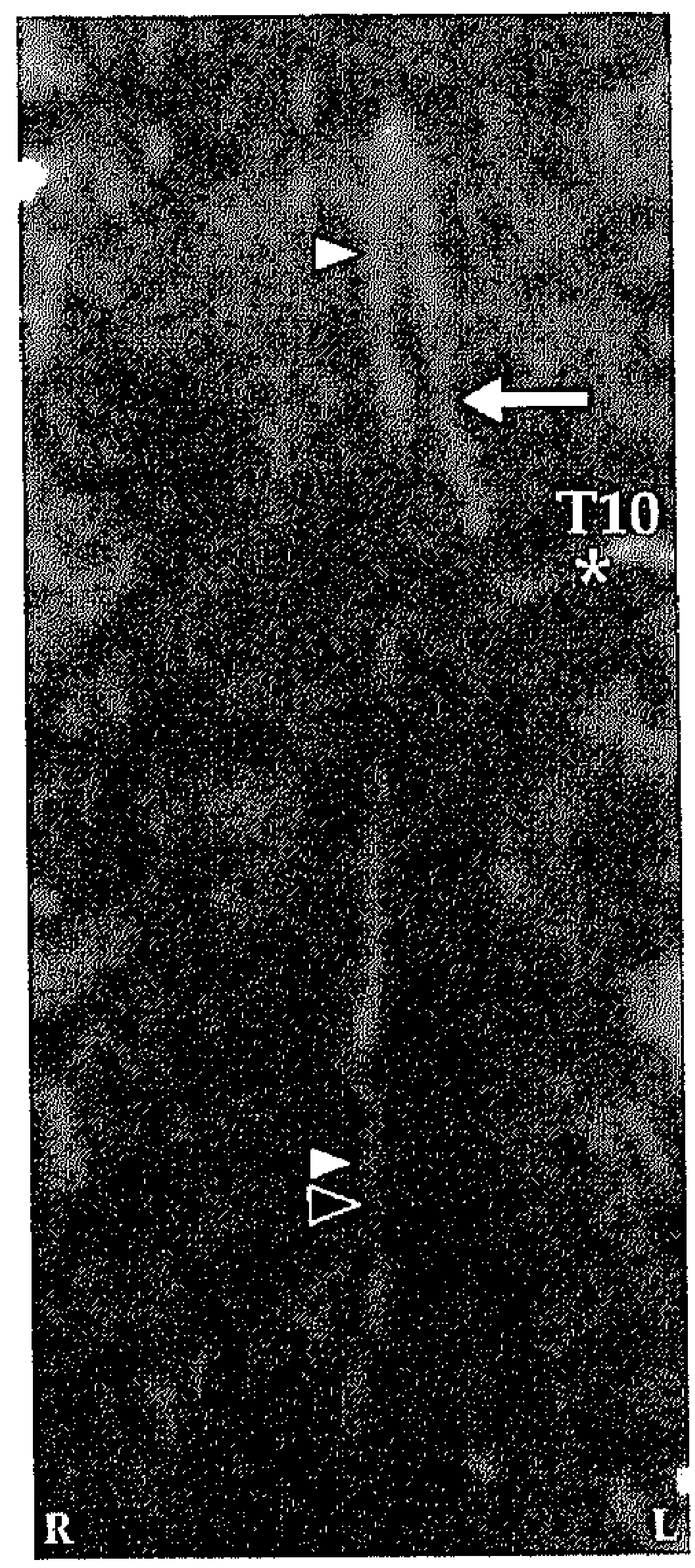

b

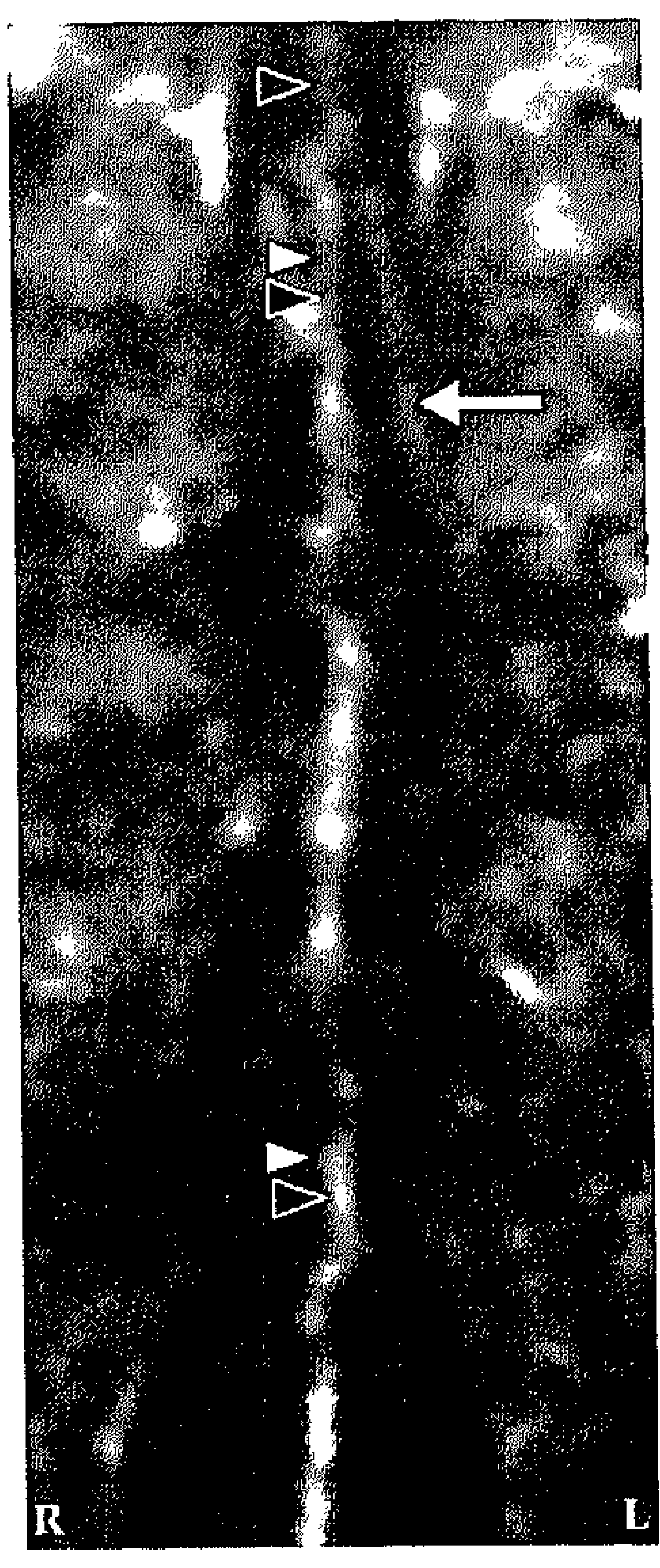

C

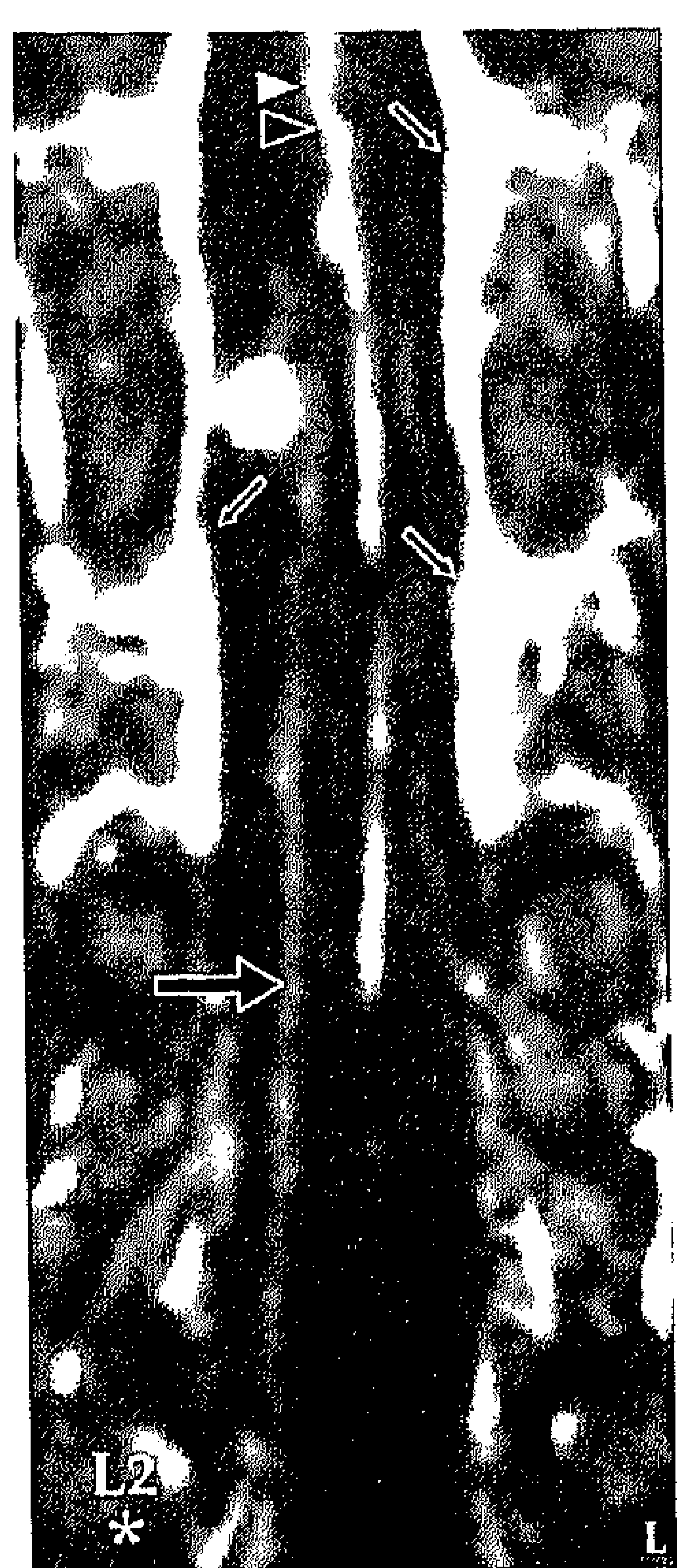

d

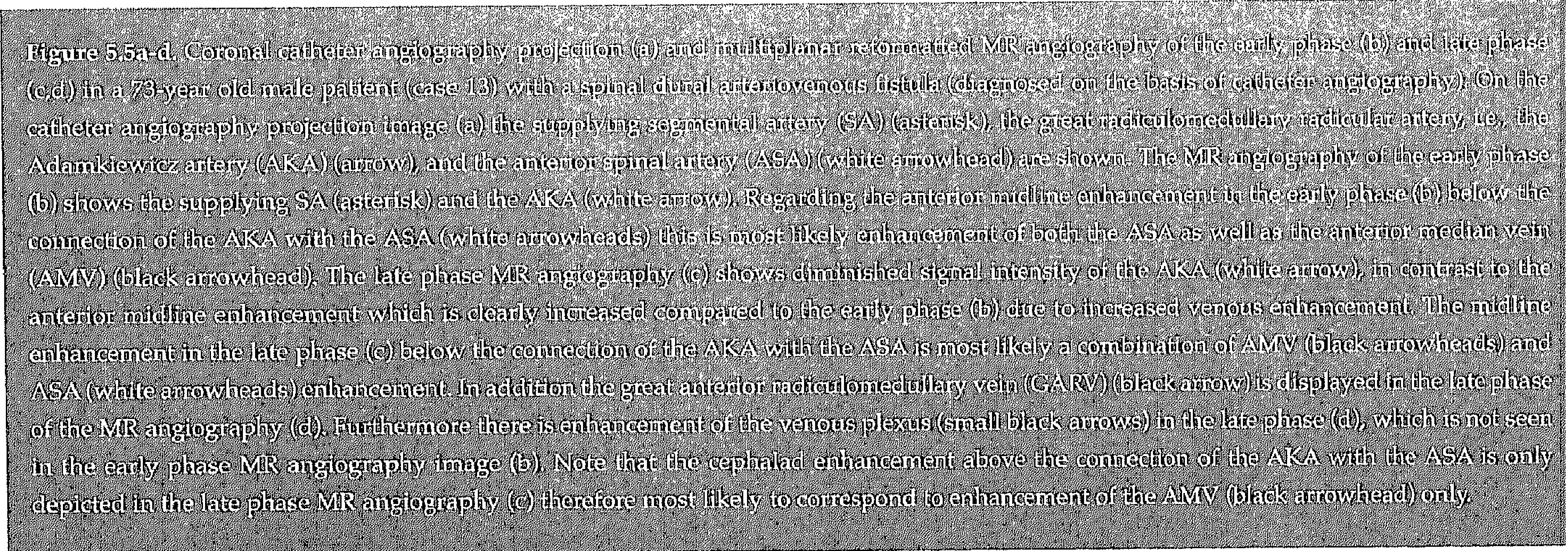



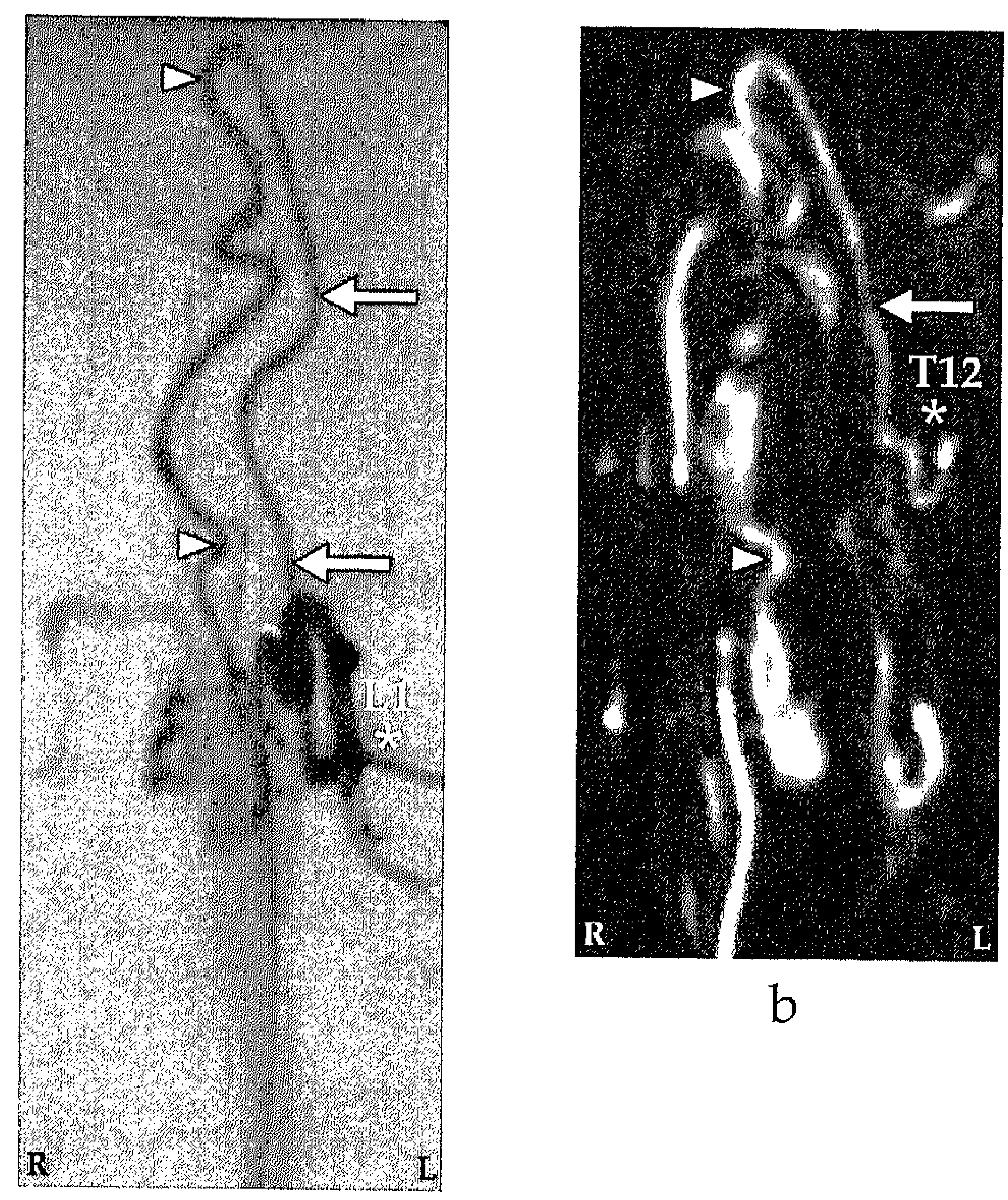

b

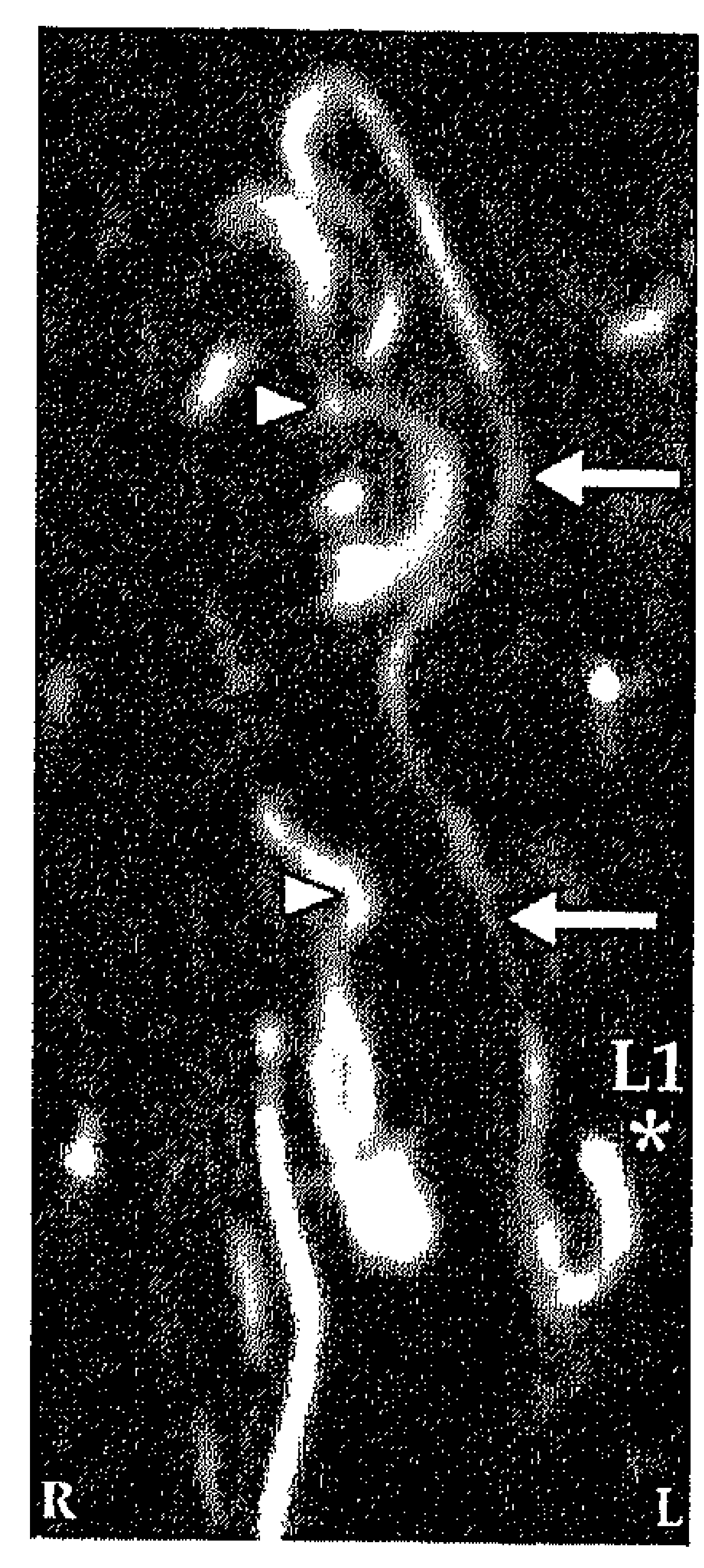

C

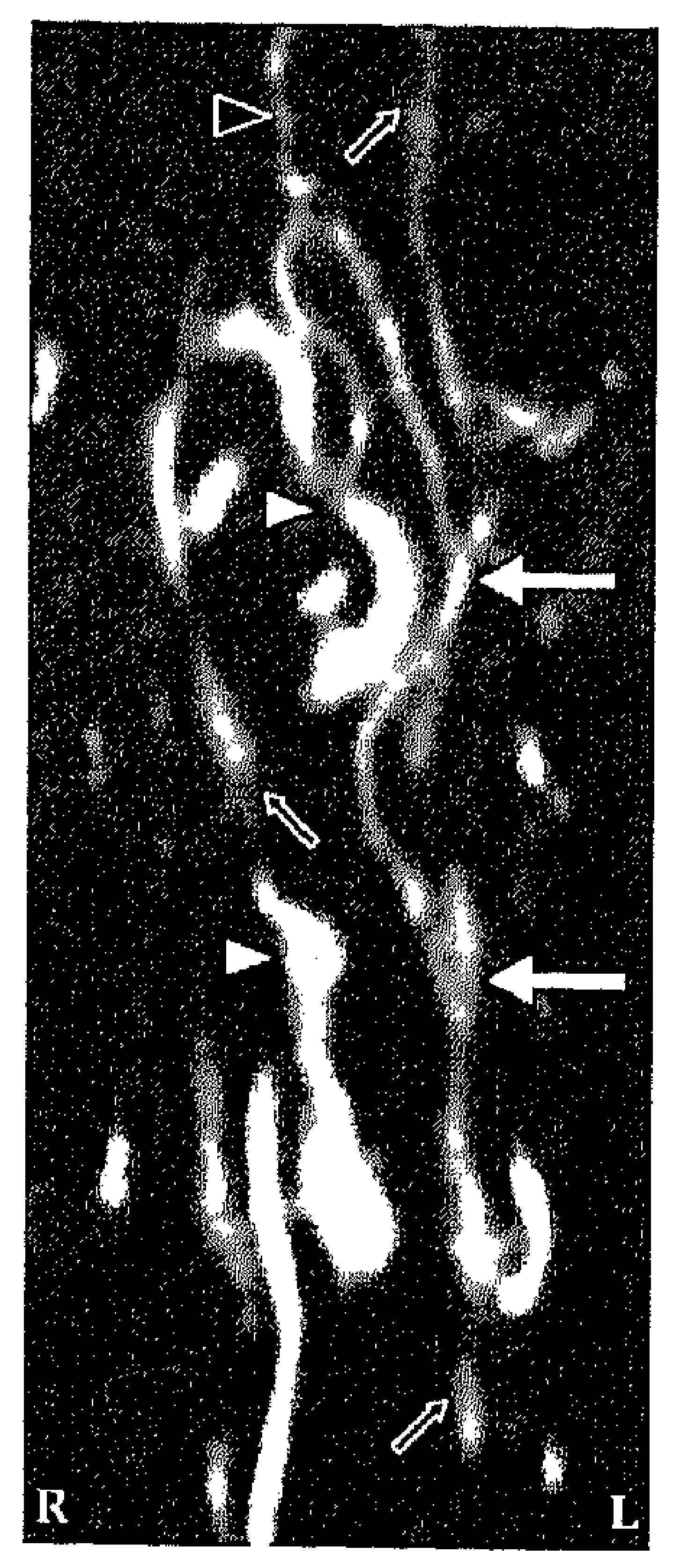

d

a

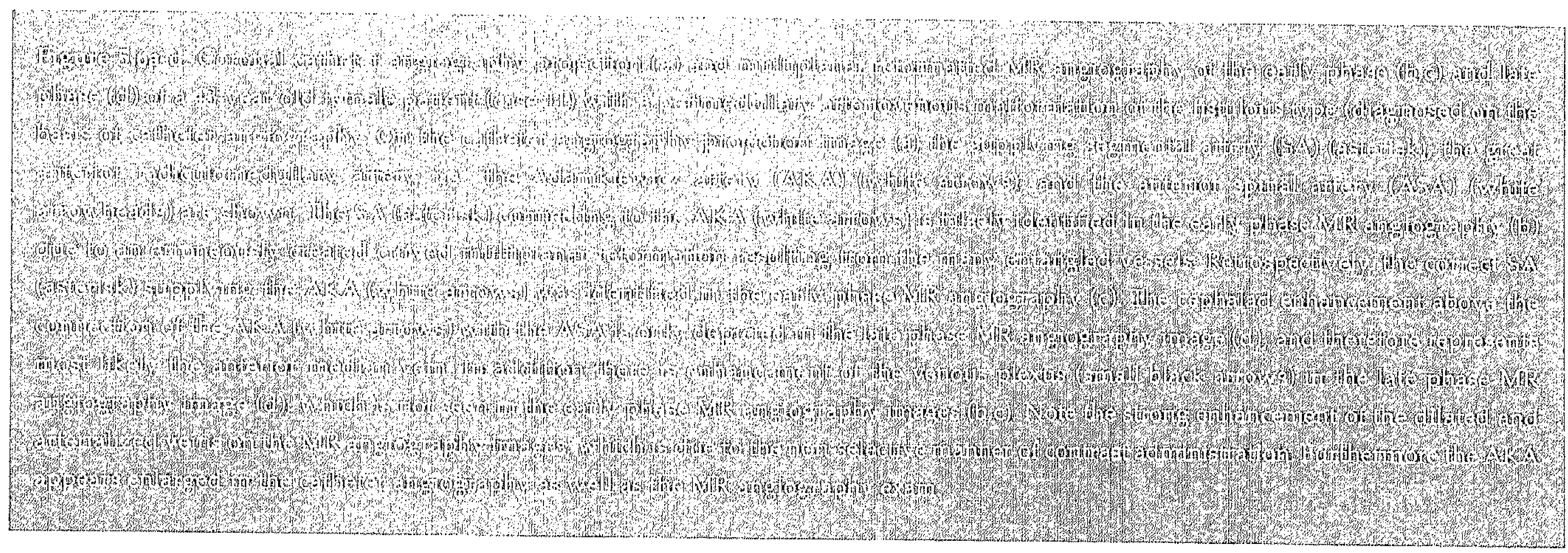


Table 5.2. Comparison of image quality between MR angiography and catheter angiography.

$\begin{array}{lccccc} & \begin{array}{c}\text { Background } \\ \text { homogeneity }\end{array} & \begin{array}{c}\text { Vessel } \\ \text { sharpness }\end{array} & \begin{array}{c}\text { Vessel } \\ \text { continuity }\end{array} & \begin{array}{c}\text { Vessel } \\ \text { conspicuity }\end{array} & \text { Contrast } \\ \text { Relative score } & -1.5 \pm 0.8 & -1.6 \pm 1.1 & -1.5 \pm 0.6 & -0.7 \pm 1.3 & 0.3 \pm 1.4 \\ \mathrm{~T}+/ \mathrm{T} \text { - } & 0 / 114 & 0 / 120 & 0 / 119 & 25.5 / 96.5 & 71 / 34 \\ P \text {-value } & <0.001 & <0.001 & <0.001 & >0.05, \text { n.s. } & >0.05 \text {, n.s. }\end{array}$

A negative (positive) score indicates that catheter angiography performs better (worse) than MR angiography.

Notation: mean relative score \pm one standard deviation; $T_{+} / T_{-}$: sum of ranks with a plus $/$minus sign; $n . s .=$ non significant

\section{DISCUSSION}

In this study, two-phase MR angiography was compared with catheter angiography for the detection and localization of the great anterior radiculomedullary artery (i.e., the AKA) and to differentiate it from the GARV. The two most important aspects in MR angiography of the anterior superficial spinal cord arteries that determine the image result are spatial and temporal resolution. Achieving a high spatial resolution, which is required for visualization of the submillimeter-sized arteries $(<1.0 \mathrm{~mm})$, is hampered by the fact that a large field of view has to be used. Furthermore, when no clear temporal separation between the anterior superficial spinal cord arteries and veins is possible, no definitive judgment can be made whether a depicted vascular structure on the anterior spinal cord surface is arterial or venous, because the configuration and midline localization of the anterior superficial spinal cord veins and arteries is quite similar. The temporal resolution that has to be achieved is on the order of (or less than) 10 seconds, because this is the estimated arteriovenous window of the normal spinal circulation time that has been reported 15 .

Until now, several MR angiography techniques have been attempted to visualize the anterior superficial spinal cord arteries. The first techniques used were blood flowdependent, such as 3D phase conventional angiography (3D PCA) $11,12,14$ and 3D contrastenhanced time-of-flight imaging (3D CETOF) $9,11,24$. With 3D PCA, only an enlarged AKA could be depicted in the pathologic situation of increased flow as occurring in arteriovenous malformations. However, no separation could be achieved between anterior superficial spinal cord arteries and veins. The greatest disadvantage of the 3D CE-TOF technique is the long acquisition time required ( 10 minutes), resulting in substantial venous as well as background enhancement ${ }^{11,24}$. Despite the use of a contrast medium in 3D CE-TOF imaging, the anterior superficial spinal cord arteries were poorly resolved $9,11,24$ because the larger veins may overshadow smaller neighbouring arteries. To achieve a better arterial visualization without venous enhancement, first-pass MR imaging featuring a rapid injection of contrast medium and a 
high-speed sophisticated acquisition sequence has been proposed $8,10,13,17,25$. Using centric kspace sampling, Willinek et al ${ }^{26}$ have shown that it is possible to achieve an effective temporal resolution of 5-10 seconds, though the actual total acquisition duration may be quite longer. In the current study, we have used the centric ordered k-space filling to enable an effective temporal resolution of 5-10 seconds that is devoted to the arterial phase. The remaining 31 seconds of the MR amgiography first phase sequence, in which both arteries and veins are both filled with contrast agent, were used to increase spatial resolution (e.g., delineation and sharpness of the enhanced vessels).

Although the GARV was already enhanced in the first phase MR image in 4 of the 15 cases, we believe that the two-phase MR angiography approach enables a reliable differentiation between the AKA and GARV as the signal intensity of the GARV increases and that of the AKA decreases in the second phase compared with the first phase MR angiography images. Therefore, bolus arrival timed (i.e., first-pass) imaging using centric ordered $\mathrm{k}$-space filling is, in our experience, the best technique to obtain the necessary temporal resolution for arteriovenous separation of the AKA from the GARV. However, whether the current temporal resolution is sufficient to separate the ASA from the anterior median vein (AMV) remains doubtful, especially in cases with an aberrant spinal cord circulation. A limitation of the current study is therefore that the inclusion of patients with vascular spinal cord malformations complicates the separate depiction of the ASA and the AMV.

In addition, with non-centric ordered $k$ space filling, separation of arteries from veins seems possible as proved by Yamada et al6,7.
They were the first to report on the visualization of the AKA using a MR angiography technique with two dynamic phases and were able to distinguish the AKA from the GARV in some cases. However, the detection rate of the AKA in this study of 26 patients was only $69 \%$, which is lower than in the current study, whereas catheter angiography validation was performed in only 3 cases.

The present comparative study has shown that though the image quality of $M R$ angiography was found to be inferior to that of catheter angiography, MR angiography offers sufficient image (artery to background) contrast to consistently detect the AKA in patients with and without SAVMs. In our patient population, there were 2 spinal arteriovenous malformations (SAVMs), 8 spinal dural arteriovenous fistulas (SDAVFs), 1 hemangioblastoma, and 1 spinal subarachnoidal hemorrhage. Three patients had no pathologic conditions of the spinal vasculature. A SAVM may affect the spinal circulation time. The arteriovenous window in a SAVM may be shortened, in contrast to an SDAVF, where it may be prolonged compared with normal spinal circulation ${ }^{27}$. Therefore, knowledge of the underlying circulation time seems to be important because this may influence the imaging time, especially for the filling of the k-space center. However, the exact period for shortening or prolongation of spinal cord circulation is unknown. Therefore, all patients were imaged with the same MR imaging protocol and the same criterion (complete aortic filling) for the scan delay. Regarding the possible suboptimal timings of the spinal cord artery filling and center of kspace acquisition, the presence of a pathologic vascular condition (except for one case) obviously did not impair AKA identification. 
In one case, we obtained a mismatch of 1 vertebral level (not side) because of the tangle of multiple veins close to the AKA. The arteriovenous window for the SAVM apparently was too short to be separated by the MR acquisition technique used. However, as reported previously by others 28 , the subsequent catheter angiography examination would still have been limited in procedure time and radiation dose, because adjacent levels are usually examined for pathologic conditions as well.

On the anterior spinal cord surface, cephalad enhancement above the connection of the AKA was found more often in first phase MR angiography than with early phase catheter angiography. Although this might be the ascending continuation of the AKA into the ASA, it is more likely to be venous enhancement of the AMV. Nevertheless, contribution of the ASA cannot be ruled out completely, because the ASA above the connection with the AKA may be supplied by an ARA derived from a higher thoracic or even cervical level. Moreover, the cephalad enhancement above the connection of the AKA might indeed in some instances be the ascending part of the AKA, because in two catheter angiography examinations, cephalad enhancement was depicted.

Catheter angiography achieves a spatial resolution of approximately $0.2 \mathrm{~mm}$, which is not yet feasible with clinical MR angiography. This superior spatial resolution was apparent in two cases in which an additional ARA was found by catheter angiography but not with MR angiography. Anatomic literature has reported that the AKA is the largest radiculomedullary artery (diameter, $0.5-1.0 \mathrm{~mm}$ ). In a previous study, it was shown that vessels with diameters less than or equal to $0.3 \mathrm{~mm}$ cannot be visualized ${ }^{8}$. The additional ARAs that were found by catheter angiography (at $\mathrm{T} 4$ and $\mathrm{T} 3$ in cases 1 and 5 , respectively) are therefore probably $0.3 \mathrm{~mm}$ or less in diameter. Moreover, the middle and upper thoracic part of the ASA has been described as difficult to image $^{8}$ because this part may suffer quite substantially from artifacts related to cardiac motion. The high spatial resolution for catheter angiography is also demonstrated by the fact that vessel continuity and sharpness were significantly better when using this technique. Background inhomogeneity (e.g., vertebral lipid) was stronger for $M R$ angiography according to the observers. Catheter angiography suffers less from this phenomenon because selective arterial injection and a subtraction technique was used to reduce background signal intensity (e.g., bone). Vessel conspicuity and contrast for the AKA were found to be equal (or even better) for MR angiography, but the differences were not statistically significant. The two most important aspects that determine whether one can depict an anterior superficial spinal cord artery by MR angiography are continuity and contrast. As explained above, the high spatial resolution obtained by catheter angiography allows better visualization of vessel continuity. However, contrast was found to be slightly better on MR angiography images, which is probably due to the partial-volume effect, which made the anterior superficial spinal cord arteries appear wider. So, although most included patients had a spinal vascular abnormality, hampering AKA depiction, especially in case of an SDAVF with an aberrant spinal cord circulation, $M R$ angiography was able to correctly localize the AKA in $100 \%$ of the cases. 
Visualization of the AKA by MR imaging can be of use in several clinical situations, such as preoperative work-up for thoracoabdominal aortic aneurysm surgery 19,20 , thoracoscopic spinal hernia surgery, and transarterial embolization of vertebral metastases ${ }^{29}$, and may reduce risk for neurologic complications related to the lower spinal cord. In addition, when diagnosing SAVMs, such as in SDAVF or SAVM, AKA localization by MR angiography may have an additional precautionary contribution in that the AKA may derive from the same segmental artery as the SAVM. Moreover, a number of reports $10,17,25$ have demonstrated that the feeding segmental artery of an SDAVF can be localized with the use of MR angiography.

To incorporate the described MR angiography protocol in routine clinical practice, dedicated image postprocessing is required, and in-depth knowledge of vascular spinal cord anatomy is essential. This especially applies to cases with vascular abnormalities of the spinal cord vasculature in which the vessels may obscure the depiction of the anterior superficial spinal vasculature.

In conclusion, a non-invasive diagnostic imaging technique for visualization of the anterior superficial spinal cord arteries is of interest in several clinical situations. By validating $M R$ angiography against catheter angiography, two-phase MR angiography was demonstrated to reliably detect and localize the AKA of the thoracolumbar spinal cord in patients with and without pathologic conditions of the spinal cord vasculature.

\section{Acknowledgement}

We thank B.C. Bowen for kindly providing the photograph in Figure 5.1. 


\section{REFERENCES}

1. Kudo K, Terae S, Asano T, et al. Anterior spinal artery and artery of Adamkiewicz detected by using multidetector row CT. AINR Am I Neuroradiol 2003; 24:13-7.

2. Takase K, Sawamura Y, Igarashi $K$, et al. Demonstration of the artery of Adamkiewicz at multidetector row helical CT. Radiology 2002;223:39-45.

3. Yoshioka $\mathrm{K}$, Niinuma $\mathrm{H}$, Ohira $\mathrm{A}_{\text {, et al. } \mathrm{MR}}$ angiography and $\mathrm{CT}$ angiography of the artery of Adamkiewicz: noninvasive preoperative assessment of thoracoabdominal aortic aneurysm. Radiograplics 2003;23:1215-25

4. Kawaharada N, Morishita K, Fukada J, et al. Thoracoabdominal or descending aortic aneurysm repair after preoperative demonstration of the Adamkiewicz artery by magnetic resonance angiography. Eur / Cardiothorac Surg 2002;21:970-4.

5. Kawaharada N, Morishita K, Hyodoh $\mathrm{H}$, et al. Magnetic resonance angiographic localization of the artery of Adamkiewicz for spinal cord blood supply. Ann Thorac Surg 2004;78:846-51; discussion 851-2.

6. Yamada N, Okita Y, Minatoya K, et al. Preoperative demonstration of the Adamkiewicz artery by magnetic resonance angiography in patients with descending or thoracoabdominal aortic aneurysms. Eur J Cardiothorac Surg 2000;18:104-11.

7. Yamada N, Takamiya M, Kuribayashi S, et al. MRA of the Adamkiewicz artery: a preoperative study for thoracic aortic aneurysm. I Comput Assist Tomogr 2000;24:362-8.

8. Nijenhuis RJ, Leiner T, Cornips EM, et al. Spinal cord feeding arteries at MR angiography for thoracoscopic spinal surgery: feasibility study and implications for surgical approach. Radiology 2004;233:541-7.

9. Bowen $B C$, DePrima $S$, Pattany PM, et al. MR angiography of normal intradural vessels of the thoracolumbar spine. AJNR Am I Neuroradiol 1996;17:483-94.

10. Farb RI, Kim JK, Willinsky RA, et al. Spinal dural arteriovenous fistula localization with a technique of first-pass gadolinium-enhanced $M R$ angiography: initial experience. Radiology 2002;222:843-50.

11. Mascalchi $M$, Bianchi $M C$, Quilici $N$, et al. $M R$ angiography of spinal vascular malformations. AJNR Am J Neuroradiol 1995;16:289-97.

12. Mascalchi $M$, Quilici N, Ferrito $G$, et al. Identification of the feeding arteries of spinal vascular lesions via phase-contrast MR angiography with threedimensional acquisition and phase display. AJNR Am I Neuroradiol 1997;18:351-8.

13. Mascalchi $M$, Cosottini $M$, Ferrito $G$, et al. Contrastenhanced time-resolved $M R$ angiography of spinal vascular malformations. I Comput Assist Tonogr 1999;23:341-5.

14. Mascalchi $M$, Ferrito $G$, Quilici $N$, et al. Spinal vascular malformations: $M R$ angiography after treatment. Radiology 2001;219:346-53.

15. Pattany PM, Saraf-Lavi E, Bowen BC. MR angiography of the spine and spinal cord. Top Magn Reson Imaging 2003;14:444-60.

16. Saraf-Lavi E, Bowen BC, Quencer RM, et al. Detection of spinal dural arteriovenous fistulae with $M R$ imaging and contrast-enhanced MR angiography: sensitivity, specificity, and prediction of vertebral level. AJNR Am J Neuroradiol 2002;23:858-67.

17. Shigematsu $Y$, Korogi $Y$, Yoshizumi $K$, et al. Three cases of spinal dural AVF: evaluation with first-pass, gadolinium-enhanced, three-dimensional MR angiography. J Magn Reson Imaging 2000;12:949-52.

18. Hyodoh $\mathrm{H}$, Kawaharada $\mathrm{N}$, Akiba $\mathrm{H}$, et al. Usefulness of preoperative detection of artery of Adamkiewicz with dynamic contrast-enhanced MR angiography. Radiology 2005;236:1004-9.

19. Svensson LG. Intraoperative identification of spinal cord blood supply during repairs of descending aorta and thoracoabdominal aorta. I Thorac Cardiovase Surg 1996;112:1455-60; discussion 1460-1.

20. Williams GM, Perler BA, Burdick JF, et al. Angiographic localization of spinal cord blood supply 
and its relationship to postoperative paraplegia. I Vasc Surg 1991;13:23-33; discussion 33-5.

21. Thron A. Anatomy of the spinal cord's blood supply. In: Thron A, ed. Vascular anatomy of the spinal cord. Wien: Springer-Verlag; 1988:8-12 .

22. Thron A. Vascular anatomy of the spine. In: Byrne JV, ed. Interventional Neuroradiology. 1st ed. Oxford: Oxford University Press; 2002:19-23.

23. Zarr JH. Biostatistical analysis. London: Prentice Hall International Editions; 1996:163-78.

24. Bowen BC, Fraser K, Kochan JP, et al. Spinal dural arteriovenous fistulas: evaluation with $M R$ angiography. AJNR Am I Neuroradiol 1995;16:2029-43.

25. Binkert CA, Kollias SS, Valavanis A. Spinal cord vascular disease; characterization with fast threedimensional contrast-enhanced MR angiography. AINR Am J Neuroradiol 1999;20:1785-93.

26. Willinek WA, Gieseke J, Conrad R, et al. Randomly segmented central k-space ordering in high-spatialresolution contrast-enhanced MR angiography of the supraaortic arteries: initial experience. Radiology 2002;225:583-8.

27. Willinsky $R$, Lasjaunias $P$, Terbrugge $K$, et al. Angiography in the investigation of spinal dural arteriovenous fistula. A protocol with application of the venous phase. Ne'uroradiology 1990;32:114-6.

28. Luetmer PH, Lane JI, Gilbertson JR, et al. Preangiographic evaluation of spinal dural arteriovenous fistulas with elliptic centric contrastenhanced MR angiography and effect on radiation dose and volume of iodinated contrast material. AJNR Am J Nellorndiol 2005;26:711-8.

29. Guzman R, Dubach-Schwizer S, Heini P, et al. Preoperative transarterial embolization of vertebral metastases. Eur Spine J 2005;14:263-8.

30. Berenstein A, Lasjaunias P. Spine and spinal cord vascular lesions. In: Surgical Netroangiography, Vol 5, 1st ed. Berlin: Springer-Verlag; 1992:1-85. 


\section{Chapter VI}

MR angiography of the Adamkiewicz artery and anterior radiculomedullary vein: postmortem validation

Ry Nijenhuis MJ Jacobs MA van Engelshoven WH Backes 


\section{INTRODUCTION}

$M R$ angiography has recently emerged as an alternative imaging technique to intraarterial catheter (X-ray) angiography, also known as digital subtraction angiography, for depiction of the spinal cord arteries, in particular the Adamkiewicz artery (AKA)1-8, however, because of the similar spatial course and close vascular anatomy of the spinal cord arteries and veins, separation of these vessels is rather difficult. To the best of our knowledge, no validation of the use of MR angiography for imaging the spinal cord arteries and veins has been provided. This case report describes a postmortem validation of the localization of the AKA and the anterior radiculomedullary vein by using preoperative MR angiography in a patient who underwent open thoracoabdominal aortic aneurysm (TAAA) repair.

\section{CASE REPORT}

A 72-year old woman was admitted to our hospital and scheduled for open surgical repair of a Crawford type III TAAA. Preoperative MR angiography was performed to identify the segmental artery from which the AKA derived. Two consecutive dynamic phase MR angiography scans (Figure 6.1), 40 seconds each, were acquired by using a threedimensional spoiled gradient echo sequence with centrally ordered k-space filling, the start of which was synchronized with the arrival of $0.3 \mathrm{mmol} / \mathrm{kg}$ dose gadolinium contrast agent in the lower aorta ${ }^{1}$. Voxel sizes were $0.8 \times 0.8 \times$ $1.2 \mathrm{~mm}$ at acquisition. Both phases were transferred to an image-processing workstation for curved multiplanar reformation (MPR).
The AKA arose from the left 8th thoracic segmental artery (SA) according to the first phase angiogram. The anterior median vein drained to the epidural space of the left 12th thoracic segmental vein according to the second phase angiogram (Figure 6.1). The first phase angiogram selectively depicted the arteries (anterior cord surface). The second phase angiogram depicted the arteries (anterior cord surface) and veins (anterior and posterior cord surface; Figures 6.1 \& 6.2). A curved MPR of the posterior cord surface showed the posterior median vein and two posterior radiculomedullary veins (Figure 6.3). The largest radiculomedullary vein draining the thoracolumbar cord is called the great radiculomedullary vein, and in this case it was located on the posterior cord surface (Figure 6.3). The following day the patient underwent surgery. Reconstruction of the aorta was performed from the 9th thoracic segmental artery down to the aortic bifurcation with reattachment of the renal and visceral arteries. No postoperative neurologic complications occurred. Unfortunately, the patient died of pulmonary complications two weeks after surgery. At autopsy, the spinal cord was exposed to unravel the course and origin of the AKA and the anterior radiculomedullary vein. The AKA was found at the left T8 vertebral level and the anterior radiculomedullary vein at the left T12 vertebral level both in agreement with the preoperative $\mathrm{MR}$ angiography (Figure 6.1).

\section{DISCUSSION}

The major challenge in spinal MR angiography is to selectively depict the very small arteries of the spinal cord. This is difficult to 


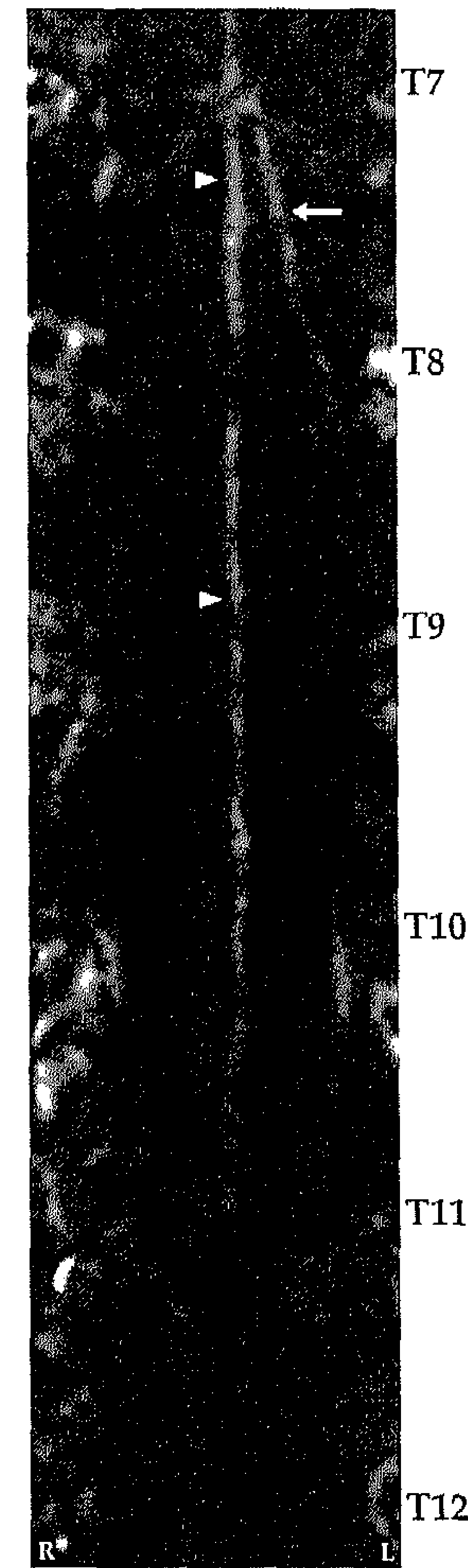

a

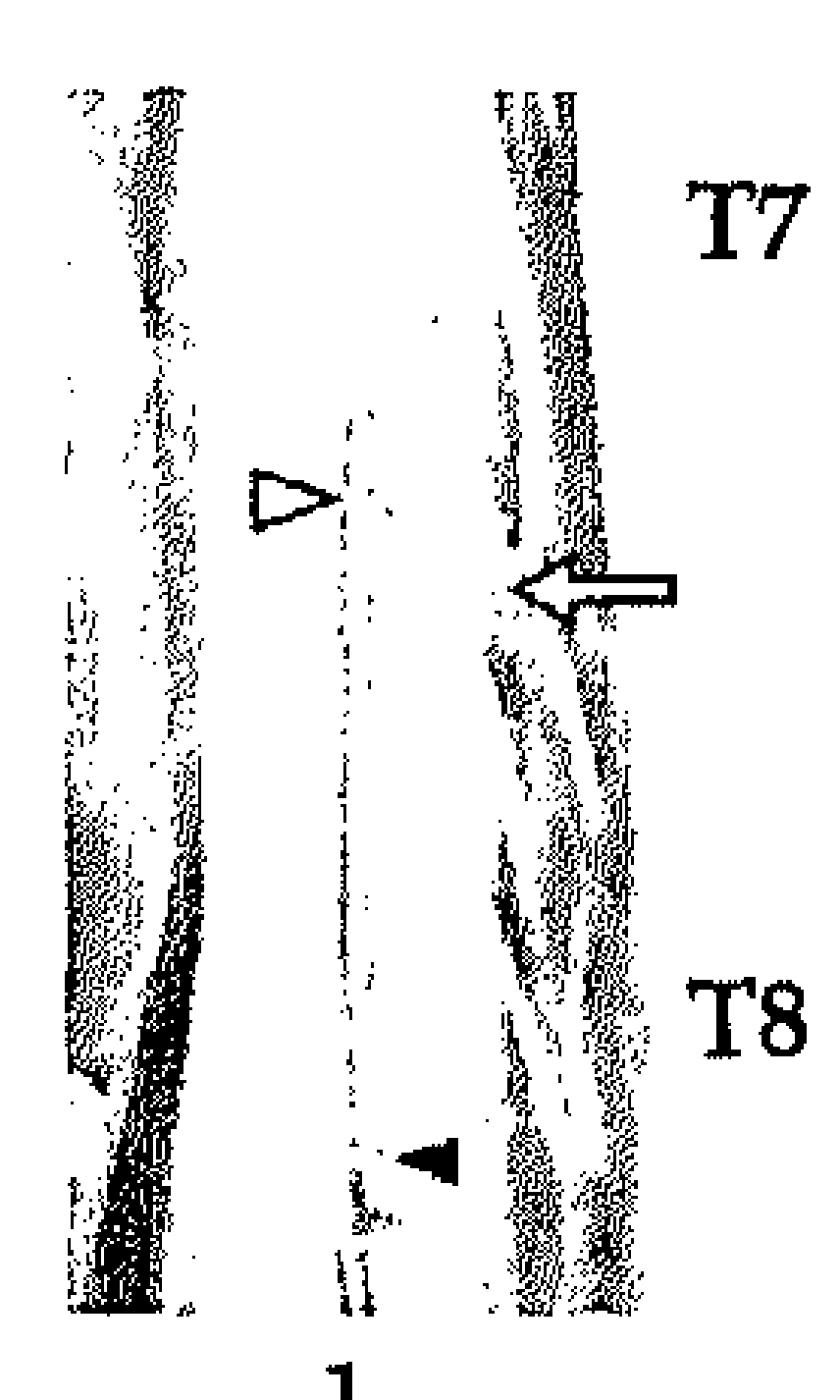

b

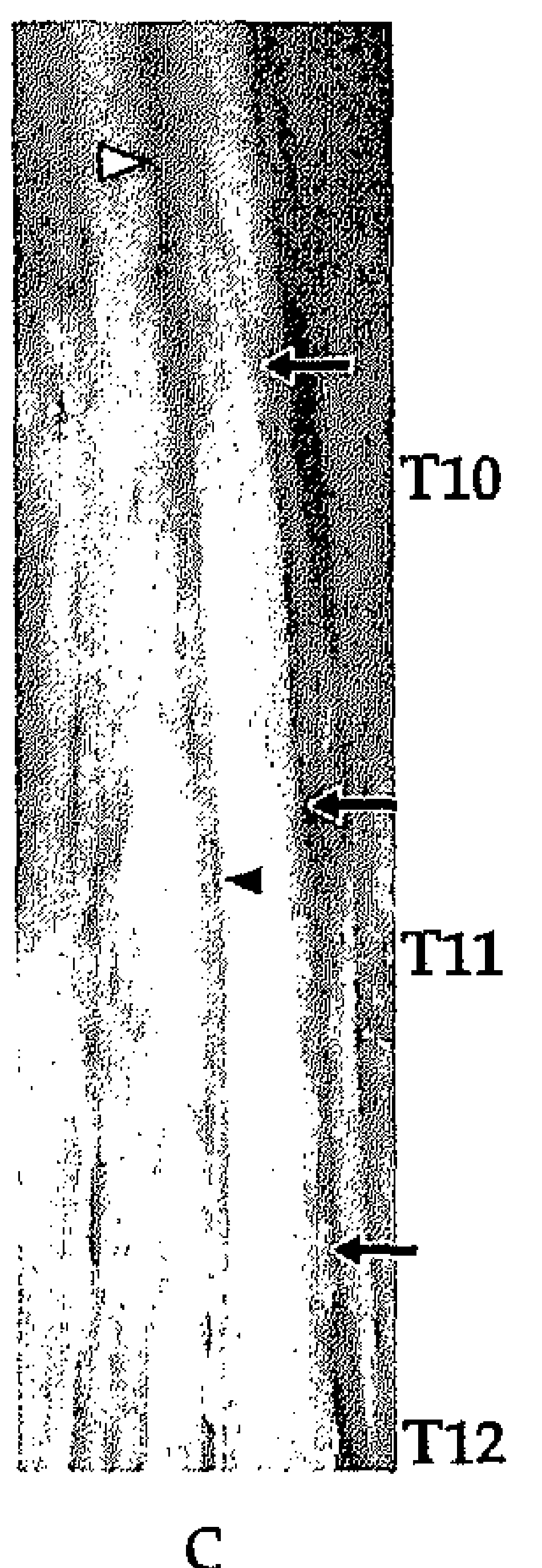

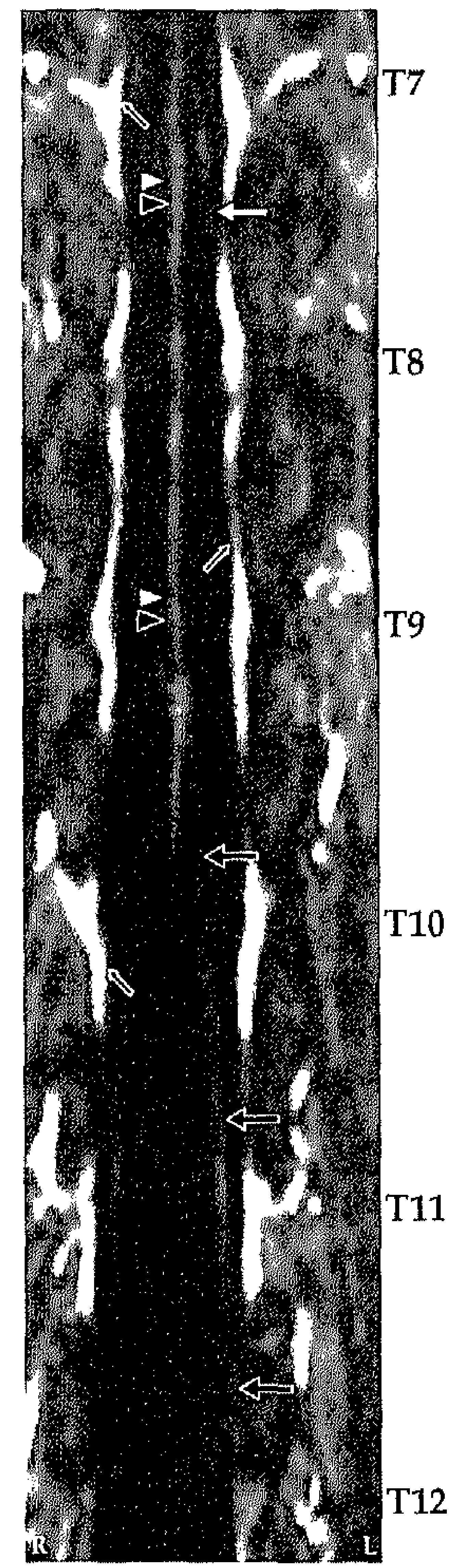

d

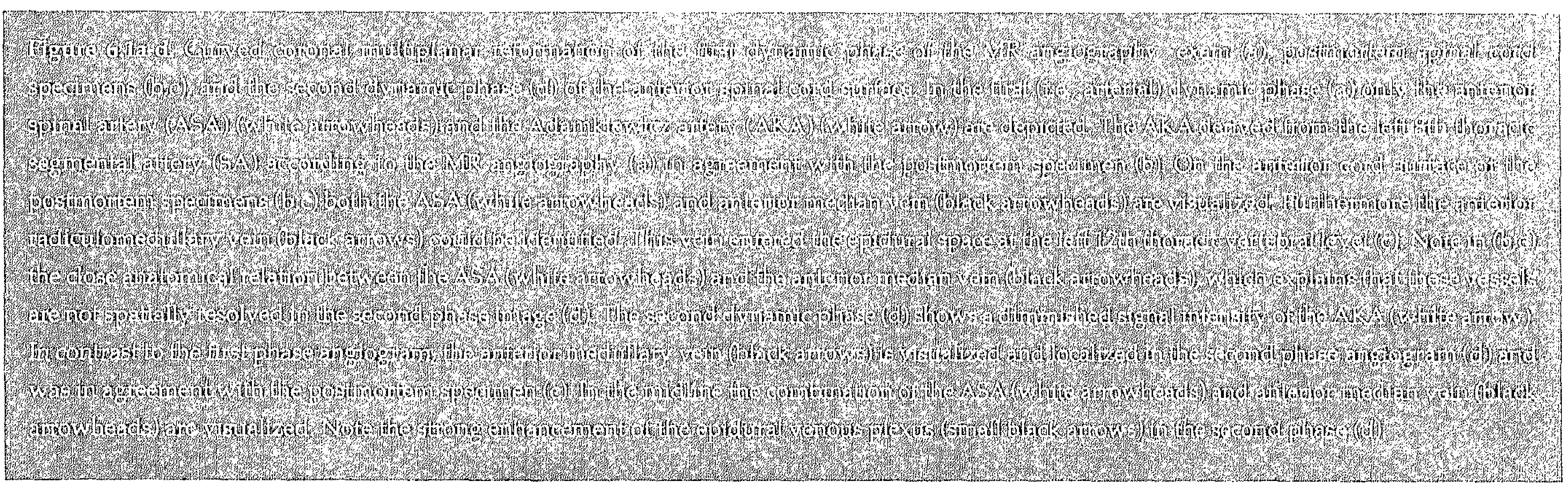


Postmortem validation

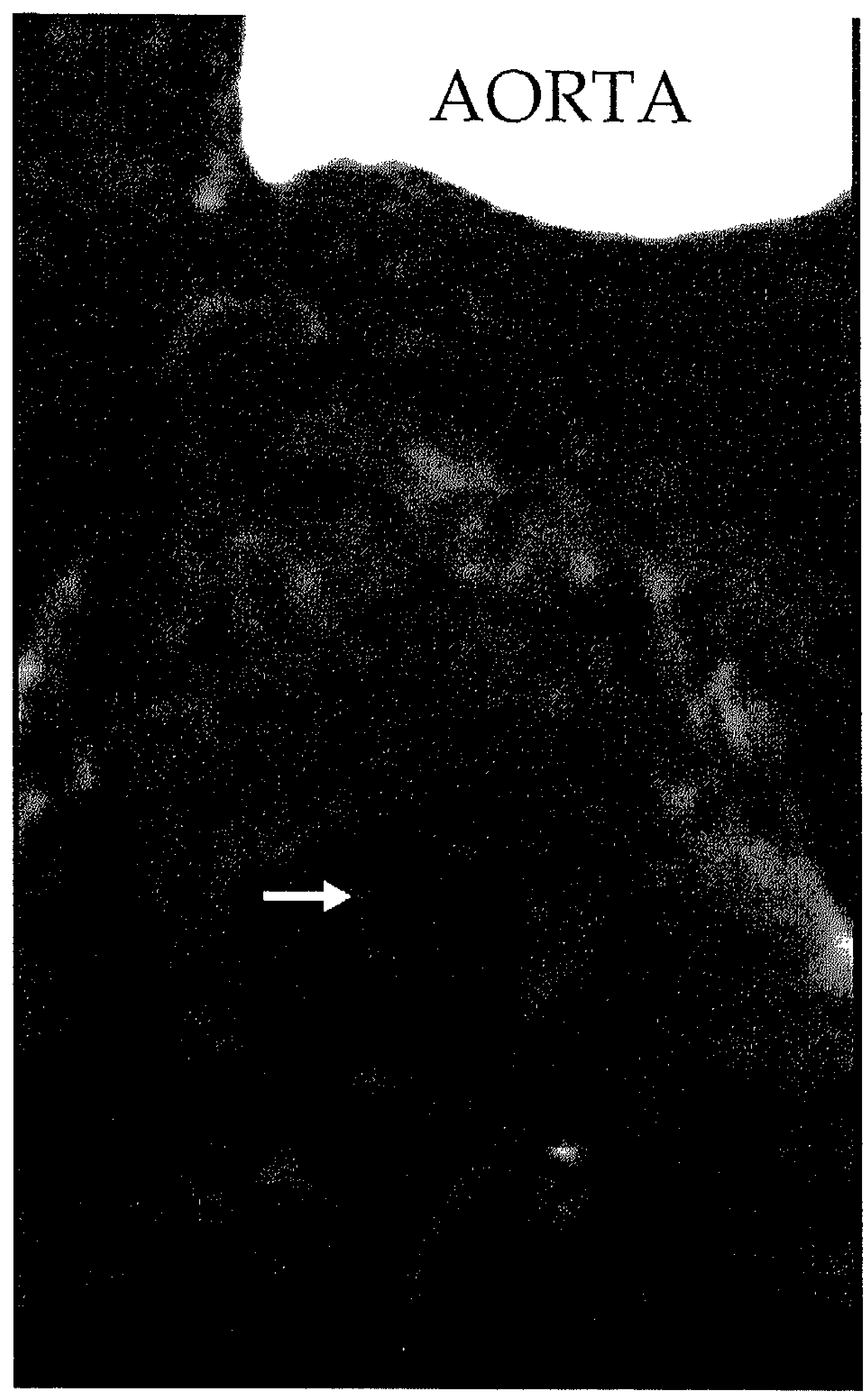

a

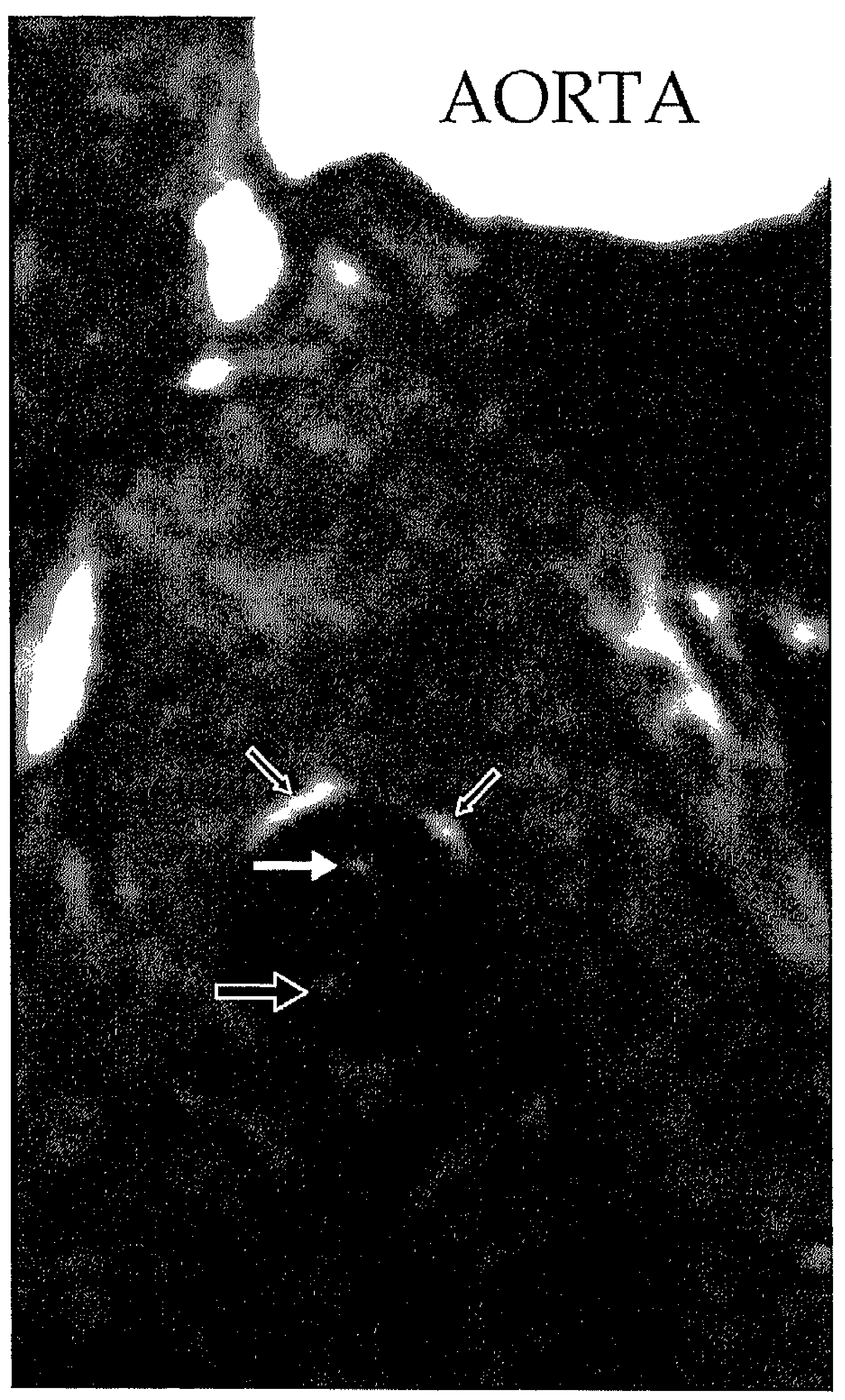

b

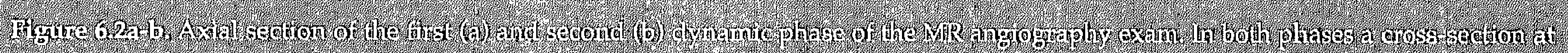

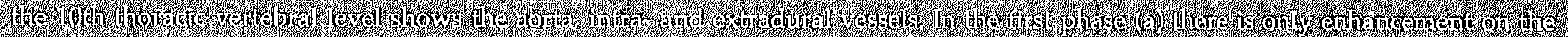

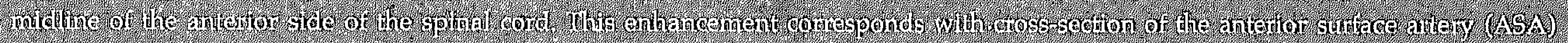

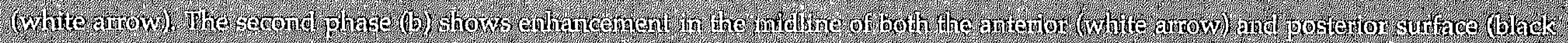

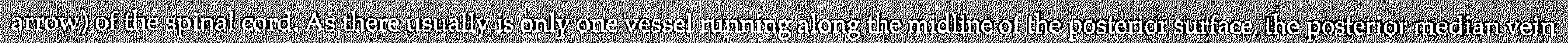

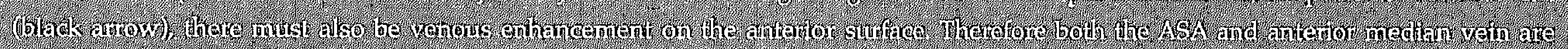

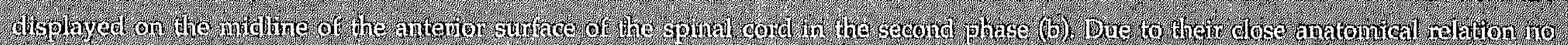

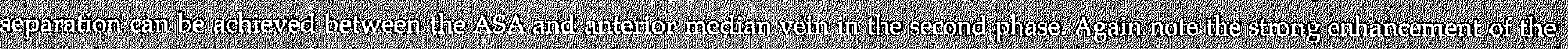

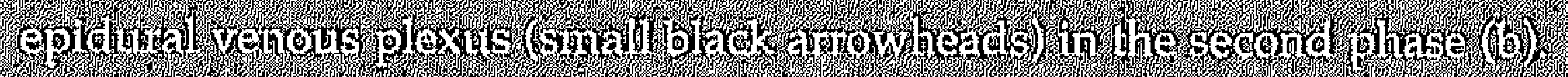



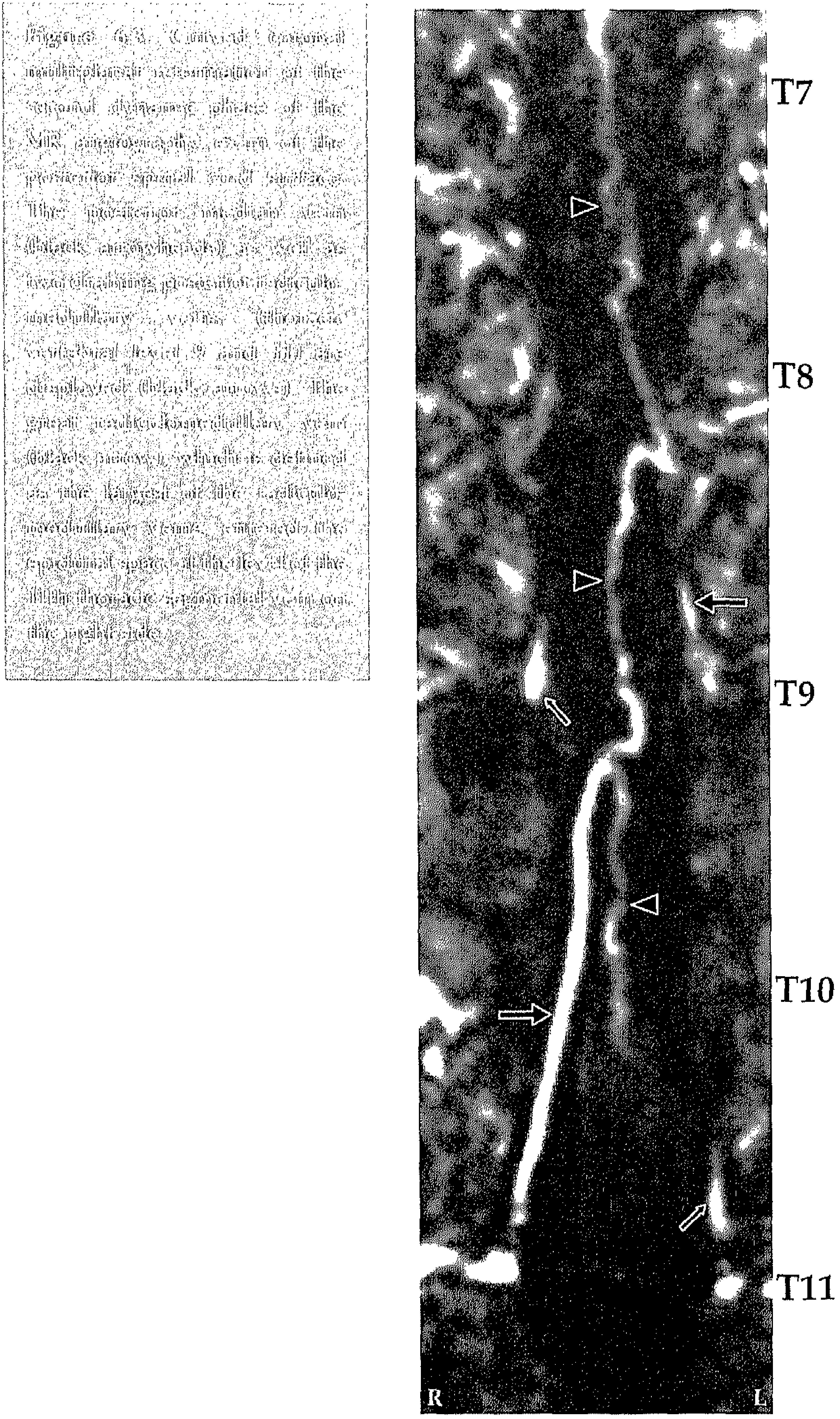

realize because (i) spinal cord arteries and veins are spatially close and have very small calibers that may only (partly) fill in one imaging voxel, (ii) spinal cord veins are usually thicker but have a similar configuration that easily may lead to misinterpretation between arteries and veins, and (iii) the AKA may originate from a strongly variable craniocaudal location (T5L2). So far, several MR angiography techniques have been attempted. Blood flowdependent techniques such as $3 \mathrm{D}$ phase contrast angiography ${ }^{9-11}$ and 3D CE time-offlight imaging 12,13 were the first techniques to be used. These flow-dependent techniques are unfortunately not able to fulfill all 3 requirements. Two-phase $\mathrm{MR}$ imaging featuring rapid contrast medium injection with a high-speed sophisticated acquisition sequence seems to be more capable of simultaneously providing the necessary features ${ }^{1-8,14-17}$. The spinal MR angiography protocol used here offers sufficient spatial resolution and effective temporal resolution and covers a large field of view, to overcome these conflicting requirements, respectively. Nevertheless, validation of this technique is necessary. The obvious validation technique is catheter angiography. Catheter angiography catheterization can be performed only by specialists and is not without risks in TAAA patients ${ }^{18}$, has a variable and limited sensitivity 18,19 , and is hampered by the occlusion of orifices of possibly many segmental arteries, which explains why spinal cord catheter angiography is not performed in the work-up of our TAAA patients. MR angiography is an attractive alternative imaging technique to localize the important AKA before TAAA surgery ${ }^{2-8}$. 
The current postmortem specimen provided the opportunity to validate the location of the AKA and the anterior radiculomedullary vein as obtained by preoperative MR angiography. This case demonstrated that MR angiography is an imaging technique that is able to depict the AKA and anterior spinal artery (ASA) correctly and temporarily separate these arteries from the intradural veins in a TAAA patient. 
Postmortem validation

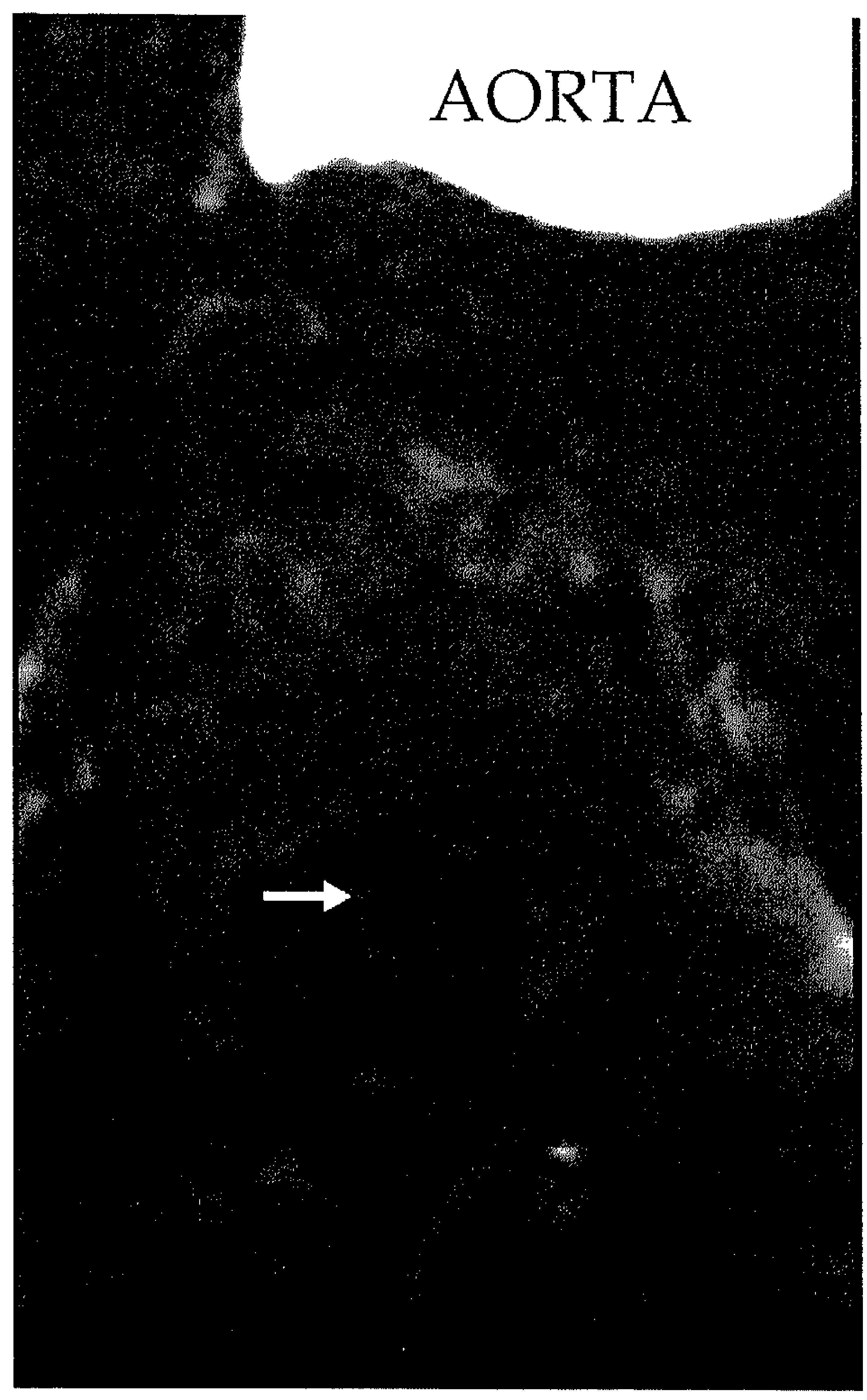

a

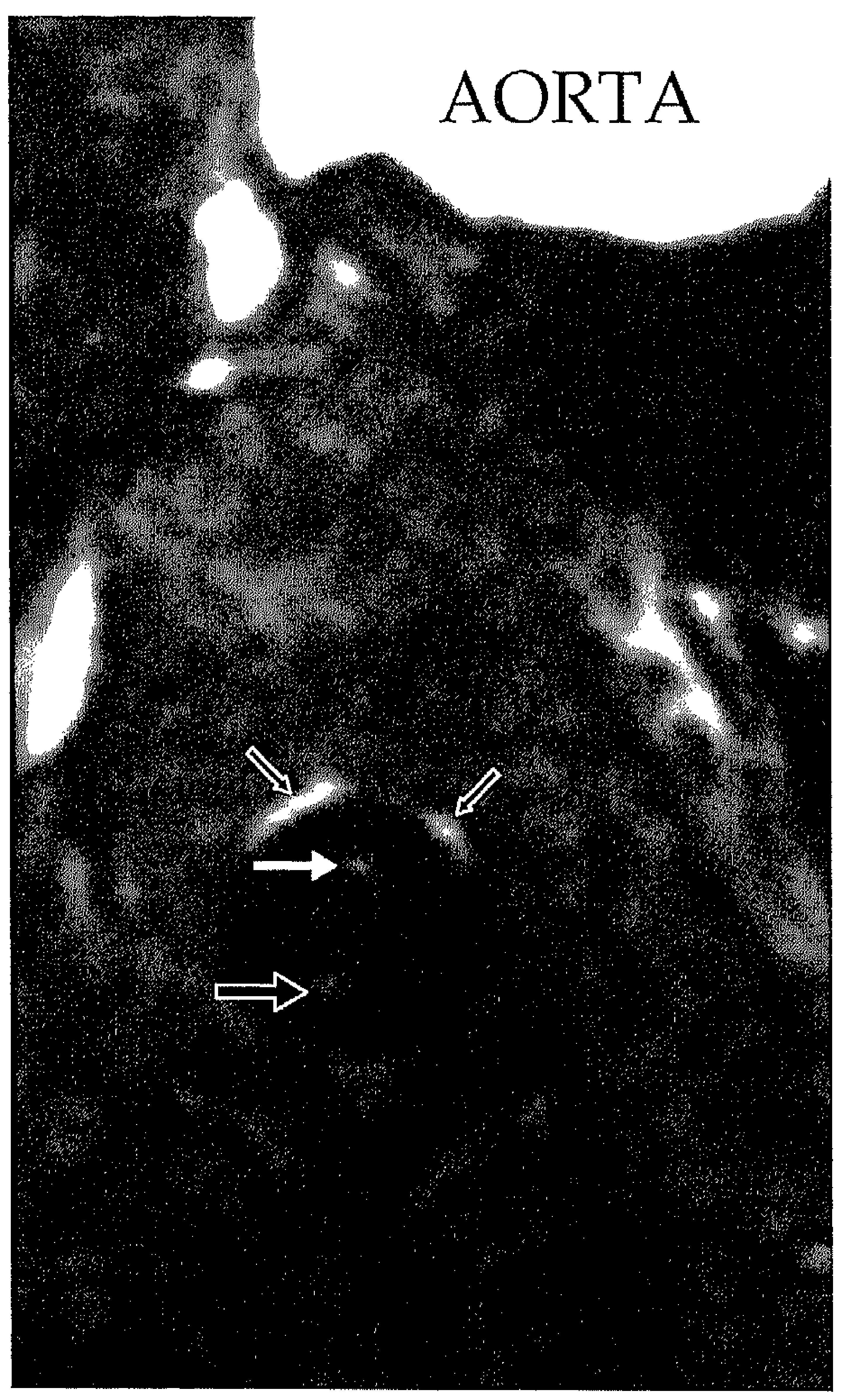

b

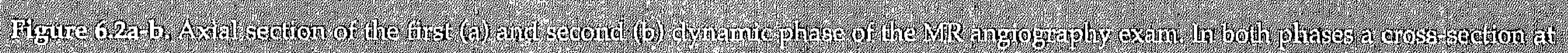

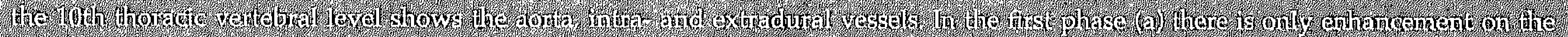

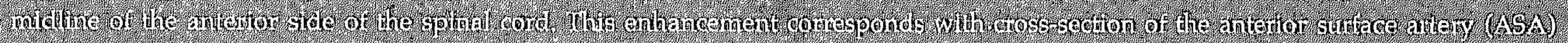

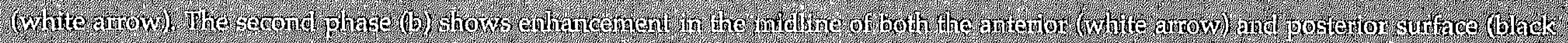

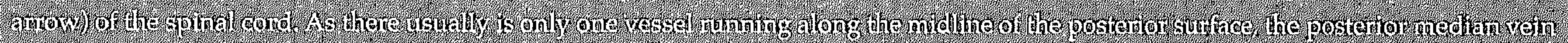

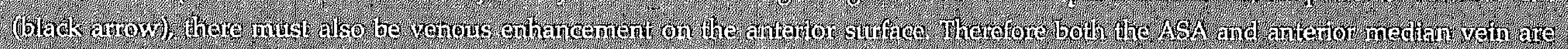

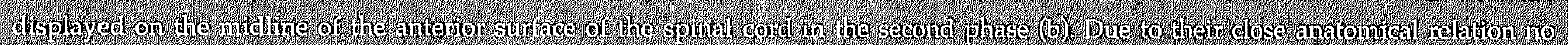

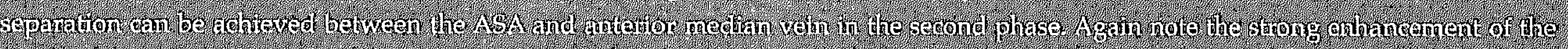

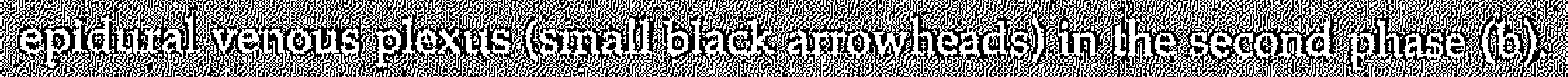




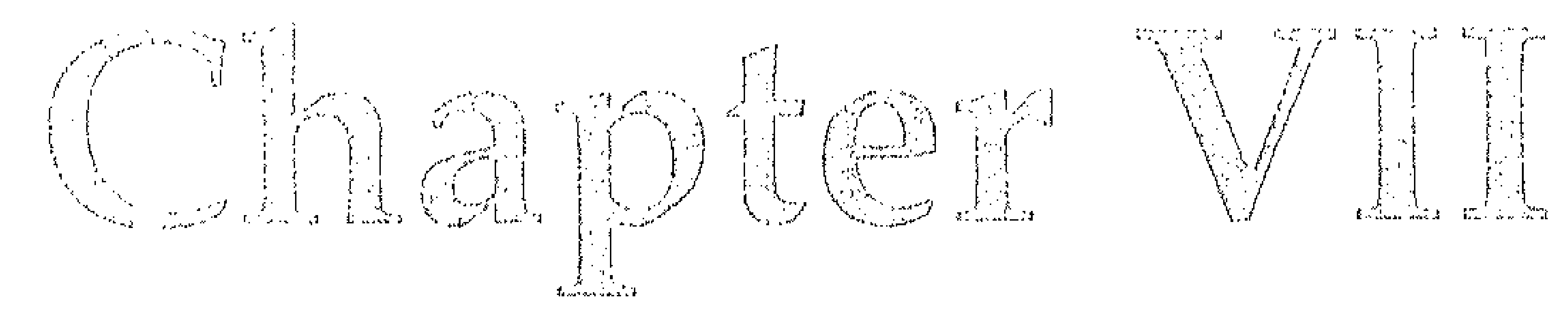

Comparison of MR with CT angiography for preoperative localization of the Adamkiewicz artery in thoracoabdominal aortic aneurysm patients

\author{
18) Bijonhuis \\ Nyl hacobs \\ KJ Jaspers \\ Theiner \\ Mreijnders \\ MA van Engedthoven \\ wh Hackes
}

foumal of Vascuhe Surgen 2007/45:677-45 


\section{ABSTRACT}

Purpose: Preoperative localization of the Adamkiewicz artery (AKA) and its segmental supplier in advance to thoracic (TAA) and thoracoabdominal aortic aneurysm (TAAA) repair is proposed to be useful to prevent postoperative paraplegia. The diagnostic potential of magnetic resonance (MR) and computed tomography (CT) angiography was evaluated for the preoperative localization of the AKA in Caucasian TAAA patients.

Materials and Methods: Thirty-nine consecutive patients with a TAA(A) scheduled for elective open surgical aortic repair preoperatively underwent $\mathrm{MR}$ and $\mathrm{CT}$ angiography. Objective image quality was assessed by measuring the signal-to-noise ratio (SNR) and contrast-to-noise ratio (CNR) of the AKA and was related to patient thickness. Two independent observers scored the location of the AKA and the subjective image quality of vessel to background contrast of the AKA, image noise, spinal cord tissue enhancement, epidural venous enhancement, and overall image quality.

Results: Average detection rate for AKA localization was $71 \%(67-74 \%)$ for CT angiography and 97\% (94-100\%) for MR angiography. Interobserver agreement was $82 \%$ for CT angiography and $94 \%$ for MR angiography. SNR was significantly higher $(P<0.001)$ and CNR was significantly $(P<0.001)$ lower for $C T$ angiography compared to $M R$ angiography. Contrast of the AKA $(P<0.001)$ and overall image quality $(P<0.004)$ were judged to be significantly better for MR angiography. Spinal cord tissue enhancement was judged stronger at $C T$ angiography $(P<0.03)$, with significantly less epidural venous enhancement $(P<0.001)$. No significant difference was found in image noise. SNR and CNR decreased significantly $(P<0.001)$ with increasing patient thickness for $C T$ angiography but not for MR angiography.

Conclusions: Localization of the AKA in Caucasian TAAA patients is possible with both CT and MR angiography. Compared to $\mathrm{CT}$ angiography, MR angiography is more favourable considering the higher AKA detection rate, the higher contrast-to-noise ratio and its independence of patient thickness. 


\section{INTRODUCTION}

Patients undergoing thoracic (TAA) or thoracoabdominal aortic aneurysm (TAAA) repair are at risk for developing ischemic spinal cord injuries such as paraparesis or paraplegia. To avoid these dreadful complications preoperative visualization of the spinal cord blood supply has been suggested and employed ${ }^{1-9}$. The largest, and therefore considered to be the most important supplier of the thoracolumbar spinal cord is the great anterior radiculomedullary artery (i.e., the Adamkiewicz artery $[\mathrm{AKA}])^{10}$. The AKA has a calibre of $<1.0 \mathrm{~mm}$ and derives from a posterior branch of an aortic segmental artery ${ }^{11}$. Since the AKA supplies the largest and most vulnerable part of the thoracolumbar spinal cord it is the artery of interest in preoperative diagnostic imaging.

Different imaging techniques can be used to depict the AKA. The standard of reference for this purpose is intra-arterial catheter (X-ray) angiography, also known as digital subtraction angiography. However, this technique is invasive and can be technically difficult to perform in TAAA patients, which is illustrated by a highly variable AKA detection rate (43$86 \%)^{1,2}$. Moreover, catheter angiography can be hazardous to the vascular patient as severe complications, including paraplegia, have been described ${ }^{1}$. Although the risk for major complications in the largest published series of 480 patients was only $1.2 \%$ it is significant compared to the occurrence of paraplegia due to surgery in our TAAA patient population (3$5 \%)^{12}$. Alternatively, non-invasive magnetic resonance (MR) and computed tomography (CT) angiography ${ }^{3-9,13-18}$ have been explored to investigate their potential to localize the AKA and its segmental supplier.
Recently, we have shown with two validation studies ${ }^{14,15}$ that MR angiography is able to consistently localize the AKA and its segmental supplier and differentiate it from the similarly shaped great anterior radiculomedullary vein (GARV). Furthermore, we have performed a study in 60 TAAA patients that revealed two important outcomes ${ }^{5}$. First of all, MR angiography can preoperatively localize the AKA in up to $100 \%$ of the TAAA patients. Secondly, there is a significant correlation between the location of the segmental artery supplying the AKA relative to the aortic cross-clamped area and intraoperative spinal cord function. It was found that when the segmental supplier of the AKA was outside the cross-clamped area no changes in spinal cord function were observed. This means that preoperative localization of the AKA by MR angiography has a negative predictive value of $100 \%$ for a decrease in spinal cord function. On the other hand, when the segmental supplier was located inside the cross-clamped area the positive predictive value was $32 \%$ for a decline in spinal cord function. The results indicated that the AKA indeed is still functional in TAAA patients and that maintenance of its direct segmental supply is crucial in one third of the patients with a temporary decrease in spinal cord function during surgery.

The results show that MR angiography can reliably detect the AKA. However, there is no report on the detection ratio of $\mathrm{CT}$ angiography in Caucasian TAAA patients. The use of $C T$ angiography instead of MR angiography would from a logistical point of view be beneficial, as CT angiography is generally used to determine the diameter, extent, and type (atherosclerotic or dissection) 
of the aortic aneurysm. To our knowledge there are only two studies, both performed in Japanese patients, in which CT and MR angiography were compared for the localization of the AKA in the same patients 9,16 .

The aim of this study was to investigate whether MR or CT angiography is the preferred technique for the preoperative visualization of the AKA in Caucasian TAA(A) patients. To this end, the AKA detection rate as well as the quantitative and observational image quality were compared between $\mathrm{CT}$ and MR angiography.

\section{MATERIALS and METHODS}

\section{Patients}

From September 2002 to May 2005, 39 consecutive patients (20 male and 19 female) with either a TAA or a TAAA scheduled to undergo elective surgical aortic aneurysm repair were included in this study. These patients were also included in a previous study which correlated the location of the segmental artery supplying the AKA relative to the aortic cross-clamped area with intraoperative spinal cord function ${ }^{5}$. The 39 patients (age $65 \pm 12$ years; mean \pm SD) could be divided in five different groups according to the extent of their aortic pathology using the Crawford classification: 16 TAA, 2 TAAA type I, 12 TAAA type II, 4 TAAA type III, and 5 TAAA type IV patients. Fourteen patients had chronic type-B dissection and to some extent atherosclerosis and 25 patients had advanced atherosclerotic disease. Prior to surgery all patients underwent CT and MR angiography. CT angiography preceded $\mathrm{MR}$ angiography in all patients. The interval between these two examinations varied from one day to three weeks. CT angiography was performed as part of the regular clinical workup program prior to surgery to determine the extent and location of the aortic pathology.

The medical ethics committee of the hospital approved the study protocol and written informed consent was obtained from all patients prior to inclusion.

\section{CT angiography}

CT angiography images were obtained on a 4-channel multi-detector row CT scanner (Aquilion; Toshiba, Tokyo, Japan). Via the antecubital vein $120-150 \mathrm{~mL}$ of iohexol (350 $\mathrm{mg} / \mathrm{mL}$ Iodine, Omnipaque, GE Health, Oslo, Norway) was injected at a rate of $3 \mathrm{~mL} / \mathrm{sec}$. The scanning range was set from the level of the apex of the lungs down to the lower edge of the sacrum. Initially, the contrast bolus arrival timing in the ascending aorta was assessed according to the protocol of Takase et $\mathrm{al}^{4}$. (Sure Start, Toshiba, Tokyo, Japan). However, employing this injection protocol to the first six patients did not provide satisfying depiction of spinal cord arteries (or veins). These patients were not included in this study and the contrast timing was adjusted. A fixed scan delay of 35 seconds was used in the 39 included patients before starting the contrastenhanced acquisition. The technical parameters for the first 12 patients were as follows: collimation width $4 \times 2 \mathrm{~mm}$, pitch 0.875 , reconstructed slice width $2.0 \mathrm{~mm}$, reconstruction increment $1.5 \mathrm{~mm}$, standard abdominal filter (FC01), X-ray tube rotation time 0.5 second, tube voltage $120 \mathrm{kV}$, and tube load $200 \mathrm{mAs}$. For the remaining 27 patients the parameters were adjusted to increase contrast. Adjusted technical parameters in the last 27 patients were rotation speed 1.0 second, $80 \mathrm{kV}$, and $300 \mathrm{mAs}$. For all patients only one 
dynamic phase was acquired with CT angiography to limit radiation exposure. Duration of the CT angiography examination was less than 15 minutes.

\section{$M R$ angiography}

All patients underwent preoperative MR angiography to localize the AKA and its segmental supplier. Imaging was performed on a clinical 1.5-Tesla MRI system (Philips Intera, Philips Medical Systems, Best, The Netherlands) equipped with a phased-array synergy surface spine coil. The MRI protocol included a bolus timing scan series, in which the time of contrast arrival in the distal aorta was determined. Subsequently, the MR angiography exam (consisting of two consecutive dynamic phases) was performed using a three-dimensional spoiled gradient echo sequence. A centric $\mathrm{k}$-space filling scheme synchronized to the arrival of the contrast agent (dose $45 \mathrm{~mL}$, gadopentetate dimeglumine, Magnevist, Schering, Berlin, Germany) was used to suppress venous blood signal. The craniocaudal field of view was 50 $\mathrm{cm}$, slice thickness was $1.2 \mathrm{~mm}$, matrix dimensions were $464 \times 512$, and the number of slices (76-110) was individually adjusted. The two dynamic phases (each 36-52 seconds) allowed differentiation based on temporal intensity changes between the AKA and the great anterior radiculomedullary vein (GARV), which has a similar spatial configuration. Details of the imaging protocol have been described elsewhere6,14,15. The MR angiography examination typically took 30 minutes to perform.

\section{Image analysis}

AKA localization. Image data sets were transferred to an image postprocessing workstation (Easy Vision, release 4.0; Philips Medical Systems, Best, the Netherlands). Image data sets were anonymized by a technical assistant not involved in the analysis. Each individual examination was assigned with a unique identification number resulting in $39 \mathrm{MR}$ and $39 \mathrm{CT}$ threedimensional image data sets. The 78 examinations were randomly presented to two experienced independent observers, blinded for the clinical details and each others results. These observers attempted to localize and visualize the trajectory from the aorta via the segmental artery and AKA to the spinal cord in each dataset using targeted maximum intensity projections and curved multiplanar reformations. In case this was not successful analysis of signal intensity time courses of the depicted vessels had to be performed. When the signal intensity of the depicted vessel decreased in the second phase this vessel was considered to be the AKA. If a vessel had the same signal intensity or showed an increase it was considered to be the GARV. This analysis of signal intensity time courses was only possible with MR angiography examinations. In cases where visualization of the arterial trajectory was not successful with CT angiography, differentiation of the AKA from the GARV was based on differences in morphologic features and craniocaudal localization. Frequently, the GARV has a longer intradural trajectory, is located more caudally, and/or has a larger caliber than the AKA. The segmental origin of the AKA and GARV at MR angiography was determined using the T2-weighted anatomic images in combination with the MR coil markers and late phase $\mathrm{MR}$ angiography images, which showed the contrast-enhanced vertebral bodies. 
For both CT and MR angiography the level could be determined by counting the vertebral bodies. The intervertebral foramen at which the spinal branch of the seqmental artery (SA) entered the spinal canal and continued as the AKA was interpreted as the AKA level. For instance, in case the spinal branch entered between the twelfth thoracic $(\mathrm{T})$ vertebra and the first lumbar $(\mathrm{L})$ vertebra, the localization was denoted as segmental level T12.

After each observer independently tried to localize the AKA, the percentage of agreement between the two observers was calculated for $\mathrm{CT}$ and MR angiography. Observations were considered in agreement in case both observers localized the AKA at the same level on the same side or when both observers did not detect the AKA.

Observational image quality. The same two observers who tried to localize the AKA were asked to give a subjective score on a 5 -point scale ranging from 0 (zero/none); 1 (very poor/weak); 2 (poor/weak); 3 (moderate); 4 (good/significant) to 5 (excellent/strong) regarding the following image quality items: spinal cord tissue enhancement, epidural venous enhancement, image noise, contrast of the AKA relative to the adjacent image region, and overall image quality for both image modalities and every patient. These scores were averaged per patient per image quality item.

Quantitative image quality. To assess objective image quality for both imaging modalities signal intensity (SI) measurements were performed by an independent (i.e., third) reader to determine the signal-to-noise (SNR) and contrast-to-noise ratio (CNR). Because a spine phased-array surface coil was used, no representative air regions were available to obtain reliable noise measurements for MRI.
Therefore the standard deviation of the SI in the erector spinae muscle $\left(\mathrm{SD}_{0}\right)$ was determined to represent the noise level. SNR was calculated as the ratio between the SI of the artery and $\mathrm{SD}_{0}$. CNR was calculated as the difference between SI of the artery and the surrounding image region divided by $\mathrm{SD}_{0}$. For $\mathrm{CT}$ angiography the SI was measured in terms of Hounsfield units and converted to attenuation coefficients in $\mathrm{cm}^{2} / \mathrm{g}$.

To investigate the influence of the patients' body habitus on the SNR and CNR of the AKA the (transverse) anterior-posterior diameter of the abdomen at the level of T12 was measured. To assess the possible relation of SNR and CNR as a function of this diameter regression analysis was applied, to quantify the effect of the exponential decay of the radiation transmission with patient thickness.

\section{Surgical protocol}

The surgeon was informed about the localization of the segmental AKA supplier and other open and occluded segmental arteries. The operation strategy regarding the placement of the aortic cross-clamps was not influenced by the preoperative angiography results. The surgical procedure was performed in a similar manner as described before ${ }^{12,19,20}$. Our spinal cord protection protocol consisted of cerebrospinal fluid (CSF) drainage, left atrium-femoral artery or femoral arteryfemoral vein bypass enabling distal aortic perfusion and spontaneous cooling to $32-$ $33^{\circ} \mathrm{Celsius}$ with active rewarming at the end of the procedure.

Neuromonitoring of spinal cord function was performed intraoperatively by means of motor-evoked potentials (MEPs) and has been described in detail elsewhere ${ }^{12,19,20}$. In general, the aorta is sequentially cross- 


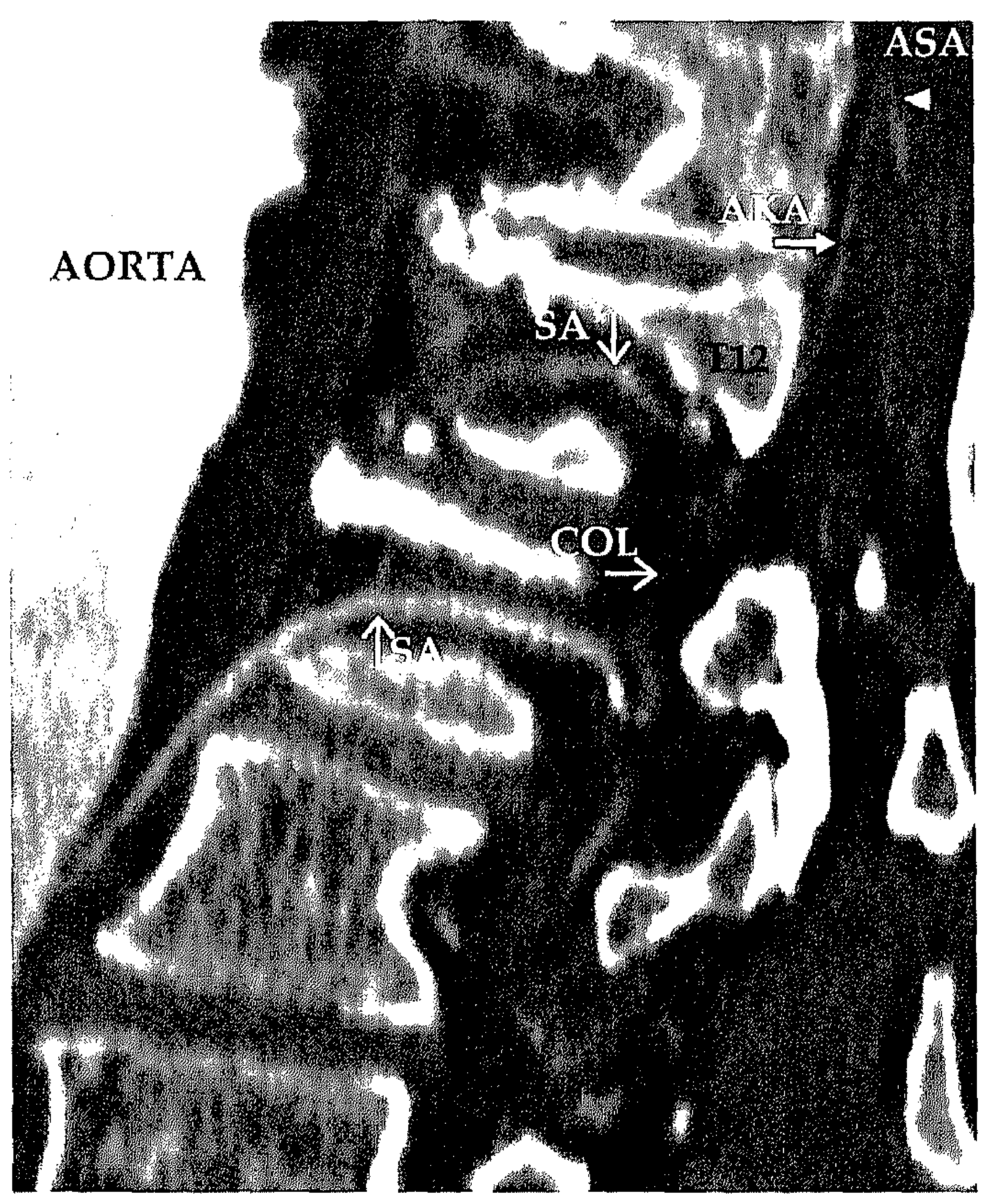

a

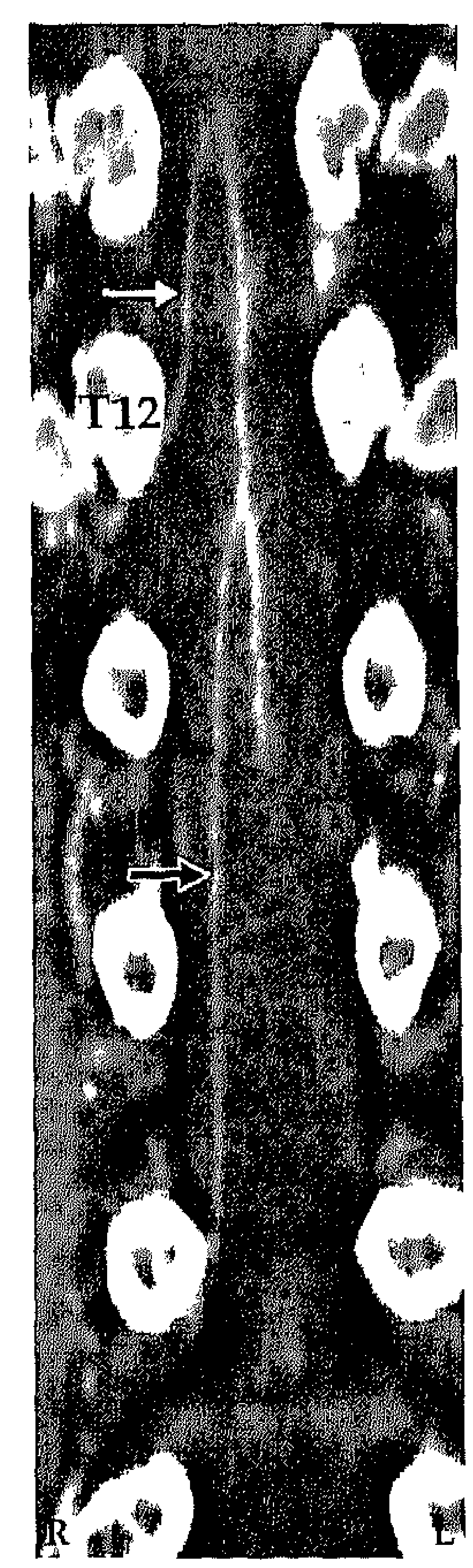

b

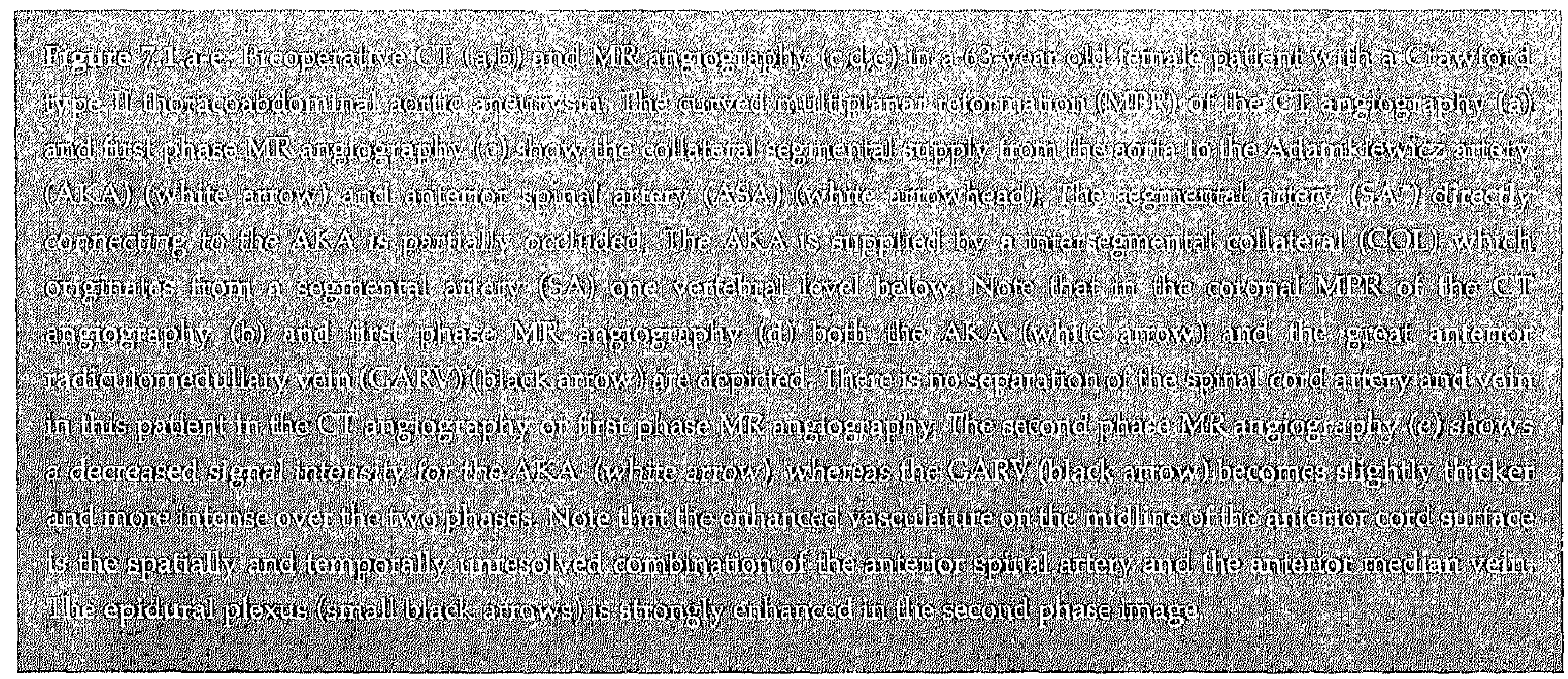


MR and CT angiography comparison

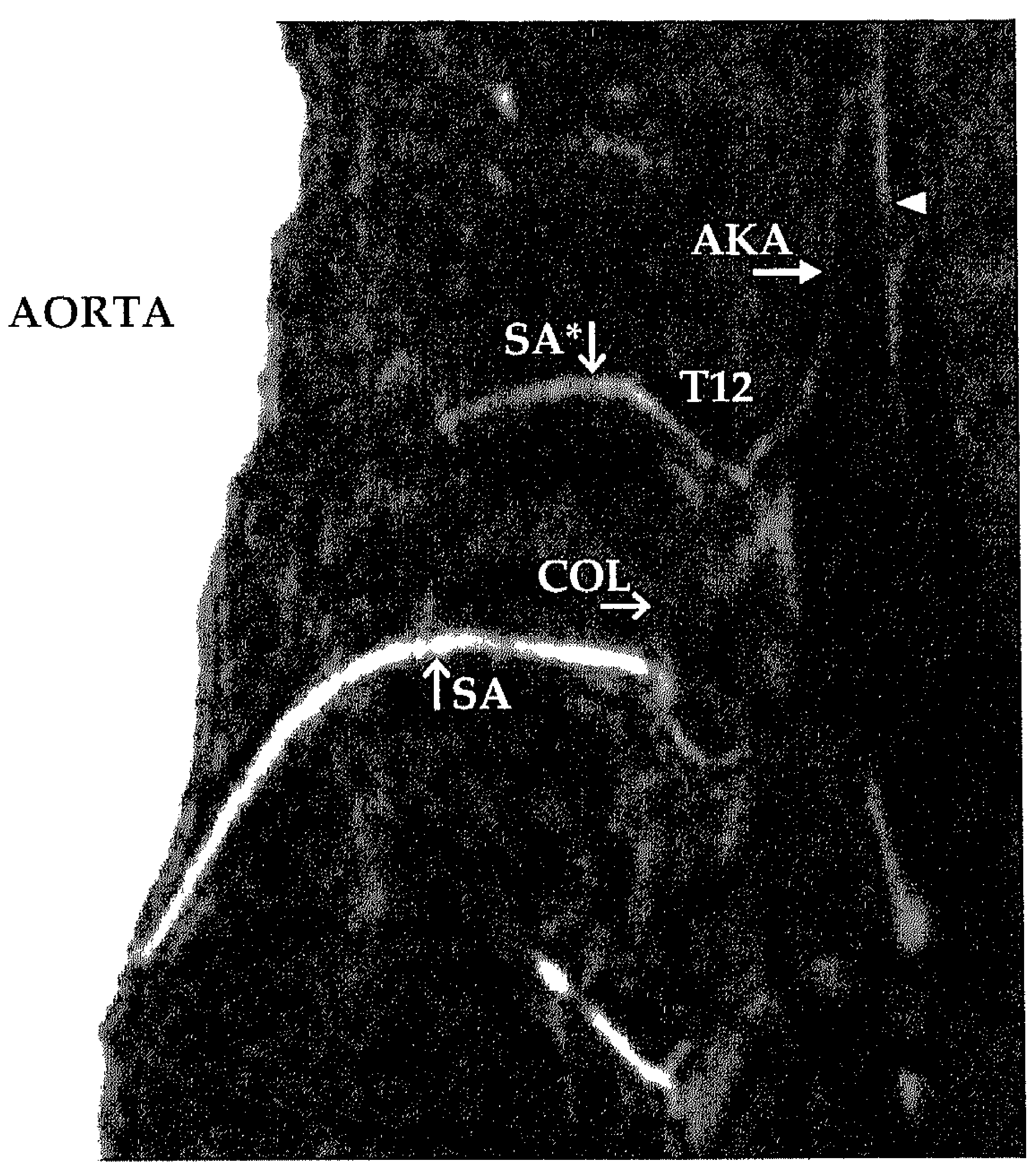

C

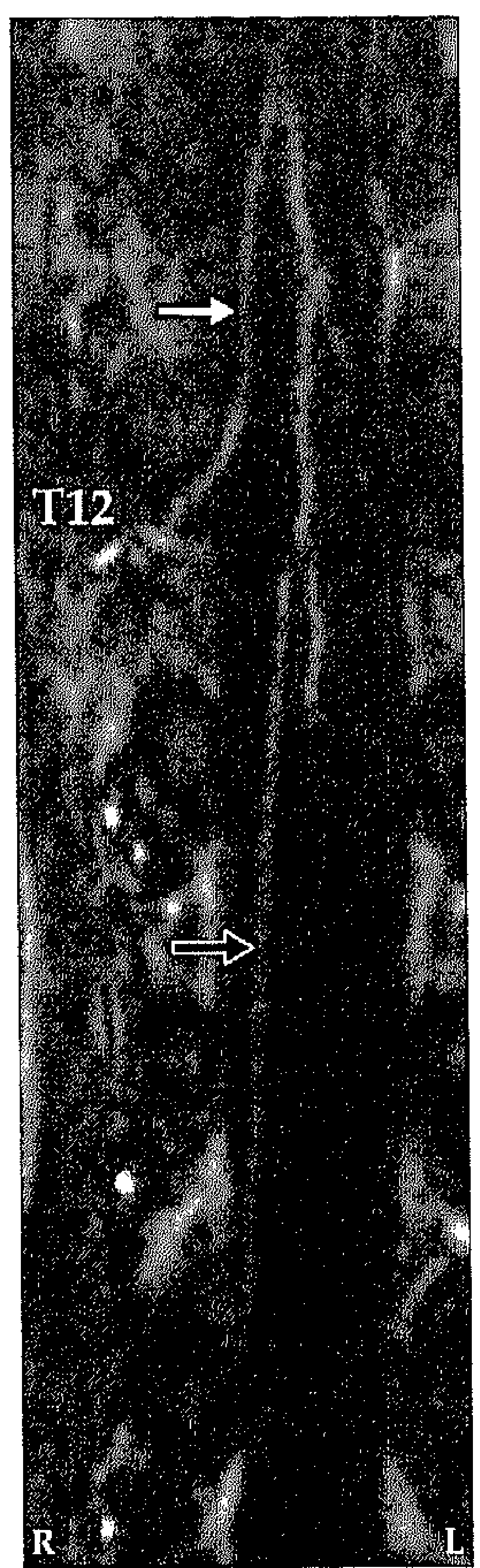

d

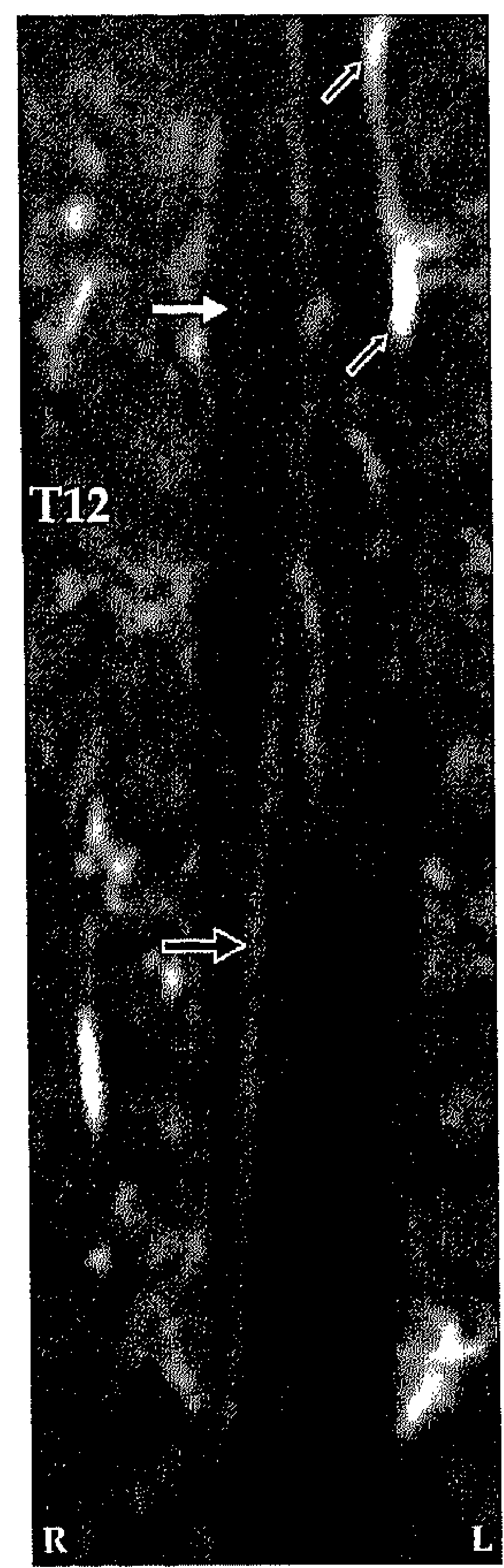

$\mathrm{e}$ clamped, allowing stepwise exclusion of aortic segments and assessment of changes in MEP amplitudes. If after placement of the proximal clamp the MEP decreased, the mean distal aortic perfusion pressure was increased till the MEP normalized. However, if during aortic cross-clamping the MEP rapidly decreased and were not correctable with increasing distal and mean arterial pressure, indicating that the excluded aortic segment contained crucial segmental arteries, the clamps were released and the patient was actively cooled to $32^{\circ} \mathrm{C}$, affording additional neuroprotection. The aorta was then clamped again and opened. Patent segmental arteries were reattached and perfused till the MEPs returned to baseline levels. When the origin of the SA supplying the AKA was inside the cross-clamped area this SA was reattached and perfused first. In case MEP amplitudes remained stable, several patent SAs were preventively reattached except when they arose from a severely diseased aortic wall. When possible revascularization of the hypogastric arteries was additionally performed. 
Table 7.1. Overview of the Adamkiewicz artery detection by both observers for CT (a) and MR angiography (b).

a

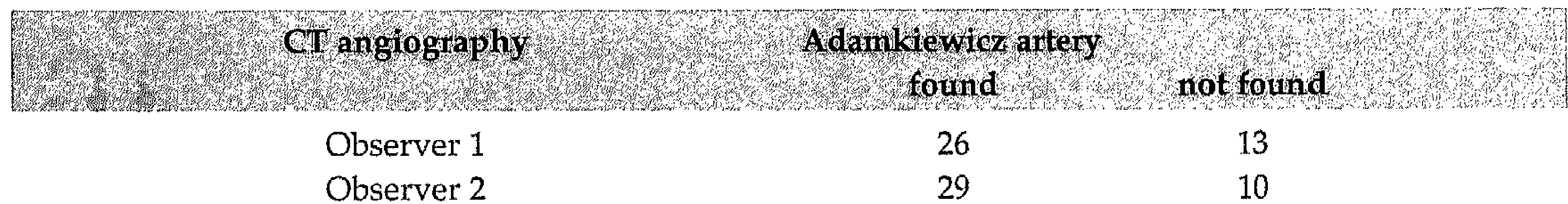

b

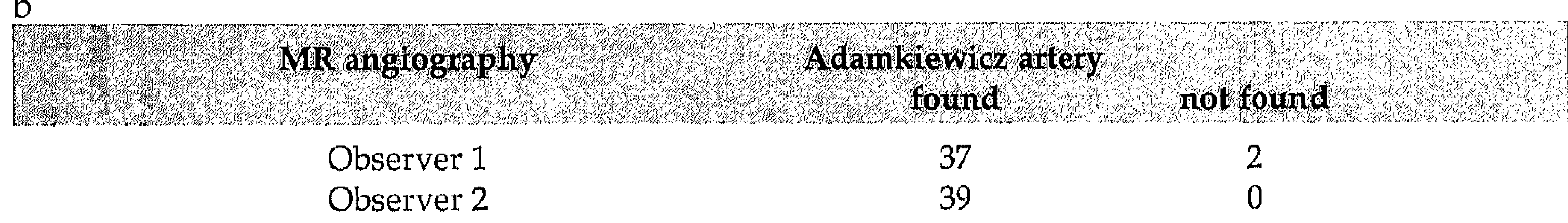

\section{Statistical analysis}

For statistical analysis SPSS software was used (version 11.0; SPSS Inc, Chicago, IL, USA). The paired-sample Student's t-test was used to test whether there were significant differences in SNR and CNR between the two imaging modalities. To test if there were significant differences in subjective image quality and AKA detection rates the Wilcoxon signed rank test was applied. Statistical significance was inferred when the obtained $P$-value was less than 0.05. Exponential-linear regression analysis was applied to investigate the possible relation between the SNR and CNR and patient thickness for both modalities.

\section{RESULTS}

\section{Image analysis}

AKA localization. In all 39 patients image data sets suitable for analysis were obtained with both modalities. No examination had to be aborted due to claustrophobia and no side effects occurred during or after the injection of contrast material. The average scan delay for
MR angiography was 27 seconds (range 16-55 seconds).

The AKA was more often detected by both observers with MR angiography compared to CT angiography (Table 7.1). The average detection rate with MR angiography was $97 \%$ (observer 1: 95\%; observer 2: 100\%), which was significantly $(P<0.001)$ higher compared to the average detection rate of $71 \%$ (observer 1: $67 \%$; observer 2: $74 \%$ ) for CT angiography (Figures 7.1 and 7.2). The AKA was localized in all patients between vertebral levels $\mathrm{T} 8$ and L1 and derived from the left side in $64 \%$ $(25 / 39)$ and in $36 \%(14 / 39)$ of the cases from the right side (Table 7.2). In all patients the AKA originated between vertebrae T8 and L1.

The GARV was observed in 19 patients with CT angiography and always covisualized with the AKA. Differentiation with CT angiography between AKA and GARV was based on vascular anatomy. With MR angiography the GARV was observed in 36 patients (Table 7.2). Here the GARV was observed 34 times in the early phase images and 36 times in the late phase images. Differentiation between AKA and GARV with MR angiography was possible on the basis of 


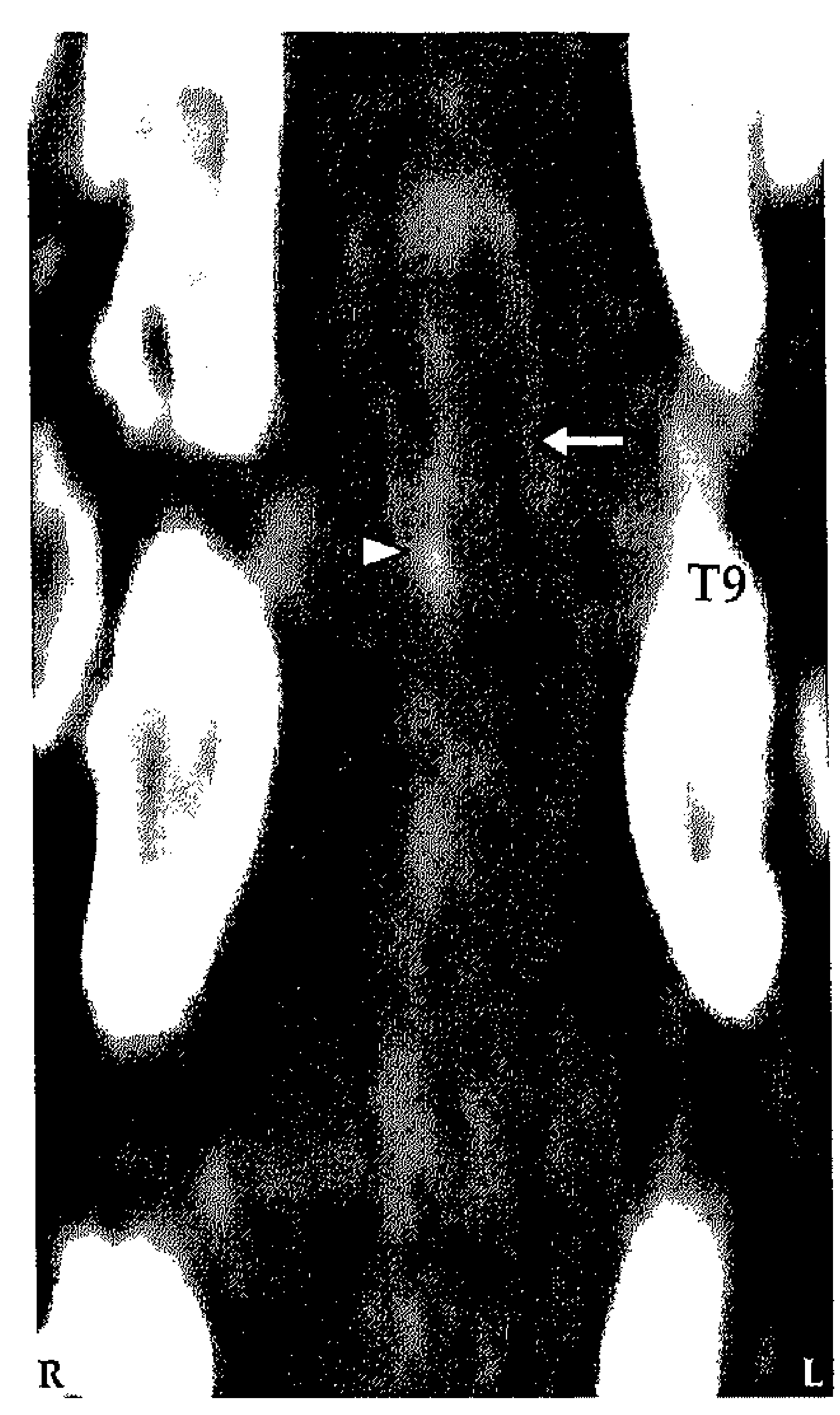

a

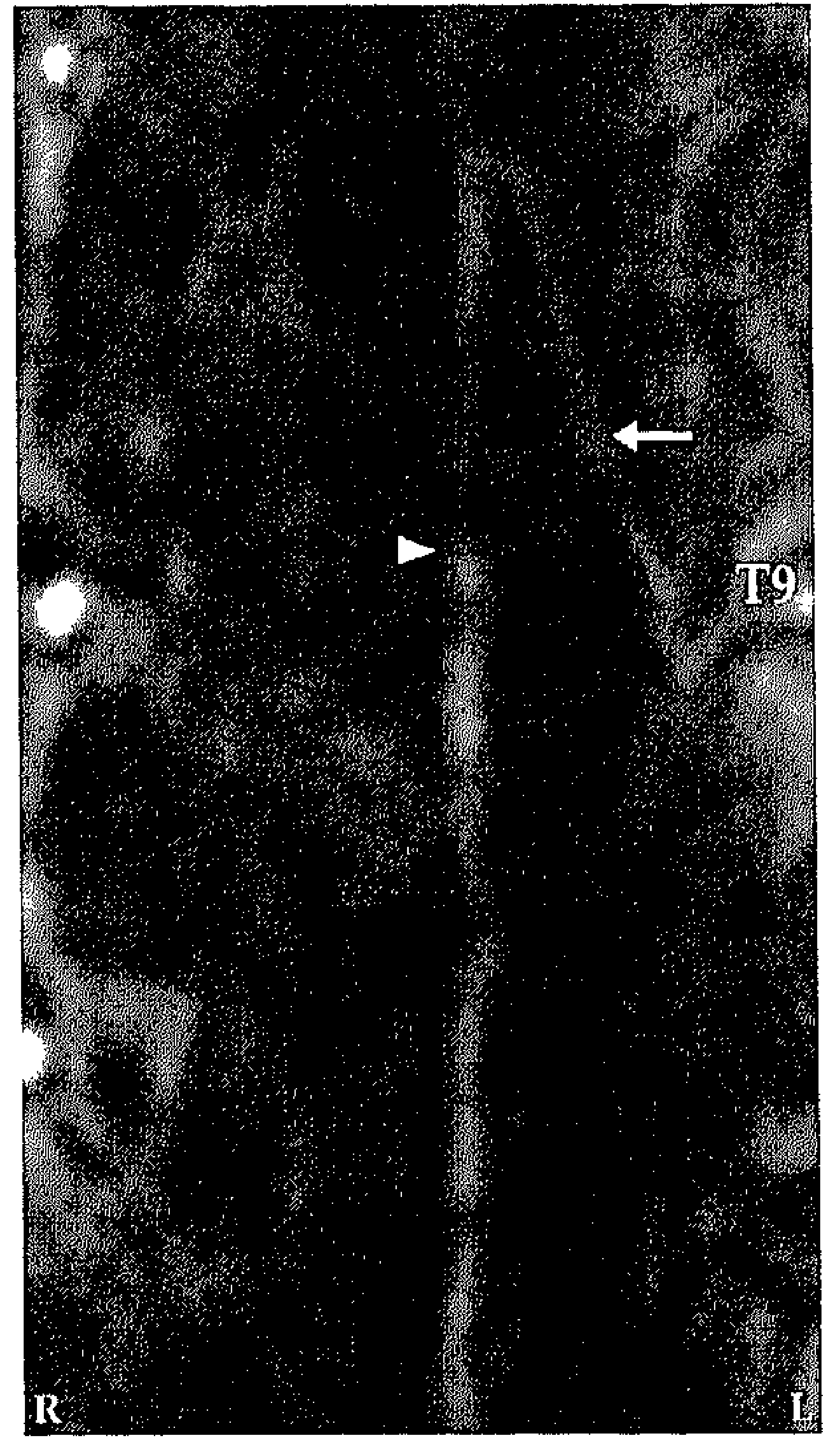

b

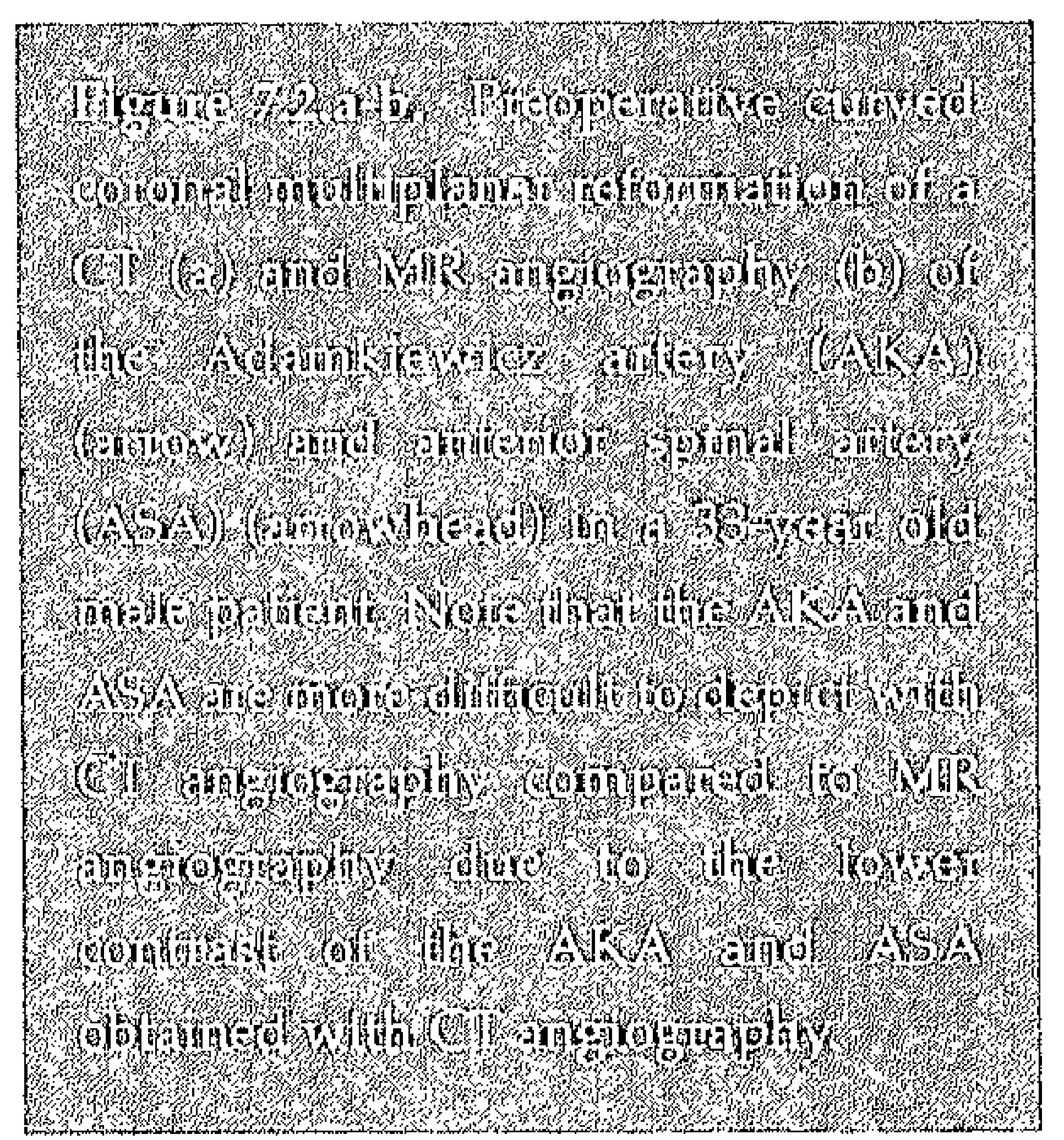

signal changes between the first and second phase MR angiography images in these 36 cases. In 3 patients no GARV could be depicted using MR angiography, but spinal cord drainage via the filum vein could be detected.

Using CT angiography the AKA could be more often localized employing $80 \mathrm{kV}$ tube voltage $(77 \%$ (20/26)) compared to $120 \mathrm{kV}$ $(31 \%(4 / 13))$. In 8 cases none of the observers was able to detect the AKA on CT angiography images. There were 7 cases in which only one of the observers was able to detect the AKA on CT angiography images. The percentage of interobserver agreement for $\mathrm{CT}$ angiography was $82 \%$. For MR angiography there were two cases in which only one observer was unable to localize the AKA.
Using MR angiography the interobserver agreement was $94 \%$.

Observational image quality. Contrast of the AKA versus the adjacent innage region $(P<0.001)$ and overall image quality $(P<0.004)$ were judged to be significantly better for MR angiography (Table 7.3). Spinal cord tissue enhancement was evaluated to be significantly $(P<0.03)$ stronger for $C T$ angiography. Epidural venous enhancement was judged to be significantly $(P<0.001)$ less present on $C T$ angiography images. There was no significant $(P>0.7)$ difference in image noise between the two modalities.

Quantitative image quality. Quantitative image analysis revealed that SNR of the AKA was significantly higher $(P<0.001)$ for $C T$ angiography (62.2 \pm 18$)$ compared to $M R$ 
Table 7.2. Overview of the localizations and frequencies of the Adamkiewicz artery (AKA) and great anterior radiculomedullary vein (GARV) as detected by $M R$ and $C T$ angiography.

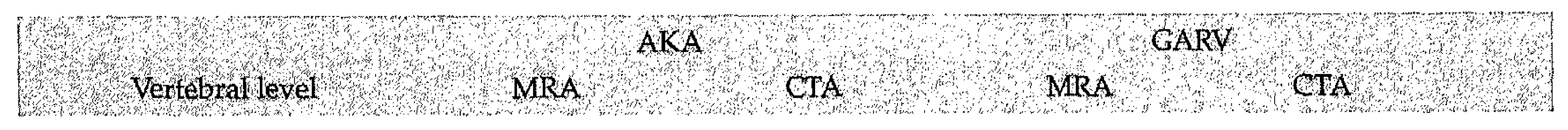

$\begin{array}{ccccc}\text { T8 } & 2 & 2 & 0 & 0 \\ \text { T9 } & 9 & 4 & 0 & 0 \\ \text { T10 } & 13 & 11 & 1 & 0 \\ \text { T11 } & 8 & 7 & 1 & 0 \\ \text { T12 } & 6 & 6 & 5 & 1 \\ \text { L1 } & 1 & 1 & 9 & 7 \\ \text { L2 } & 0 & 0 & 11 & 9 \\ \text { L3 } & 0 & 0 & 8 & 1 \\ \text { L4 } & 0 & 0 & 1 & 1\end{array}$

Reported are the vessels that were detected by at least one of the two observers. No AKA or GARV was detected outside the indicated vertebral levels. Note that the GARV is generally more caudally localized than the AKA.

Table 7.3. Observational (subjective) image quality comparison between CT and MR angiography,

\begin{tabular}{|c|c|c|c|}
\hline Contrast AKA versus surrounding & $1.9 \pm 1.3$ & $3.1 \pm 1.0$ & $P<0.001^{*}$ \\
\hline Image noise & $3.5 \pm 0.7$ & $3.5 \pm 0.8$ & $P>0.7$ \\
\hline Spinal cord enhancement & $3.5 \pm 0.7$ & $3.2 \pm 0.8$ & $P<0.03^{*}$ \\
\hline Epidural venous enhancement & $2.1 \pm 0.7$ & $2.6 \pm 0.7$ & $P<0.001^{*}$ \\
\hline Overall image quality & $2.8 \pm 0.8$ & $3.2 \pm 0.6$ & $P<0.004^{*}$ \\
\hline
\end{tabular}

Note: * indicates statistically significant. Values are presented as mean \pm SD. 

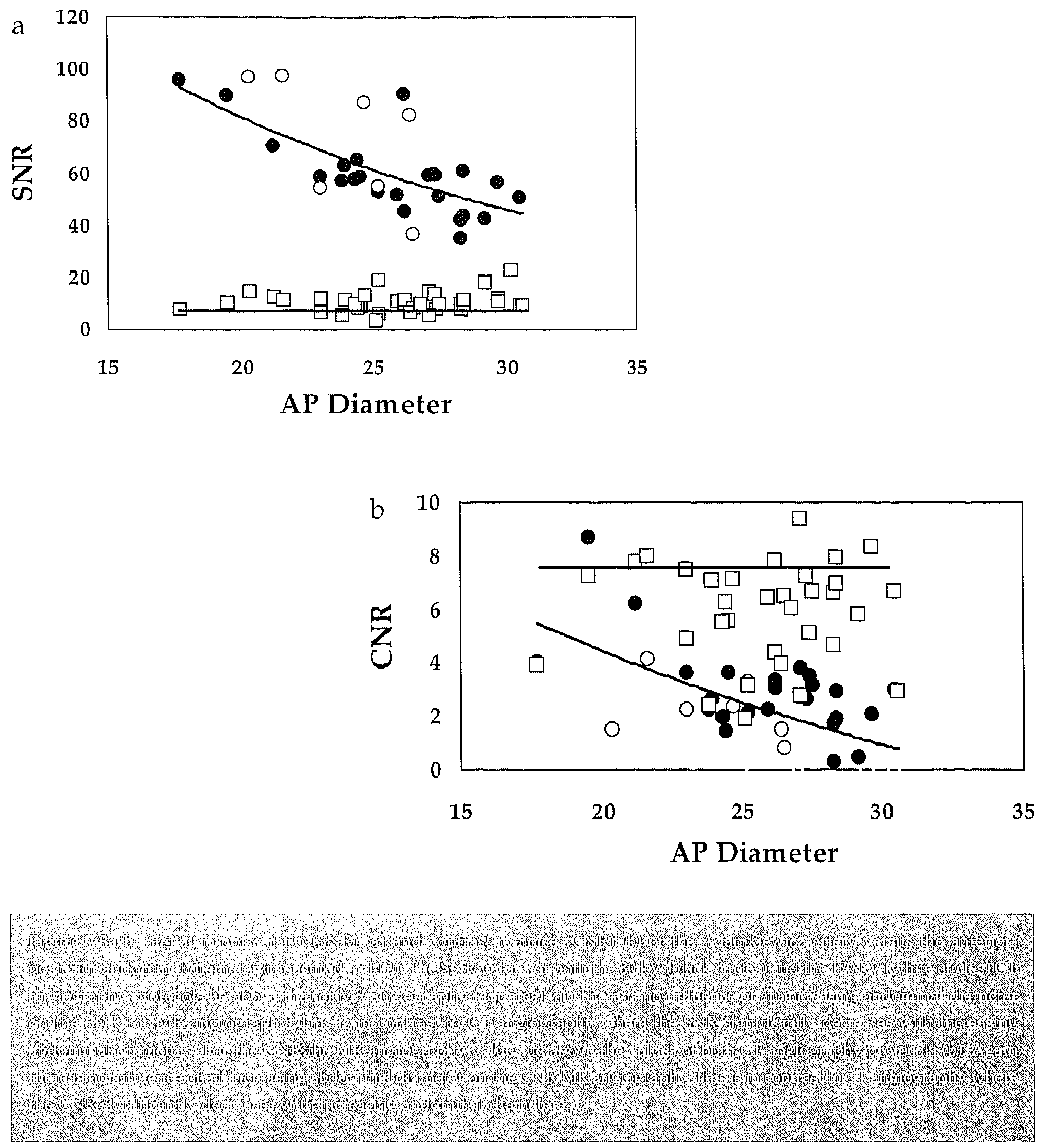
Table 7.4. Quantitative (objective) image quality comparison between $\mathrm{CT}$ and $\mathrm{MR}$ angiography.

\begin{tabular}{|c|c|c|c|c|}
\hline SNR & $59 \pm 15$ & $73 \pm 24$ & $10.4 \pm 3.2$ & $P<0.001^{*}$ \\
\hline CNR & $3.0 \pm 1.7$ & $2.3 \pm 1.1$ & $6.8 \pm 2.4$ & $P<0.001^{*}$ \\
\hline
\end{tabular}

Note: SNR indicates signal-to-noise ratio; CNR, contrast-to-noise ratio, ${ }^{*}$ statistically significant. Values are presented as mean $\pm \mathrm{SD}$.

angiography (10.4 \pm 3.2 ) (Table 7.4). The signal intensity of the image region adjacent to the AKA was not significantly $(P>0.5)$ different for $\mathrm{CT}$ angiography $(14.8 \pm 22.4)$ compared to $\mathrm{MR}$ angiography $(13.0 \pm 11.1)$. The $\mathrm{CNR}$ of the AKA was significantly $(P<0.001)$ higher for MR angiography $(6.8 \pm 2.4)$ than for $C T$ angiography $(2.8 \pm 1.6)$ (Table 7.4). At CT angiography, both SNR $(P<0.001)$ and $C N R$ $(P<0.01) \quad$ decreased significantly with increasing patient diameter. At MR angiography, there was no significant relation between SNR $(P>0.3)$ or CNR $(P>0.6)$ and patient diameter (Figure 7.3).

\section{Clinical outcome}

No intraoperative death or renal failure occurred in this series of patients. After surgery, five patients $(13 \%)$ died in the hospital: one patient with a TAA (respiratory failure), two patients with a type II TAAA (cardiac and respiratory failure), one patient with a type III TAAA (sepsis and respiratory failure), and one patient with a type IV TAAA (sepsis). Two patients developed paraplegia.

In one patient (type III TAAA), acute paraplegia became evident after the procedure. This neurologic deficit was anticipated because motor evoked potentials (MEPs) were entirely absent at the end of the procedure. In this patient sequential clamping of the thoracic and abdominal aorta was associated with a gradual decrease of MEPs to non-measurable amplitudes. Despite increasing mean arterial and distal aortic pressures and revascularization of all available segmental arteries, including the one supplying the AKA, MEP signals did not return.

In one type II TAAA patient, delayed paraplegia occurred. In this patient, exclusion of the entire aorta did not lead to a decline in MEPs. Because of the very poor quality of the aorta and because no MEP declines were observed during the entire operation, no attempts were undertaken to revascularize any of the encountered back-bleeding segmental arteries, including the supplier of the AKA. At the intensive care unit, the patient experienced a short period of hypotension. Thereafter, he was unable to move his legs.

\section{DISCUSSION}

This study investigated whether MR or CT angiography is the best technique to visualize the AKA in Caucasian TAAA patients. The higher AKA detection rate revealed that MR angiography performed better than $\mathrm{CT}$ angiography in our TAAA patient population. The superiority of MR angiography was 
confirmed by both quantitative and observational image quality analysis.

Despite recent advances in surgical techniques and intraoperative monitoring paraplegia remains a feared and devastating complication after technically successful TAAA repair. Preoperative identification of the AKA and its segmental supplier in TAAA patients has been suggested as a protective measure as it provides the opportunity to selectively reattach the spinal cord blood supplying arteries. The imaging technique used to detect to the arterial trajectory to the AKA should ideally have a high detection rate, be safe to perform, patient friendly, and unambiguous regarding the interpretation what is inlet artery or outlet vein of the spinal cord. In general, the standard of reference for spinal cord angiography is catheter angiography. Although this technique may provide superior image quality it is not recommended in TAAA patients at our institutes. Even when performed by specialists catheter angiography in TAAA patients may be associated with severe complications, including paraplegia1. Manoeuvring the catheter through the atherosclerotic aorta and hooking the tip of the catheter into the segmental arteries may induce emboli and subsequent occlusion by disrupting atherosclerotic plaques. Furthermore injection into and manoeuvring of the catheter in the segmental artery can lead to vasospasm and temporary occlusion of these segmental arteries 21 .

\section{$C T$ versus $M R$ angiography}

Recently non-invasive imaging modalities such as $C^{3,4,9,16}$ and MR angiography 5-9,13-18 have been explored to assess their potential to non-invasively localize the AKA. Both imaging modalities seem capable of visualizing the AKA and its segmental supply. Reported detection rates of the AKA vary from 68 to $90 \%$ for CT angiography 3,4 and between 67 to $100 \%$ for MR angiography $5,6,9$.

We found that MR angiography was superior to CT angiography in all important metrics including AKA detection rate $(97 \%$ versus $71 \%)$, interobserver agreement (94\% versus $82 \%$ ) and image quality (score 3.2 versus 2.8). Differences between our results and those of Japanese groups $3,4,9,16$ may be explained by differences in image quality related to the exact implementation of the imaging techniques and patient populations.

Contrast appears to be the most relevant image quality item to detect the AKA. Visibility of small structures, like spinal cord arteries (caliber $<1.0 \mathrm{~mm}$ ), is dependent on the contrast between vessel lumen and surrounding. This was also noticed in the quantitative (in terms of CNR) and observational assessment of the images of the two modalities. MR angiography was judged to be significantly better regarding contrast between the AKA and the surrounding tissue with significantly less spinal cord tissue enhancement (darker background).

For CT angiography, the contrast between the iodine containing vessel and surrounding is dependent on several factors. First of all, tube voltage $(\mathrm{kV})$ is important as lower voltages will lead to stronger attenuation by intravascular contrast material compared to a higher voltage 22 . Stronger attenuation in the vessel lumen gives rise to more contrast and thus improved visualization. This was reflected by the more frequent $\mathrm{AKA}$ detection in the (sub) group of patients who were scanned with the lower $\mathrm{kV}$ setting. Secondly, the body habitus of the patient plays a crucial 
role. It was found that CNR decreases significantly for patients with larger abdominal AP-diameters. This can be explained by the fact that in corpulent individuals X-rays will be absorbed more strongly by the (thicker) body resulting in less $X$-ray photons transmitted through the body to the detectors. This consequently causes more noise and lower SNR and CNR values ${ }^{22}$. Since our Caucasian patient population is not surprisingly more obese than the Japanese the results of those studies may not apply to the current Caucasian patient population.

Apart from the current results in favor of MR angiograhy, it should be recalled that MRI does not involve ionizing radiation. Performing thoracoabdominal CT angiography is associated with a relatively high effective dose of approximately 20 milliSievert, which is relatively high for diagnostic imaging. Moreover, gadolinium-based MR contrast agents as used in the current study have a more favorable safety profile with a much lower frequency of renal complications in vascular comprised patient populations compared to the iodine based contrast agents used for CT angiography.

A limitation of the current study is that we did not use a dedicated timing in our CT protocol, as opposed to MR angiography. However, due to the complex shape of the aortic aneurysms timing in CT angiography is difficult regardless if a fixed scan delay or an automated trigger system is used. In addition one might argue that the CT-scanner used in this study is not state-of-the-art, when compared to the current generation of 16 and 64 slice machines. More detectors would allow faster imaging, possibly acquiring more data at the peak of the contrast enhancement. Recently, Boll et $\mathrm{al}^{23}$ used a 40 slice CT system to visualize the AKA in 100 (non-vascular) patients with a pancreatic neoplasm and achieved acquisition times of approximately 7 seconds. Although these authors claimed an AKA detection rate of $100 \%$, spinal cord feeding arteries were not differentiated from draining veins. Definite classification of spinal vessels in terms of arterial and venous was not validated and remains highly uncertain in our opinion. Therefore it remains to be proven whether this advanced technology will improve the AKA visualization in vascular patients. Moreover, Takase et $\mathrm{al}^{4}$ used an identical 4-slice CT scanner and found a AKA detection rate of $90 \%$ in a group of 70 Japanese vascular diseased patients.

\section{Implications for presurgical work-up}

At our institutions, and probably in most other hospitals, CT angiography is used to determine the extent, diameter, and type (atherosclerotic or dissection) of the aortic aneurysm. Furthermore, CT angiography is also logistically more easily accessible than MR angiography.

In case CT angiography, preferably performed at $80 \mathrm{kV}$ tube voltage for corpulent patients, is able to unambiguously detect the AKA and its segmental supply it would a priori be the preferred and only technique to be applied in the presurgical work-up. However, this study showed that MR angiography outperforms CT angiography on the AKA localization. Besides the lower AKA detection ratio, CT angiography is not (yet) able to unambiguously differentiate between the AKA and the outlet vein. In our opinion differentiation, i.e., answering what is inlet artery (i.e., AKA) and outlet vein (i.e., GARV), is of paramount importance. At present only MR angiography can differentiate between 
spinal cord inlet artery and outlet vein.

In consideration of the foregoing, overall surgical planning should involve CT angiography to detect and characterize the aortic aneurysm. When CT angiography does not detect the AKA or when the continuity of the blood supplying arterial trajectory from the aorta to the spinal cord cannot indisputably be visualized, because of insufficient image quality, we suggest to perform MR angiography to identify the AKA.

In conclusion, non-invasive assessment of spinal cord blood supply in Caucasian TAAA patients is feasible with both $C T$ and MR angiography. Because the detection rate of MR angiography and image quality is superior to $\mathrm{CT}$ angiography we conclude that MR angiography is the preferred modality in Caucasian TAAA patients.

\section{Acknowledgement}

We are grateful to dr. G.K. Kemerink for his advice and comments on the manuscript. 


\section{REFERENCES}

1. Kieffer E, Fukui S, Chiras I, Koskas F, Bahnini A Cormier E. Spinal cord arteriography: a safe adjunct before descending thoracic or thoracoabdominal aortic aneurysmectomy. J Vasc Surg 2002;35:262-8.

2. Williams GM, Roseborough GS, Webb TH, Perler BA Krosnick T. Preoperative selective intercostal angiography in patients undergoing thoracoabdominal aneurysm repair. J Vasc Surg 2004;39:314-21.

3. Kudo K, Terae S, Asano T, et al. Anterior spinal artery and artery of Adamkiewicz detected by using multidetector row CT. AJNR Am J Neuroradiol 2003;24:13-7.

4. Takase K, Sawamura Y, Igarashi K, et al. Demonstration of the artery of Adamkiewicz at multidetector row helical CT. Radiology 2002;223:39-45.

5. Nijenhuis RI, Leiner T, Cornips EM, et al. Spinal cord feeding arteries at MR angiography for thoracoscopic spinal surgery: feasibility study and implications for surgical approach. Radiology 2004;233:541-7.

6. Nijenhuis RJ, Gerretsen S, Leiner T, Jacobs MJ, van Engelshoven JM, Backes WH. Comparison of 0.5-M Gd-DTPA with 1.0-M gadobutrol for magnetic resonance angiography of the supplying arteries of the spinal cord in thoracoabdominal aortic aneurysm patients. J Mngn Reson Imaging 2005;22:136-44.

7. Hyodoh $\mathrm{H}$, Kawaharada $\mathrm{N}$, Akiba $\mathrm{H}$, et al. Usefulness of preoperative detection of artery of Adamkiewicz with dynamic contrast-enhanced MR angiography. Radiology 2005;236:1004-9.

8. Yamada N, Okita Y, Minatoya K, et al. Preoperative demonstration of the Adamkiewicz artery by magnetic resonance angiography in patients with descending or thoracoabdominal aortic aneurysms. Eur J Cardiothorac Surg 2000;18:104-11.

9. Yoshioka $K$, Niinuma $H$, Ohira $A$, et al. MR angiography and $\mathrm{CT}$ angiography of the artery of Adamkiewicz: noninvasive preoperative assessment of thoracoabdominal aortic aneurysm. Radiographics 2003;23:1215-25.
10. Thron A. Anatomy of the spinal cord's blood supply. In: Thron A, ed. Vascular anatomy of the spinal cord. Wien: Springer-Verlag; 1988:8-12.

11. Thron A. Vascular anatomy of the spine. In: Byrne J, ed. Interventional Neuroradiology. Oxford: Oxford University Press; 2002:19-23.

12. Jacobs MJ, Mess W, Mochtar B, Nijenhuis RJ, Statius van Eps RG, Schurink GW. The value of motor evoked potentials in reducing paraplegia during thoracoabdominal aneurysm repair. I Vasc Surg 2006;43:239-46.

13. Kawaharada $\mathrm{N}$, Morishita $\mathrm{K}$, Hyodoh $\mathrm{H}$, et al. Magnetic resonance angiographic localization of the artery of Adamkiewicz for spinal cord blood supply. Am Thorac Surg 2004;78:846-51; discussion 51-2.

14. Nijenhuis RJ, Mull M, Wilmink JT, Thron AK, Backes WH. MR angiography of the great anterior radiculomedullary artery (Adamkiewicz artery) validated by digital subtraction angiography. $A J N R$ Am I Neuroradiol 2006;27:1565-72.

15. Nijenhuis RJ, Jacobs MJ, van Engelshoven JM, Backes WH. MR angiography of the Adamkiewicz artery and anterior radiculomedullary vein: postmortem validation. AJNR Am J Neuroradiol 2006;27:1573-5.

16. Yoshioka $K$, Niinuma $H$, Ehara $S$, Nakajima $T$, Nakamura M, Kawazoe K. MR angiography and CT angiography of the Artery of Adamkiewicz: State of the Art. Radiographics 2006;26 Suppl 1:S63-73.

17. Kawaharada N, Morishita K, Fukada J, et al. Thoracoabdominal or descending aortic aneurysm repair after preoperative demonstration of the Adamkiewicz artery by magnetic resonance angiography. Eur J Cardiothorac Surg 2002;21:970-4.

18. Yamada N, Takamiya M, Kuribayashi S, Okita $Y$, Minatoya K, Tanaka R. MRA of the Adamkiewicz artery: a preoperative study for thoracic aortic aneurysm. J Comput Assist Tomogr 2000;24:362-8. 
19. Jacobs MJ, Elenbaas TW, Schurink CW, Mess WH, Mochtar B. Assessment of spinal cord integrity during thoracoabdominal aortic aneurysm repair. AnI Thorac Surg 2002;74:S1864-6; discussion S92-8.

20. Jacobs MJ, Mess WH. The role of evoked potential monitoring in operative management of type $I$ and type II thoracoabdominal aortic aneurysms. Semin Thorac Cardiowase Surg 2003;15:353-64.

21. Forbes G, Nichols DA, Jack CR, Jr., et al. Complications of spinal cord arteriography: prospective assessment of risk for diagnostic procedures. Radiology 1988;169:479-84.

22. Nakayama $Y$, Awai K, Funama Y, et al. Abdominal CT with low tube voltage: preliminary observations about radiation dose, contrast enhancement, image quality, and noise. Radiology 2005;237:945-51.

23. Boll DT, Bulow H, Blackham KA, Aschoff AJ, Schmitz $B L$. MDCT angiography of the spinal vasculature and the artery of Adamkiewicz. AIR Am J Rocntgenol 2006;187:1054-60. 


\section{Chapter VIII}

MR angiography and neuromonitoring to assess spinal cord blood supply in thoracic and thoracoabdominal aortic aneurysm surgery

RJ Nijenhuis MJ Jacobs GW Schurink ACH Kessels MA van Engelshoven WH Backes 


\section{ABSTRACT}

Purpose: Preoperative knowledge of the bloodsupplying trajectory to the spinal cord is of interest, because spinal cord ischemia may occur during thoracic aortic aneurysm (TAA) and thoracoabdominal aortic aneurysm (TAAA) repair and possibly leads to paraplegia. The Adamkiewicz artery (AKA) is considered to be the most important blood supplier of the thoracolumbar spinal cord and has therefore been the focus in preoperative diagnostic imaging. However, in $\operatorname{TAA}(\mathrm{A})$ patients, the blood supply to the spinal cord may strongly depend on (intersegmental) collateral circulation, because many segmental arteries are occluded as a result of atherosclerosis. Therefore, the importance of preserving the segmental artery supplying the AKA (SAAKA) is debated. Here it was investigated whether (i) the AKA and its segmental supplier can be imaged by using magnetic resonance (MR) angiography and (ii) aortic cross-clamping of the SA-AKA influences intraoperative spinal cord function, monitored by motor evoked potentials (MEPs).

Materials and Methods: Preoperative MR angiography was performed to localize the SA-AKA and the AKA in 60 patients (19 TAA, 7 TAAA I, 18 TAAA II, 9 TAAA III, and 7 TAAA IV). Spinal cord function was monitored during surgery by using MEPs. When MEPs indicated critical ischemia, the SA-AKA was selectively reattached. To test whether aortic cross-clamping of the SA-AKA was associated with MEP decline, the Fisher statistical exactness test was applied.

Results: The AKA and SA-AKA could be localized in all $60(100 \%)$ patients between vertebral levels T8 and L2 (72\% left-sided). In $44(73 \%)$ patients, the SA-AKA was cross- clamped, which led in $32 \%(14 / 44)$ of cases to MEP decline. Reattachment of the preoperatively localized SA-AKA re-established MEPs and, thus, spinal cord function in 12 of 14 cases. When the SA-AKA was outside the area cross-clamped, the MEPs always remained stable. A significant association $(P<0.01)$ was found between the location of the SA-AKA relative to the aortic cross-clamps and the MEPs.

Conclusions: The AKA can be localized before surgery in $100 \%$ of TAA(A) patients by using MR angiography. Location of the SAAKA outside the cross-clamped aortic area is attended with stable MEPs. Interestingly, it was found that in most patients in whom the SA-AKA was cross-clamped, MEPs were not affected, thus indicating sufficient collateral blood supply to maintain spinal cord integrity. Nevertheless, preoperative knowledge of SAAKA location is of importance, because in $32 \%$ of patients, spinal cord function was dependent on this supplier. Revascularization of the SA-AKA can thereby reverse spinal cord dysfunction. 


\section{INTRODUCTION}

In thoracic aortic aneurysm (TAA) and thoracoabdominal aortic aneurysm (TAAA) repair, maintenance of spinal cord integrity remains difficult and challenging. The blood supply to the spinal cord must be assured, because spinal cord injury in TAA(A) surgery may result even from temporary ischemia. Under normal physiological conditions, the anterior spinal artery (ASA) of the thoracolumbar spinal cord is supplied by several (1-4) anterior radiculomedullary arteries 1,2 . The largest (and, therefore, thought to be the most important supplier) is known as the Adamkiewicz artery (AKA). The vertebral level of the segmental artery (SA) supplying the AKA may, however, differ among individuals 1,2 . Normally the blood supplying pathway to the spinal cord is from the aorta through the SA, the AKA, and finally the ASA. The pathway through the $S A$ at the level of the AKA is referred to as being the direct spinal cord supply. In TAA(A) patients, however, the SA at the level of the AKA may be occluded at its origin in the aortic wall because of the presence of atherosclerosis. The spinal cord then becomes dependent on intersegmental collateral supply ${ }^{3-5}$. This is referred to as being the indirect spinal cord supply.

Because multiple blood supplying trajectories to the spinal cord seem to exist, there are opposite views either for ${ }^{6,7}$ or against ${ }^{8-10}$ preoperative localization of the SA supplying the AKA and its revascularization. Visualization of the spinal cord arteries by means of intra-arterial catheter (X-ray) angiography, also known as digital subtraction angiography, which is the standard of reference, is not a straightforward procedure. Moreover, catheter angiography is associated with a highly variable sensitivity $(43-86 \%)^{4,11}$ nephrotoxic contrast agent, and iatrogenic paraplegia $(0.2 \%)^{4}$. Therefore, preoperative localization of the AKA by using catheter angiography is not performed in most preoperative workup programs.

Although preoperative imaging can visualize the blood supply to the spinal cord, it cannot provide information on its (intraoperative) functionality. To monitor the spinal cord function during surgery, the technique of transcranial motor evoked potentials (MEPs) can be used5,12,13. However; despite the use of MEPs in addition to other intraoperative protective measures, postoperative paraplegia still occurs ${ }^{12}$.

Because of technical developments in noninvasive imaging modalities such as computed tomographic (CT) and magnetic resonance (MR) angiography, it has now become possible to visualize the arteries responsible for the blood supply to the spinal cord in a non-invasive manner ${ }^{14-19}$. Preoperative MR angiography studies of the spinal cord blood supply performed so far have studied the prevalence of postoperative paraplegia7,16,20,21. In these studies, several intraoperative neuroprotective measures, except the use of intraoperative functional monitoring (e.g., MEPs), were applied. These studies showed different outcomes regarding the benefit of preoperative localization of the segmental supplier of the AKA. However, these studies did not demonstrate whether the SA supplying the AKA was crucial to maintain the spinal cord function. In our opinion, the question of whether a SA supplying the AKA is crucial or not can be answered only by relating the aortic cross-clamping of this SA to functional measures of the spinal cord, such as MEPs. A combined approach of preoperative 
angiography with intraoperative spinal cordderived MEPs and segmental cross-clamping would allow investigation of the functionality of the SA supplying the AKA and the relevance for localizing them.

If a relationship between the preoperative MR angiographic localization of the SA supplying the AKA relative to the aortic crossclamping area and the corresponding MEP signal can be found, this might have implications for surgical strategies, both endovascular and open. Therefore, it was investigated whether (i) the AKA and its segmental supplier can be imaged by using MR angiography in aortic aneurysm patients and (ii) aortic cross-clamping of the SA supplying the AKA influences intraoperative spinal cord function.

\section{MATERIALS and METHODS}

\section{Patients}

From September 2002 to October 2005, 60 patients ( 32 male and 28 female) with either a TAA or TAAA who planned to undergo elective surgical aortic aneurysm repair were included in this study. This series was not consecutive because patients whose conditions were too critical to undergo MR angiography examination at the time of admission were not included. The 60 patients (age, $66 \pm$ 11 years; mean \pm SD) were divided into 5 different groups according to the extent of their aortic pathology by using the Crawford classification: 19 TAA, 7 TAAA type I, 18 TAAA type II, 9 TAAA type III, and 7 TAAA type IV. Of the 60 included patients, $21(35 \%)$ had a chronic type $B$ dissection and, to some extent, atherosclerosis. The remaining 39 patients had advanced atherosclerotic disease.
On the basis of the pathologic features, the 60 patients were divided into the dissection group (21 patients) or the atherosclerotic group (39 patients).

The medical ethics committee of the hospital approved the protocol. Written, informed consent was obtained from all patients before their inclusion.

\section{$M R$ angiography}

All patients underwent preoperative MR angiography to localize the AKA and its segmental supplier. Imaging was performed on a clinical 1.5-Tesla MR imaging system (Philips Intera; Philips Medical Systems, Best, The Netherlands) equipped with a phasedarray synergy surface spine coil. The MR imaging protocol included a bolus timing scan series, in which the time of contrast arrival in the distal aorta was determined. Subsequently, the MR angiography examination (consisting of two consecutive dynamic phases) was performed by using a threedimensional spoiled gradient echo sequence. A centric k-space filling scheme synchronized to the arrival of the contrast agent (dose 0.3 $\mathrm{mmol} / \mathrm{kg}$ ) was used to suppress the venous blood signal. Voxel sizes were $0.8 \times 0.8 \times 1.2$ $\mathrm{mm}$ at acquisition. The two dynamic phases allowed differentiation based on temporal intensity changes between the AKA and the great anterior radiculomedullary vein (GARV), which has a similar spatial configuration. Details of the imaging protocol have been described elsewhere ${ }^{14,15}$.

\section{Image analysis}

MR angiography images were postprocessed by using curved multiplanar reformation. One image creator determined the level and side of the AKA and its segmental 
supplier according to criteria described elsewhere ${ }^{14}$. When the SA directly connecting to the AKA was occluded at its origin in the aortic wall, the nearest open SA with an intersegmental connection to the (partially) occluded SA was suggested to be the segmental supplier of the AKA. This situation was referred to as being the indirect supply to the spinal cord. Because many SAs are occluded due to atherosclerosis, the number of SAs with an open (i.e., direct) connection to the aorta were determined by MR angiography for every patient to investigate whether there was a difference between the dissection and atherosclerotic group. Localization of the AKA and its segmental supplier could be performed within 15 minutes. Before the operation, the localization of the segmental supplier of the AKA was reported to the surgeon.

\section{Surgical protocol}

The surgical procedure was performed in manner similar to that described previously ${ }^{5,12}$. Our spinal cord protection protocol consisted of cerebrospinal fluid (CSF) drainage, left atrium-femoral artery or femoral artery-femoral vein bypass enabling distal aortic perfusion, and spontaneous cooling to $32^{\circ} \mathrm{C}$ to $33^{\circ} \mathrm{C}$ with active rewarming at the end of the procedure. For all patients, the aortic cross-clamp positions, subsequent MEP signals, localization of open (i.e., backbleeding) SAs, and revascularization or oversewing of a SA were registered during the operation and written in the operation report. The surgeon determined the level of the clamp by counting vertebral bodies upward or downward from vertebral level T12.

\section{MEP monitoring and subsequent operative strategies}

The technique of transcranial stimulation has been described in detail elsewhere ${ }^{12,13,22}$. In general, the aorta is sequentially crossclamped, thus allowing stepwise exclusion of aortic segments and assessment of changes in MEP amplitudes. If after placement of the proximal clamp the MEPs decreased, the mean distal aortic perfusion pressure was increased until the MEPs normalized. However, if during aortic cross-clamping the MEPs rapidly decreased and were not correctable with increasing distal and mean arterial pressure, thus indicating that the excluded aortic segment contained crucial SAs, the clamps were released, and the patient was actively cooled to $32^{\circ} \mathrm{C}$, thus affording additional neuroprotection. The aorta was then clamped again and opened. Patent SAs were reattached and perfused until the MEPs returned to baseline levels. When the origin of the SA supplying the AKA was inside the cross-clamped area, this SA was reattached and perfused first. In case MEP amplitudes remained stable, some SAs were preventively reattached, except when they arose from a severely diseased aortic wall. When possible, revascularization of the hypogastric arteries was additionally performed.

\section{Combining $M R$ angiography with $M E P S$}

After the operation, the localization of the segmental supplier of AKA on MR angiography was related to the cross-clamp positions and the corresponding MEP signals. Because the localization of the segmental supplier to the AKA was either inside or outside the cross-clamped area, two different groups of patients were constituted. 


\section{Statistical analysis}

The Fisher statistical exactness test was performed to test whether aortic crossclamping of the SA supplying the AKA was associated with MEP decline. The positive and negative predictive values, including confidence intervals, were calculated to demonstrate the relevance for localizing the SA supplying the AKA with respect to the cross-clamped aorta area and the MEP signal. To determine whether the number of open preoperatively determined SAs was significantly different between the atherosclerotic and dissection groups, the Wilcoxon signed rank test was used. To investigate whether there was a significant difference in direct or intersegmental supply of the AKA between the atherosclerotic and dissection group, the Fisher statistical exactness test was also used. Statistical significance was inferred when the obtained $P$-value was $<0.05$.

\section{RESULTS}

\section{$M R$ angiography}

Adequate MR angiography was obtained in all $60(100 \%)$ TAA(A) patients. No adverse effects occurred during or after contrast injection. Although partial enhancement of the GARV was present in the first phase in 46 of the 60 patients, the AKA could be separated from the GARV and visualized in all 60 patients in the first phase images (example in Figure 8.1). The GARV was depicted in 48 patients and could be localized best in the second phase images.

The arterial trajectory from the aorta through the SA and AKA to the ASA could be depicted in all 60 patients (example in Figure 8.2). However, the SA directly connecting to

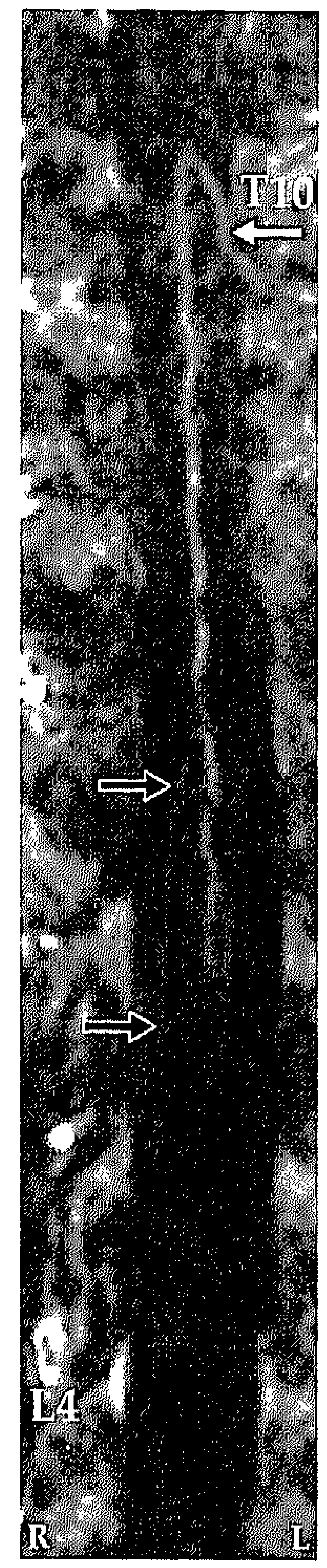

a

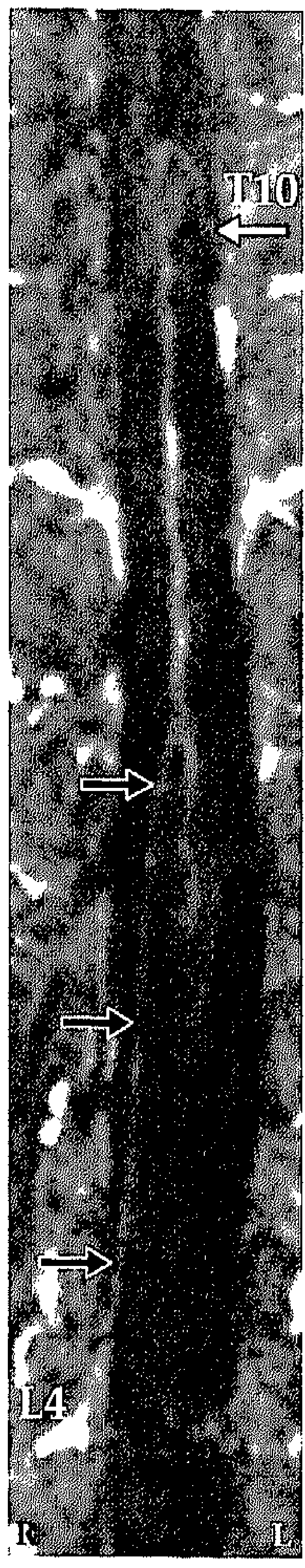

b

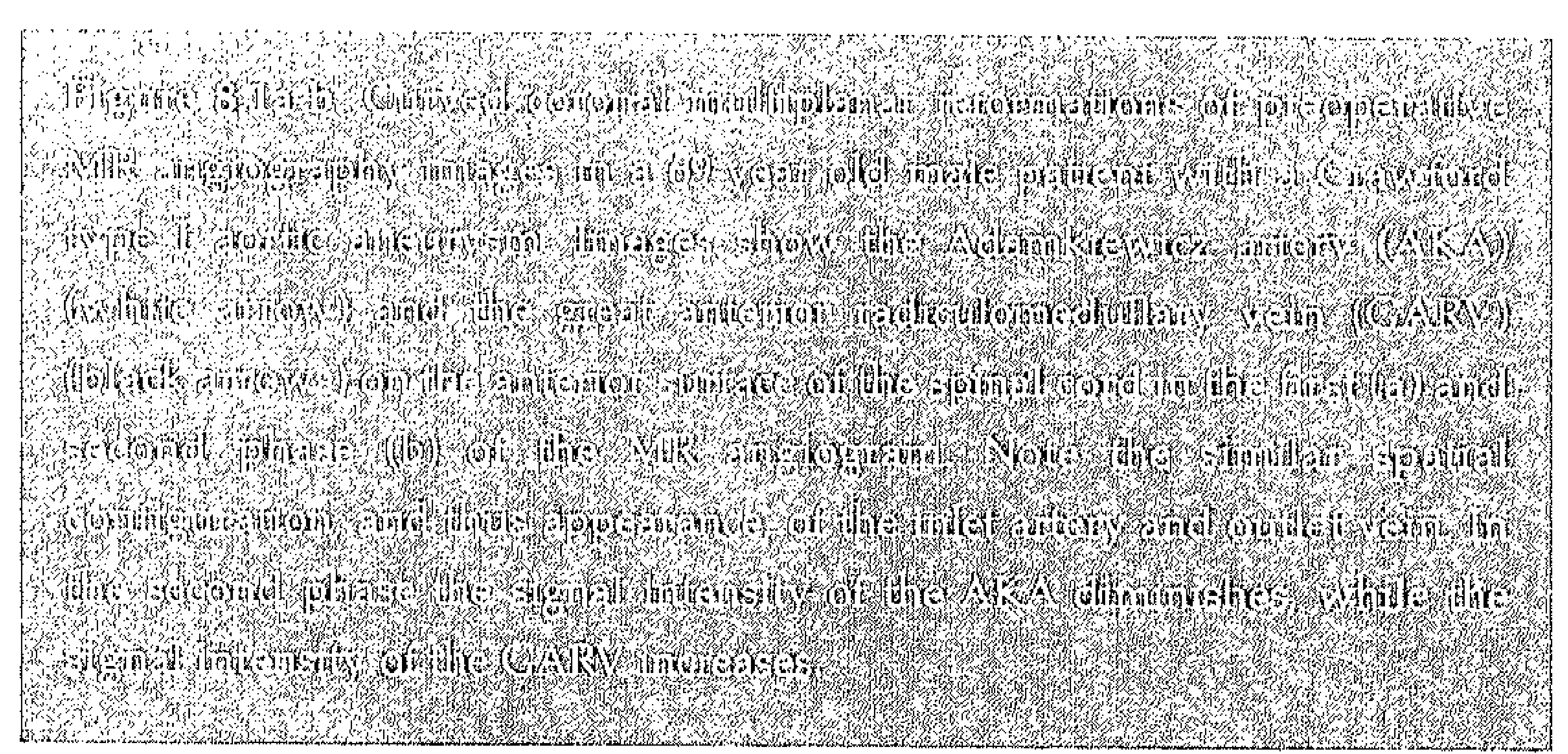



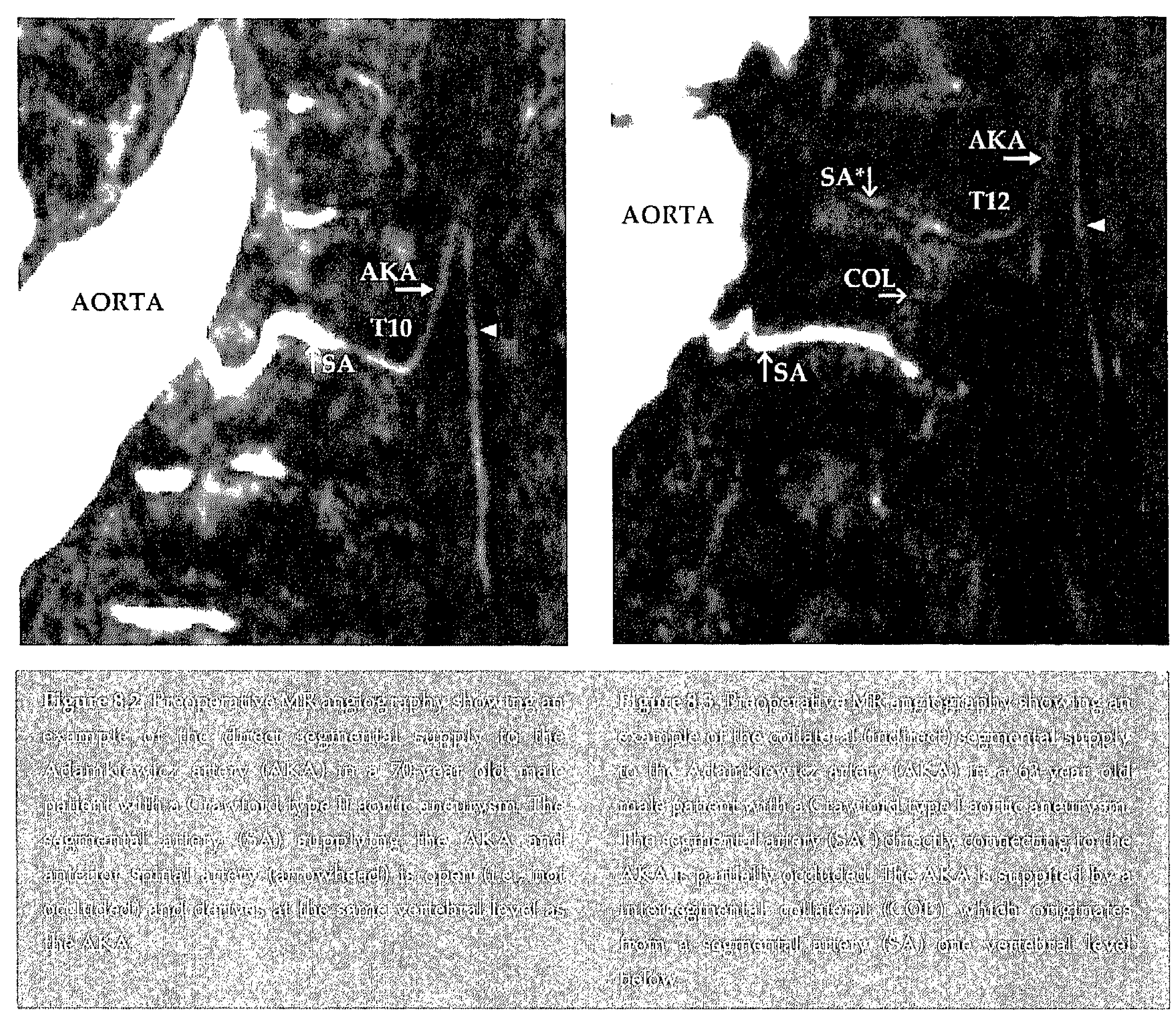

the AKA was occluded at its origin in the aortic wall in $40 \%(24 / 60)$ of the cases. In these cases, the (partially) occluded SA and, thereby, the AKA were supplied indirectly through a intersegmental collateral (example in Figure 8.3). In six patients, two vertebral levels separated the open (i.e., direct connection with the aorta) SA and the partially occluded segmental supplier of the AKA. In the 18 remaining patients, there was only 1 vertebral level difference. The AKA arose in all cases
$(100 \%)$ between the vertebral levels T8 and L2 and was found to derive in $72 \%(43 / 60)$ from the left side and in $28 \%(17 / 60)$ from the right side. A summary of AKA and GARV localizations is provided in Figure 8.4.

The number of open SAs (i.e., direct connection with the aorta) from $\mathrm{T} 3$ down to $\mathrm{L} 5$ in the atherosclerotic group (9.4 \pm 5.0 ; mean \pm SEM) was significantly lower $\left(P<3 \times 10^{-6}\right)$ compared with the dissection group (18.2 \pm 5.6). Furthermore, it was found that in the 


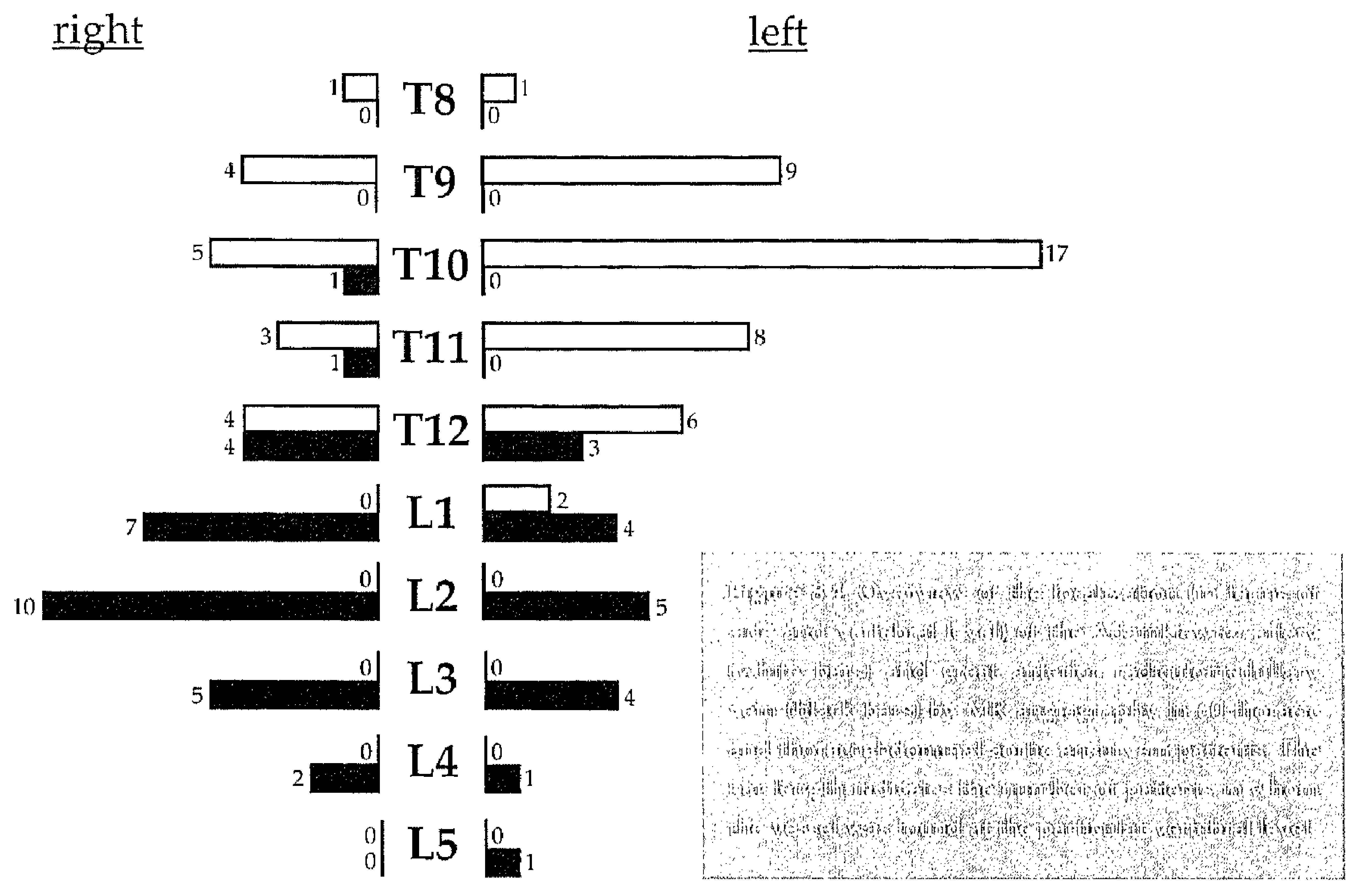

atherosclerotic group, the AKA was significantly $(P<0.005)$ more often supplied through an intersegmental connection compared with the dissection group, in which the supply to the AKA was mostly direct from the aorta and SA (Table 8.1).

\section{$M E P$ registration}

Adequate MEP monitoring could be achieved in $100 \%$ of the patients. The SA either directly or indirectly (i.e., by an intersegmental collateral) supplying the AKA was cross-clamped in $44(73 \%)$ of the 60 patients (Figure 8.5). Excluding the segmental supplier of the AKA from the direct (aortic) circulation led in 32\% (14/44) of cases to MEP decline (Figure 8.5). When the SA supplying the AKA was outside the cross-clamped area (16 cases), the MEPs remained stable (Figure 8.5). Reattachment of the preoperatively localized SA supplying the AKA either directly or indirectly restored the MEPs in 12 $(86 \%)$ of the 14 patients.

Combining $M R$ angiography with MEPs

A significant association $(P<0.01)$ was found between aortic cross-clamping of the SA supplying the AKA and MEP decline (Figure 8.5). This means that the relative position of the SA supplying the AKA to the cross-clamped aorta area matters to the intraoperative spinal cord function. However, the positive predictive value for crossclamping the SA supplying the AKA and MEP 
Table 8.1. Two-by-two table showing the number of patients for the atherosclerosis and dissection group in whom the Adamkiewicz artery was directly or indirectly (intersegmental connection) supplied.

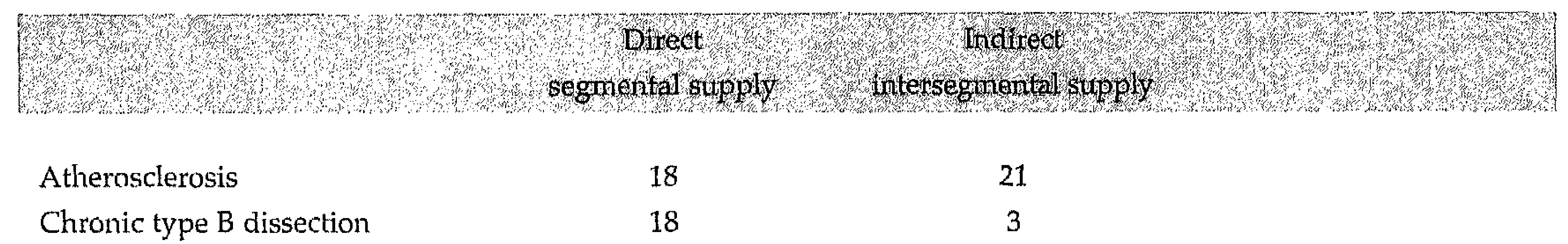

The Fischer statistical exactness test revealed that the presence of indirect intersegmental blood supply was statistically more prevalent $(P<0.005)$ in atherosclerosis $(54 \%)$ than in dissection $(14 \%)$ patients.

decline was only $32 \% \quad(95 \%$ confidence interval, 20-45\%), whereas the negative predictive value was $100 \%$ (95\% confidence interval, 83-100\%).

The table shows that the relative number of patients in the atherosclerosis group for whom intersegmental collateral connections were observed was higher than for the dissection group $(P<0.005)$. This means that in patients with atherosclerosis, the spinal cord blood supply depends more strongly on collaterals than it does in patients with an aortic dissection.

\section{Clinical outcome}

No intraoperative death or renal failure occurred in this series of patients. After surgery, eight patients $(13 \%)$ died in the hospital: one patient with a TAA (respiratory failure), four patients with a type II TAAA (cardiac and respiratory failure), two patients with a type III TAAA (sepsis and respiratory failure), and one patient with a type IV TAAA (sepsis).

In two patients (one type II TAAA and one type III TAAA), acute paraplegia became evident after the procedure. This neurologic deficit was anticipated because MEPs were entirely absent at the end of the procedure. In these two patients, sequential clamping of the thoracic and abdominal aorta was associated with a gradual decrease of MEPs to nonmeasurable amplitudes. Despite increasing mean arterial and distal aortic pressures and revascularization of all available SAs, including the one supplying the AKA, MEP signals did not return.

In one type II TAAA patient, delayed paraplegia occurred. In this patient, exclusion of the entire aorta did not lead to a decline in MEPs. Because of the very poor quality of the aorta and because no MEP declines were observed during the entire operation, no attempts were undertaken to revascularize any of the encountered back-bleeding SAs, including the supplier of the AKA. At the intensive care unit, the patient experienced a short period of hypotension. Thereafter, he was unable to move his legs.

\section{DISCUSSION}

In this study, we showed that MR angiography is able to consistently (100\%) visualize the blood-supplying trajectories to the spinal cord, including the aorta, SAs, AKA, and ASA. Moreover, the venous outlet trajectory, which 
Inclusion

Cross-clamping segmental supplier Adamkiewicz artery
Decline of motor evoked potentials

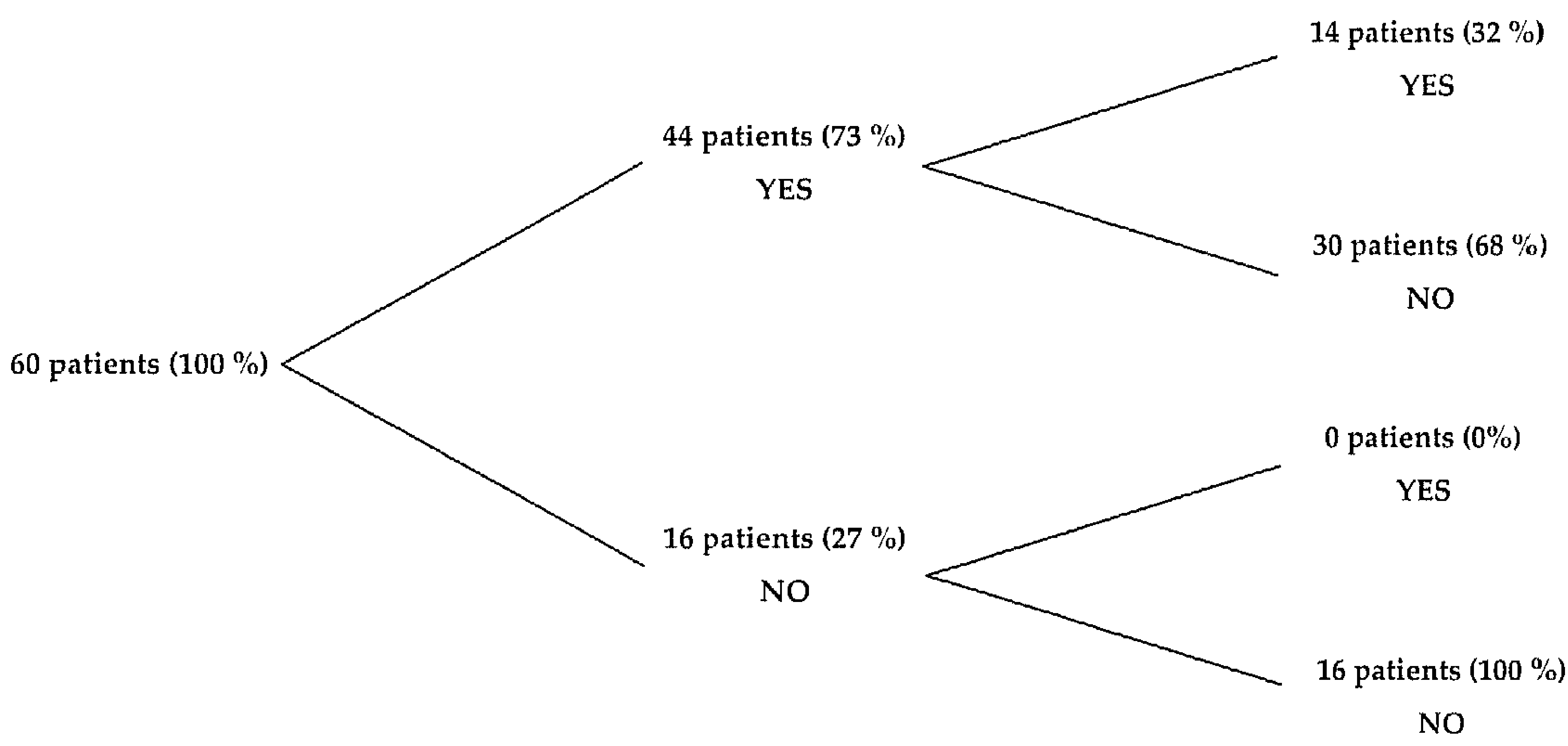

has a similar spatial configuration and therefore may confound the detection of the $\mathrm{AKA}$, can be differentiated from the required arterial inflow trajectories to the spinal cord by using dynamic two-phase MR angiography. By combining MR angiography with intraoperative MEP monitoring, it was demonstrated that the location of the SA supplying the AKA relative to the aortic cross-clamp area is significantly associated with intraoperative spinal cord function.

Previously, the existence and the importance of preoperative localization of the AKA and its supplying SA were doubted. One reason for this was that the AKA could not be identified in all TAA(A) patients by using catheter angiography 4,11 . However, in our opinion and those of others ${ }^{11}$, the inability to localize the AKA should not lead to the conclusion that the AKA does not exist. Detection failure of catheter angiography might be due to calcification of the aortic orifices of the SAs in cases with atherosclerosis or might be caused by a disrupted or detached intima layer in patients with an aortic dissection. With MR angiography, we were 
able to localize the AKA in $100 \%$ of cases between T8 and L2, which concurs with the anatomic localizations found in postmortem studies 23,24. Another argument provided against preoperative angiography is that paraplegia still occurred in some cases in which the AKA was identified and its supplying SA was reattached ${ }^{11}$. Moreover, Griepp et $\mathrm{al}^{8}$ noticed in their group of patients that when all SAs were oversewn (i.e., not revascularized), no paraplegia was observed. These findings gave rise to the question of whether reattachment of the SA connecting to the AKA is essential to maintain spinal cord function.

Conversely, studies have been published that emphasize that revascularization of SA is truly essential ${ }^{6}$ or at least beneficial ${ }^{7}$ to prevent paraplegia. To assess whether a specific SA requires revascularization, the surgeon needs information on the contribution to the spinal cord function of the SA considered. To gain insight into SA contribution, MEP monitoring has been used. MEP monitoring has been shown to be a reliable and reproducible method to decrease the number of patients with postoperative paraplegia $5,12,13,22$. Theoretically, the most optimal use of MEPs would be to cross-clamp each pair of SAs individually and assess its pairwise contribution. However, because this would tremendously increase the total crossclamp and operation time, which is associated with a higher chance for developing paraplegia and other complications, this approach is not applied. To exclude a minimal number of SAs, in this study segmental cross-clamping of the aorta was applied. In this way, approximately three to four pairs of SAs were excluded at once from the direct aortic circulation.
By using the segmental cross-clamping approach in combination with the MEP technique and distal aortic perfusion, a significant association was found for the location of the SA supplying the AKA relative to the aortic cross-clamp area. When the segmental supplier of the AKA was outside the cross-clamped area, no changes in the MEPs were observed (i.e., 100\% negative predictive value). This finding can be of use in determining preoperative surgical strategies, for instance, to define the aortic clamp positions or the distal landing zone of an endovascular stent graft. Also, during open aneurysm repair, knowledge of the localization of the SA supplying the AKA can be of importance. This especially accounts for situations in which a segment of the aorta is opened and multiple back-bleeding SAs are encountered and the MEPs decline. Selective, fast, and adequate revascularization of the crucial SA is mandatory in such a situation, which now can be pinpointed by preoperative MR angiography. Considering the limited time available, starting with reattachment of non-critical SAs can lead to paraplegia. This could also be the reason why in studies using MEPs in combination with distal aortic perfusion and CSF drainage, but without the preoperative knowledge of the SA supplying the AKA, paraplegia could not be prevented in all cases ${ }^{12}$.

In our series, using distal aortic perfusion, 14 cases of MEP decline were observed in 44 cases in which the SA supplying the AKA was cross-clamped. This shows that there is not a strong predictive relation between the exclusion of the SA supplying the AKA from the direct aortic circulation and MEP decline, because in most patients $(68 \%)$ no MEP declines were observed. This also implies that 
the segmental supplier of the AKA was not crucial in these cases to maintain spinal cord function. Spinal cord function was probably preserved by a combination of intraoperative protective strategies (distal aortic perfusion, cooling, and CSF drainage). However, although these protective strategies may be sufficient to maintain the spinal cord function during surgery, another mechanism, such as collateral blood supply, must exist to preserve the blood supply to the spinal cord after surgery. In this study, at least a part of such a collateral supply to the spinal cord was visualized, because in 24 patients the spinal cord blood supply through the AKA was dependent on intersegmental collateral connections. As one might expect, this was observed more often in the patient group with atherosclerosis compared with the dissection group and likely relates to the fact that the atherosclerotic group had less numerous open SAs compared with the dissection group. In patients with atherosclerosis, the chronic but slow process of plaque formation may provide sufficient time for collaterals to form and mature to realize sufficient collateral circulation, which is likely stimulated by the increased shear stress in the collateral trajectories in response to the occlusion of many SAs. For patients with aortic dissections, on the contrary, the pathologic process is more acute, the required time to form collaterals is consequently less, and the increase in shear stress is less likely as multiple SAs remain open.

According to our experience ${ }^{5}$ and the literature ${ }^{8}$, it is known that collaterals can play an important role in the blood supply to the spinal cord and may even in some cases be sufficient to maintain spinal cord function ${ }^{8}$. In consideration of the foregoing, it would be relevant to develop MR acquisition and postprocessing techniques to actually visualize with ultra-highspatial resolution the trajectories of the collateral networks supplying the AKA. Although a collateral network might be sufficient to maintain spinal cord function, this provides in our opinion no justification to oversew each SA. This would make the spinal cord blood supply dependent on only the collateral circulation and make it potentially vulnerable to low-flow conditions and low blood pressure ${ }^{11,25}$. To emphasize that this can be quite hazardous, the postoperative paraplegic case is recalled, in which the spinal functionality was lost because of low blood pressure. Therefore, it is strongly advised to revascularize the segmental supplier of the AKA even if MEPs show no decline.

In conclusion, preoperative MR angiography combined with MEP neuromonitoring enables determination of the arterial inlet trajectory to the spinal cord that is sufficient to maintain spinal cord function. This arterial trajectory to the AKA may either be direct or through a collateral network and can now be visualized by MR angiography. In this study, it was shown that when the segmental supplier of the AKA was not cross-clamped, spinal cord integrity was preserved (i.e., the negative predictive value was $100 \%$ ). This may help to plan cross-clamp strategies in a attempt to further decrease the incidence of postoperative spinal cord complications in the near future. Furthermore, it can be of additional value in cases in which endovascular treatment attempts to cover the entire descending thoracic aorta, including the SA supplying the AKA. In these cases, caution for spinal cord ischemia is justified, and protective measures are warranted. 


\section{REFERENCES}

1. Thron A. Anatomy of the spinal cord's blood supply. In: Thron A, ed. Vascular anatomy of the spinal cord. Wien: Springer-Verlag; 1988:8-12.

2. Lasjaunias P. Spinal and spinal cord arteries and veins. In: Lasjaunias P, Berenstein, A, Ter Brugge K.G., ed. Surgical Neuroungiography. Berlin: Springer-Verlag; 2001:73-160.

3. Yoshioka K, Niinuma H, Kawazoe K, Ehara S. Threedimensional demonstration of the Adamkiewicz artery and its collateral supply with contrastenhanced magnetic resonance angiography. Eur I Cardiothorac Surg 2004;26:440-1.

4. Kieffer E, Fukui S, Chiras J, Koskas F, Bahnini A, Cormier E. Spinal cord arteriography: a safe adjunct before descending thoracic or thoracoabdominal aortic aneurysmectomy. J Vasc Surg 2002;35:262-8.

5. Jacobs MJ, de Mol BA, Elenbaas T, et al. Spinal cord blood supply in patients with thoracoabdominal aortic aneurysms. J Vasc Surg 2002;35:30-7.

6. Safi HJ, Bartoli S, Hess KR, et al. Neurologic deficit in patients at high risk with thoracoabdominal aortic aneurysms: the role of cerebral spinal fluid drainage and distal aortic perfusion. J Vasc Surg 1994;20:434-44; discussion 42-3.

7. Hyodoh $\mathrm{H}$, Kawaharada $\mathrm{N}$, Akiba $\mathrm{H}$, et al. Usefulness of preoperative detection of artery of Adamkiewicz with dynamic contrast-enhanced MR angiography. Radiology 2005;236:1004-9.

8. Griepp RB, Ergin MA, Galla JD, et al. Looking for the artery of Adamkiewicz: a quest to minimize paraplegia after operations for aneurysms of the descending thoracic and thoracoabdominal aorta. / Thorac Cardiovasc Surg 1996;112:1202-13; discussion 13-5.

9. Biglioli P, Spirito R, Porqueddu M, et al. Quick, simple clamping technique in descending thoracic aortic aneurysm repair. Ann Thorac Surg 1999;67:1038-43; discussion 43-4.
10. Acher CW, Heisey DM. Regarding "Importance of intercostal artery reattachment during thoracoabdominal aortic aneurysm repair". I Vasc Surg 1998;28:570-1.

11. Williams GM, Roseborough GS, Webb TH, Perler BA, Krosnick T. Preoperative selective intercostal angiography in patients undergoing thoracoabdominal aneurysm repair. / Vasc Surg 2004;39:314-21.

12. Jacobs MJ, Mess W, Mochtar B, Nijenhuis RJ, Statius van Eps RG, Schurink GW. The value of motor evoked potentials in reducing paraplegia during thoracoabdominal aneurysm repair. I Vasc Surg 2006;43:239-46.

13. Jacobs MJ, Elenbaas TW, Schurink GW, Mess WH, Mochtar B. Assessment of spinal cord integrity during thoracoabdominal aortic aneurysm repair. Ann Thorac Surg 2002;74:S1864-6; discussion S92-8.

14. Nijenhuis RJ, Leiner T, Cornips EM, et al. Spinal cord feeding arteries at MR angiography for thoracoscopic spinal surgery: feasibility study and implications for surgical approach. Radiology 2004;233:541-7.

15. Nijenhuis $R J$, Gerretsen $S$, Leiner $T$, Jacobs $M J$, van Engelshoven JM, Backes WH. Comparison of 0.5-M Gd-DTPA with 1.0-M gadobutrol for magnetic resonance angiography of the supplying arteries of the spinal cord in thoracoabdominal aortic aneurysm patients. J Magn Reson Imaging 2005;22:136-44.

16. Kawaharada N, Morishita K, Hyodoh H, et al. Magnetic resonance angiographic localization of the artery of Adamkiewicz for spinal cord blood supply. Am Thorac Sury 2004;78:846-51; discussion 51-2.

17. Takase K, Sawamura Y, Igarashi K, et al. Demonstration of the artery of Adamkiewicz at multidetector row helical CT. Radiology 2002;223:39-45.

18. Kudo $K$, Terae $S$, Asano $T$, et al. Anterior spinal artery and artery of Adamkiewicz detected by using multidetector row CT. AJNR Am J Neuroratiol 2003;24:13-7. 
19. Yamada N, Okita Y, Minatoya $K$, et al. Preoperative demonstration of the Adamkiewic 2 artery by magnetic resonance angiography in patients with descending or thoracoabdominal aortic aneurysms. Eur J Cardiothorac Surg 2000;18:104-11.

20. Minatoya K, Karck M, Hagl C, et al. The impact of spinal angiography on the neurological outcome after surgery on the descending thoracic and thoracoabdominal aorta. Ann Thorac Surg 2002;74:S1870-2; discussion S92-8.

21. Kawaharada N, Morishita K, Fukada J, et al. Thoracoabdominal or descending aortic aneurysm repair after preoperative demonstration of the Adamkiewicz artery by magnetic resonance angiography. Eur / Cardiothorac Surg 2002;21:970-4.

22. Jacobs MJ, Mess WH. The role of evoked potential monitoring in operative management of type I and type II thoracoabdominal aortic aneurysms. Semin Thorac Cardiounsc Surg 2003;15:353-64.

23. Koshino T, Murakami G, Morishita $K$, Mawatari $T$, Abe T. Does the Adamkiewicz artery originate from the larger segmental arteries? I Thorac Cardiounsc Surg 1999;117:898-905.

24. Morishita K, Murakami G, Fujisawa $Y$, et al. Anatomical study of blood supply to the spinal cord. Ann Thorac Surg 2003;76:1967-71.

25. Chiesa R, Melissano G, Marrocco-Trischitta MM, Civilini $E$, Setacci F. Spinal cord ischemia after elective stent-graft repair of the thoracic aorta. I Vasc Surg 2005;42:11-7. 


\section{Value and limitations of MR angiography in spinal arteriovenous malformations and dural arteriovenous fistulas}




\section{ABSTRACT}

Purpose: To study the validity of MR angiography for identification of spinal arteriovenous (AV) abnormalities.

Materials and Methods: 34 consecutive patients with suspicion of spinal vascular abnormalities underwent intra-arterial catheter (X-ray) angiography after MR angiography. The level and side of the suspected spinal dural arteriovenous fistula (SDAVF) and the feeding arteries in spinal arteriovenous malformations (SAVMs) were determined from the MR angiography and compared to catheter angiography.

Results: Catheter angiography revealed DAVF in 20 of which 19 were spinal and 1 was tentorial with spinal drainage and SAVM in 11 patients. In 3 patients MR angiography and catheter angiography were both normal. For detection of spinal arteriovenous abnormalities neither false-positive nor false-negative MR angiography results were obtained. The MR angiography derived level of the feeding artery in SDAVF agreed with catheter angiography in 14 out of 19 cases. In 5 cases a mismatch of one vertebral level (not side) was noted for the feeding artery. For the tentorial AVF only the spinal drainage was depicted, the feeding artery was outside the MR angiography field of view. In intradural SAVM the main feeding artery was identified by MR angiography in 10 of 11 patients. MR angiography could differentiate between glomerular and fistulous SAVM in 4 out of 6 cases and between sacral SDAVF and filum terminale SAVM in 2 of 5 cases.

Conclusions: MR angiography reliably detects or excludes various types of spinal AV abnormalities and localizes the (predominant) arterial feeder of most spinal AV shunts.
Although classification the subtype of SAVMs remains difficult with MR angiography it greatly helps to focus subsequent catheter angiography. 


\section{INTRODUCTION}

Arteriovenous (AV) shunts of the spinal cord and its meninges (i.e. spinal arteriovenous malformations (SAVM) and spinal dural arteriovenous fistulae (SDAVF) are rare and still under-diagnosed entities. If not treated properly $\mathrm{AV}$ shunts can lead to considerable morbidity with progressive spinal cord symptoms and myelopathy due to venous congestion. Usually MRI is sufficient to raise the suspicion of a spinal vascular malformation. However, spinal cord intraarterial catheter (X-ray) angiography, also known as digital subtraction angiography, comprising selective arterial catheterization is still regarded as the only technique sufficiently reliable for the pre-treatment, detection and characterization of such vascular malformations ${ }^{1-3}$. Despite the superior image quality of catheter angiography there are a number of drawbacks using this imaging technique. Even in experienced hands, selective catheterization of arteries feeding the spinal cord carries the risk of neurologic complications ${ }^{4}$. The most important drawback is related to the fact that the location(s) of the artery(ies) supplying the AV shunt is (are) not known prior to angiography and there are no MR-based predictors (such as location and extent of the cord oedema) of the level of the shunt ${ }^{5}$. Therefore, to localize the possible feeder(s), selective catheterization of all arteries potentially supplying the spinal cord and its meninges may be required. These include both vertebral, costocervical and thyrocervical trunks, all intercostal lumbar and iliolumbar arteries $^{5}$. This can lead to often lengthy and sometimes multiple catheterization procedures with high radiation exposures in which large volumes of potentially nephrotoxic contrast agents are administered ${ }^{6}$.

For these reasons spinal cord MR angiography ${ }^{7}$ is evolving as a potentially attractive tool for the detection and evaluation of spinal cord vascular pathology prior to the catheter angiography examination. In the last decade several MR angiography techniques have been developed. First, blood flow dependent techniques such as phase contrast angiography $(\mathrm{PCA})^{8}$ and three-dimensional contrast-enhanced time of flight imaging ( $3 \mathrm{D}$ CE-TOF) ${ }^{9}$ were used. These techniques could confirm the presence of abnormally dilated spinal cord arteries or abnormal intraspinal veins, respectively. However, they failed to localize the site of shunting in SDAVF, due to the limited temporal resolution and long acquisition time. Binkert et $\mathrm{al}^{10}$ were the first who realized a fast (24 seconds) MR angiography technique that was able to depict the feeding artery to the SDAVF or SAVM. Although the necessity for a fast $M R$ angiography technique was recognized $6,10,11$, it was not yet possible to depict normal intradural arteries, which is mandatory to detect and preserve the specific blood supply to the spinal cord in case of treatment. In addition, detection of feeders of AV shunts in a single MR angiography session was still limited by the small size of the craniocaudal field of view ${ }^{11}$. As feeding arteries to spinal vascular shunts may arise from all arteries potentially supplying the spinal cord, a technique with the largest possible field of view seems therefore necessary to screen for any kind of vascular malformation. Besides detection, it is also important to characterize the subtypes of spinal AV abnormalities as they are subject to different therapeutic approaches 5 . 
In the present study patients with a suspected spinal AV abnormality were examined with MR angiography featuring a large $(50 \mathrm{~cm})$ craniocaudal field of view and centric k-space sampling, which enabled the visualization of both normal and pathologic arteries and veins. The MR angiography images were compared to catheter angiography with regard to the following features: (i) detection of the presence of a spinal AV shunt, (ii) localization of its arterial feeder(s), and (iii) characterization of the type of $\mathrm{AV}$ malformation.

\section{MATERIALS and METHODS}

\section{Patients}

Between December 2003 and April 2006, 34 consecutive patients ( 31 males and 3 females) with suspected spinal AV shunts were investigated in two centers (Aachen University Hospital, 28 patients; Maastricht University Hospital, 6 patients). The mean age was 59 years (range, 29-78 years). Referral to MR angiography was based on clinical data and previous MRI findings raising the suspicion of a spinal AV malformation (i.e. cord oedema and/or perimedullary enlarged vessels). In addition to conventional structural MRI of the entire spinal cord, all patients underwent a single MR angiography examination as described below during their routine diagnostic work-up prior to catheter angiography The interval between MR angiography and first catheter angiography examination ranged from 1 to 4 days.

\section{$M R$ angiography}

Imaging was performed on a clinical 1.5Tesla MRI system equipped with a phased- array spine coil. Patients were imaged in the supine position. The MRI protocol comprised three different pulse sequences ${ }^{12}$. Typically, the MRI exam took 30 minutes to perform. First, T2-weighted survey images were acquired to depict the course of the spinal cord as an anatomic reference. Survey scans were acquired in axial and sagittal orientations. Pulse sequence parameters were TR 2686 msec; TE $120 \mathrm{msec}$; flip angle $90^{\circ}$; field of view (FOV) $500 \mathrm{~mm}$, matrix size $320 \times 480$ (phase encoding $x$ frequency encoding), twelve 4-mm thick contiguous slices.

Second, MR fluoroscopy was used to determine the optimal scan delay between contrast injection and the start of the MR angiography acquisition. For this purpose a 2 $\mathrm{mL}$ test bolus of gadolinium based contrast agent (gadopentetate dimeglumine (GdDTPA), vial concentration $0.5 \mathrm{~mol} / \mathrm{L}$ ) was administered. The test bolus was administered at the same injection rate $(3 \mathrm{~mL} / \mathrm{sec})$ as the final bolus utilizing a power-injector, followed by a $25 \mathrm{~mL}$ saline flush. The MR fluoroscopy sequence consisted of a single $80-\mathrm{mm}$ thick slice, which was sagittally positioned through the aorta. This slice was acquired once each second for two minutes. The pulse sequence parameters were TR 5.2 msec, TE $1.4 \mathrm{msec}$, FA $35^{\circ}$, FOV $450 \mathrm{~mm}$, and matrix size $205 \times 256$. Viewing the series of acquired bolus timing images, the scan delay was defined as the time between start of test bolus injection and maximal enhancement of the entire aorta down to the bifurcation. The obtained scan delay was used to synchronize the sampling of the center of k-space with the peak contrast agent concentration in the subsequent MR angiography acquisition.

To facilitate subtraction MR angiography, four consequent pre-contrast phases were 


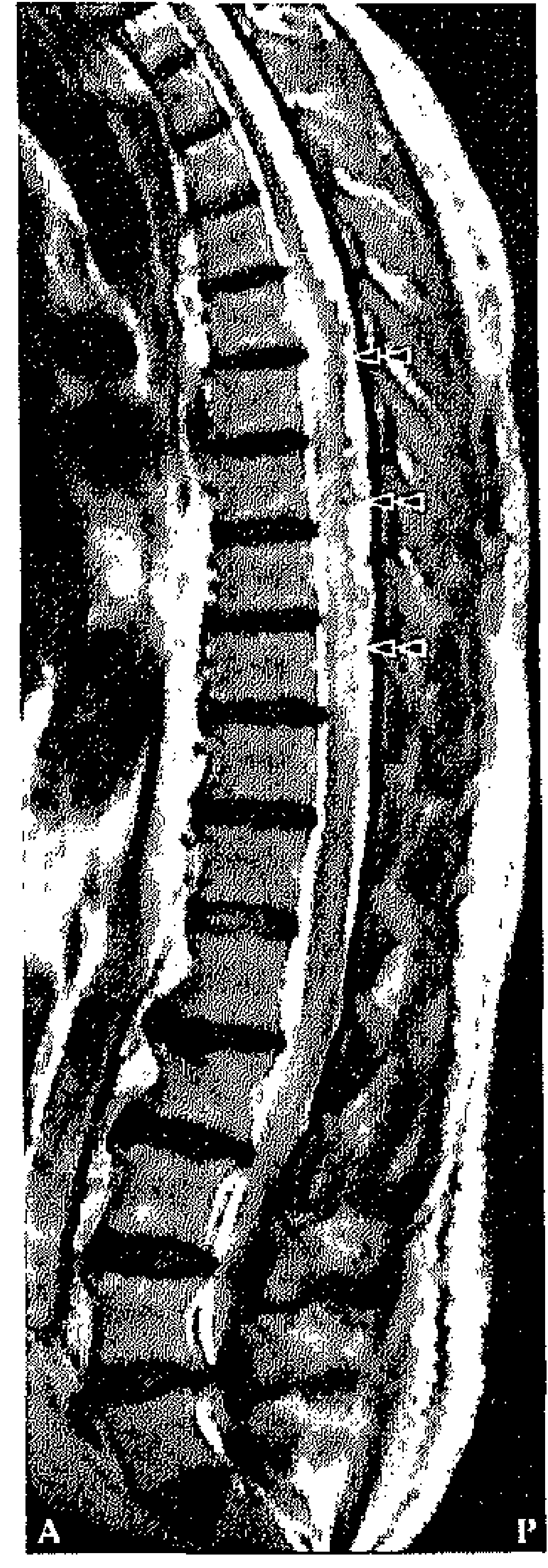

a

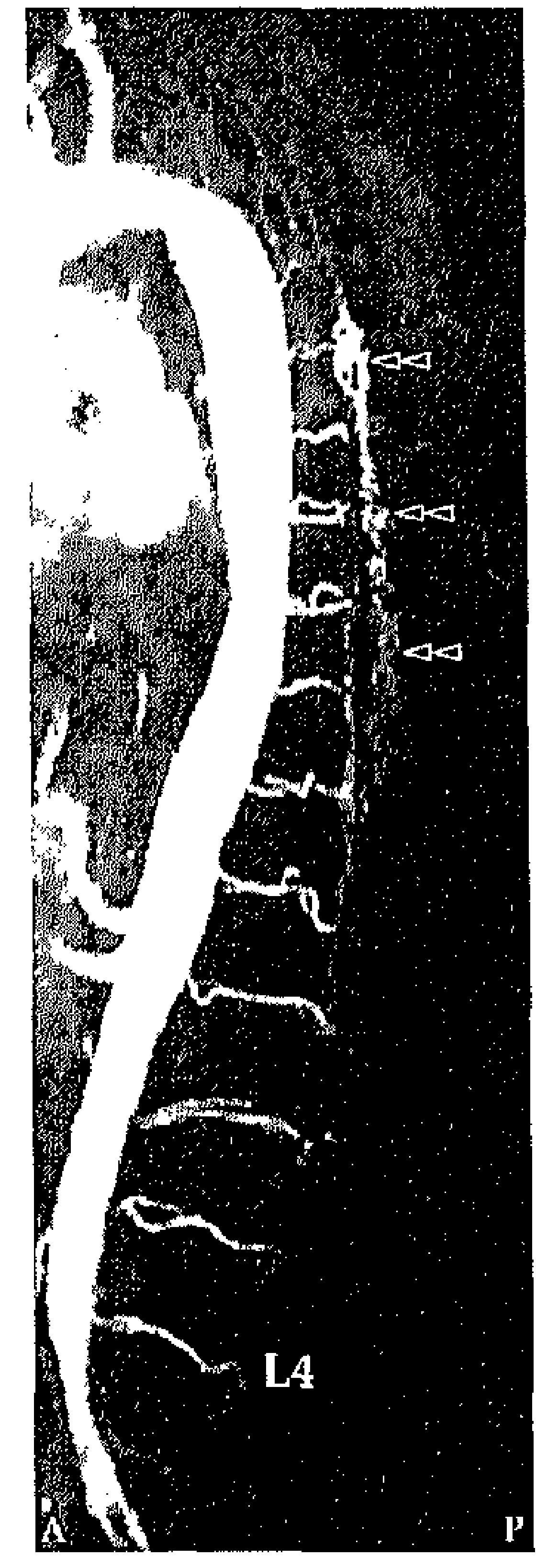

b

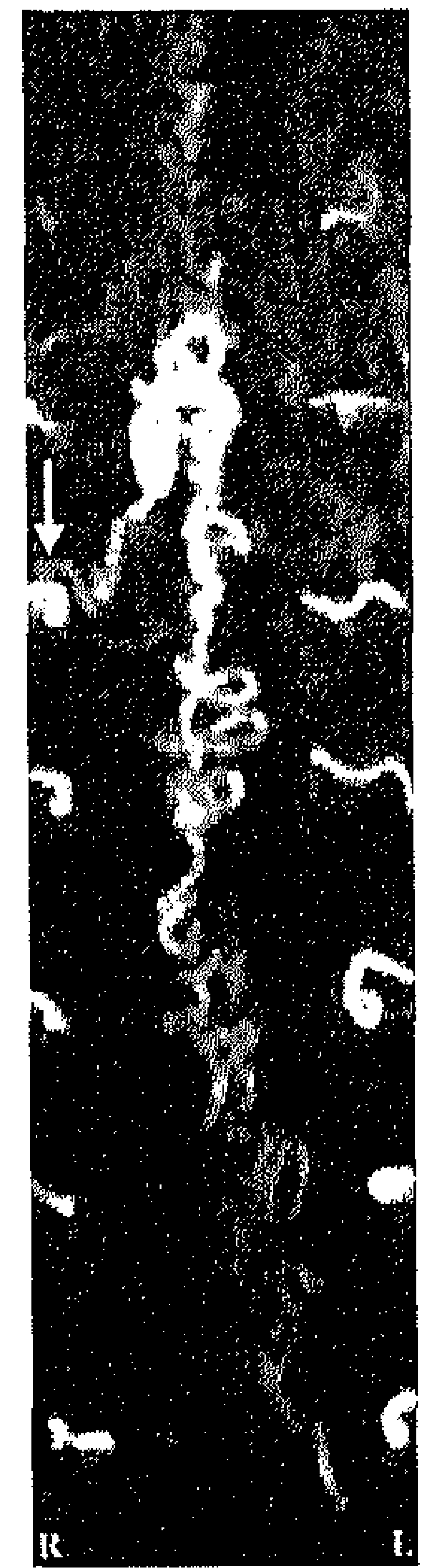

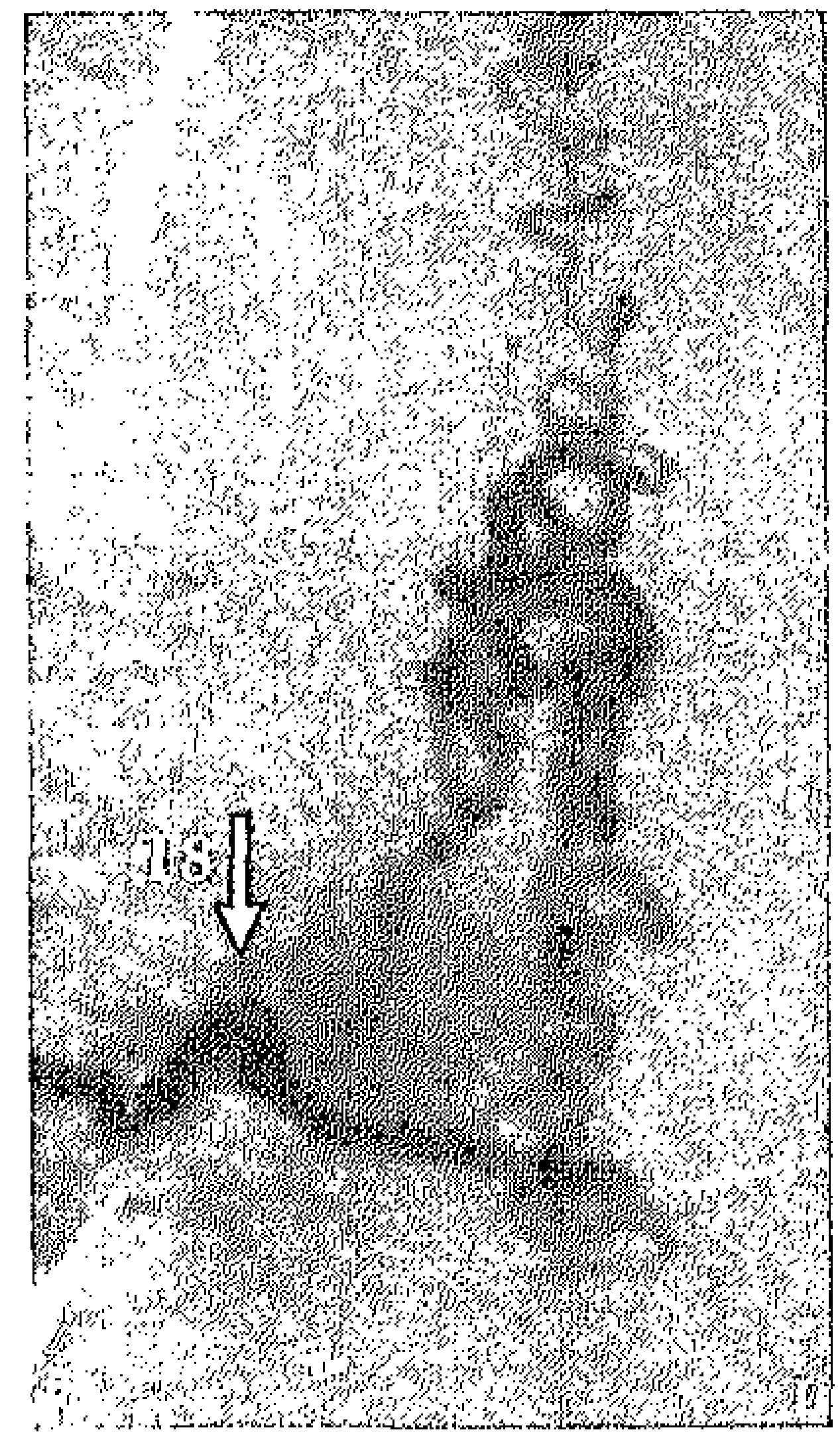

d

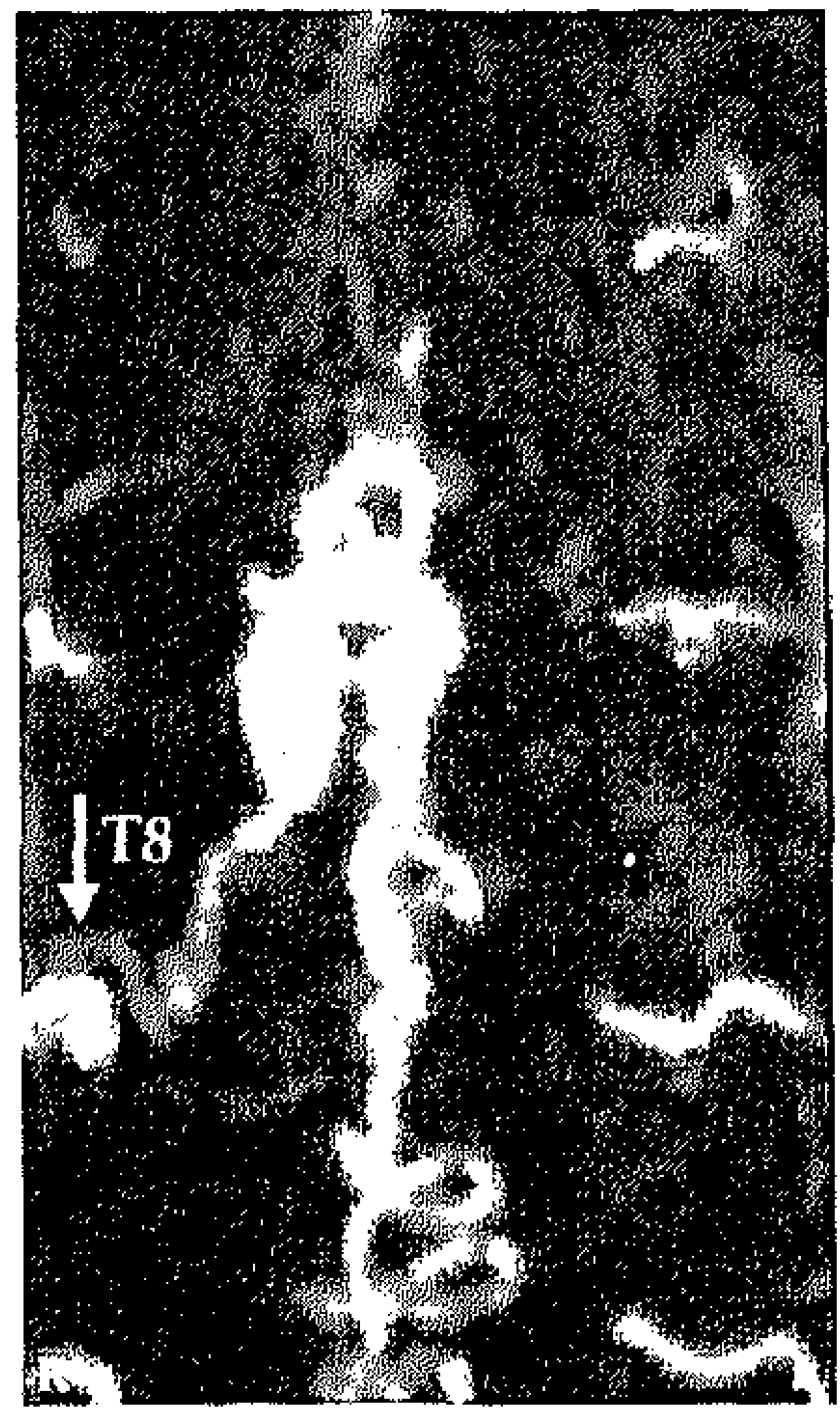

e

C

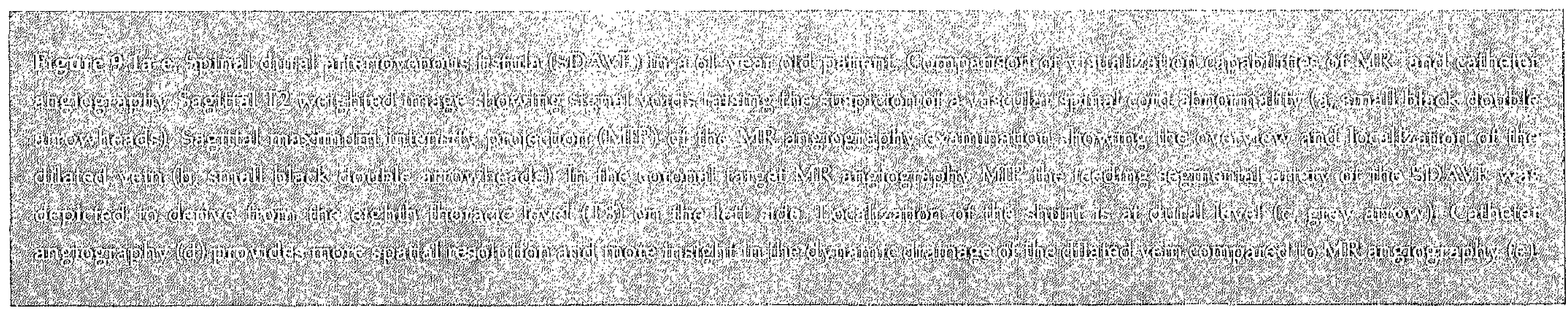

acquired comprising exactly the same pulse sequence parameters as the MR angiography sequence. These pre-contrast scans were (automatically) averaged to reduce noise propagation, which is inherent to the subtraction of pre-contrast from images, in order to enhance small vessel conspicuity. Finally, a dynamic two-phase threedimensional spoiled fast gradient echo pulse sequence with $45 \mathrm{~mL}$ of contrast agent was utilized for MR angiography. The average precontrast images were subtracted from the corresponding images of the first phase. The two phases served to distinguish relatively early contrast enhancement, which mainly involves (normal and/or pathologic) arteries and pathologic (i.e., arterialized) veins, from later enhancement where arteries, arterialized veins but also normal veins are visualized together. Each MR angiography phase took 36 
Table 9.1. Dural Arteriovenous Fistula (DAVF) - Findings at MR and catheter angiography.

\begin{tabular}{|c|c|c|c|c|c|c|}
\hline Case No, & ex/ Age (y) & $\begin{array}{c}\text { Catheter } \\
\text { Mglograph } \\
\text { Level }\end{array}$ & side & 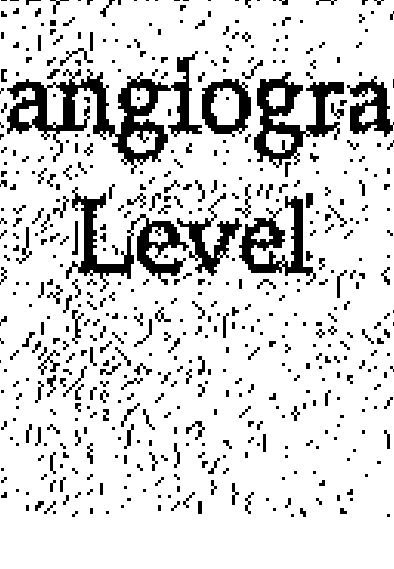 & tde & $\begin{array}{l}\text { Level and side } \\
\text { correct at } \\
\text { angiography }\end{array}$ \\
\hline 1 & $\mathrm{~m} / 69$ & L1 & $\mathrm{L}$ & L1 & L & Yes \\
\hline 2 & $\mathrm{~m} / 63$ & T6 & $\mathrm{L}$ & T6 & $L$ & Yes \\
\hline 3 & $\mathrm{f} / 75$ & T5 & $\mathrm{R}$ & T5 & $\mathrm{R}$ & Yes \\
\hline 4 & $\mathrm{~m} / 38$ & $\mathrm{~T} 7$ & $\mathrm{~L}$ & $\mathrm{~T} 7$ & $\mathrm{~L}$ & Yes \\
\hline 5 & $\mathrm{~m} / 64$ & $\mathrm{~S} 1 / 2$ & $\mathrm{R}$ & $\mathrm{S} 1 / 2$ & $\mathrm{R}$ & Yes \\
\hline 6 & $\mathrm{~m} / 61$ & T6 & $\mathrm{R}$ & T7 & $\mathrm{R}$ & No \\
\hline 7 & $\mathrm{~m} / 71$ & $\mathrm{~T} 6 / \mathrm{T} 7^{*}$ & $\mathrm{~L}$ & $\mathrm{~T} 6 / \mathrm{T} 7^{*}$ & $\mathrm{~L}$ & Yes \\
\hline 8 & $\mathrm{~m} / 72$ & T12 & $\mathrm{R}$ & T12 & $\mathrm{R}$ & Yes \\
\hline 9 & $\mathrm{~m} / 66$ & L3 & $\mathrm{L}$ & L3 & $\mathrm{L}$ & Yes \\
\hline 10 & $\mathrm{~m} / 72$ & L1 & $\mathrm{R}$ & L1 & $\mathrm{R}$ & Yes \\
\hline 11 & $\mathrm{~m} / 70$ & $\mathrm{~T} 5$ & $\mathrm{R}$ & T5 & $\mathrm{R}$ & Yes \\
\hline 12 & $\mathrm{~m} / 66$ & $\mathrm{~T} 6$ & $\mathrm{R}$ & T6 & $\mathrm{R}$ & Yes \\
\hline 13 & $\mathrm{~m} / 61$ & $\mathrm{~T} 8$ & $\mathrm{R}$ & $\mathrm{T} 8$ & $\mathrm{R}$ & Yes \\
\hline 14 & $\mathrm{~m} / 56$ & T5 & $\mathrm{R}$ & T6 & $\mathrm{R}$ & No \\
\hline 15 & $\mathrm{~m} / 55$ & $\mathrm{~T} 11$ & $\mathrm{R}$ & $\mathrm{T} 10$ & $\mathrm{R}$ & No \\
\hline 16 & $\mathrm{~m} / 66$ & T11 & $\mathrm{L}$ & $\mathrm{T} 10$ & $\mathrm{~L}$ & No \\
\hline 17 & $\mathrm{~m} / 50$ & $\mathrm{~T} 10$ & $\mathrm{~L}$ & $\mathrm{~T} 11$ & $\mathrm{~L}$ & No \\
\hline 18 & $\mathrm{~m} / 68$ & $\mathrm{~S} 1 / 2$ & L & $\mathrm{S} 1 / 2$ & $\mathrm{~L}$ & Yes \\
\hline $19^{\star \star}$ & $\mathrm{m} / 77$ & $\mathrm{~L} 2$ & $\mathrm{R}$ & $\mathrm{L} 2$ & $\mathrm{R}$ & Yes \\
\hline $20^{* * *}$ & $\mathrm{~m} / 63$ & \multicolumn{2}{|c|}{ Tentorial } & \multicolumn{2}{|c|}{ Outside FOV } & Not imaged \\
\hline
\end{tabular}

*The fistula was located at T7 left, supplied by the segmental arteries T6 and T7

**Epidural spinal AVF

***Tentorial DAVF. The fistula was located outside the field of view (FOV), but the venous drainage was clearly depicted with MR angiography

to 40 seconds, depending on the number of slices required to cover the entire axial crosssection of all vertebrae included. Imaging was performed without breath-hold. FOV was 500 $\mathrm{mm}$ in craniocaudal (frequency encoding) direction, matrix dimensions were $464 \times 512$ (phase encoding $x$ frequency encoding), and acquired pixel size was $0.9 \times 0.9 \mathrm{~mm}$. Acquired sagittal slices were $1.2 \mathrm{~mm}$ thick and interpolated (using zero-filling in k-space) during reconstruction to $0.6 \mathrm{~mm}$ thick slices.
The number of slices was individually adjusted (range 75-85; 45-51 $\mathrm{mm}$ ) to include the entire vertebral column usually from $\mathrm{T} 3$ down to S5 in the (craniocaudal) FOV. Contrast parameters of the spoiled fast gradient echo pulse sequence were TR 5.9 msec; TE 1.9 msec (partial echo 62.5\%); and flip angle $30^{\circ}$. To emphasize the arterial phase of the bolus enhancement relative to the venous enhancement, $k$-space was sampled using elliptical centric ordering in which the 


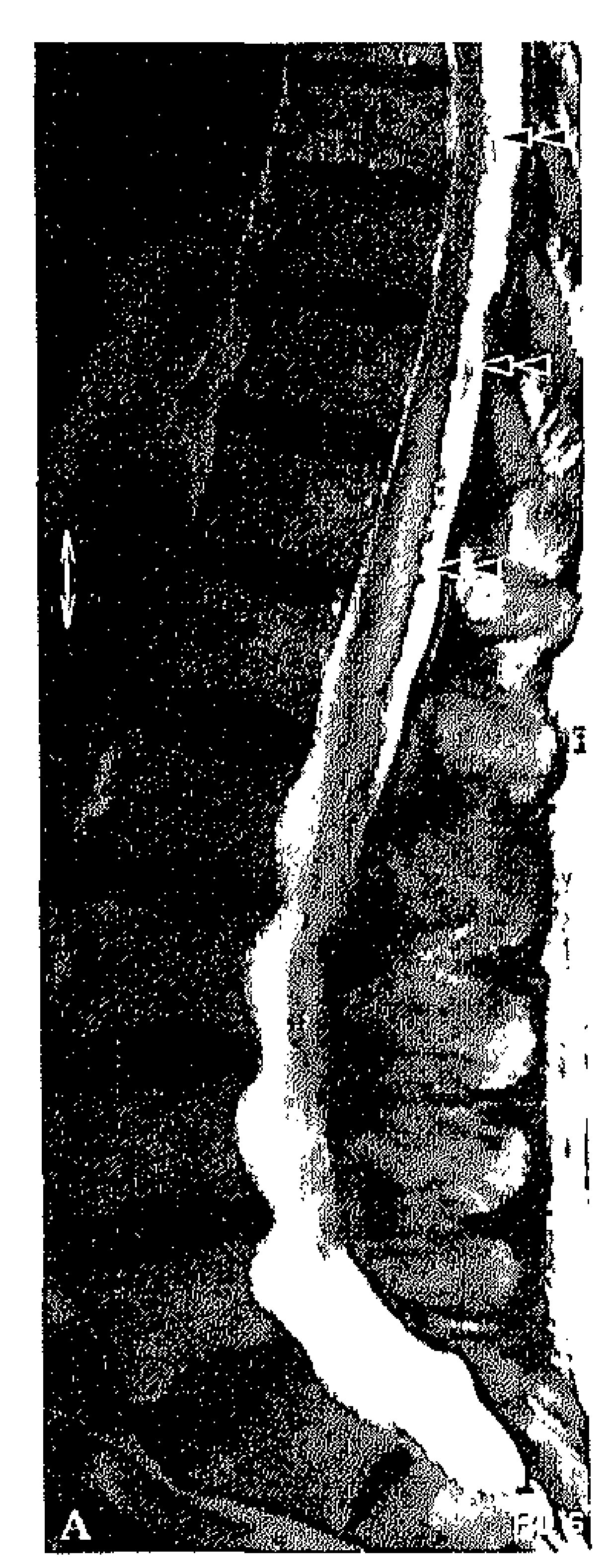

a
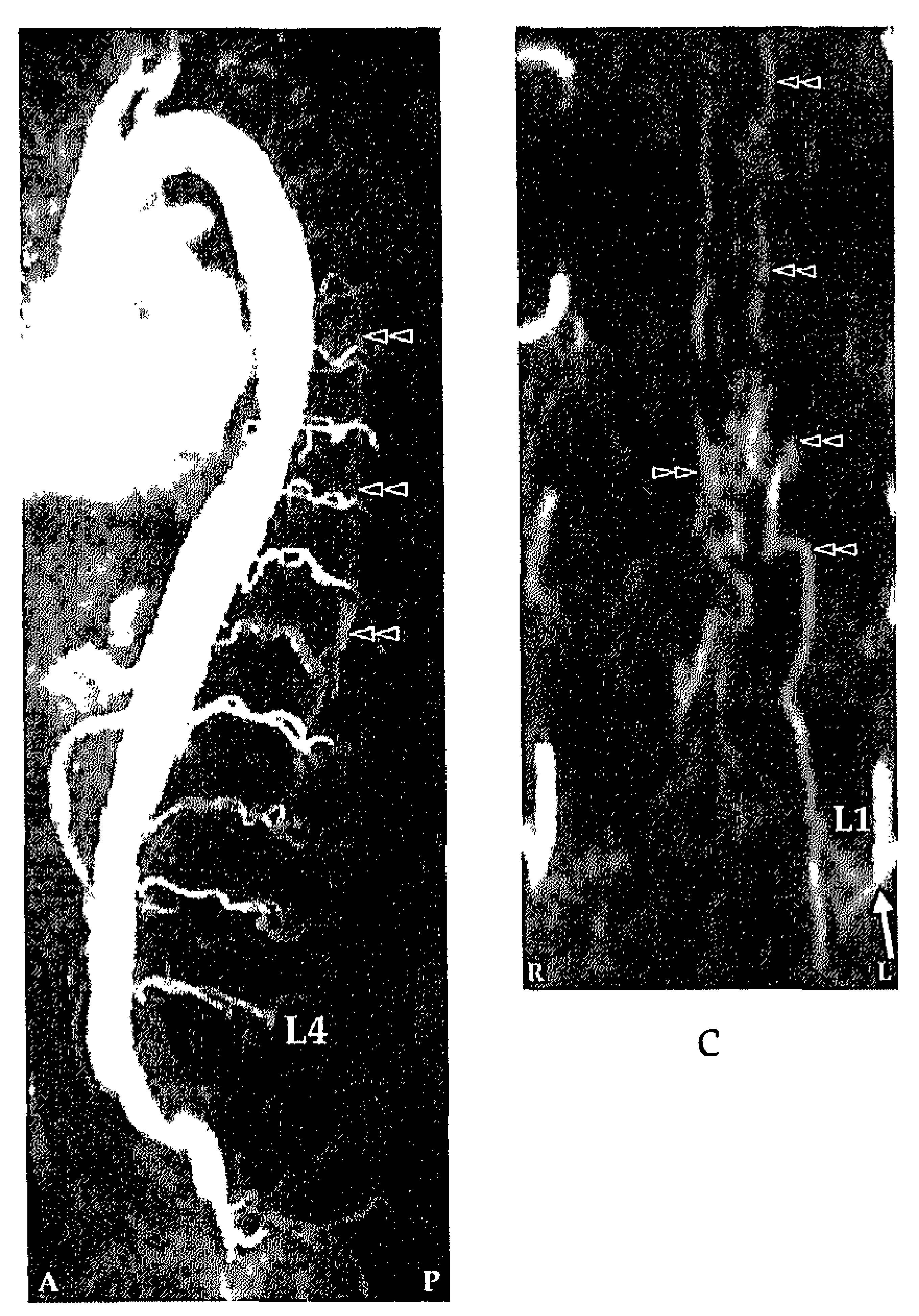

C

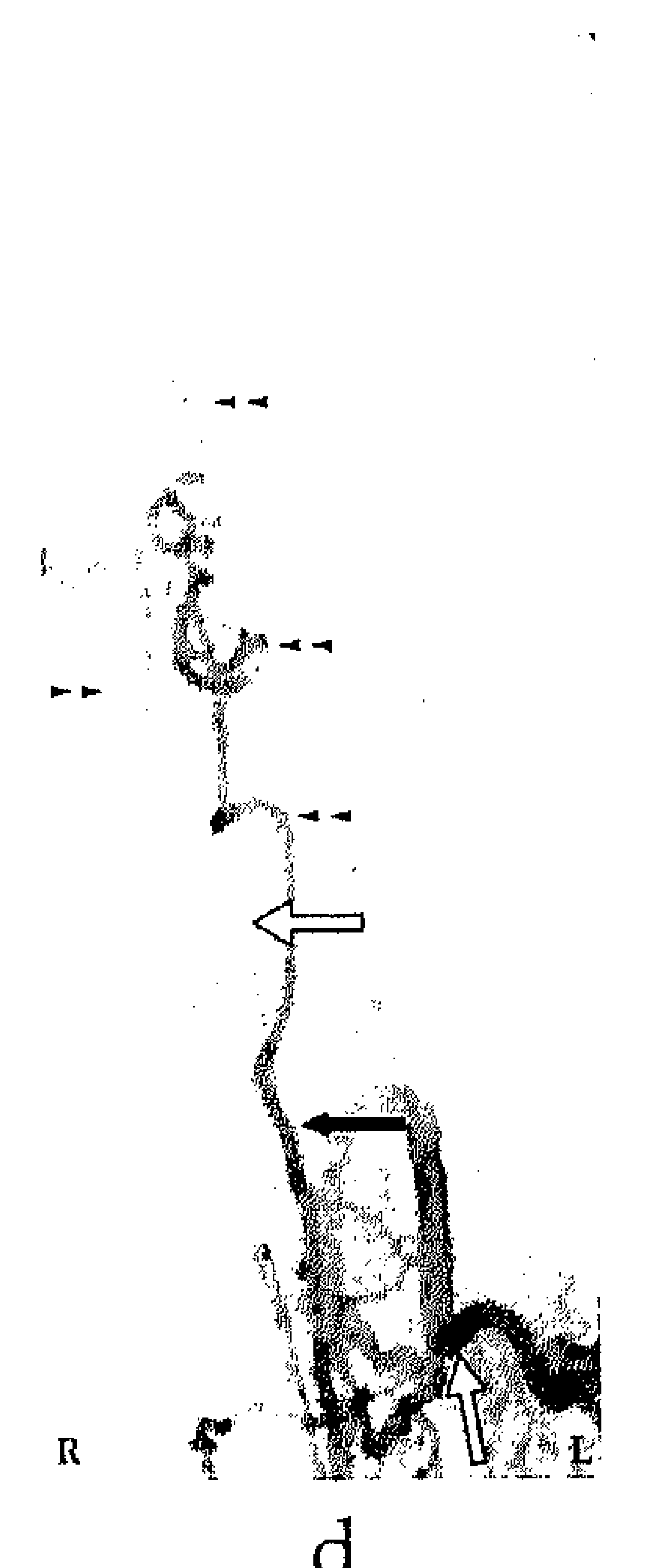

d

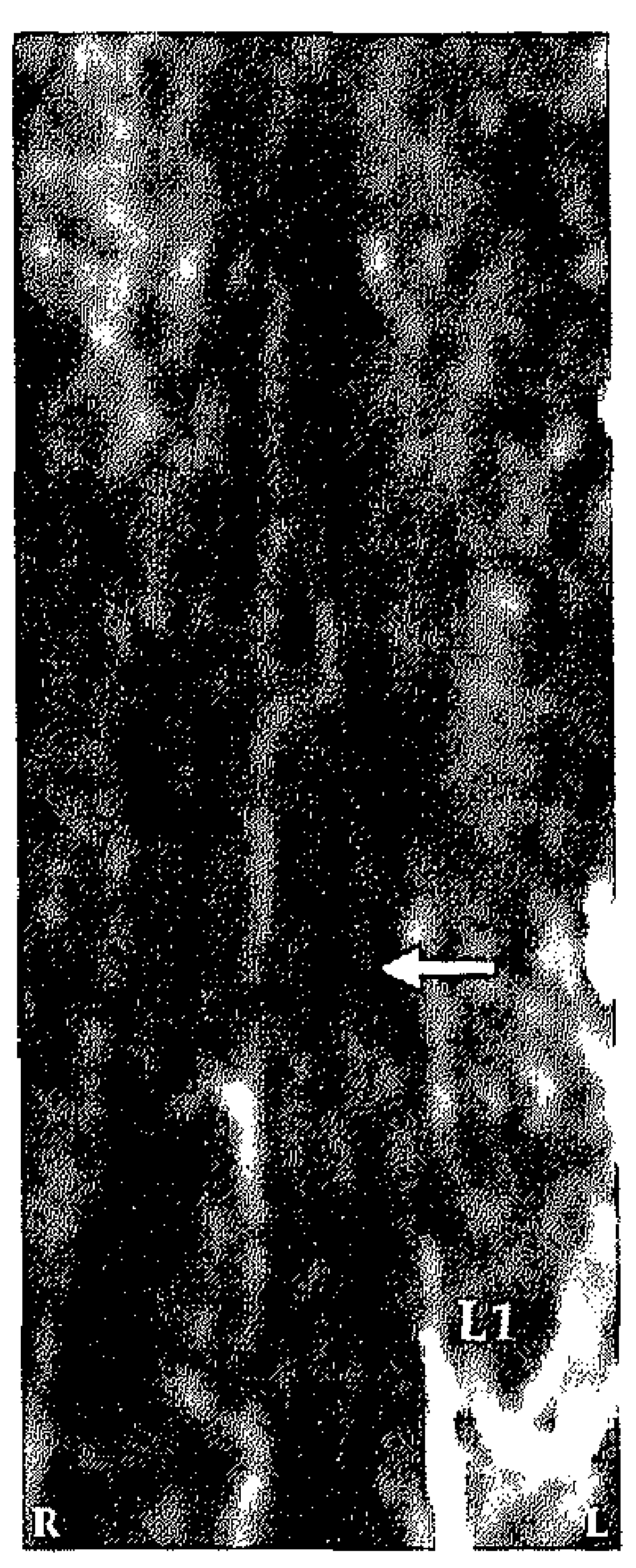

e

b

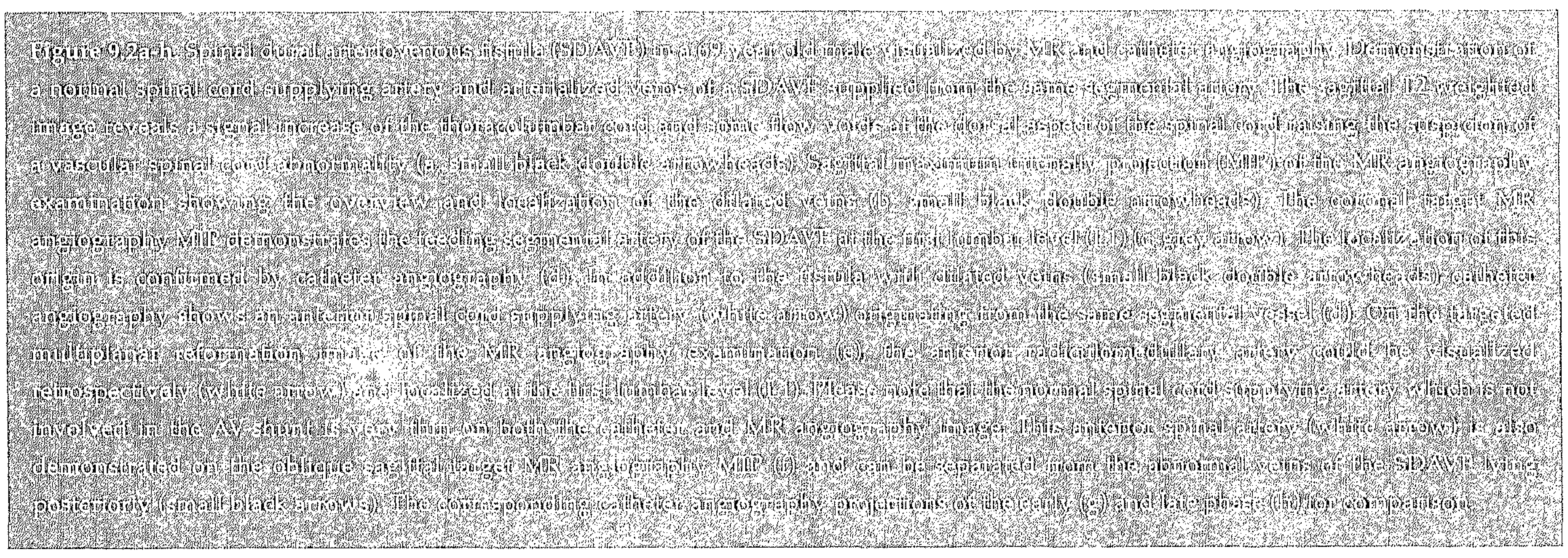




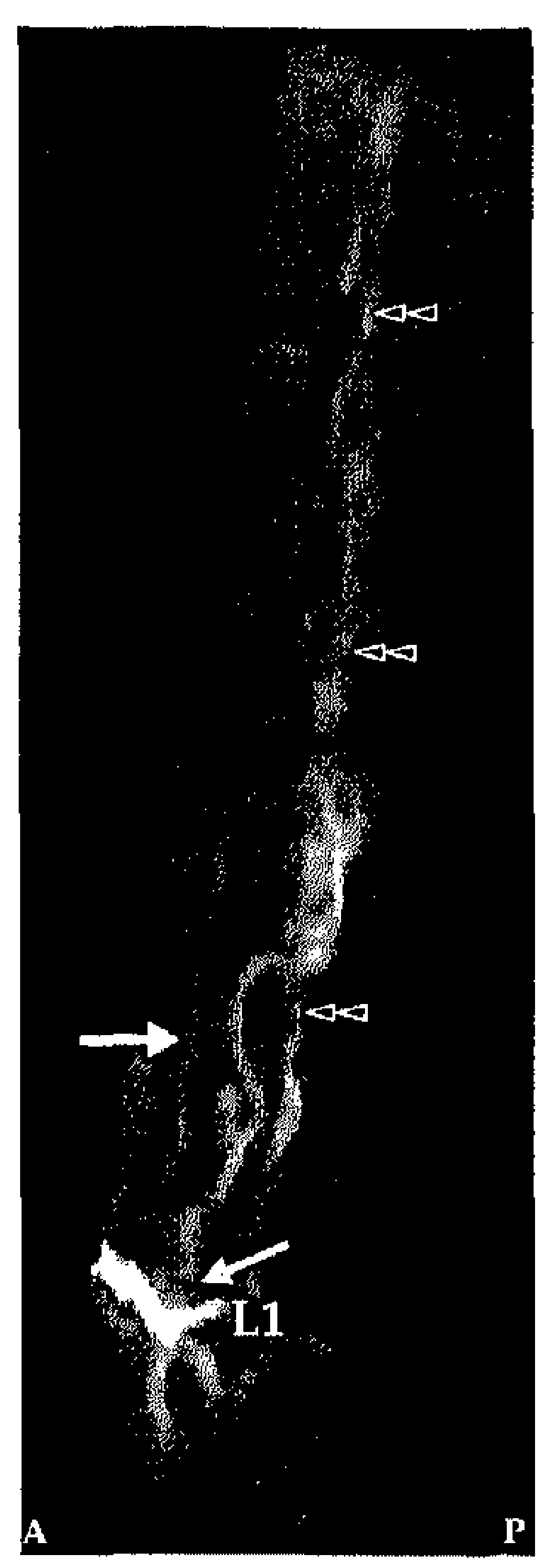

f

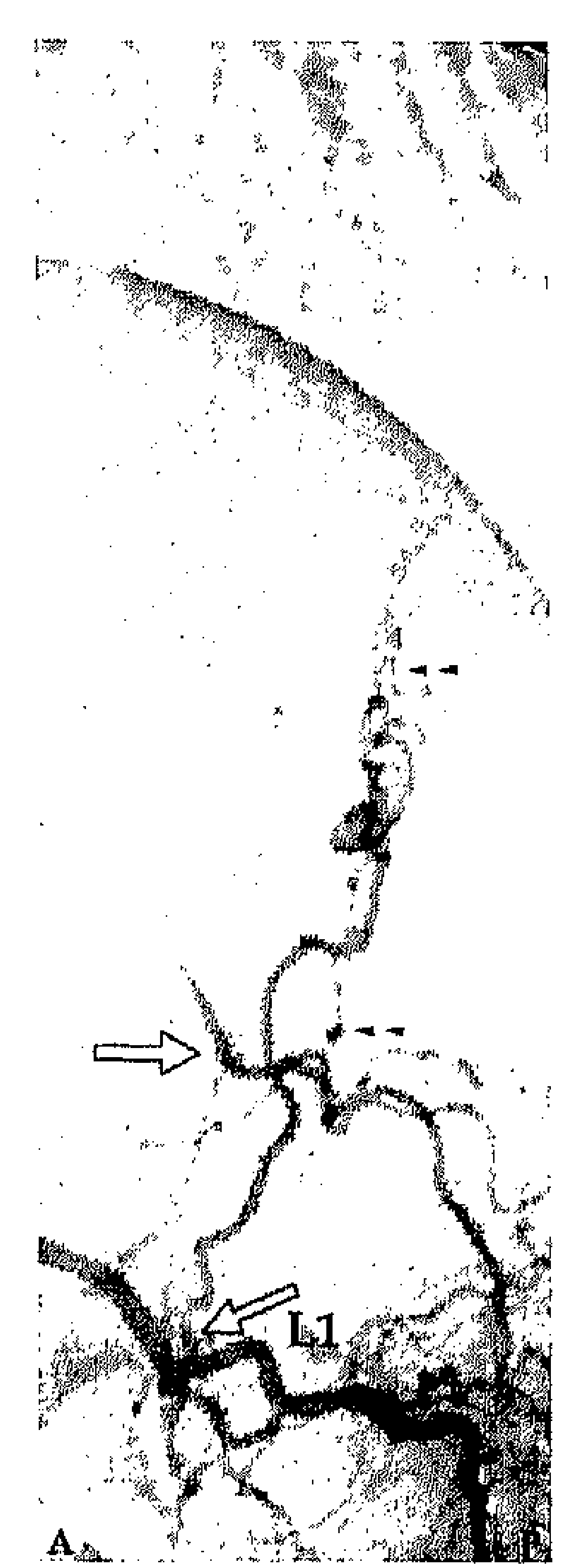

g

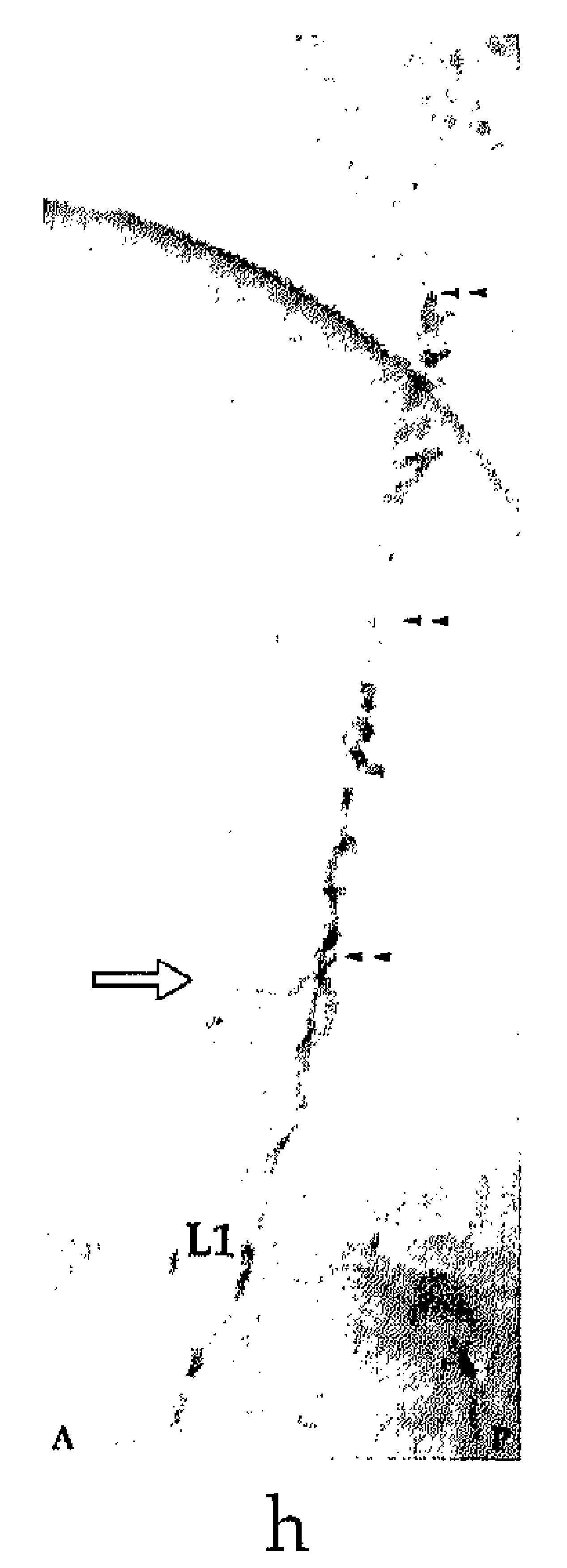

additional injections into both vertebral arteries, the costocervical arteries, the thyrocervical trunks, and the iliolumbar arteries was added. Imaging was in the anterior-posterior direction with 2 frames per second. X-ray parameters were $80 \mathrm{kV}, 400 \mathrm{~mA}$, matrix size $1024 \times 1024$, entrance format $11 \times$ $13 \mathrm{~cm}$ yielding a spatial resolution of 2.2 line pairs $/ \mathrm{mm}$. Oblique and lateral views were added to elucidate the morphology of the fistula zone in SDAVF and the composition of the nidus in SAVM. Film sequences of at least 5-20 seconds were taken, depending on the underlying vascular pathology were recorded, to evaluate the venous drainage.

In all cases of SAVM the catheter angiography examination included identification of all arteries supplying the thoracolumbar cord.

iodinated non-ionic contrast medium lumbar and intercostals arteries. If necessary, 


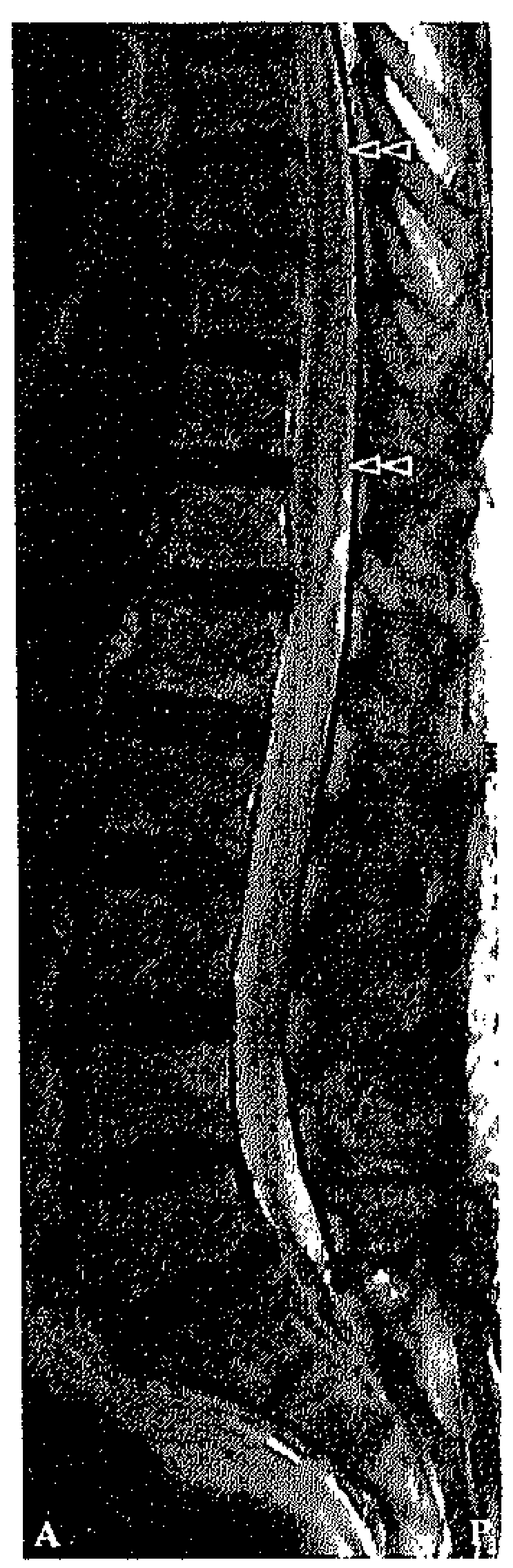

a

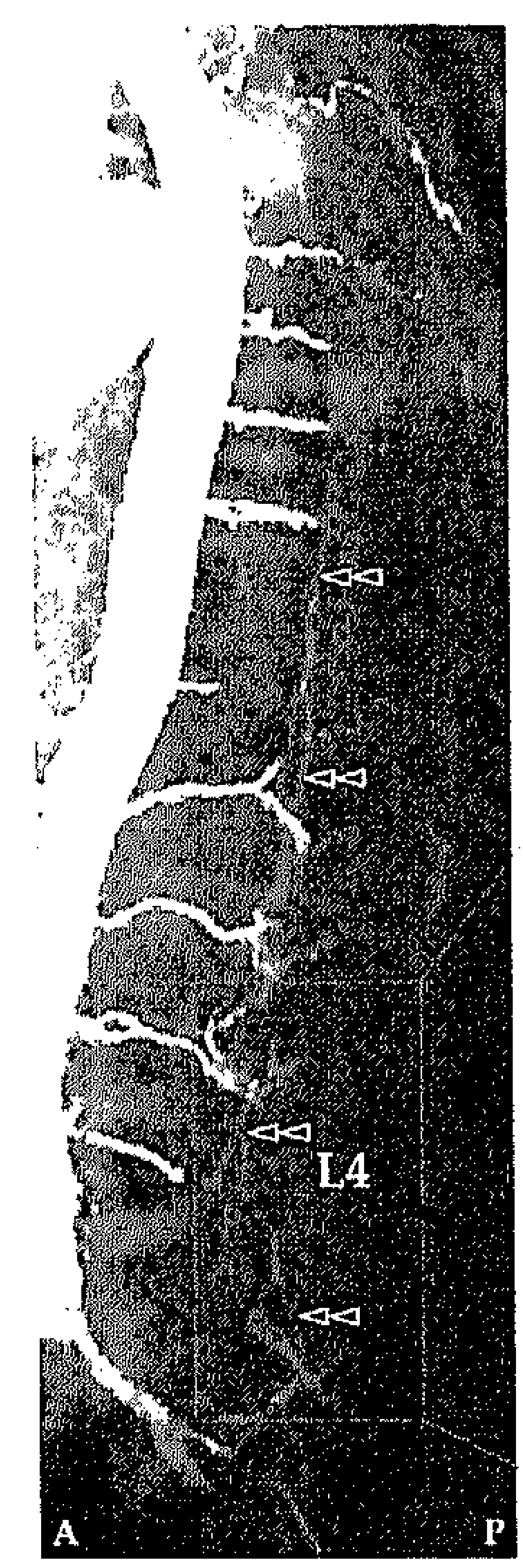

b

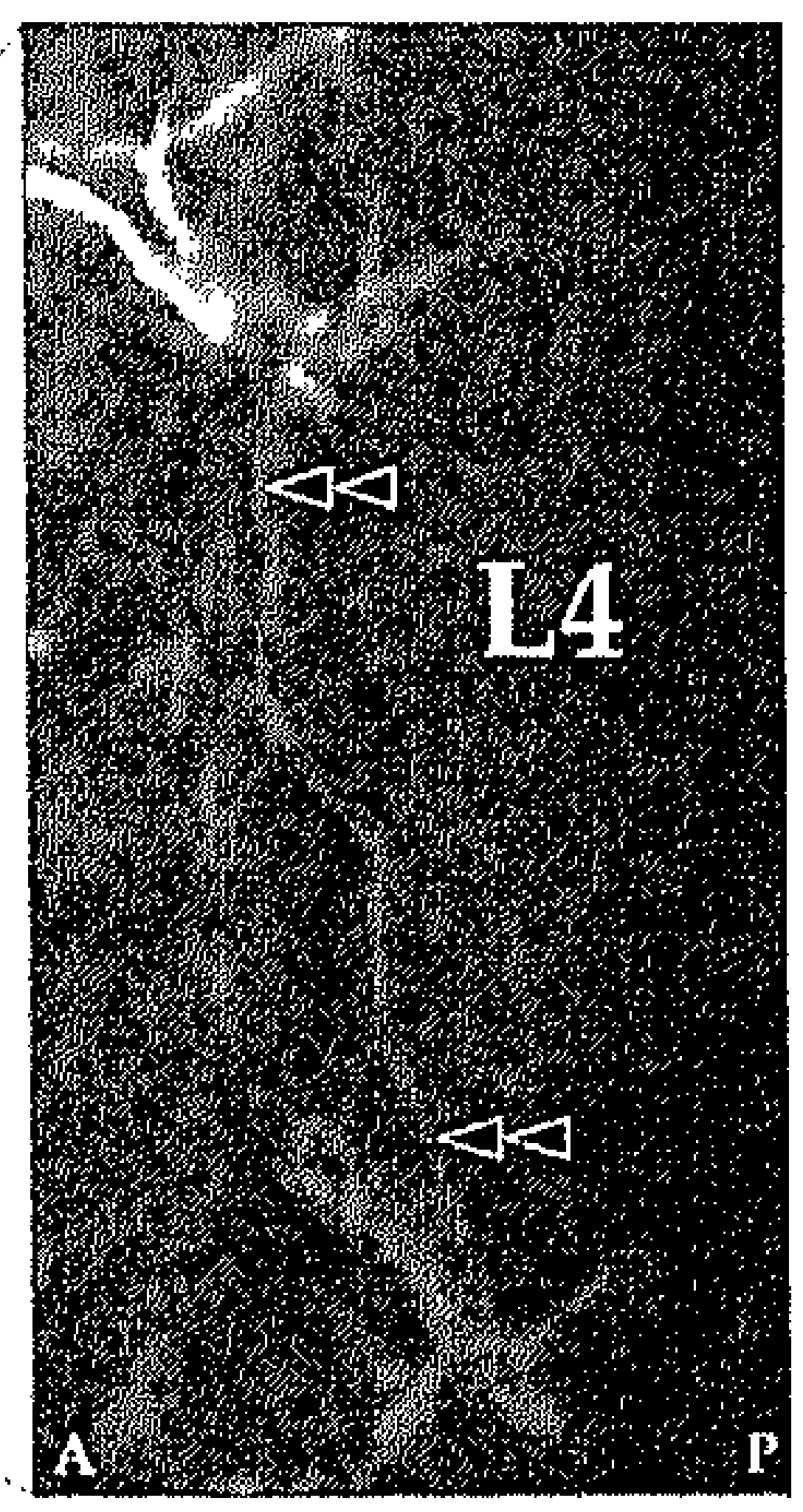

C

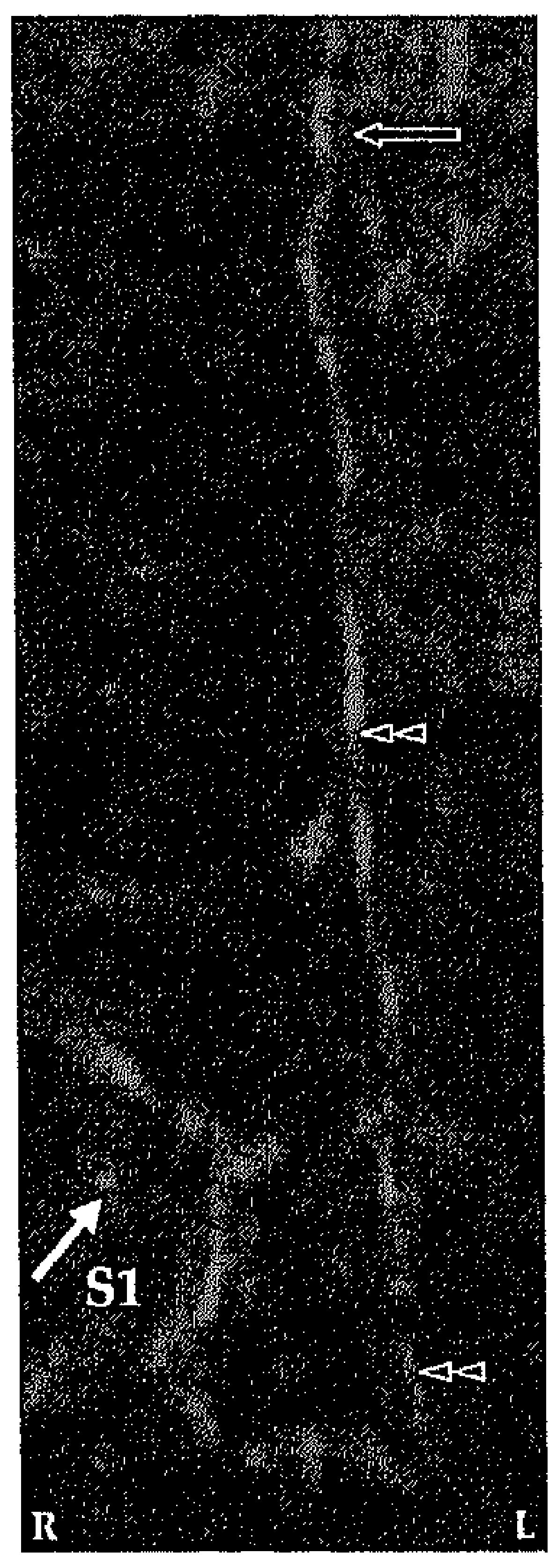

d

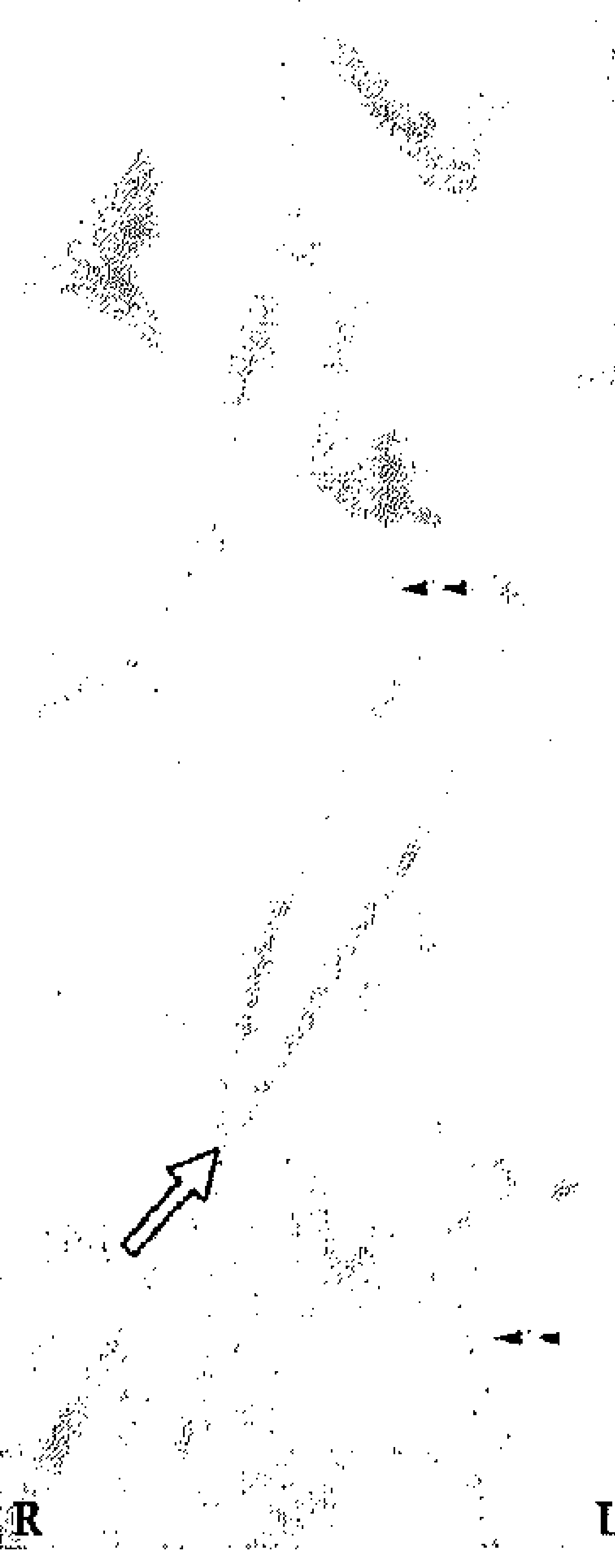

e

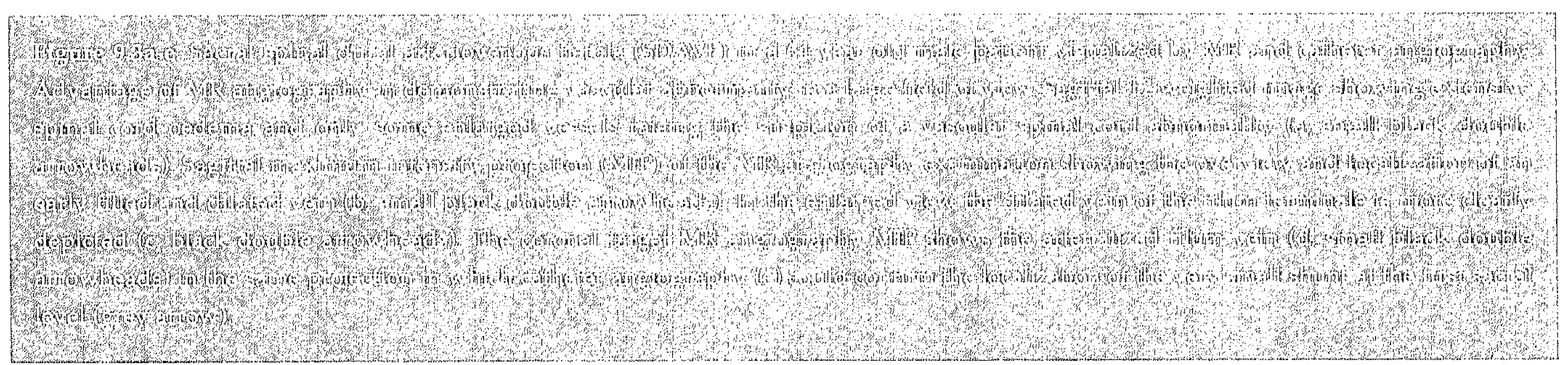

If the preceding $M R$ angiography examination suggested the level of a SDAVF, spinal catheter angiography included at least injection of the segmental arteries, on both sides, one level above and below the suggested level. Selective additional injections of the iliolumbar arteries were necessary in patients with suspected, spinal sacral AV shunt. In some patients with extensive thoracolumbar venous congestion complete spinal angiography was necessary with imaging of the cervical and upper thoracic spine via bilateral vertebral, subclavian, costocervical, thyrocervical, and carotid arteries. More than one diagnostic catheter angiography examination after MR angio- 
Table 9.2. Spinal Arteriovenous Malformation (SAVM) - Findings at MR and catheter angiography.

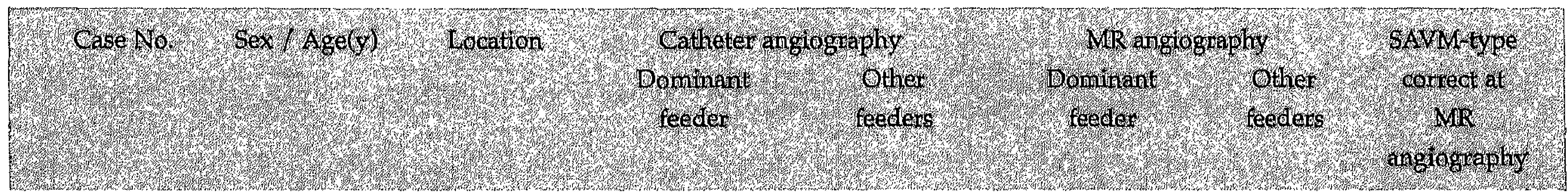

Glomerular type

$$
(n=3)
$$

\begin{tabular}{|c|c|c|c|c|c|c|}
\hline 21 & $\mathrm{~m} / 39$ & Thoracolumbar & $\mathrm{T} 121$ & T11 1, L11 & T12 1 & \\
\hline 22 & $\mathrm{~m} / 48$ & Thoracolumbar & $\mathrm{L} 1 \mathrm{r}$ & $\mathrm{T} 11 \mathrm{l}, \mathrm{T} 12 \mathrm{r}$ & $\mathrm{L} 1 \mathrm{r}$ & $\mathrm{T} 11 \mathrm{l}, \mathrm{T} 12 \mathrm{r}$ \\
\hline 23 & $\mathrm{~m} / 36$ & Thoracolumbar & T9 1 & L2 1 & T91 & L2 1 \\
\hline
\end{tabular}

Fistulous

type $(\mathrm{n}=3)$

Thoracolumbar
Thoracic
Thoracolumbar

L1 1

T5 r, T6r

$\mathrm{m} / 29$

Thoracolumbar

T11 $\mathrm{r}$

Filum terminale

$\operatorname{AVM}(n=5)$

$\begin{array}{ll}27 & \mathrm{~m} / 56 \\ 28 & \mathrm{~m} / 44 \\ 29 & \mathrm{~m} / 77 \\ 30 & \mathrm{~m} / 78 \\ 31 & \mathrm{~m} / 61\end{array}$

$54 / 5$

L 3

L 4

52

L 5 / S 1

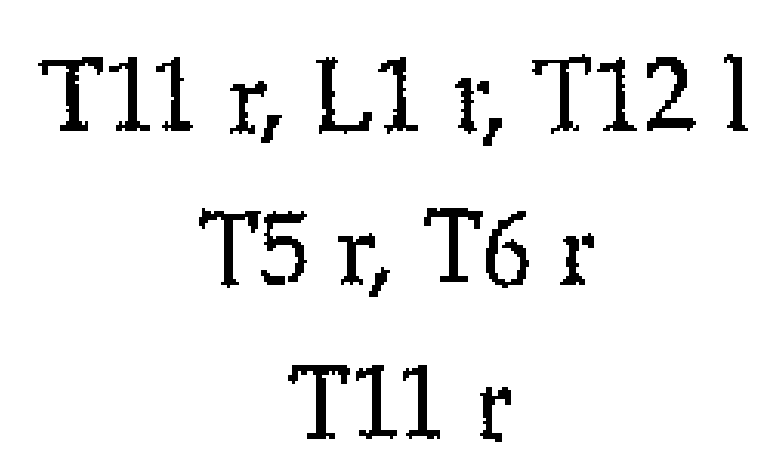

Iliaca interna I

L1 $\mathrm{r}$

T12 1

L1 1

L3 1

L4 $\mathrm{r}$
No

Yes

Yes

Yes
No
Yes

Yes

Yes

No

No

No

$\mathrm{m}=$ male; $\mathrm{f}=$ female; $\mathrm{T}=$ thoracic vertebra; $\mathrm{L}=$ lumbar vertebra; $\mathrm{S}=$ sacral vertebra; $\mathrm{r}=$ right; $\mathrm{l}=$ left

graphy was only required in those 3 patients who had negative MR angiography results.

\section{Image analysis}

The MR angiography images were postprocessed using maximum intensity projections (MIP) and curved multiplanar reformation (MPR). One investigator determined the level and side of the arterial feeder of the AV abnormality ${ }^{12}$ and subsequently provided digital images in which the meningoradicular artery supplying the SDAVF or the arterial feeder(s) of the SAVM were displayed optimally for the comparison study with catheter angiography. The MIP images comprised the early and late phases. Catheter angiography images were documented on plain films.

In all patients MR angiography images were analyzed prior to the catheter angiography examination. The report specified whether the MR angiography was positive or negative for a spinal AV abnormality. Moreover, the Adamkiewicz artery was localized ${ }^{13}$ and the spinal AV abnormality was characterized to differentiate between SDAVF, dural or epidural, and SAVM. Intradural SAVMs were further subcategorized in one of four groups 


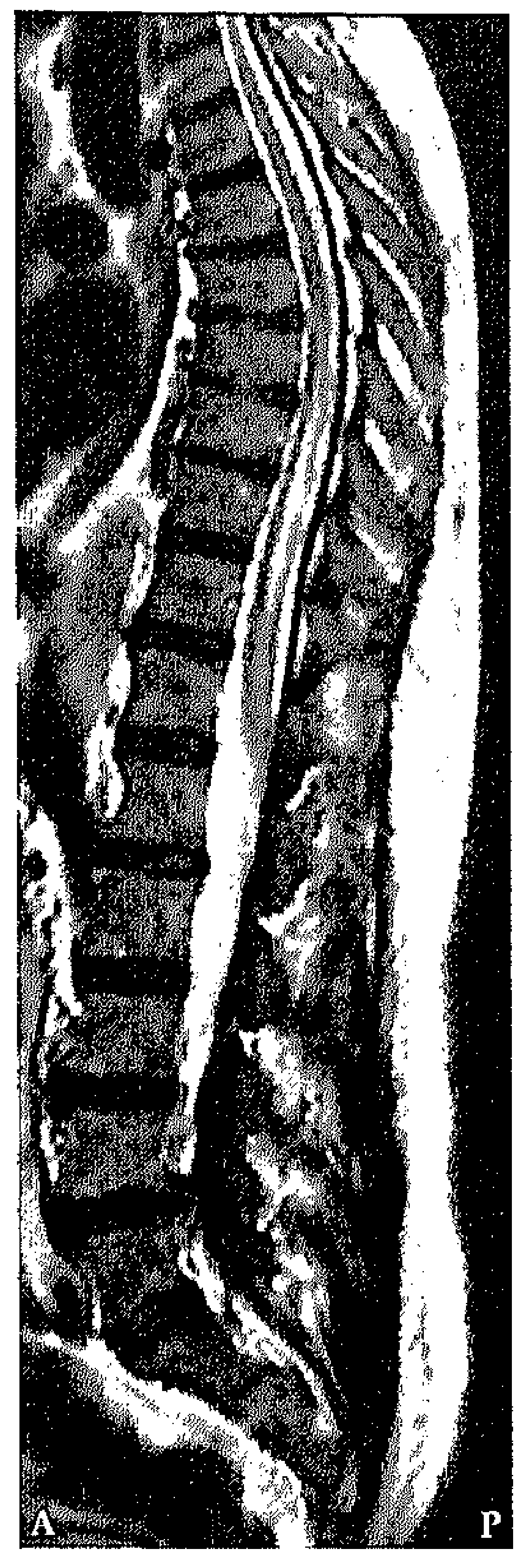

a

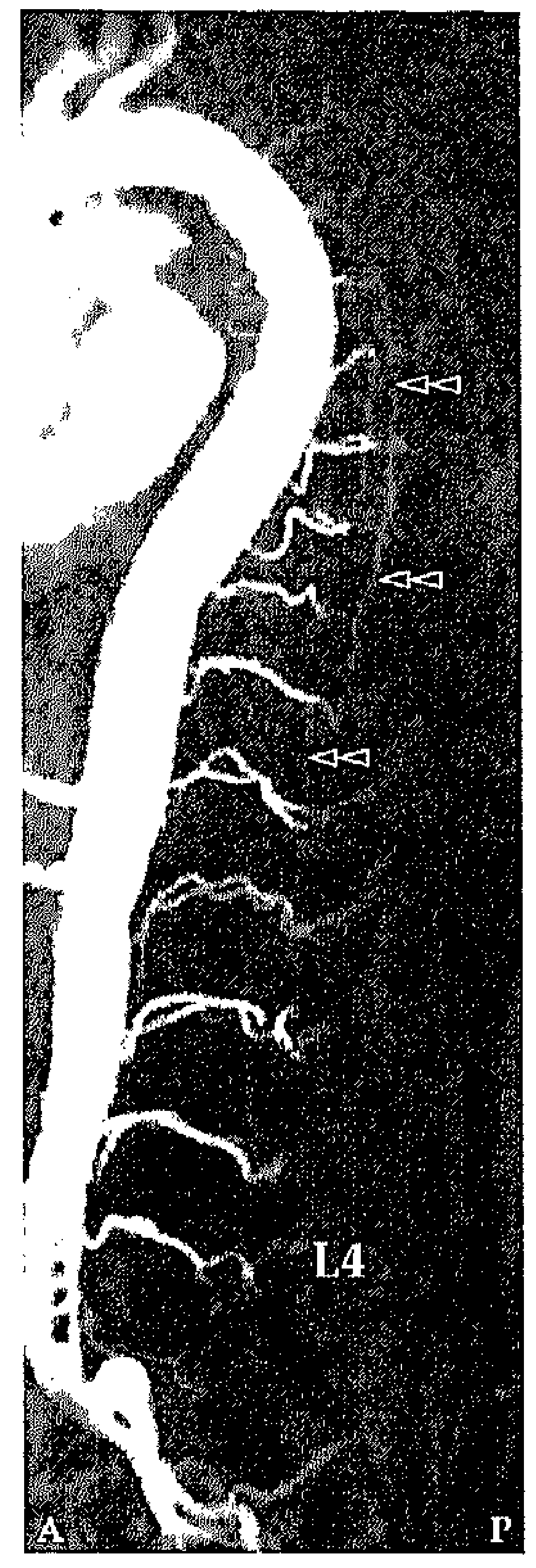

b
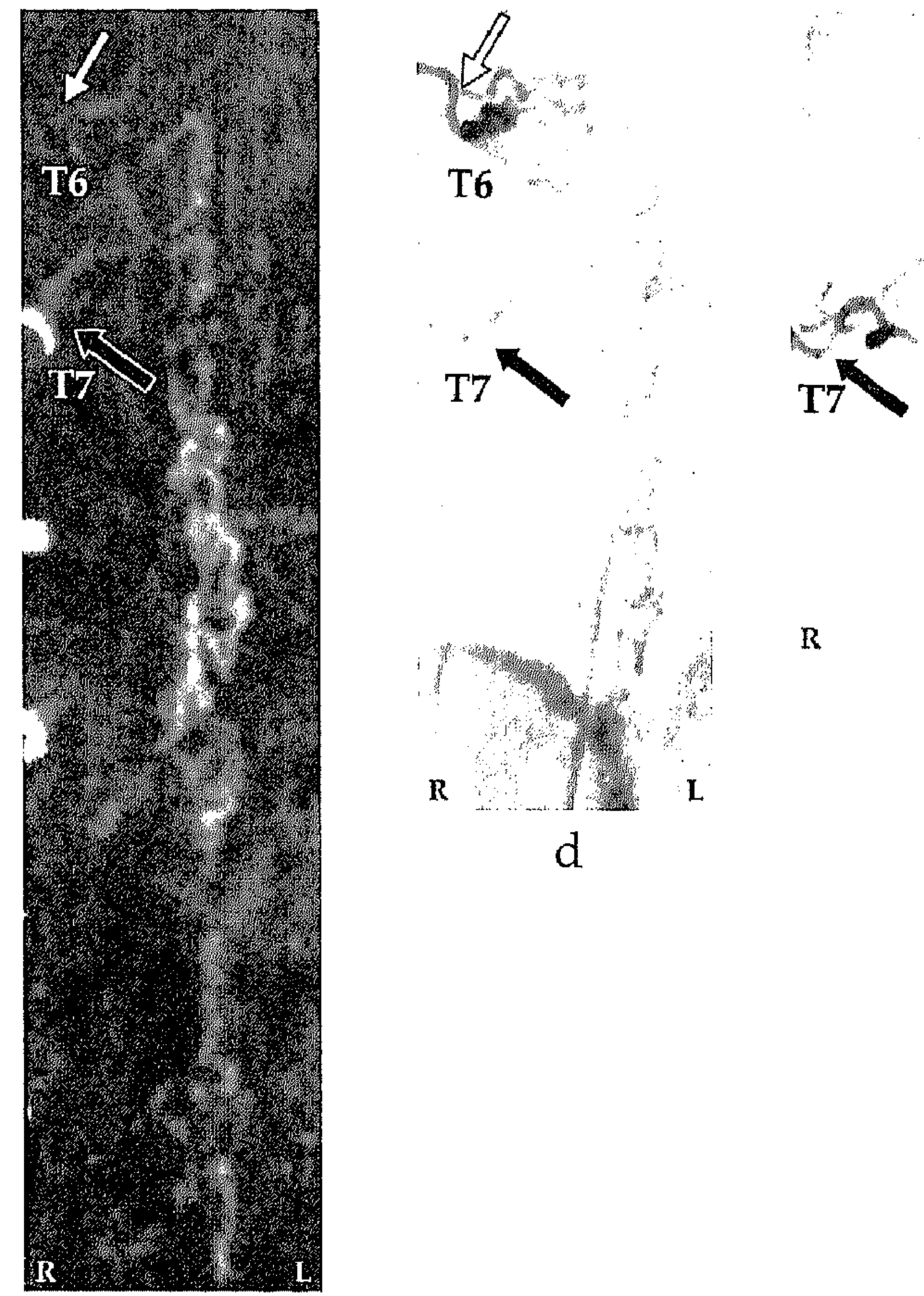

R

e

C

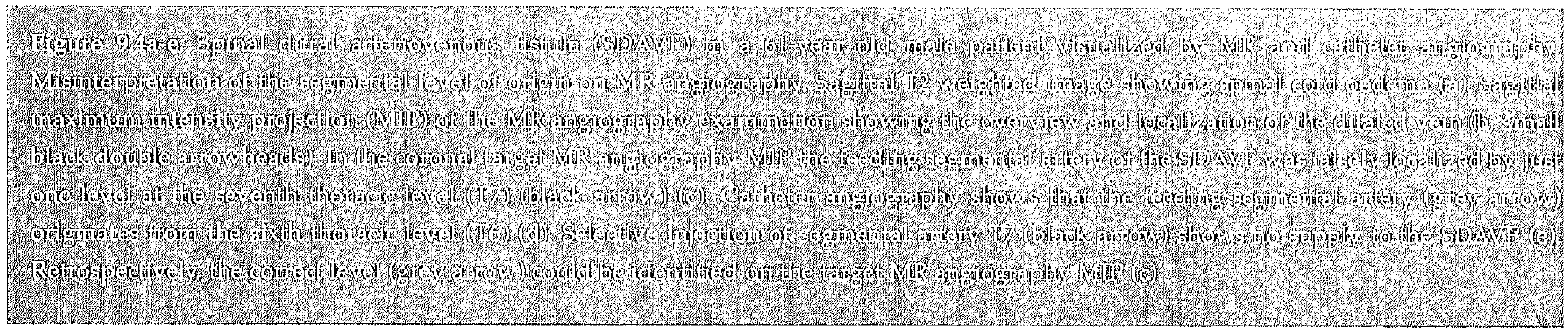


depending on the morphology, location, and hemodynamics of the AV shunt: (i) fistuloustype SAVM, (ii) glomerular-type SAVM, or (iii) SAVMs of the filum terminale. SAVMs on the filum terminale are characterized by an abnormally dilated anterior spinal artery and an extremely distant AV shunt located on the filum terminale. If $M R$ angiography was positive in suspected SDAVF the side and segmental level of the fistula and in cases of suspected SAVM the side and segmental level of the arterial feeders were searched for. When possible dominant and non-dominant feeders were discriminated in SAVM.

Definite localization and characterization of the vascular pathology was based on catheter angiography findings. Two neuroradiologists experienced with spinal vascular abnormalities evaluated the catheter angiograms for the presence and location of the fistula including level and side of the SDAVF. For SAVM, the feeding arteries were evaluated with regard to their level and side, their role in supplying the SAVM (dominant vs. nondominant) and whether the SAVM was glomerular or purely fistulous. Since pure glomerular or fistulous SAVMs are rare, the dominating feature defined the type of SAVM.

\section{RESULTS}

\section{Detection of $A V$ abnormalities}

AV shunts were present in 31 of 34 patients, of which 30 were spinal and 1 was tentorial with spinal drainage. MR angiography detected enlarged perimedullary blood vessels in all 31 cases with spinal abnormalities diagnosed by catheter angiography. No spinal vascular abnormalities were found in 3 patients, 2 of whom had a spinal subarachnoid hemorrhage and 1 had extensive thoracic cord oedema suspected to be due to venous congestion. In these 3 patients complete catheter angiography required up to four sessions because of the limitations on the maximum dosage of the contrast medium. No false positive $M R$ angiography results were observed in these 3 patients.

In 1 patient a tentorial DAVF was finally encountered. In this case the location of the fistula could not be identified by MR angiography. The extent of the venous drainage visualized by MR angiography suggested that the fistula was located cranially to the field of view of MR angiography. The subsequent catheter angiography examination demonstrated a tentorial DAVF with extensive spinal perimedullary venous drainage.

One patient was classified with an epidural AVF. The level of the arterial lumbar feeder in this patient could be defined after MR angiography pointed to the correct level resulting in a successful catheter angiography examination.

\section{Localization of SDAVF}

In the 19 patients with SDAVF with retrograde perimedullary venous spinal drainage MR angiography correctly predicted the level of the fistula within one vertebral level in all cases (Table 9.1 \& Figures 9.1-9.4). Even deeply located sacral fistulas were correctly identified by MR angiography prior to catheter angiography (Figure 9.3). In five cases a mismatch of one vertebral level (not side) was noted (Figure 9.4). Possible reasons for these mismatches were the poorly visualized proximal part of the draining vein (patients 6, 14, and 16) or variants of the segmental arterial supply and the bony structures (patients 15 and 17). 


\section{Arterial feeders in SAVM}

Eleven patients were identified with a SAVM, which were localized in the thoracolumbar region in 6 cases (Figure 9.5) and on the filum terminale in 5 cases (Figure 9.6). Fistulous-type SAVMs were found in 3 patients. Five patients had an AVM of the filum terminale. Three patients had a glomerulartype SAVM with a distinct nidus of abnormal vessels between the arterial and venous side. This nidus could be clearly seen in the parenchymal MR image. MR and catheter angiography findings are listed in Table 9.2.

The main arterial feeder could be identified in 10 out of 11 cases of SAVM with MR angiography. There were 5 patients with additional feeders demonstrated by catheter angiography. MR angiography could only depict additional feeders in 2 patients (case $22 \& 23$ ).

In the thoracolumbar region differentiation between glomerular and fistulous SAVM was successful with MR angiography in 4 out of 6 cases. For the filum terminale SAVM the dilated main arterial feeder was found in 5 of the 5 cases. However, classification of this rare type of abnormality by MR angiography was only possible in 2 cases. In the remaining 3 cases catheter angiography was necessary to correctly classify this abnormality.

\section{DISCUSSION}

\section{Current findings}

This study has shown to which extent MR angiography may serve to detect, localize, and characterize various types of spinal AV shunts in patients with suspected AV malformations, with selective spinal angiography as the reference study. The most important finding of this study was the observation that no false

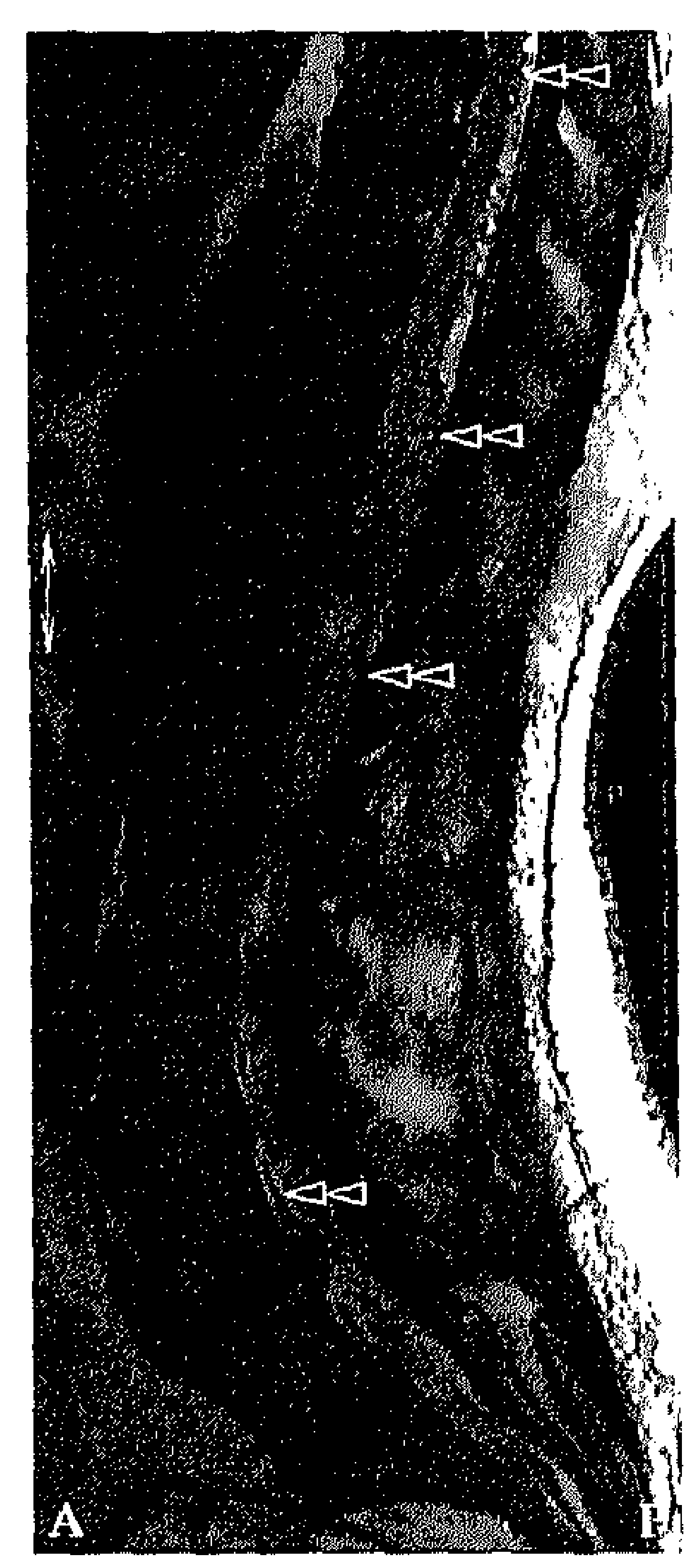

a

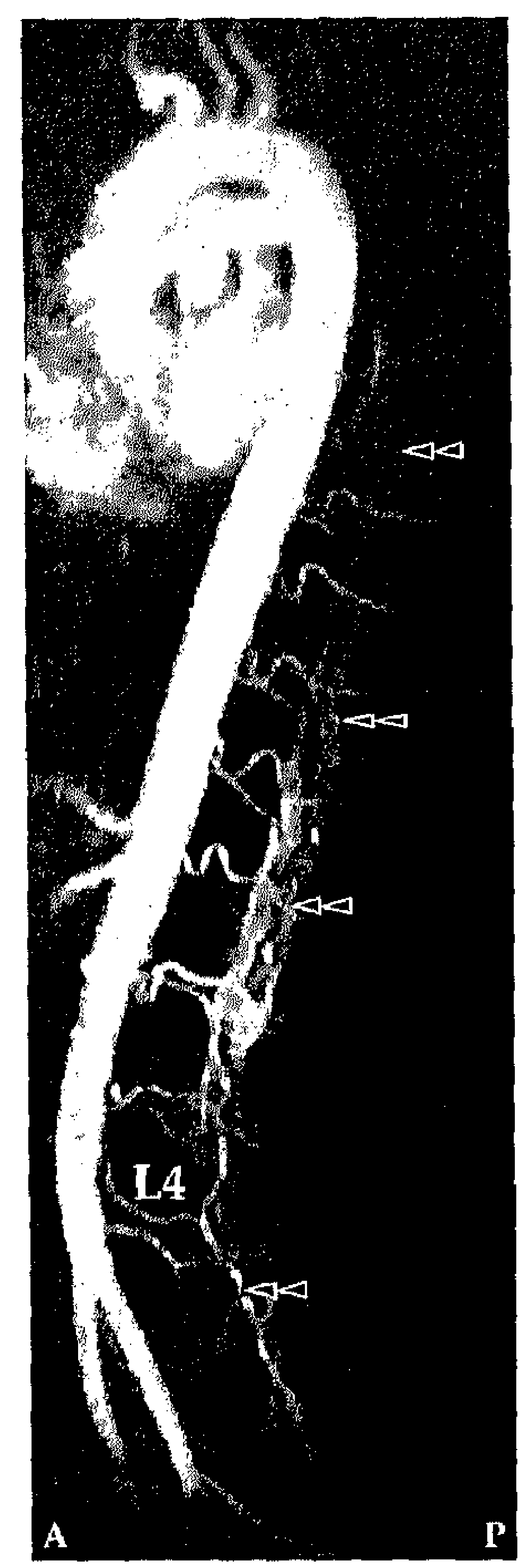

b positive and no false negative MR angiography results were obtained regarding $A V$ shunts of the spinal cord in a relatively large series of patients that harbour a rare disease. Consequently, as detection or exclusion of spinal AV abnormalities by MR angiography was found to be very accurate for both SDAVF and SAVM, it may in future be possible to avoid burdensome diagnostic catheter angiography procedures. Furthermore, MR angiography may substitute catheter angiography for follow-up diagnostic procedures. 

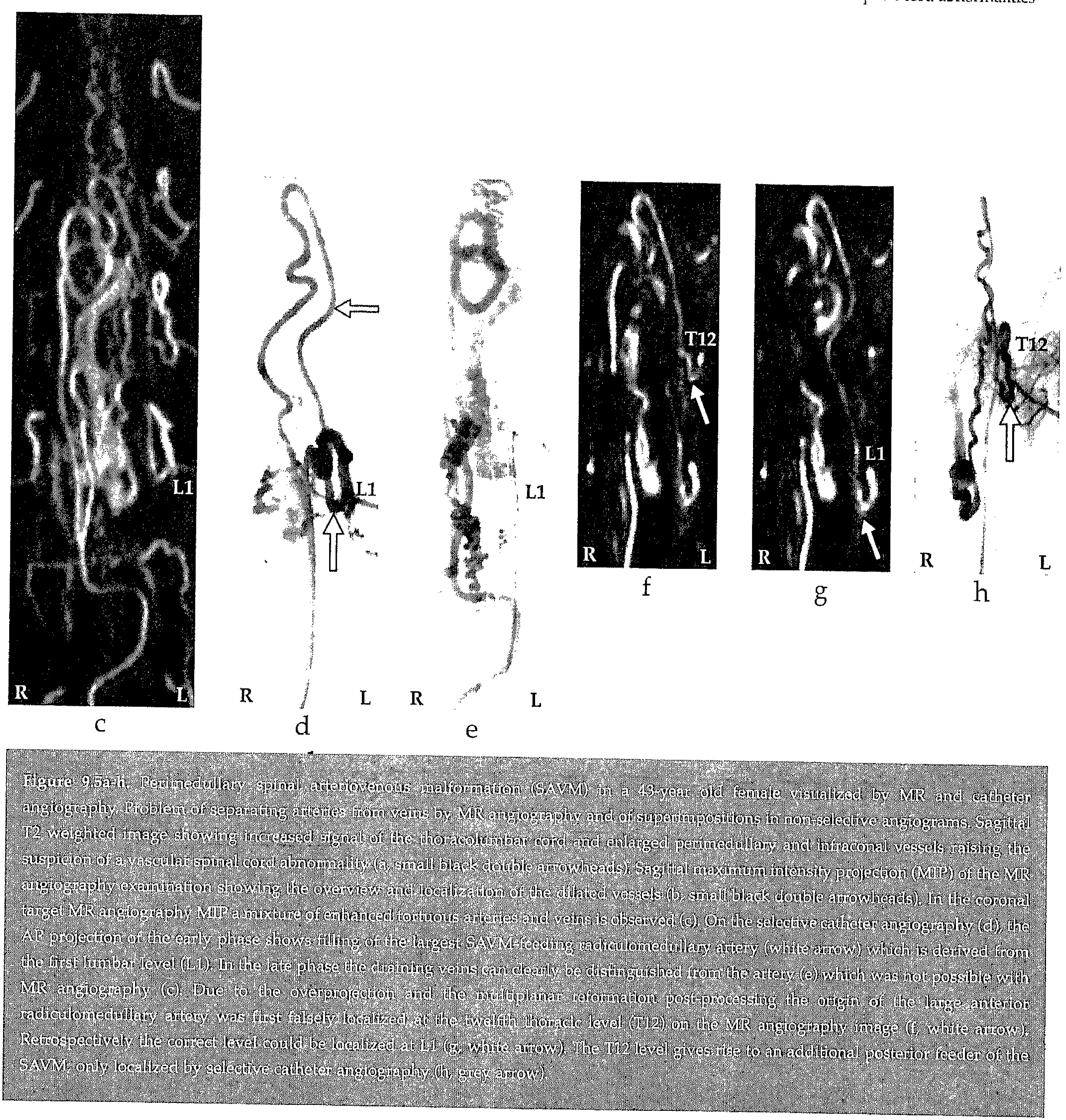


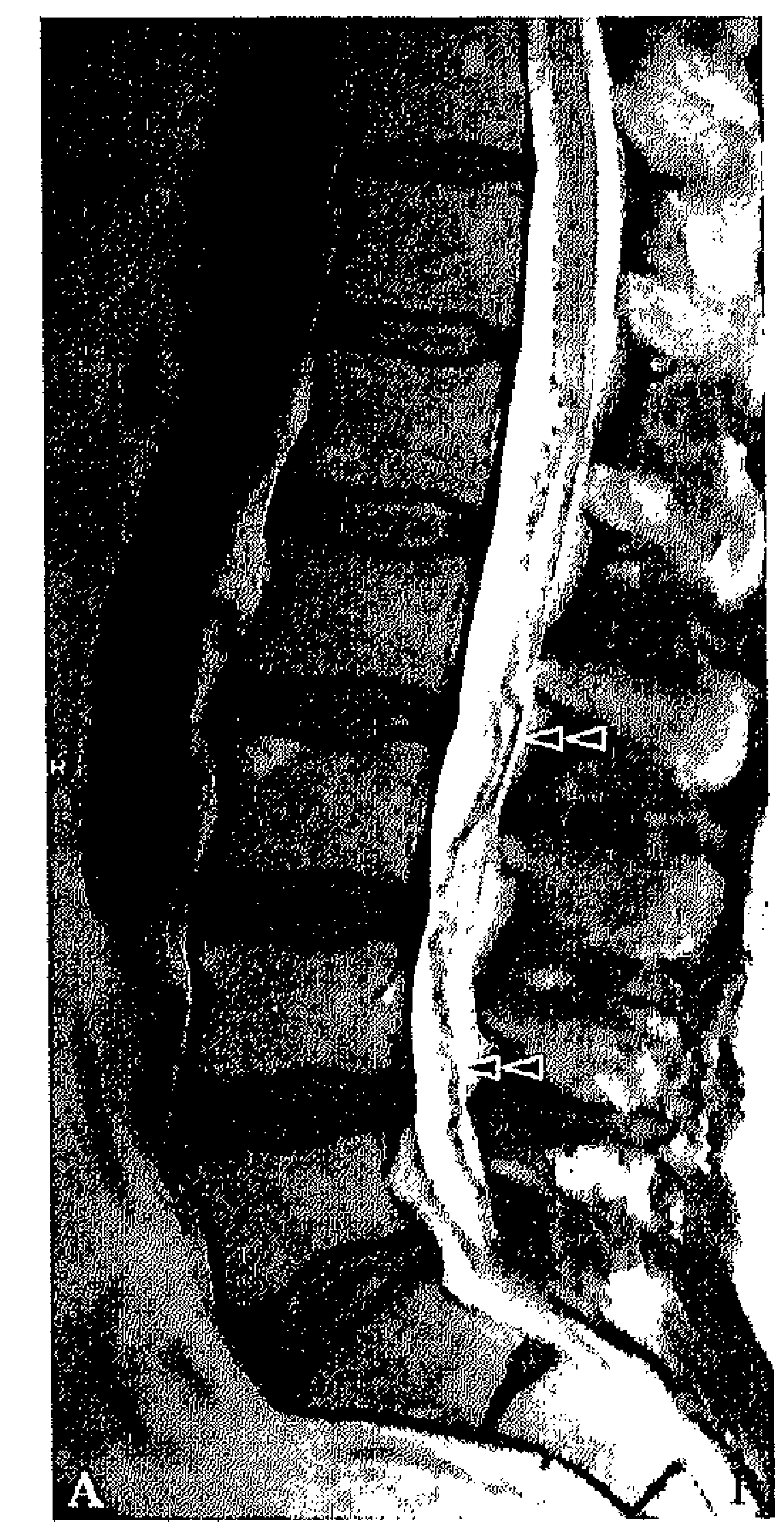

a.

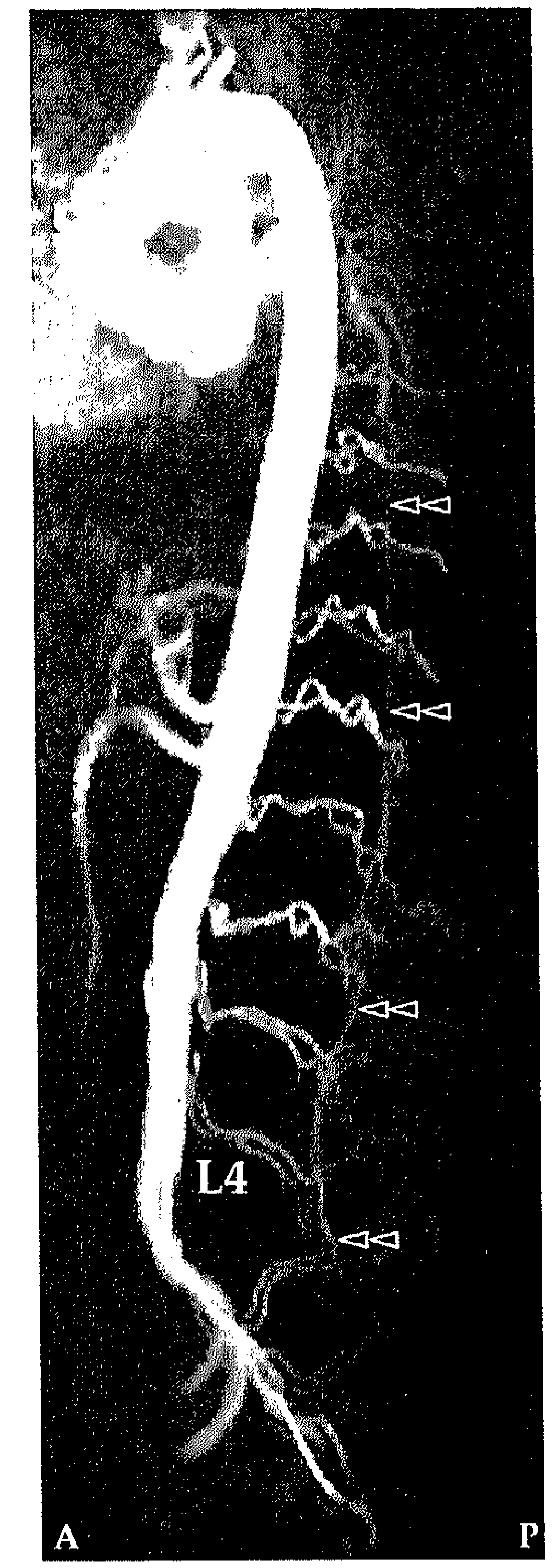

b

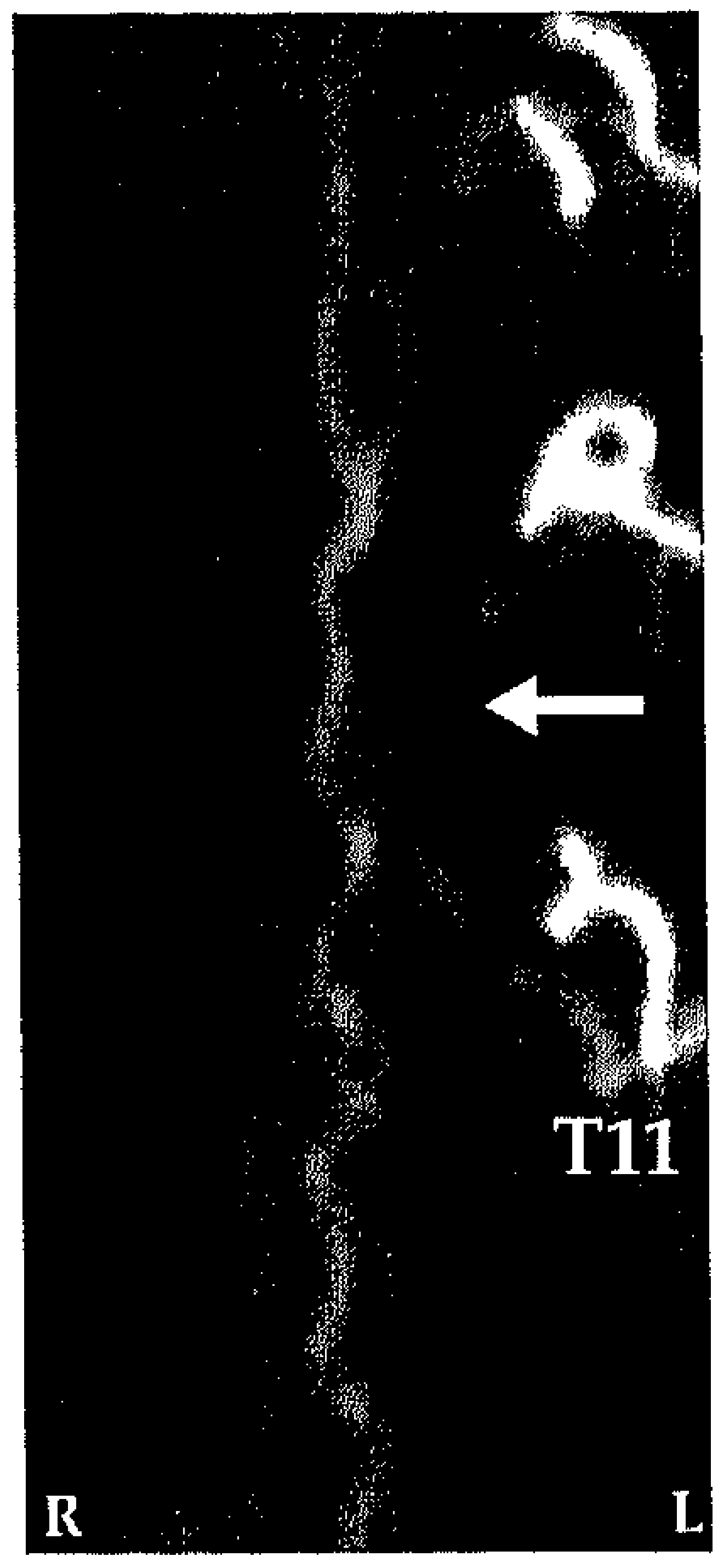

C

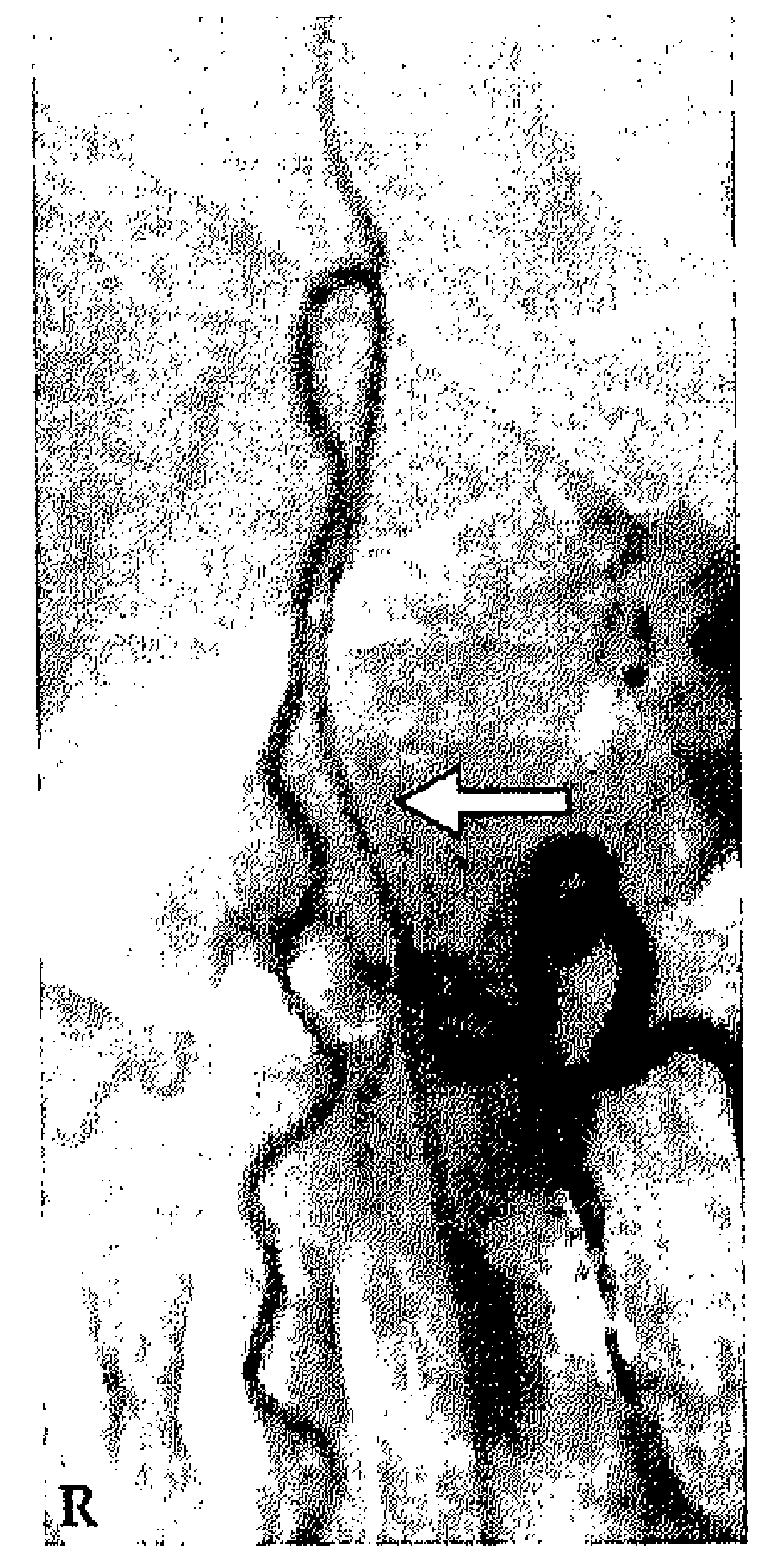

d

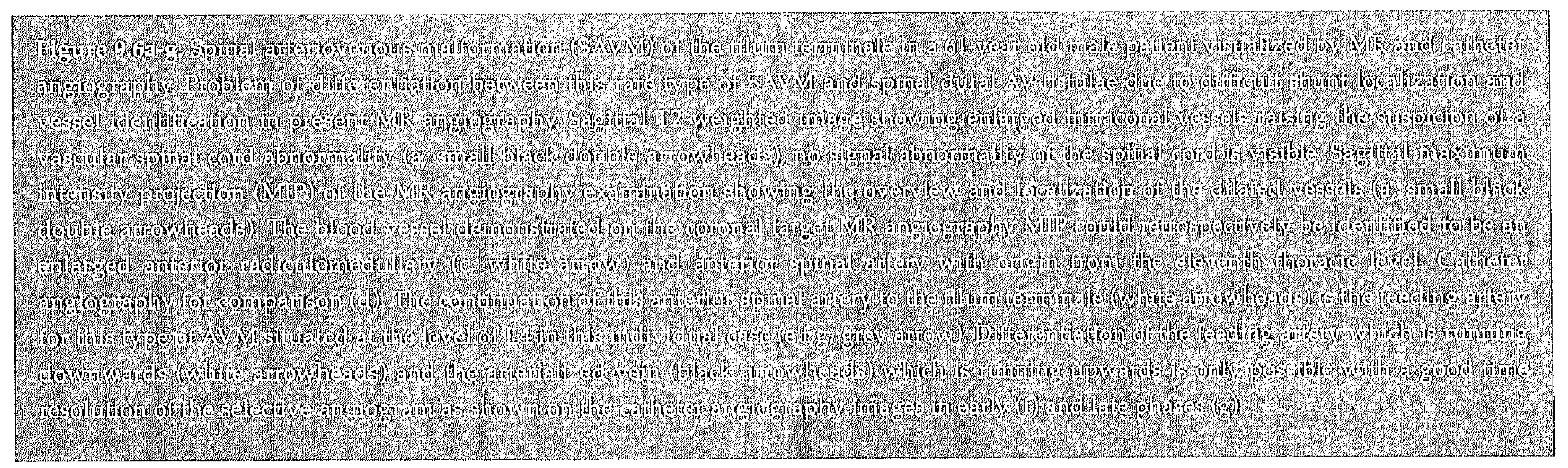




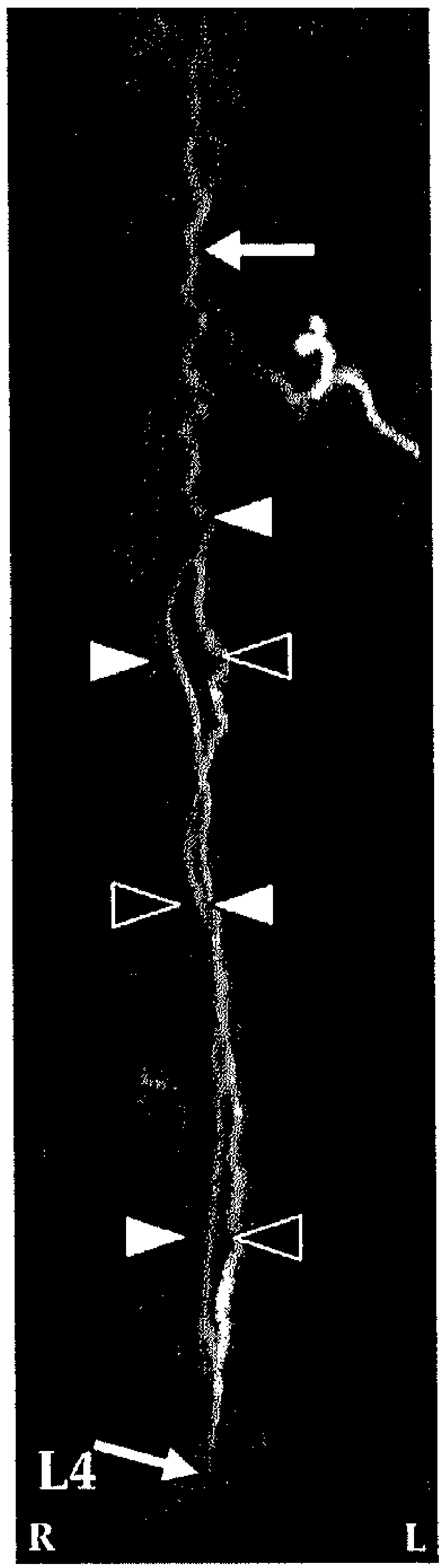

e

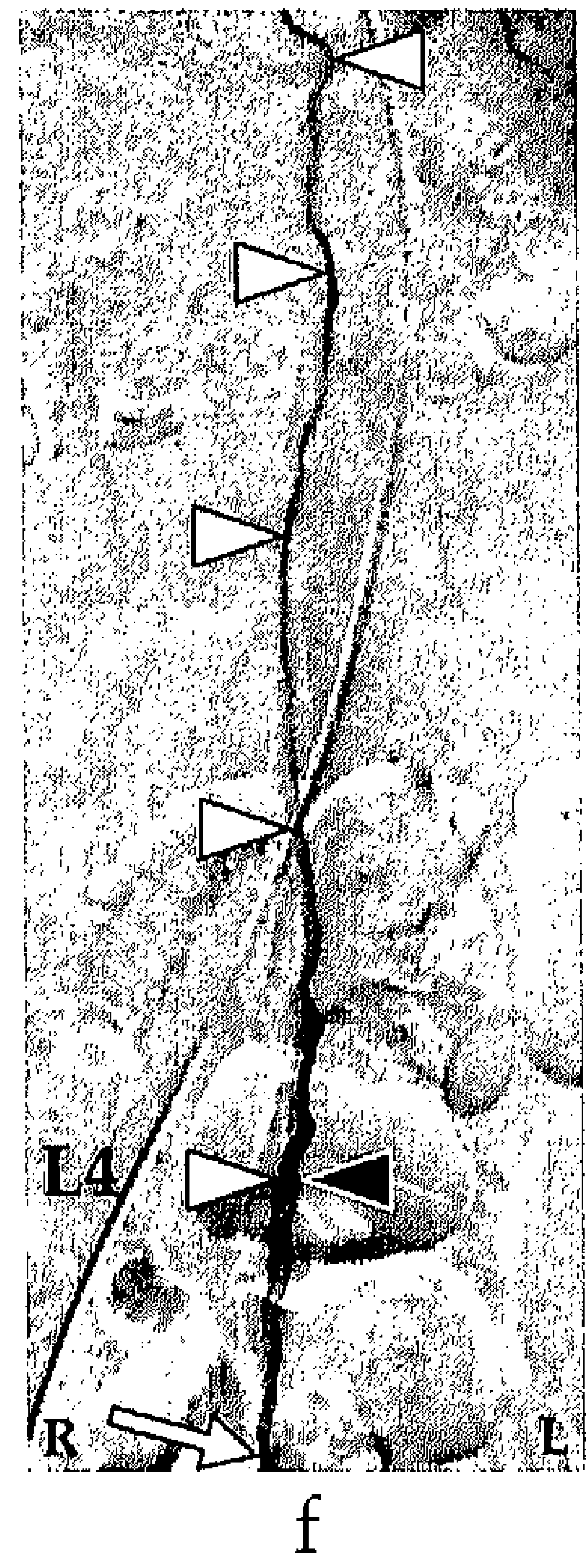

One may argue that one patient, who had a (cranial) tentorial dural AV fistula, was not correctly diagnosed by $M R$ angiography. However, MR angiography visualized the associated pathologically arterialized veins and guided the subsequent catheter angiography examination to the cranium. In this scan protocol we did not include the cervical spine for two reasons: (i) there is a technical restriction in the number of coil elements that

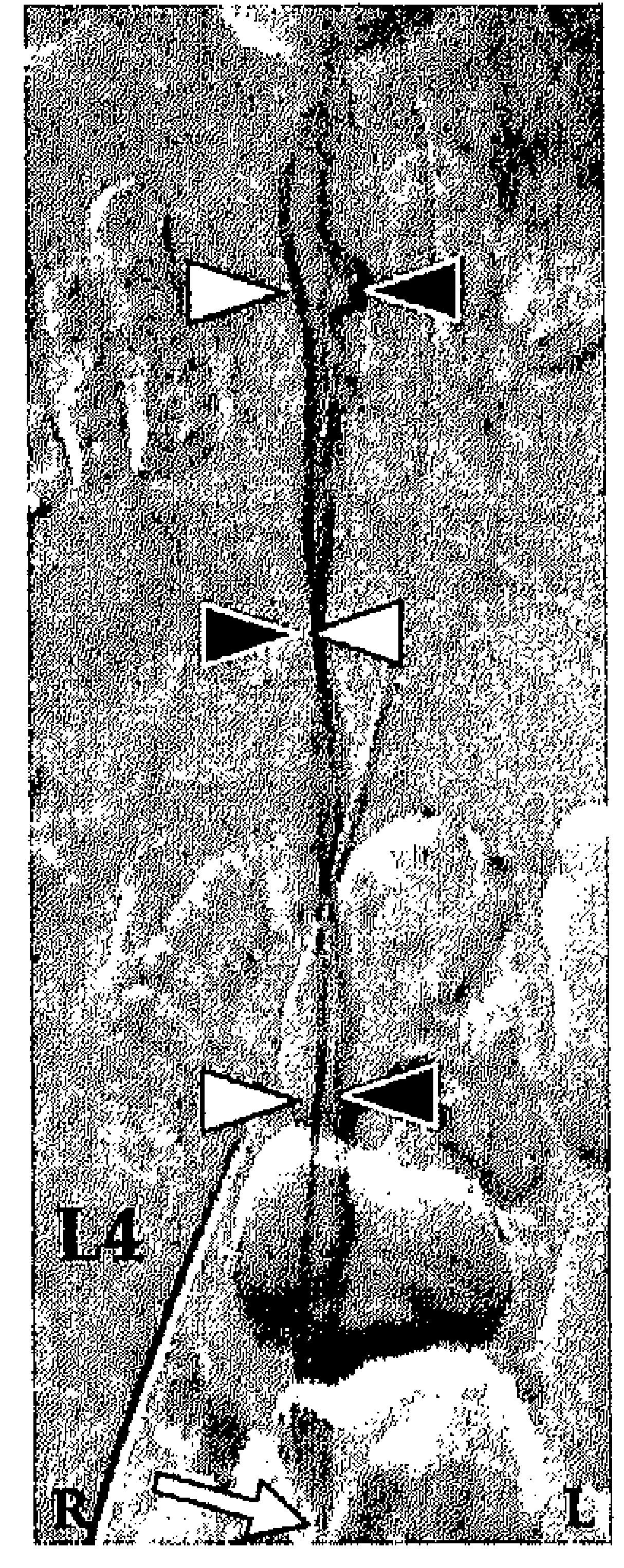

g

can be used at once and (ii) SDAVF of the cervical spine are exceedingly rare $^{3}$. Therefore we opted to use the elements that covered the entire thoracic, lumbar, and sacral region.

\section{Diagnostic spinal cord MR angiography}

The possibility of MR angiography to identify spinal cord vascular pathology was recognized previously in a number of studies. Mascalchi et $a^{8}$ found PCA suitable for the 
detection of SDAVFs and the arterial feeders of SAVMs in a high percentage (up to 12 out of 15 patients) of patients. Detection of draining veins, localization of the fistula and of normal arteries was however limited or in some cases not possible due to their low blood flow or small dimensions. Bowen et $\mathrm{al}^{9}$, on the other hand, using contrast-enhanced 3D steady-state MR angiography were able to visualize the dilated spinal cord veins associated with SDAVFs. Although these pathologic veins pointed towards the site of the AV shunt, the shunt itself was difficult to depict and normal intradural arteries were not observed. The main reason for this appears to be the fact that the contrast material was distributed over the relatively large veins and adjacent (background) tissue, which obscured the visualization of the small arteries and the shunting zone. Binkert et al $^{10}$ avoided contaminating venous and background enhancement by applying a fast (24 seconds) contrast-enhanced MR angiography technique and showed in a limited number of patients that the localization of the feeder of SDAVFs (2 out of 3 patients) and the classification of SAVMs ( 6 out of 6 patients) was feasible.

Recently, the studies of Farb et al ${ }^{11}(n=9)$ and Luetmer et $\mathrm{al}^{6}(\mathrm{n}=31)$ demonstrated, that SDAVFs can be accurately localized using an MR angiography technique, in which the arterial-venous contrast was emphasized with a bolus injection of contrast agent similar to our technique. Unfortunately, the craniocaudal field of view was limited $(28 \mathrm{~cm}$ and $36 \mathrm{~cm}$ respectively) thereby missing the level of the fistula in 5 cases in the study by Farb et al11 and in 6 cases in the study by Luetmer et $\mathrm{al}^{6}$. In neither study described above, it was possible to demonstrate normal intradural arteries, due to their small calibres of $1 \mathrm{~mm}$ or less, and owing to the course which is similar to perimedullary and radicular veins.

In the present study we have improved on these previous techniques by consistently visualizing the Adamkiewicz artery and its continuation in the anterior spinal artery by the temporal separation of early phases (arteries and arterialized veins) from relatively late phases of enhancement (arteries and veins together). Moreover, using our technique it was demonstrated that it is possible to visualize even small abnormal blood vessels of the lumbosacral spine in patients with SDAVF and SAVM of the filum terminale.

The MR angiography technique used in this study is similar to the one previously used for the preoperative detection of the Adamkiewicz artery in patients about to undergo thoracoabdominal aortic aneurysm surgery ${ }^{14}$. The relevance of detecting the Adamkiewicz artery in spinal AV shunts is illustrated by the case demonstrated in Figure 3 , in which the radiculomeningeal artery supplying the SDAVF also gave rise to the Adamkiewicz artery. For this case cautious treatment is required to avoid interruption of the blood supply to the Adamkiewicz artery, as there is increased risk for developing paraplegia.

The combination of a relatively large craniocaudal field of view $(50 \mathrm{~cm})$, a high gadolinium dose $(0.3 \mathrm{mmol} / \mathrm{kg})$ administered as a bolus, subtraction with noise-reduced precontrast images, and the synchronization of central k-space sampling to the contrast bolus arrival provides a more complete diagnostic examination in which nomal and abnormal arteries and veins can be distinguished, the AV shunt zone can be visualized, the predominant arterial feeders can be localized, and the venous drainage pattern can be displayed in a single MR angiography examination. 


\section{Limitations}

Despite these improvements there are limitations. Detection of multiple arterial feeders in cases with extensive fistulous SAVM remains limited to the largest feeders. Small additional feeders remain unnoticed with MR angiography. Although MR angiography was able to detect AV abnormalities of the sacral spine, differentiation between a sacral SDAVF or filum terminal SAVM was not possible in all cases. Likewise, subclassification of SAVMs in terms of glomerular and fistulous was not successful in all cases and still requires catheter angiography.

It should be stressed that the reliability of MR angiography for the purpose described in this study is strongly dependent on the quality of postprocessing (i.e., multiplanar reformation) which is elaborate and timeconsuming (up to one hour) and requires considerable skill and dedication. A possible limitation of the current study is the fact that only a single investigator created the postprocessed MR angiography images for comparison with catheter angiography. A study involving the use of multiple independent investigators would allow to assess the inter-observer variability of the specialized post-processing steps.

Only three patients, who showed no abnormality after performing MR and catheter angiography, were included. A larger true negative population would increase the clinical value of this comparative study. However, due to the applied selection criteria, that were based on clinical data and previous MRI findings, the number of patients that showed no abnormality was quite small.

\section{Future improvements}

A future possibility to characterize a SAVM more accurately would be the use of a second MR angiography examination in addition to the first MR angiography with the large field of view. In the second MR angiography examination a smaller field of view could focus on the vascular pathology detected in the first MR angiography examination and could reveal more details of the fistulous zone and/or arterial feeders.

Future developments that may allow us to improve the MR angiography examination are the use of high T1-relaxivity (e.g., blood pool) contrast agents. This could enable the more detailed depiction of the shunting zone, rather than merely localizing the arterial feeder. Whether the use of a blood pool agent itself is sufficient to improve the visualization of the small spinal cord vasculature remains uncertain as the conventional gadolinium contrast material remains within the intravascular compartment due to the blood-cordbarrier.

In theory imaging could improve when changing to a 3-Tesla magnet as it offers better signal-to-noise characteristics that can be used to increase spatial and temporal resolution and to better depict smaller vessels. However, yet there is in our opinion no obvious benefit in changing from a 1.5 to a 3-Tesla magnet. The major advantage of the 1.5-Tesla system is the ability to use a dedicated spine coil, which enables a sufficiently homogeneous field of view up to $500 \mathrm{~mm}$ and but is not (yet) available for our 3-Tesla MRI system. The large field of view helps to localize the variable origin of the feeders of the vascular abnormalities as well as the Adamkiewicz artery along the entire thoracolumbar-sacral spinal cord. Achieving such a large field of view is not possible with current 3-Tesla systems. Moreover, the stronger field 
inhomogeneities near the vertebral bodies seem to counteract the increase in signal-tonoise ratio. Therefore, a major technological challenge is to be taken to improve the 3-Tesla MRI system for spinal cord angiography.

In conclusion, we demonstrated that MR angiography is able to visualize both normal and pathologic arteries and veins of the spinal cord. The arterial feeder of a SDAVF can be accurately pin-pointed by MR angiography, but for SAVM only the predominant arterial feeder can be visualized, and subclassification, which is relevant for the choice of treatment, should still be based on catheter angiography. Spinal cord AV abnormalities can be reliably detected or excluded by MR angiography (i.e. $100 \%$ predictive value), which may focus burdensome catheter angiography procedures or even avoid them in cases of normal MR angiography. 


\section{REFERENCES}

1. Lasjaunias P. Spinal and spinal cord arteries and veins. In: Lasjaunias P, Berenstein, A, Ter Brugge K.G., ed. Surgical Neuroangiography. Berlin: Springer-Verlag; 2001:73-160.

2. Thron A. Vascular anatomy of the spine. In: Byrne J, ed. Interventional Neuroradiology. Oxford: Oxford University Press;2002:19-23.

3. Thron A. Clinical applications. In: Thron A, ed. Vascular anatomy of the spinal cord. Wien: SpringerVerlag;1988:65-105.

4. Forbes G, Nichols DA, Jack CR, Jr., et al. Complications of spinal cord arteriography: prospective assessment of risk for diagnostic procedures. Radiology 1988;169:479-84.

5. Krings $T$, Mull M, Gilsbach JM, Thron A. Spinal vascular malformations. Eur Radiol 2005;15:267-78.

6. Luetmer PH, Lane JI, Gilbertson JR, Bernstein MA, Huston J, 3rd, Atkinson JL. Preangiographic evaluation of spinal dural arteriovenous fistulas with elliptic centric contrast-enhanced MR Angiography and effect on radiation dose and volume of iodinated contrast material. AJNR Am J Neuroradiol 2005;26:711-8.

7. Pattany PM, Saraf-Lavi E, Bowen BC. MR angiography of the spine and spinal cord. Top Magn Reson Imaging 2003;14:444-60.

8. Mascalchi M, Quilici N, Ferrito $G$, et al. Identification of the feeding arteries of spinal vascular lesions via phase-contrast MR angiography with threedimensional acquisition and phase display. AINR Am I Ncuroradiol 1997;18:351-8.

9. Bowen BC, Fraser K, Kochan JP, Pattany PM, Green BA, Quencer RM. Spinal dural arteriovenous fistulas: evaluation with MR angiography. AJNR Am J Neuroratiol 1995;16:2029-43.

10. Binkert CA, Kollias SS, Valavanis A. Spinal cord vascular disease: characterization with fast threedimensional contrast-enhanced MR angiography. AJNR Am J Neuroradiol 1999;20:1785-93.
11. Farb RI, Kim JK, Willinsky RA, et al. Spinal dural arteriovenous fistula localization with a technique of first-pass gadolinium-enhanced MR angiography: initial experience. Radiology 2002;222:843-50.

12. Nijenhuis RJ, Leiner T, Cornips EM, et al. Spinal cord feeding arteries at MR angiography for thoracoscopic spinal surgery: feasibility study and implications for surgical approach. Radiology 2004;233:541-7.

13. Nijenhuis RJ, Mull M, Wilmink JT, Thron AK, Backes WH. MR angiography of the great anterior radiculomedullary artery (Adamkicwicz artery) validated by digital subtraction angiography. AINR Am I Neuroradiol 2006;27:1565-72.

14. Nijenhuis RJ, Gerretsen S, Leiner T, Jacobs MJ, van Engelshoven JM, Backes WH. Comparison of 0.5-M Gd-DTPA with 1.0-M gadobutrol for magnetic resonance angiography of the supplying arteries of the spinal cord in thoracoabdominal aortic aneurysm patients. J Magn Reson Imaging 2005;22:136-44. 


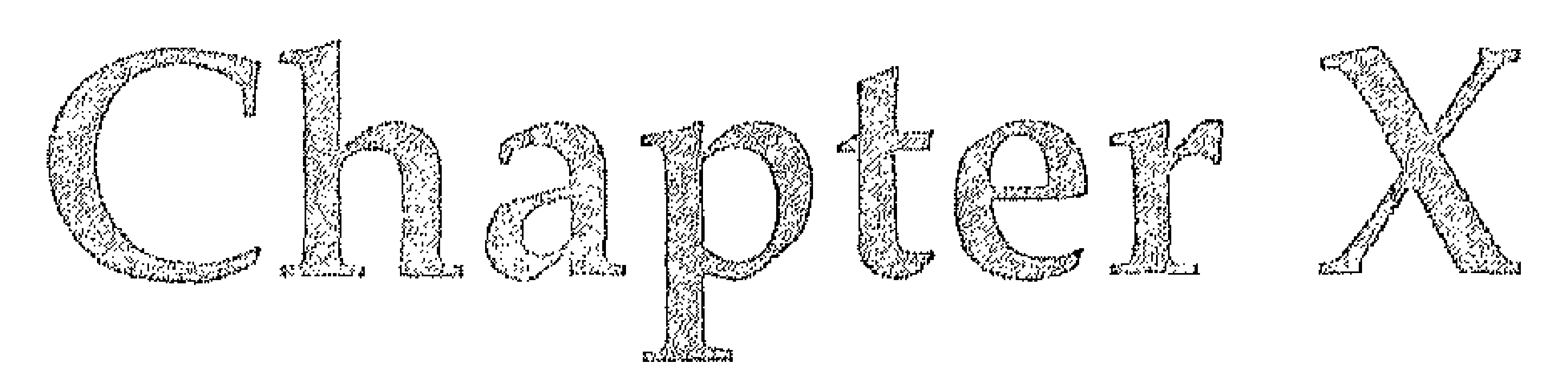

General discussion 
This thesis was set out to develop a noninvasive imaging technique that is capable of visualizing the blood supplying trajectories to the spinal cord in order to allow further reduction of ischemic damage of the spinal cord in TAAA repair. The inlet artery that is considered to be most important for the blood supply to the thoracolumbar spinal cord is the artery of Adamkiewicz. This artery and its supplying trajectories should be preserved when performing interventions that could potentially damage the spinal cord.

\section{$M R$ angiography of the spinal cord}

After Yamada et al ${ }^{1}$ in 2000 for the first time described the use of magnetic resonance (MR) angiography to image the Adamkiewicz artery in a non-invasive manner, it has become a promising alternative to invasive catheter angiography. The small vascular calibres, the complicated vascular anatomy, and the many vascular anatomic variations make spinal cord MR angiography rather difficult and challenging. This thesis describes the development of a MR angiography technique that is able to detect the Adamkiewicz artery in a (i) reliable, (ii) reproducible, and (iii) verifiable way. (i) Reliability of Adamkiewicz artery detection using MR angiography was proven by obtaining a $100 \%$ detection ratio in several studies performed in patients with and without vascular disease (chapter II, III, \& VIII). In addition, differentiation from the similarly shaped outlet vein (i.e., great anterior radiculomedullary vein) was realized, thus avoiding misinterpretation between the Adamkiewicz artery and outlet vein (chapter IV). (ii) Using a new contrast agent (Gadobutrol, $1.0 \mathrm{~mol} / \mathrm{L}$ ) it was shown that the Adamkiewicz artery could be localized at the same, i.e., reproducible, vertebral level as found by the MR angiography exam performed with the conventional MR contrast agent (gadopentetate dimeglumine, 0.5 $\mathrm{mol} / \mathrm{L}$ ) (chapter III). (iii) By subjecting patients suspected of having a vascular spinal cord abnormality to successive MR angiography and catheter angiography exams (i.e., standard of reference), the localization of the Adamkiewicz artery by MR angiography could be validated and showed excellent agreement with catheter angiography (chapter $V$ ). In addition, in a postmortem specimen of a TAAA patient the localization of the Adamkiewicz artery and outlet vein coincided with the preoperatively determined positions by MR angiography (chapter VI).

Apart from the Adamkiewicz artery, additional anterior radiculomedullary arteries may contribute to the supply of the anterior spinal artery. As additional anterior radiculomedullary arteries are by definition smaller than the Adamkiewicz artery, they usually display a lower signal intensity. Since the focus in the first two studies (chapter II \& III) was only on the first dynamic phase, which was thought to be predominantly arterial, additional vessels with less intensity observed in the first phase were classified as additional anterior radiculomedullary arteries. However, designating the additionally depicted vessels as arterial may be uncertain for several reasons. First of all, localization of the outlet vein was not performed. Secondly, MR angiography is a relatively slow technique and uses non-selective administration of a relatively large amount of contrast material, which both complicate separate visualization of arteries and veins. Finally, no validation study was performed in these first two studies. Therefore, it remains unclear whether these depicted additional vessels represented 
additional small arteries or the early, thus partial, enhancement of veins.

Comparison of computed tomography (CT) with MR angiography for their capability to localize the Adamkiewicz artery, revealed that MR angiography is far more sensitive (chapter VII). The average detection rate for MR angiography was found to be $97 \%$ compared to $71 \%$ for CT angiography. In contrast to $\mathrm{CT}$ angiography, MR Angiography was not affected by the patients' physical dimensions. In addition, only MR angiography was able to differentiate between the Adamkiewicz artery and the similarly shaped outlet vein by measuring the signal intensity time courses of these vessels in two subsequent dynamic phases. To obtain this important separation of the inlet artery from the outlet vein with $\mathrm{CT}$ angiography, would either require a second phase or a faster CT system, for which both vessels were visualized simultaneously. Increasing the number of phases with CT would, however, further increase the already high $X$-ray exposure. The option of using a faster CT system (i.e., larger craniocaudal coverage per time unit) will require a very accurate timing of the contrast arrival, as in CT angiography each slice is acquired only once and slice acquisition time for the faster systems is quite below 0.5 second. Although arteriovenous separation with CT angiography seems difficult to achieve, it would, at least from a logistical point of view, be beneficial to use CT angiography for the detection of the Adamkiewicz artery. The reason for this is that CT angiography is already applied in the preoperative work-up of TAAA patients to determine the extent of aortic pathology.

The lower detection ratio for the Adamkiewicz artery with CT (71\%) compared to $\mathrm{MR}$ angiography (97\%) obtained in our study is in contrast to the first head-to-head comparison study performed by Yoshioka et $\mathrm{al}^{2}$. In their study a somewhat better detection ratio was found for CT (80\%) compared to MR angiography $(67 \%)$ in 30 Japanese TAAA patients. Surprisingly, in a very recently published second head-to-head comparison by this group ${ }^{3}$, in a similar TAAA patient population, they obtained results comparable to our findings. Adamkiewicz artery detection ratio was $93 \%$ for MR angiography and $83 \%$ for CT angiography. The explanation provided for this remarkable improvement of MR angiography was the use of a new more sensitive spine coil and faster gradients. Interestingly, all studies, except for one ${ }^{4}$, performed in Japanese aortic aneurysm patients using $C T$ angiography showed higher detection ratios $(80-93 \%)^{2,3,5,6}$ compared to our ratio of $71 \%$. This discrepancy may be explained by differences in image quality caused by the exact implementation of the imaging techniques, and patient populations. Probably the main reason for the lower detection rate with CT angiography in our Caucasian patient group is that the physical dimensions of our TAAA patients are generally larger compared to Japanese. In other words, Caucasian patients are more corpulent, which prohibits a larger part of the emitted $X$-ray photons to transmit to the CT detector arrays. Therefore, MR angiography is in our opinion the preferred imaging modality in Caucasian TAAA patients.

When introducing spinal cord MR angiography in general clinical practice the major difficulty that likely will be encountered is that of high-quality image postprocessing. Localization and separation of the inlet artery and outlet vein of the spinal cord requires 
quite some experience in postprocessing techniques (curved multiplanar reformation and targeted maximum intensity projection) and knowledge of vascular anatomy of the spinal cord. Therefore, it is advised that devoted radio-technicians should be trained to perform the MR angiography acquisition and image postprocessing. Application without thorough knowledge of image postprocessing and vascular anatomy will likely result in decreased detection ratios that will diminish the clinical utility of the technique.

\section{Benefit for TAAA surgery}

The most important study of this thesis, which was conducted in 60 TAAA patients treated by open surgery, revealed that there is a significant relation between the location of the segmental artery supplying the Adamkiewicz artery relative to the aortic cross-clamp area and the intraoperative spinal cord function monitored by motor-evoked potentials (chapter VIII). More specifically, when the segmental supplier of the Adamkiewicz artery was outside the cross-clamped aortic area no changes in spinal cord function were observed. This means that MR angiography provides a negative predictive value of $100 \%$. This finding can be of use when determining aortic clamp positions and landing zones of an endovascular stent graft. On the other hand, when the segmental supplier was localized inside the cross-clamped aortic area, the positive predictive value of MR angiography for a decrease in spinal cord function was found to be only $32 \%$. This implied that spinal cord function in $68 \%$ of the cases was not crucially dependent on a single segmental supplier to the Adamkiewcz artery, but could rely on the combination of intraoperative protective measures (cooling, CSF drainage, and distal aortic perfusion) and collateral blood supply. However, collateral supply can be vulnerable especially in low blood pressure situations, as encountered in one case where postoperative paraplegia occurred after a period of low blood pressure. Preventive reattachment of the segmental supplier of the Adamkiewicz artery, even when spinal cord function is not dependent on this supply, can provide additional protection. This reattachment can be guided by preoperative MR angiography, similar to cases where spinal cord function is dependent on the segmental supply. The preoperative localization by MR angiography in our series helped the surgeon to selectively revascularize the segmental supplier and preserve spinal cord function in 12 out of 14 cases, in which the motor evoked potentials signalled (temporary) spinal cord dysfunction during aortic cross-clamping.

Permanent spinal cord dysfunction could unfortunately not be prevented in all patients. At present the incidence of paraplegia in our highly specialized center is $4 \%$ in over 300 procedures. With MR angiography as an additional protective measure the paraplegia rate was 5\% ( 3 out of 60 patients). To really assess whether MR angiography is able to reduce the incidence of postoperative paraplegia a comparable group of 200 to 300 TAAA patients has to be studied. Since our inclusion was limited to 60 patients, we cannot infer on whether preoperative MR angiography does or does not reduce the incidence of postoperative paraplegia. Moreover, even when MR angiography would add to the reduction of the paraplegia incidence, complete prevention of paraplegia seems not realistic yet as reattachment of a selective prosthetic graft to the segmental 
artery supplying the Adamkiewicz artery may take too much time and grafts may thrombose. Taken together, preoperative MR angiography now provides information that was not available before and can guide selective reattachment of the segmental supplier of the Adamkiewicz artery.

Outlook. Further development of MR angiography in preoperative TAAA patients should focus on the visualization of the collateral blood supplying trajectories (Figures $10.1 \&$ 10.2). More detailed preoperative information on the existence and trajectories of collaterals may allow improved risk estimation for the incidence of postoperative neurologic complications and planning of surgical strategies. Apart from improving preoperative imaging also interest should be directed to postoperative MR angiography in order to gain more insight in the development of the collateral circulation and how to stimulate such development to preserve spinal cord blood supply (Figure 10.3). Furthermore, postoperative imaging could also be used to evaluate the status of the prosthetic vascular graft of segmental arteries and their contribution to the spinal cord circulation.

In theory imaging could improve when changing to a 3-Tesla magnet as it offers better signal-to-noise characteristics that can be used to increase spatial and temporal resolution. However, there is in our opinion at present no obvious benefit in changing from a 1.5 to a 3 Tesla magnet. The major advantage of our 1.5Tesla system is the availability of a dedicated spine coil, which enables a sufficiently homogeneous field of view up to $500 \mathrm{~mm}$. This large field of view helps to localize the variable origin of the Adamkiewicz artery and its possible pelvic or cranial collateral supplying network. Achieving such a large field of view is not possible with current 3Tesla systems. Moreover, the stronger field inhomogeneities at 3-Tesla near the vertebral bodies seem to counteract the increase in signal-to-noise ratio. Therefore, a major technological challenge is to be taken to improve the 3-Tesla MRI system for spinal cord angiography. 

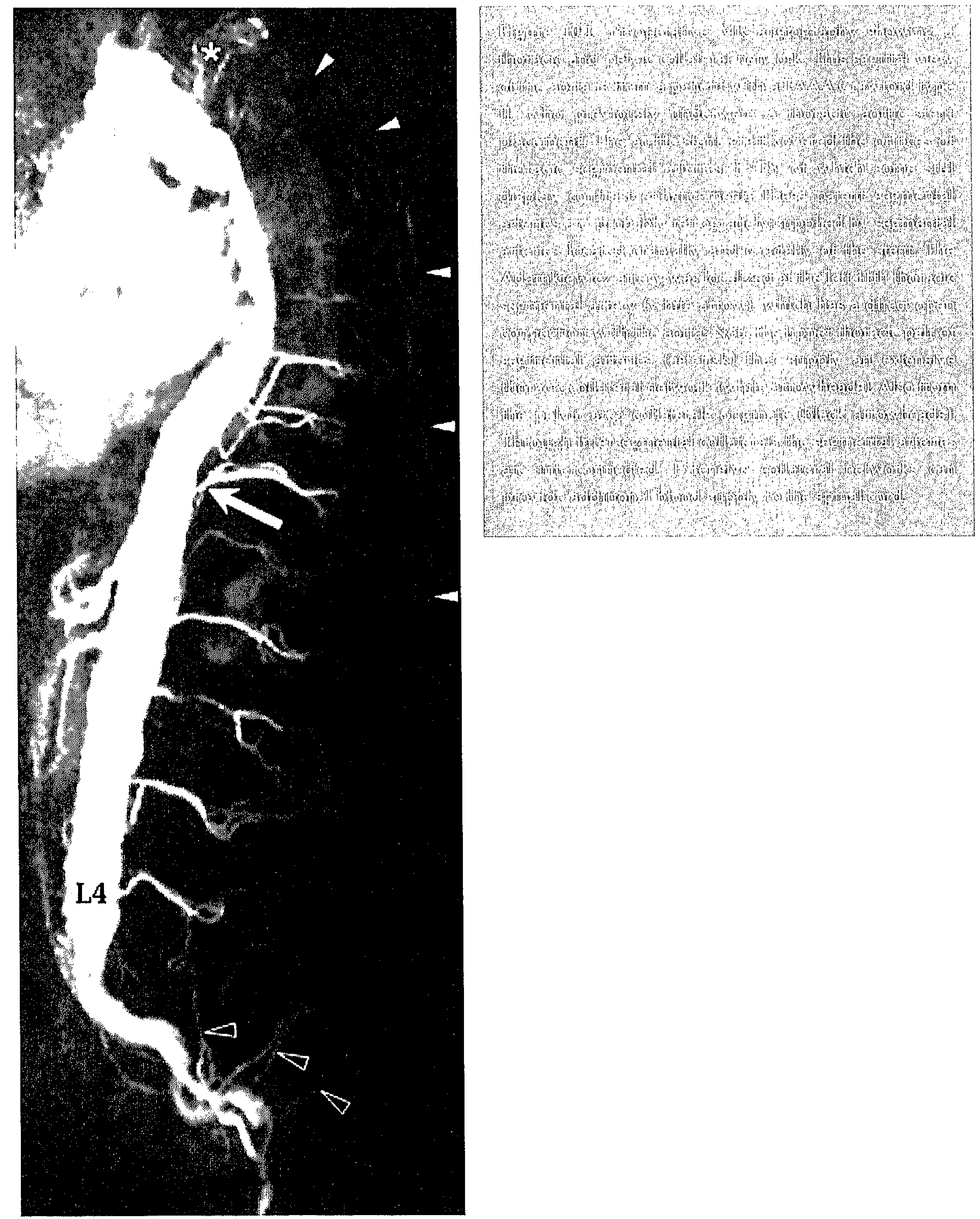

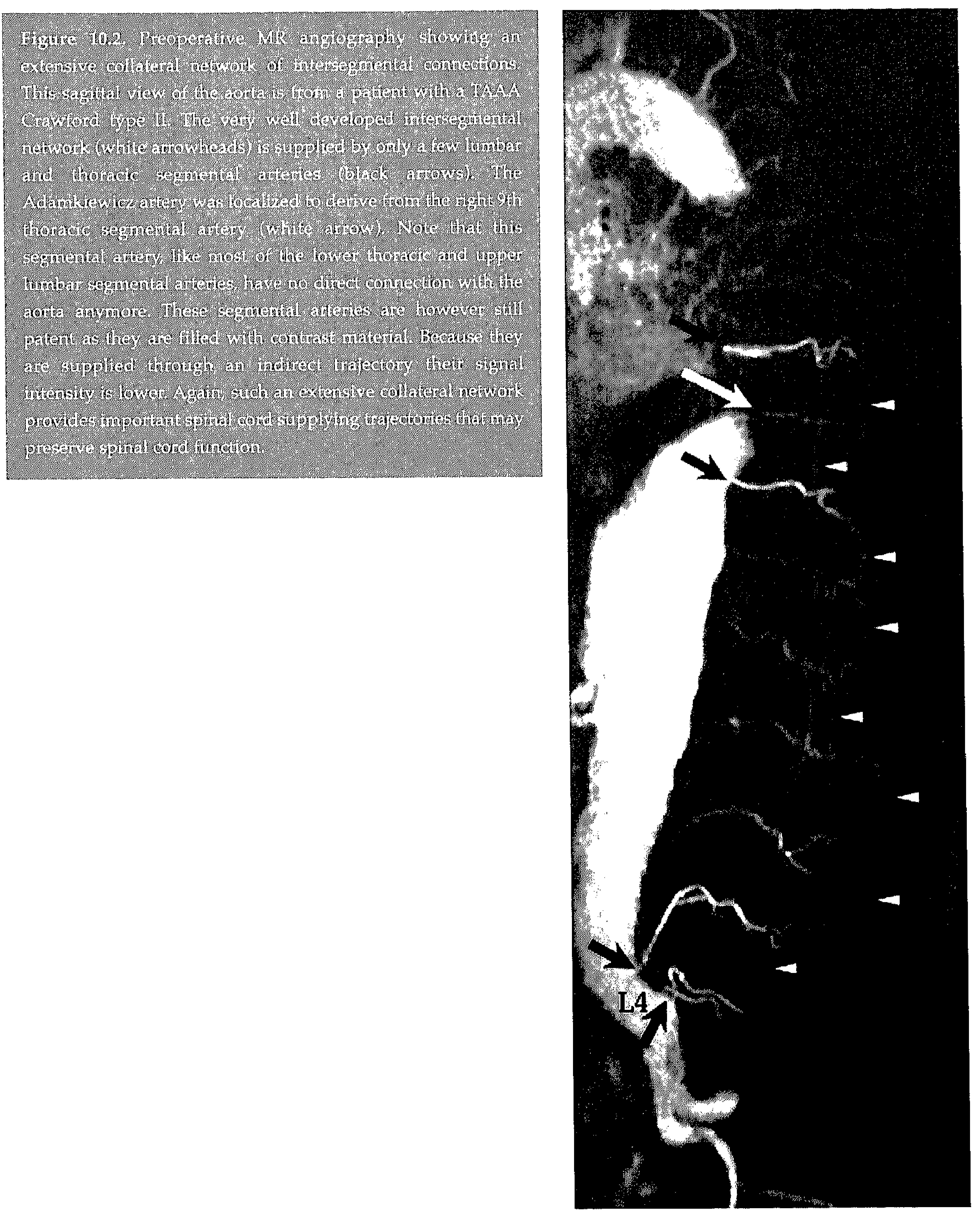
General discussion

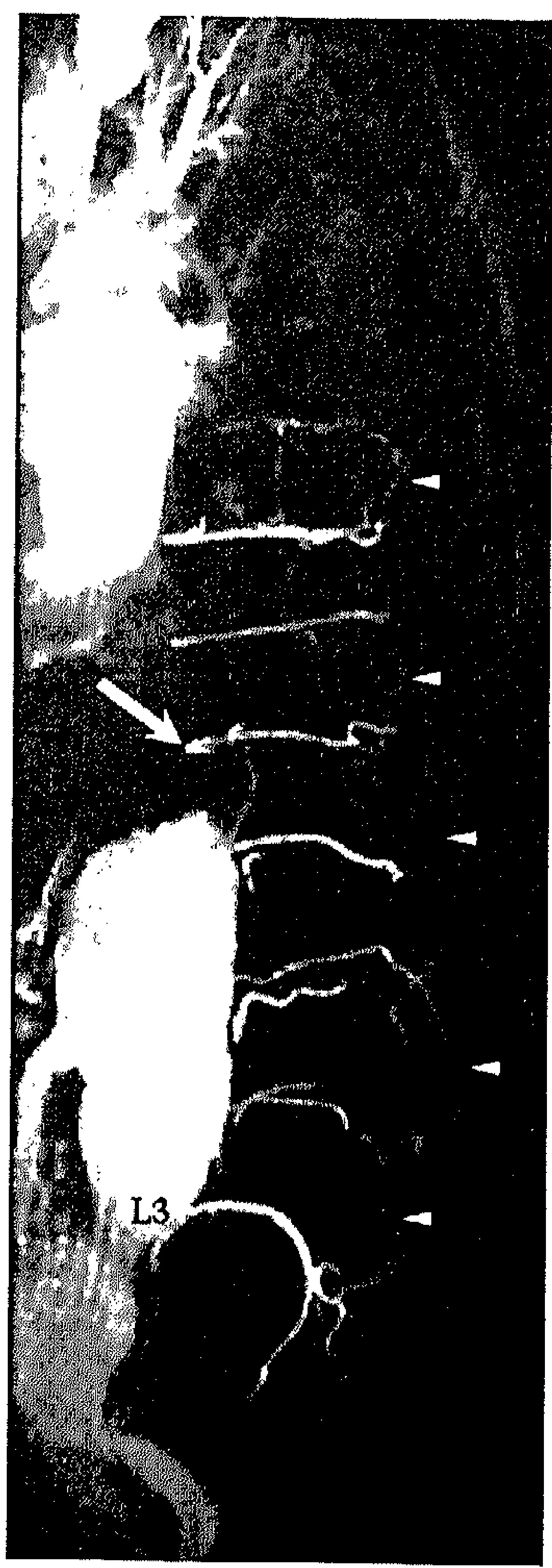

a

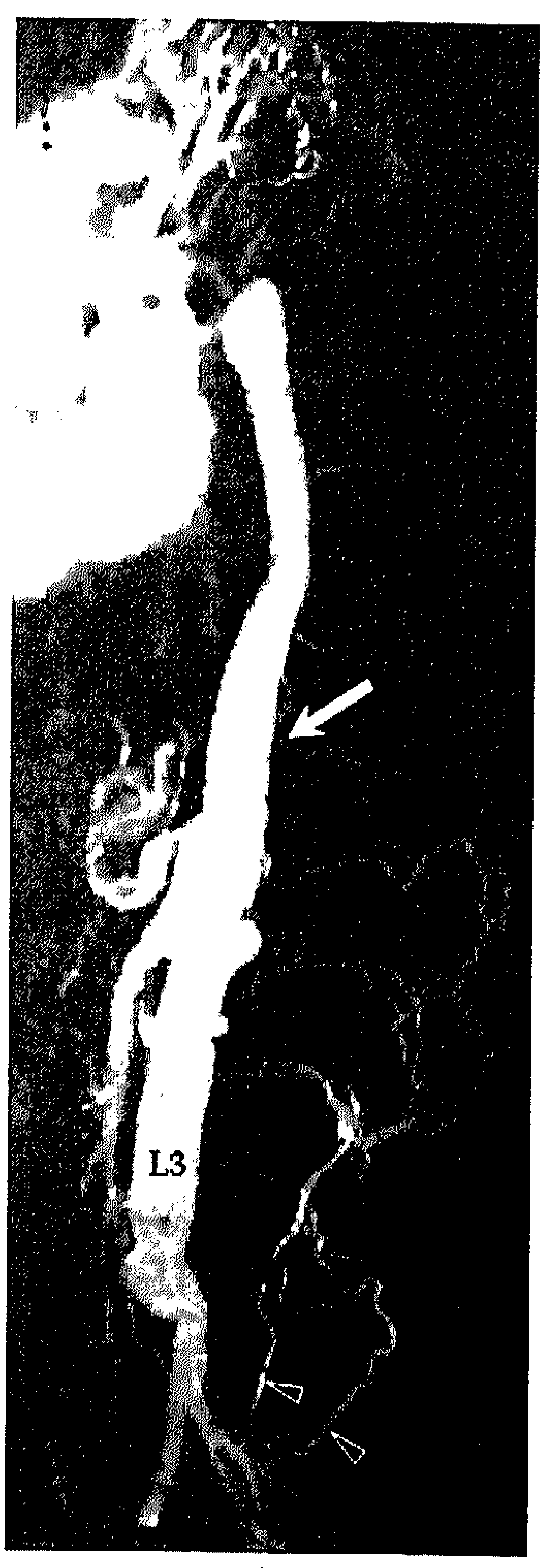

b

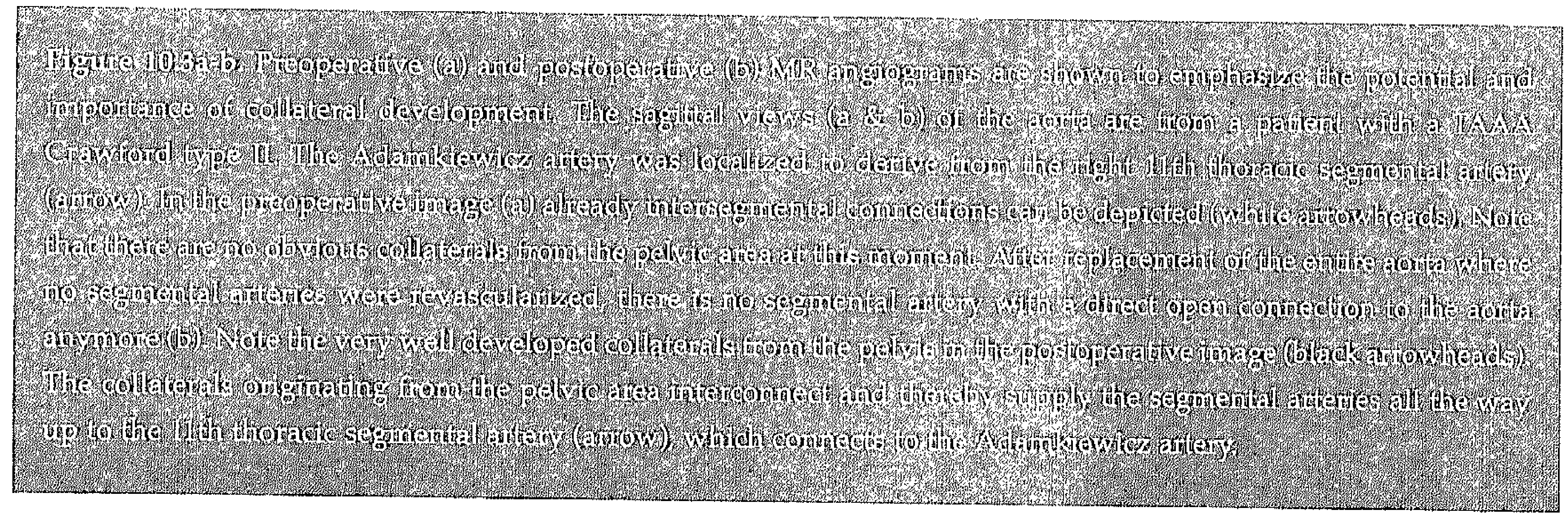


Benefit in vascular spinal cord pathology Another vascular pathology for which MR angiography is of interest concerns patients with suspected vascular abnormalities of the spinal cord (chapter IX). These can be divided into arteriovenous (AV) fistulas, $\mathrm{AV}$ malformations, and cavernomas. Since cavernomas are quite rare we focused on the other two more common vascular abnormalities. MR angiography proved, in a head-to-head comparative study with catheter angiography, to reliably localize the level of the fistula within one vertebral level. This guided the subsequent catheter angiography procedure in such a manner that only one catheter angiography exam was sufficient to confirm and localize the AV fistula. Moreover, unnecessary diagnostic catheter angiography procedures were prevented in patients, who appeared to have no vascular abnormalities. In contrast to $\mathrm{AV}$ fistulas, the attributive value of MR angiography to diagnose AV malformations appeared not that well defined as only the main arterial feeder, of possibly multiple feeders, could be localized. Definite classification, which is important for subsequent therapy, was in a number of cases not possible with MR angiography and still had to be based on catheter angiography. However, the preceding spinal cord MR angiography exam clearly localized the level of the $A V$ malformation and provided more insight into the spatial extensiveness of the vascular abnormality. In sum, MR angiography has become a clinically relevant non-invasive angiography technique that can diagnose and exclude vascular abnormalities of the spinal cord and should be applied before catheter angiography.

Outlook. In patients with arteriovenous malformations the major challenge is to discriminate and discern all individual feeders. This might be achieved by employing a two step approach in which the first exam will be used to localize the level and extent of the vascular abnormality and the second exam will be targeted to the specific (e.g., shunt zone) area using a higher spatial and temporal resolution. In this respect the second examination might improve on a 3-Tesla MR system.

\section{Conclusions}

The clinical introduction of spinal cord MR angiography has provided a reliable, reproducible, and patient-friendly imaging modality to visualize the spinal cord blood supply. The consistent detection of the Adamkiewicz artery has made MR angiography an attractive alternative to catheter angiography. As a clinical utility spinal cord MR angiography has shown to be a useful tool in the preoperative work-up of TAAA patients to localize the supplying segmental trajectories to the Adamkiewicz artery. In addition, MR angiography proved to be very helpful to diagnose patients suspected with arteriovenous abnormalities of the spinal cord. 


\section{REFERENCES}

1. Yamada N, Takamiya M, Kuribayashi S, Okita $Y$, Minatoya K, Tanaka R. MRA of the Adamkiewicz artery: a preoperative study for thoracic aortic aneurysm. J Comput Assist Tomogr 2000;24:362-8.

2. Yoshioka $K$, Niinuma $H$, Ohira $A$, et al. $M R$ angiography and $C T$ angiography of the artery of Adamkiewicz; noninvasive preoperative assessment of thoracoabdominal aortic aneurysm. Radiographics 2003;23:1215-25

3. Yoshioka K, Niinuma $H$, Ehara S, Nakajima T, Nakamura $M$, Kawazoe $K$. MR angiography and $C T$ angiography of the Artery of Adamkiewicz: State of the Art. Raliographics 2006;26 Suppl 1:\$63-73.

4. Kudo K, Terae S, Asano T, et al. Anterior spinal artery and artery of Adamkiewicz detected by using multidetector row CT. AJNR Am I Nenroradiol 2003;24:13-7.

5. Takase K, Sawamura Y, Igarashi $K$, et al. Demonstration of the artery of Adamkiewicz at multidetector row helical CT. Ratiology 2002;223:39-45.

6. Takase K, Akasaka J, Sawamura Y, et al. Preoperative MDCT evaluation of the artery of Adamkiewicz and its origin. / Comput Assist Tomogr 2006;30:716-22. 


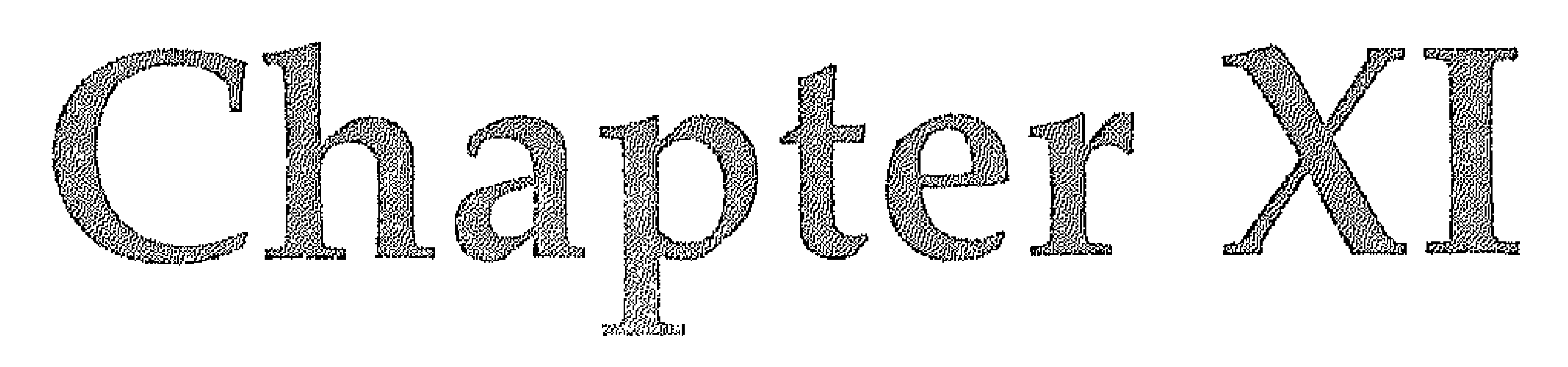

Summary and Conclusions 
Aneurysms of the thoracoabdominal aorta (TAAA) comprise a life-threatening disorder. When the aorta reaches a diameter of more than $6 \mathrm{~cm}$ surgery is indicated. During surgery the aorta is cross-clamped and the aneurysm is replaced by a prosthetic graft. One of the most feared and devastating events that can occur during and after surgery is paraplegia. The cause for this complication is temporary or permanent interruption of the blood supply to the spinal cord. To prevent paraplegia it is of absolute importance to preserve, and when needed to restore, the blood supply to the spinal cord. The largest and therefore considered the most important supplier of the spinal cord is the artery of Adamkiewicz. Until very recently catheter angiography was the only imaging modality capable of visualizing the Adamkiewicz artery. However, the use of catheter angiography in TAAA patients has two major disadvantages. First of all, it is not possible to localize the Adamkiewicz artery in all TAAA patients (detection rate between 43 and $86 \%$ ). Secondly, this invasive imaging technique may induce paraplegia in itself. Due to recent technical improvements it has become possible to visualize blood vessels of the spinal cord with non-invasive imaging modalities such as $M R$ angiography. The aims of this thesis were to develop a reliable MR angiography technique that is able to consistently localize the Adamkiewicz artery and its segmental supplier, to validate this MR angiography technique, compare it with $\mathrm{CT}$ angiography, and finally to assess the clinical utility of MR angiography.

In chapter II, we have investigated if the MR angiography technique developed, was capable to visualize and localize the Adamkiewicz artery and its segmental supplier in 8 patients with a herniated thoracic intervertebral disc. In these non-vascular compromised patients preoperative localization can be of importance, as during (thoracoscopic) surgery segmental arteries may be damaged or deliberately sacrificed to prevent bleeding. If this were to concern the segmental supplier of the Adamkiewicz artery this could lead to ischemia of the spinal cord and thus paraplegia. MR angiography was found to be capable of localizing the Adamkiewicz artery and its segmental supplier in all 8 patients. Preoperative knowledge of the segmental supplier level led in 3 cases to a change in the side of surgical approach, to avoid any damage to this segmental supplier. In none of the 8 operated patients postoperative paraplegia occurred.

In chapter III, two different contrast agents were explored to optimize MR angiography image quality and to test the reproducibility in localizing the artery of Adamkiewicz in TAAA patients. The use of the new contrast agent (Gadobutrol 1.0-M) did not improve image quality. Nevertheless, it was confirmed that MR angiography could reproducibly localize the Adamkiewicz artery and its segmental supplier.

In chapter IV, we have tested a newly developed MR angiography technique that aimed to improve the differentiation between the inlet artery (i.e., the Adamkiewicz artery) and the similarly shaped outlet vein (i.e., great anterior radiculomedullary vein). The reason for conducting this study was the observation that the outlet vein was already enhanced in the first dynamic phase in many of the TAAA patients. This new MR angiography technique allowed faster scanning, which enabled complete separation between the inlet artery and outlet vein based on time differences in 
contrast arrival. A disadvantage of this new technique was that the field of view, within the required time resolution, was limited to the spinal cord itself. Therefore, the entire trajectory of the supplying segmental artery from the aorta to the spinal cord could not be visualized, which is, however, of importance for clinical use. Nevertheless, this was the first MR angiography technique described that showed complete separation of inlet artery and outlet vein based on time difference of contrast arrival.

In chapter $V$, the first of two validation studies is described. In this first study, MR angiography based localization of the Adamkiewicz artery was compared with catheter angiography (i.e., standard of reference) in patients with a suspected vascular malformation of the spinal cord. MR angiography could correctly localize the level and side of the Adamkiewicz artery in 14 out of 15 patients. In contrast to catheter angiography, MR angiography could localize the outlet vein of the spinal cord. Convincing separation between the inlet artery and outlet vein could be accomplished using the twophase MR angiography approach and analysis of signal intensity courses of these two vessels.

In chapter VI, the second validation study is described. Here the spinal cord of a postmortem TAAA patient was prepared to determine the course and origin of the Adamkiewicz artery and the outlet vein for comparison with preoperative MR angiography of this deceased patient. The twophase MR angiography could localize the Adamkiewicz artery and separate it from the outlet vein. Both localization and configuration of these two spinal cord vessels as visualized by MR angiography were in complete agreement with the spinal cord specimen.
In chapter VII, computed tomography (CT) and $\mathrm{MR}$ angiography were compared to investigate which of these two modalities is most suited for localizing the Adamkiewicz artery in TAAA patients. Apart from localizing the Adamkiewicz artery the image quality was also assessed in 39 patients. Using MR angiography it was found that two observers were able to localize the Adamkiewicz artery on average in $97 \%$ of the cases compared to $71 \%$ with CT angiography. Overall image quality was also judged to be better for MR angiography. The most important explanation why MR angiography performed better than $\mathrm{CT}$ angiography was that a higher contrast-tonoise ratio for the Adamkiewicz artery was accomplished using MR angiography. The contrast-to-noise ratio in $\mathrm{CT}$ angiography was moreover found to decrease with increasing abdominal diameters, while for MR angiography the contrast-to-noise ratio was independent of abdominal diameter. Since TAAA patients are often corpulent, MR angiography is the preferred imaging modality for the localization of the Adamkiewicz artery in TAAA patients.

In chapter VIII, we have assessed the clinical value of preoperative MR angiography in combination with intraoperative neuromonitoring for the localization of the crucial spinal cord blood supplying trajectory. It was found that this trajectory could either be direct or indirect. Usually the blood supplying trajectory runs from the aorta via the segmental artery (at the level of the Adamkiewicz artery), to the Adamkiewicz artery and finally to the anterior spinal artery and spinal cord. Segmental arteries can become partially or completely occluded during the course of aortic aneurysmal disease. We found in TAAA patients with 
atherosclerotic vascular disease, that of the 30 segmental arteries that usually exist between vertebral levels T3 and L5, on average only 9 segmental arteries had an open direct connection with the aorta. In patients with an aortic dissection we found that on average 18 segmental arteries were still open. In cases where the segmental artery at the level of the Adamkiewicz artery was partially or completely occluded, MR angiography was able to visualize an extra-anatomical collateral trajectory that derived from an open segmental artery which originated from a higher or lower vertebral level. It is important to note that in all patients a left heart bypass was used. This provides arterial perfusion caudal to most distally placed aortic clamp. In this manner only the aortic cross-clamped area is not perfused, in contrast to the aortic areas above and below the aortic clamps. When the preoperatively visualized blood supplying trajectory to the spinal cord was not excluded by aortic cross-clamping from aortic circulation, the spinal cord function was unaffected. This implied a negative-predictive value of $100 \%$. On the other hand, when the blood supplying trajectories were excluded from aortic circulation by the aortic clamps, spinal cord function decreased only in $32 \%$ of the cases. This meant that in $68 \%$ of the cases spinal cord function was not dependent on the preoperatively visualized trajectory. These patients must rely on additional collateral trajectories that maintain spinal cord function. The Adamkiewicz artery could be preoperatively localized in $100 \%$ of the patients by MR angiography. However, localization of the Adamkiewicz artery only is apparently not sufficient and collateral supplying trajectories have to be depicted as well. Visualization of these additional trajectories forms the new goal of preoperative MR angiography.

In chapter IX, the clinical utility of MR angiography in patients with suspicion of a vascular spinal cord abnormality is investigated. Localization of the arterial feeders of a vascular abnormality, in advance to catheter angiography (i.e., standard of reference), could reduce the amount of injected contrast material as well as the exposure time to ionizing radiation. In addition, MR angiography may prevent unnecessary invasive catheter angiography in patients without any vascular abnormality. We obtained no false positive or negative findings with MR angiography in 34 patients. The exact vertebral level of an arteriovenous fistula, which is the most common vascular abnormality of the spinal cord, could be localized within one vertebral level in all patients. Considering arteriovenous malformations, MR angiography is at the moment only capable to localize the largest arterial feeder. Catheter angiography is therefore still required to localize possible additional arterial feeders and to classify different subtypes of arteriovenous malformations, which is mandatory for therapy choice. The most important benefit of $M R$ angiography for this patient population is that it allows non-invasive assessment of the presence of absence of a vascular spinal cord abnormality. This enables a more targeted catheter angiography on the one hand or can prevent such unnecessary invasive diagnostic procedures on the other hand. 


\section{CONCLUSIONS}

1. The MR angiography technique developed is able to consistently and reproducibly visualize and localize the Adamkiewicz artery and its segmental supplier.

2. Differentiation between the Adamkiewicz artery and the outlet vein (i.e., great anterior radiculomedullary vein), which are often simultaneously visualized in the first dynamic phase, is possible by using a two-phase MR angiography approach and signal intensity analysis of these two vessels as a function of time.

3. Complete separation of the Adamkiewicz artery and outlet vein, based on temporal differences in contrast enhancement, can be accomplished with fast dynamic MR angiography.

4. Validation studies using catheter angiography and a postmortem specimen show that MR angiography correctly localizes the Adamkiewicz artery and outlet vein.

5. In TAAA patients MR angiography achieves a higher detection ratio for localizing the Adamkiewicz artery as compared to $\mathrm{CT}$ angiography and therefore is the preferred imaging modality for this indication.

6. When the segmental supplier of the Adamkiewicz artery is not excluded from the direct aortic circulation, and thus is located outside the cross-clamped aortic area, the predictive value of MR angiography for an (unaffected) stable intraoperative spinal cord function is $100 \%$.

7. Localization of the Adamkiewicz artery and its segmental supplier are not sufficient to predict intraoperative spinal cord dysfunction in all TAAA patients. Depiction of collateral supplying trajectories is mandatory/necessary to achieve a better risk estimation.

8. The presence and localization of spinal dural arteriovenous fistula can accurately be diagnosed or excluded by MR angiography. This enables more targeted catheter angiography and can prevent such unnecessary invasive diagnostic procedures.

9. MR angiography is capable to localize the largest arterial feeder of an arteriovenous malformation. However, catheter angiography is still required for localizing additional arterial feeders and classification in the different subtypes, which is important for the choice of therapy. 
Nederlandse samenvatting 
Een aneurysma (bloedvatverwijding) van de thoracale en abdominale aorta (TAAA) is een levensbedreigende aandoening. Indien deze slagader een diameter bereikt van meer dan $5 \mathrm{~cm}$ vormt dit een indicatie voor een operatie. Bij deze operatie wordt de aorta afgeklemd en het aneurysmatische deel van de aorta vervangen door een vaatprothese. Een van de meest gevreesde complicaties die kan optreden door de operatie is het ontstaan van paraplegie (dwarslaesie). De oorzaak hiervan is dat de bloedvoorziening naar het ruggenmerg, die direct uit de aorta afkomstig is, gedurende de operatie kan worden onderbroken om de vaatprothese te kunnen inhechten. Om paraplegie te voorkomen is het van belang de bloedtoevoer tijdens de operatie te waarborgen en zonodig te herstellen. De grootste en daarom beschouwd als de belangrijkste voeder van het ruggenmerg is de arterie van Adamkiewicz. Tot op heden was het alleen met invasieve katheter angiografie mogelijk om de Adamkiewicz arterie te lokaliseren. Het gebruik van deze techniek in TAAA patiënten heeft echter twee grote nadelen. Ten eerste is katheter angiografie slechts in staat de arterie van Adamkiewicz in een beperkt aantal TAAA patiënten te lokaliseren (43-86\%) en ten tweede kan deze invasieve techniek zelf paraplegie induceren. Daarom wordt preoperatieve beeldvorming met katheter angiografie doorgaans niet toegepast in TAAA patiënten. Recente technische ontwikkelingen hebben het mogelijk gemaakt dat de bloedvaten van het ruggenmerg kunnen worden gevisualiseerd met behulp van niet-invasieve beeldvormende modaliteiten, zoals MR en CT angiografie. Het doel van dit proefschrift was een betrouwbare MR angiografie techniek te ontwikkelen, die in staat zou moeten zijn de arterie van Adamkiewicz en zijn voedende segmentaal arterie consistent te kunnen lokaliseren. Voorts is de ontwikkelde MR angiografie techniek gevalideerd, vergeleken met CT angiografie en op klinische waarde getoetst.

In hoofdstuk II, is bepaald of de ontwikkelde MR angiografie techniek in staat is de arterie van Adamkiewicz en zijn voedende segmentaal arterie te visualiseren en te lokaliseren in patiënten met een thoracale intervertebrale hernia. Voor deze niet vasculair belaste patiënten kan preoperatieve lokalisatie van belang zijn, omdat tijdens de (thoracoscopische) operatie het risico bestaat dat segmentaal arteriën beschadigd raken of bewust opgeofferd worden om bloedingen te voorkomen. Indien dit de segmentale arterie betreft die de arterie van Adamkiewicz voedt, zou dit kunnen leiden tot het optreden van ruggenmerg ischemie en dus paraplegie. MR angiografie bleek in staat de arterie van Adamkiewicz en de voedende segmentaal arterie in alle 8 patiënten te kunnen lokaliseren. Dit leidde ertoe dat de operatieve benadering in een drietal gevallen veranderde van zijde, om de voedende segmentaal arterie van de Adamkiewicz te vermijden. In geen van de 8 geopereerde patiënten trad (postoperatieve) paraplegie op.

In hoofdstuk III, worden twee verschillende contrastmiddelen vergeleken met als doel de beeldkwaliteit van MR angiografie te optimaliseren en de reproduceerbaarheid te testen voor het lokaliseren van de arterie van Adamkiewicz in TAAA patiënten. Het gebruik van het nieuwe contrastmiddel (Gadobutrol, 1.0-M) leidde niet tot een verbeterde beeldkwaliteit. Deze studie bevestigde echter wel dat MR angiografie in staat is reproduceerbaar de arterie van Adamkiewicz en de voedende 
segmentaal arterie te lokaliseren.

In hoofdstuk IV, wordt een nieuwe MR angiografie methode onderzocht om de differentiatie tussen de arterie van Adamkiewicz en de grote anterieure drainerende ruggenmergsvene te verbeteren. De aanleiding voor deze studie was de observatie dat in veel TAAA patiënten de grote anterieure drainerende ruggenmergsvene reeds is aangekleurd in de eerste dynamische fase. Met deze nieuwe MR angiografie techniek kon sneller worden gescand waardoor separatie tussen arterie en vene mogelijk bleek op basis van tijdsverschil in contrast aankleuring. Een nadeel van deze techniek was dat het gebied dat kon worden gescand, waarbij de minimaal benodigde tijdsresolutie niet werd overschreden, beperkt bleef tot alleen het ruggenmerg zelf. Het traject van de voedende segmentaal arterie vanaf de aorta naar de Adamkiewicz arterie kon niet worden gevisualiseerd, hetgeen echter wel van belang is wanneer men deze techniek in de klinische praktijk wil gaan gebruiken. Desondanks was dit de eerste MR angiografie techniek die deze manier van temporele differentiatie bewerkstelligde voor de bloedvaten van het ruggenmerg.

In hoofdstuk $V$, wordt de eerste van twee validatie studies beschreven. In deze studie is de toepassing van MR angiografie voor de lokalisatie van de Adamkiewicz arterie gevalideerd met de gouden standaard katheter angiografie in patiënten met een mogelijke vasculaire afwijking van het ruggenmerg. MR angiografie bleek de arterie van Adamkiewicz correct te kunnen lokaliseren in 14 van de 15 patiënten. Daarnaast was MR angiografie ook in staat de grote anterieure drainerende ruggenmergsvene te detecteren. Met katheter angiografie daaren- tegen kon deze vene in geen enkele patiënt worden gevisualiseerd. Door de acquisitie van twee direct opeenvolgende dynamische fasen en signaal intensiteit analyse van de arterie en vene was het mogelijk deze vaten betrouwbaar van elkaar te onderscheiden.

In hoofdstuk VI, wordt de tweede validatie studie beschreven. In deze studie is het ruggenmerg van een geopereerde en helaas overleden TAAA patiënt vrij geprepareerd om het verloop van bloedvaten te vergelijken met $M R$ angiografie. De preoperatieve twee-fasen MR angiografie kon de arterie van Adamkiewicz lokaliseren en onderscheiden van de grote anterieure drainerende ruggenmergsvene. Zowel de lokalisatie als configuratie van deze twee ruggenmergsvaten bleken volledig overeen te komen met het vrij geprepareerde ruggenmerg.

In hoofdstuk VII, worden Computer Tomografische (CT) angiografie en MR angiografie met elkaar vergeleken om te bepalen welke van de twee beeldvormende modaliteiten het meest geschikt is voor de lokalisatie van de Adamkiewicz arterie in TAAA patiënten. Naast lokalisatie werd ook de beeldkwaliteit vergeleken. Uiteindelijk bleek dat twee observatoren de arterie van Adamkiewicz gemiddeld in $97 \%$ van 39 geïncludeerde patiënten konden lokaliseren met MR angiografie en slechts in $71 \%$ met CT angiografie. De algehele beeldkwaliteit werd eveneens beter beoordeeld voor MR angiografie. De belangrijkste verklaring waarom MR angiografie betere resultaten gaf was dat een gunstigere contrast ruis verhouding werd bereikt voor de Adamkiewicz artery ten opzichte van zijn directe omgeving. Verder bleek de contrast ruis verhouding voor $\mathrm{CT}$ angiografie af te nemen met toenemende buikomvang. Voor MR angiografie bleek een 
toename in buikomvang geen invloed te hebben op de contrast ruis verhouding. Aangezien TAAA patiënten vaak corpulent zijn, is MR angiografie de meest geschikte beeldvormende modaliteit voor de visualisatie van de Adamkiewicz in TAAA patiënten.

In hoofdstuk VIII, wordt de klinische waarde van preoperatieve MR angiografie onderzocht voor het lokaliseren van het cruciale bloedtoevoerende traject naar het ruggenmerg in combinatie met intraoperatieve neuromonitoring in de vorm van motor evoked potentials (MEPs). De bloedtoevoer naar het ruggenmerg bleek direct en indirect te kunnen verlopen. Normaliter verloopt het traject direct vanuit de aorta via de segmentaal arterie, op het niveau van de Adamkiewicz arterie, naar de Adamkiewicz arterie en uiteindelijk naar de arterie spinalis anterior en het ruggenmerg. Segmentaal arteriën kunnen echter in de loop van het ziekteproces van de aorta partieel of volledig geoccludeerd raken. In TAAA patiënten met atherosclerotisch vaatlijden bleek dat er van de 30 segmentaal arteriën, die normaal gesproken aanwezig zijn tussen het wervelniveau T3 en L5, gemiddeld nog maar 9 waren die een open (directe) verbinding met de aorta hadden. In patiënten met een dissectie daarentegen werden gemiddeld 18 open segmentaal arteriën gevonden. In die patiënten, waarbij de segmentaal arterie op het niveau van de Adamkiewicz arterie partieel of volledig was geoccludeerd, kon MR angiografie een extra-anatomisch traject visualiseren waarbij de Adamkiewicz arterie via een hoger of lager gelegen open segmentaal en intersegmentale connecties werd voorzien van bloed. Wanneer het met MR angiografie preoperatief gelokaliseerde bloedtoevoerende traject niet werd uitgeklemd bleef de ruggenmergsfunctie intact. Dit betekende dat de negatief voorspellende waarde $100 \%$ was. Anderzijds wanneer de bloedtoevoerende route wel werd afgesloten door het afklemmen van een deel van de aorta, daalde de ruggenmergsfunctie in $32 \%$ van de patiënten, hetgeen betekende dat de ruggenmergsfunctie in $68 \%$ van de patiënten niet afhankelijk was van het preoperatief gelokaliseerde traject. In deze patiënten moest er dus een additioneel (collateraal) traject bestaan dat de ruggenmergsfunctie in stand hield. MR angiografie bleek in 100\% van de patiënten in staat de Adamkiewicz arterie te lokaliseren. Kennelijk is het afbeelden van de Adamkiewicz arterie op zich dus niet voldoende en dient ook het toevoerende collaterale traject bepaald te worden. Visualisatie van dit additionele traject vormt het nieuwe speerpunt in preoperatieve MR angiografie.

In hoofdstuk IX, wordt de klinische toegevoegde waarde van MR angiografie voor patiënten met een verdenking op een vasculaire afwijking van het ruggenmerg beschreven. Lokalisatie van de arteriële voeders naar de vasculaire afwijking voorafgaand aan de gouden standaard, katheter angiografie, zou potentieel een reductie in de hoeveelheid gebruikt contrastmiddel en blootstelling aan ioniserende straling kunnen betekenen. Daarnaast zouden mogelijk ook onnodige diagnostische katheter angiografische onderzoeken kunnen worden voorkomen. Voor de detectie van vasculaire ruggenmerg afwijkingen werden in geen van 34 patiënten fout negatieve of fout positieve resultaten verkregen met MR angiografie. Het exacte wervelniveau van een arterioveneuze fistel, welke verreweg de meest voorkomende 
vasculaire afwijking is van het ruggenmerg, kon tot op een nauwkeurigheid van één wervelniveau in alle patiënten worden gelokaliseerd. Voor arterioveneuze malformaties is MR angiografie op dit moment alleen in staat de grootste arteriële voeder te detecteren. Voor de lokalisatie van additionele voeders en subclassificatie van de verschillende typen arterioveneuze malformaties, wat van belang is voor de therapiekeuze, is nog steeds katheter angiografie noodzakelijk. Het voordeel van MR angiografie is dat nu op een non-invasieve wijze de aanwezigheid van een vasculaire laesie kan worden aangetoond of worden uitgesloten. Hierdoor kan de katheter angiografie meer gericht worden uitgevoerd en worden onnodige invasieve diagnostische procedures voorkomen. 


\section{CONCLUSIES}

1. De ontwikkelde MR angiografie techniek is in staat consistent en reproduceerbaar de arterie van Adamkiewicz en zijn voedende segmentaal arterie te visualiseren en te lokaliseren.

2. Differentiatie tussen de arterie van Adamkiewicz en de grote anterieure drainerende ruggenmergsvene, welke vaak tegelijkertijd worden afgebeeld in de eerste fase, is mogelijk door gebruik te maken van twee-fasen MR angiografie en signaal intensiteit analyse van deze twee vaten als functie van de tijd.

3. Volledige separatie van de Adamkiewicz arterie en de grote anterieure drainerende ruggenmergsvene kan worden bereikt met snelle dynamische MR angiografie op basis van tijdsverschil in aankleuring van deze twee vaten.

4. Validatie studies verricht met katheter angiografie en een postmortem specimen tonen aan dat MR angiografie correct de arterie van Adamkiewicz en de grote anterieure drainerende ruggenmergsvene kan lokaliseren.

5. In TAAA patiënten heeft MR angiografie een hogere detectie ratio voor het lokaliseren van de arterie van Adamkiewicz dan CT angiografie en is daarom de te prefereren beeldvormende modaliteit voor deze indicatie.

6. Wanneer de voedende segmentaal arterie van de Adamkiewicz arterie tijdens de operatie niet wordt afgesloten van de directe circulatie uit de aorta, en zich dus buiten de aortale klemposities bevindt, is de voorspellende waarde van MR angiografie voor een stabiele intraoperatieve ruggenmergsfunctie $100 \%$.

7. Lokalisatie van de Adamkiewicz arterie en zijn voedende segmentaal arterie zijn niet toereikend voor het voorspellen van een daling van de intra-operatieve ruggenmergsfunctie in alle TAAA patiënten. Visualisatie van collaterale bloedvoorzienende trajecten lijkt nodig voor een betere risico inschatting van de kans op ruggenmergsfunctie dalingen.

8. Arterioveneuze fistels kunnen accuraat worden gelokaliseerd of uitgesloten met $M R$ angiografie. Hierdoor kan de katheter angiografie meer gericht en dus sneller worden uitgevoerd en worden onnodige invasieve diagnostische procedures voorkomen.

9. MR angiografie kan de grootste voeder van een arterioveneuze malformatie lokaliseren. Katheter angiografie is echter nog steeds nodig voor de lokalisatie van additionele voeders en subclassificatie van de verschillende typen arterioveneuze malformaties, hetgeen van belang is voor de therapiekeuze. 
Acknowledgements / Dankwoord 
Van promoveren wordt wel gezegd dat dit een investering is in jezelf. $\mathrm{Nu}$ aan het eind gekomen van mijn promotieperiode kan ik inderdaad zeggen dat ik heel veel heb geleerd, veel nieuwe contacten heb kunnen leggen maar vooral ook heel veel mooie herinneringen heb overgehouden aan deze periode. Echter de totstandkoming van dit geheel was niet mogelijk geweest zonder de bijdrage en investering van vele personen aan zowel de wetenschappelijke inhoud als de ontwikkeling van mijn eigen persoon. Ik heb het genoegen gehad te mogen samenwerken met veel inspirerende personen. Daarom wil ik graag mijn dank uit spreken naar al deze personen. Een aantal van hen wil ik graag persoonlijk bedanken. Allereerst wil ik de patiënten bedanken die hebben deelgenomen aan de studies beschreven in dit proefschrift.

Prof. dr. Michael Jacobs, mijn chirurgische promotor. Zonder uw vraag naar de functionele bloedvoorziening van het ruggenmerg zou dit hele proefschrift niet zijn opgezet. Uw enthousiasme, bekwaamheid en bevlieging in de behandeling van thoracoabdominale aorta aneurysmata is voor mij een grote stimulans geweest en zal dat waarschijnlijk ook blijven. De trip naar Philadelphia waar wij werk en hobby goed hebben kunnen combineren was fantastisch en onvergetelijk. Professor Jacobs, ik hoop dat wij in de toekomst kunnen blijven samenwerken in deze interessante 'tak van sport'.

Prof. dr. Jos van Engelshoven, mijn radiologische promotor. De atmosfeer die $\mathrm{u}$ creëert voor onderzoekers is ongekend en zeer stimulerend. Vanaf het begin van dit project heb ik in het midden gelaten welk specialisme ik wilde gaan uitoefenen. Naarmate het einde van mijn promotie naderde ben ik bezweken voor de chirurgie. Desondanks zal mijn interesse in de radiologie blijven bestaan. Bedankt voor de mogelijkheden die $u$ mij heeft gegeven.

Dr. ir. Walter Backes, mijn co-promotor. Walter, misschien dat je na mij nooit meer een arts wilt begeleiden, omdat dit toch een soort ongeleide projectielen zijn (althans mijn persoon). Ik wil je bedanken voor je geduld en alles wat je mij hebt geleerd. Jouw bewonderenswaardige drang naar perfectie, hetgeen resulteerde in altijd ontzettende rood gecorrigeerde stukken, hoezeer ik ook mijn best deed, hebben uiteindelijk wel geleid tot het feit dat alle artikelen van dit proefschrift zijn geaccepteerd. Ik hoop dat we deze reeks samen kunnen blijven uitbreiden in de toekomst.

Prof. dr. Jan Wilmink, zonder uw PRactiviteiten en contacten binnen de Europese Neuroradiologische gemeenschap zou dit onderzoek niet de bekendheid hebben genoten die het tot op heden heeft gehad. Ik waardeer het zeer dat $\mathrm{u}$ mij deze kans heeft gegeven.

Then I would like to express my gratitude to the three German colleagues: professor Armin Thron, doctor Michael Mull, and doctor Timo Krings. After our first meeting in Maastricht and the first two patients on that Friday afternoon in December 2002 a very fruitful collaboration started. Your expertise and the offer to validate the MR angiography technique at your referral centre for vascular abnormalities of the spinal cord substantially improved the scientific value of this thesis. For this I would like to thank you all and I would like to conclude by saying that I feel privileged to have had the opportunity to be acquainted and worked closely together with you all. 
Fons Kessels, gedurende deze promotie hebben wij veel van elkaar kunnen leren. Jij hebt mij statistische kennis bij gebracht op een soms voor mij onnavolgbare wijze en ik jou enkele beginselen van het golfspel. De vrijdagavonden bij Canadian Corner met de inmiddels beruchte pizza taco heb ik zeer gewaardeerd. Bedankt voor je hulp en je gezelschap.

Prof. dr. Werner Mess, de door uw afdeling geregistreerde intra-operatieve electrofysiologie van het ruggenmerg was van onschatbare waarde voor het onderzoek. Hierdoor ontstond de mogelijkheid de klinische toepassing van preoperatieve beeldvorming van de bloedvoorziening van het ruggenmerg te onderzoeken. Bedankt en hopelijk kunnen we in de toekomst blijven samenwerken.

Dr. Geert-Willem Schurink, jouw kritische blik op het klinisch belang van het visualiseren van de Adamkiewicz arterie heeft ertoe geleid dat mijn toewijding, om iedereen te overtuigen dat visualisatie wel degelijk van belang kan zijn, alleen maar verder is gegroeid. Naast deze stimulans wil ik je bedanken voor de aangename samenwerking.

Dr. Tim Leiner, dr. Boudewijn Vasbinder en dr. Vincent Cappendijk. Eindelijk kan ik mij scharen in jullie rijtje van 'doctores'. Bedankt voor al jullie adviezen, interesse en gezelligheid tijdens nationale en internationale "meetings".

Voor het onderzoek is de afdeling Radiologie van groot belang geweest en daarom wil ik de gehele afdeling Radiologie met al zijn medewerkers bedanken. Velen van jullie hebben mij geholpen bij het uitvoeren van mijn onderzoek. De collega promovendi en klinisch fysici wil ik bedanken voor een grandioze tijd. Jaap, ik denk dat iedereen kamergenoot van ons wil worden, want tegelijk begonnen en tegelijk geëindigd, hoog rendement gegarandeerd zou ik zo zeggen. Max, bedankt voor de "ontspannende momenten" waarin wij onze frustraties even konden ventileren. Hoofdlaboranten Henk Schoenmakers en Geert Wijnhoven en jullie teams wil ik buitengewoon bedanken want zonder jullie flexibiliteit zouden al mijn lastminute in plan acties niet zijn vervuld. Henk en Geert ontzettend bedankt voor jullie begrip. Het angio-team bedankt voor jullie interesse en hulp bij het uitvoeren van mijn onderzoek. Ine Kengen bedankt voor je hulp bij het 'boekje'. Als laatste wil ik graag de onmisbare dames van het secretariaat bedanken: Astrid, Brigitte, Christianne, Elfie en Monique.

Chirugie afdeling $\mathrm{B} 4$, bedankt dat jullie ervoor zorgden dat ik de patiënten altijd kon meenemen op de meest onmogelijke momenten. Stella heel veel succes met het afronden van je opleiding en ik kijk uit naar onze toekomstige samenwerking. Iemand die zeker niet mag ontbreken in mijn dankwoord is Claire Meertens. Altijd had je tijd voor mij en kon je een gaatje in de agenda van de baas vinden, ook al was het gaatje er niet. Bedankt voor alles.

Rogier Trompert, bedankt voor de verhelderende anatomische tekeningen die je hebt gemaakt voor dit proefschrift.

Paranimfen Nils Planken en Robert van Boesschoten. Nils inmiddels dr. Planken, ik vond het een eer paranimf te mogen zijn bij jouw promotie. Ik hoop dat je net zoveel van deze dag zult genieten als ik heb gedaan tijdens jouw promotie. Robert beter bekend als Boes, tja wat moet ik zeggen. We hebben allebei al veel meegemaakt in ons korte leventje. Iets wat de vriendschap waarschijn- 
lijk alleen maar hechter heeft gemaakt. Onze vriendschap koester ik zeer. Het betekent veel voor mij dat je een van mijn paranimfen wilt zijn en ik hoop dat we snel weer een weekje kunnen doorbrengen samen met 'Turkem el Baseair'.

Xavier en Fred, jongens het begint wel een beetje saai te worden hoor, alledrie chirurg in opleiding en bijna alledrie gepromoveerd. Eenheidsworsten zullen sommige mensen misschien zeggen, gelukkig weten wij wel beter. I $\mathrm{k}$ ben blij dat ik jullie heb leren kennen en hoop dat we nog lang vrienden zullen blijven.

Lieve Tien, het afgelopen half jaar was 'heavy' jij een nieuwe baan, ik een nieuwe baan en tussendoor moest ik 'nog even' de promotie afmaken. Daarom wil ik je bedanken voor je geduld en begrip. Ik waardeer jouw spontaniteit, waardoor je bijna alles aanpakt en nergens voor terug schrikt, afgezien van vliegtuigen dan.

Pap en mam, bij de generale repetitie in Genève waren jullie er al bij. Vandaag is dan eindelijk de dag waar we met ons drieën naar toe hebben gewerkt. Bedankt voor jullie luisterend oor, onvoorwaardelijke hulp en altijd nuttige adviezen. Ik kan mij geen betere ouders wensen. Ik hoop dat jullie net zoveel zullen genieten van deze dag als ik. 
About the author

List of publications related to this thesis 
Robbert J. Nijenhuis was born on the 28th of February, 1979 in Utrecht, The Netherlands. $\mathrm{He}$ attended high-school (Gymnasium) at College 'de Klop' in Utrecht, and graduated in 1997. Hereafter, he started to study Medicine at maastricht university and participated in research alongside his medical studies at the departments of Medical Education (1999-2000) and Radiology (1999-2002). Apart from performing research he was treasurer of the 4th Maastricht Medical Students Research Conference (2000) and chairman of the association of medical interns (2001). In May 2002 he began with his MD-PhD project, in which he combined two years of internships with scientific research. He obtained his medical doctor degree in September 2004. The research described in this $\mathrm{PhD}$ thesis was performed at the departments of Radiology and Surgery in Maastricht (The Netherlands) and the department of Neuroradiology at the Aachen University Hospital (Germany). For part of this work he was nominated for the Annual Prize of the Dutch Radiological Society (2005). In 2006 he was awarded for giving the best oral presentation at the annual conference of the European Society of Neuroradiology. In January 2007 the author started his surgical residency for a training period of 6 years at the 'Maasland Hospital' in Sittard and the Maastricht University Hospital. 
1. Backes $W H$, Nijenhuis RJ. Inlet arteries or outlet veins of the spinal cord? AJR Am J Roentgenol 2007 (in press).

2. Mull M, Nijenhuis RJ, Backes WH, Krings T, Wilmink JT, Thron A. Value and limitations of contrastenhanced MR Angiography in spinal arteriovenous malformations and dural arteriovenous fistulas. AJNR Am J Ncuroradiol 2007 (in press).

3. Jaspers K, Nijenhuis RJ, Backes WH. Differentiation of spinal cord arteries and veins by MR Angiography. J Magn Reson Imaging 2007 (in press).

4. Krings $\mathrm{T}$, Lasjaunias PL, Franz JH, Mull M, Nijenhuis RJ, Alvarez H, Backes WH, Meinges MHT, Rodesch G, Gilsbach JM, Thron AK. Imaging in spinal vascular disease. Neuroimaging Clin N Am 2007;17:57-72.

5. Nijenhuis RJ, Jacobs MJ, Jaspers K, Reijnders M, van Engelshoven JM, Leiner T, Backes WH. Comparison of computed tomographic angiography and magnetic resonance angiography for the localization of the Adamkiewicz artery. J Vasc Surg 2007;45:677-85.

6. Jacobs MJ, Mommertz G, Koeppel TA, Langer S, Nijenhuis RJ, Mess WH, Schurink GW.

Surgical repair of thoracoabdominal aortic aneurysms. J Cardiovasc Surg 2007;48:49-58.

7. Schurink GW, Nijenhuis RJ, Backes WH, Mess WH, de Haan MW, Mochtar B, Jacobs MJ. Spinal cord circulation and function monitoring in endovascular treatment of thoracic descending aortic aneurysms. Ann Thorac Surg 2007;83:S877-81; discussion S890-2.

8. Nijenhuis RJ, Jacobs MJ, Mess WH, van Engelshoven JMA, Backes WH. MR Angiography and neuromonitoring to assess spinal cord perfusion in aortic surgery. J Vasc Surg 2007;45:71-7; discussion 77-8.
9. Nijenhuis RJ, Mull M, Thron AK, Wilmink JT, Backes WH. MR Angiography of the Adamkiewicz artery validated by digital subtraction angiography. AJNR Am J Neuroradiol 2006;27:1565-72.

10. Nijenhuis RJ, Jacobs MJ, van Engelshoven JMA, Backes WH. Magnetic resonance angiography of the Adamkiewicz artery and great medullary vein: Postmortem validation. AJNR Am I Neuroradiol 2006;27:1573-5.

11. Jacobs MJ, Mess WH, Mochtar B, Nijenhuis RJ, Statius van Eps RG, Schurink GW. The value of motor evoked potential in reducing paraplegia during thoracoabdominal repair. J Vasc Surg 2006;43:239-46.

12. Nijenhuis RJ, Gerretsen $S$, Leiner $T$, Jacobs $M J$, van Engelshoven JM, Backes WH. Comparison of $0.5-\mathrm{M}$ Gd-DTPA with 1.0-M gadobutrol for magnetic resonance angiography of the supplying arteries of the spinal cord in thoracoabdominal aortic aneurysm patients. J Magn Reson Imaging 2005;22:136-144.

13. Nijenhuis RJ, Leiner T, Cornips E, Wilmink J, Jacobs MJ, van Engelshoven J, Backes WH. Spinal cord feeding arteries at MR angiography for thoracoscopic spinal surgery: feasibility study and implications for surgical approach. Radiology 2004;233:541-547.

14. Backes WH, Nijenhuis RJ, Mull $M$, Thron AK, Wilmink JT. Contrast-enhanced MR angiography of the spinal arteries: current possibilities and limitations. Rivista di Neuroradiologin 2004;17:282-291. 